quatrième série-tome $44 \quad$ fascicule 2 mars-avril 2011

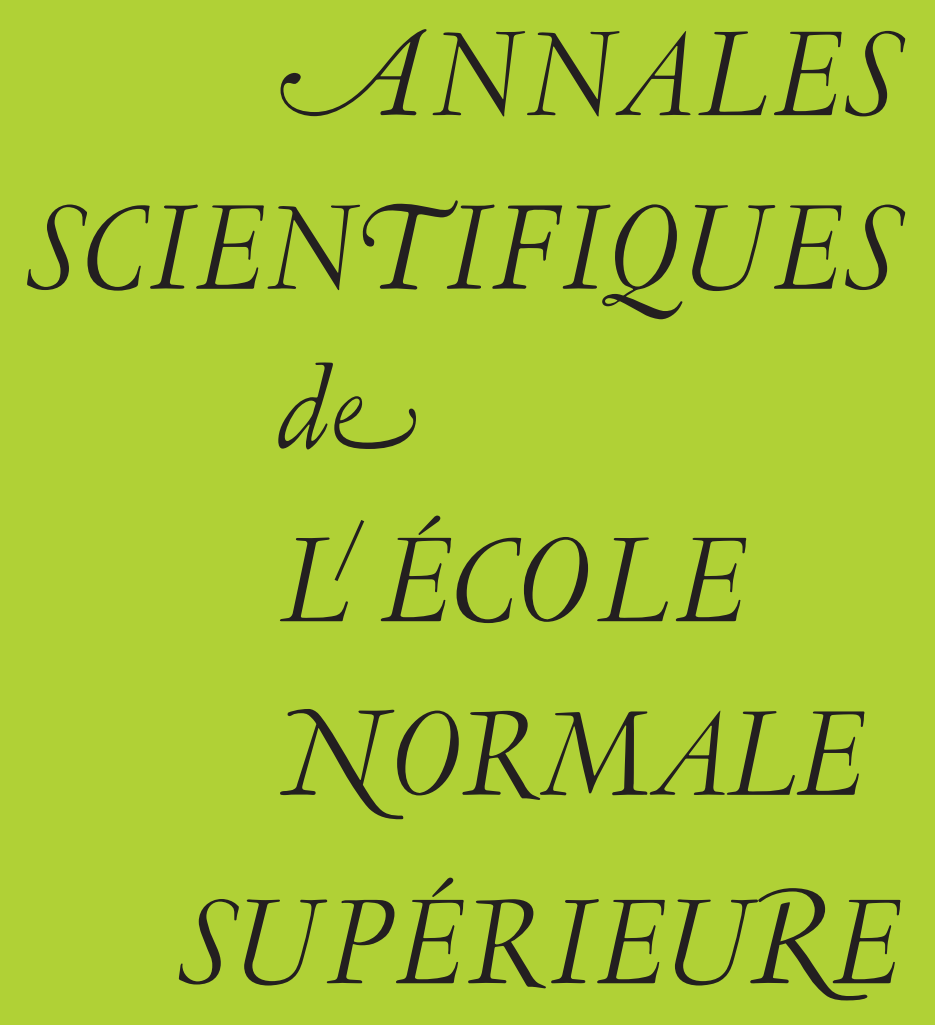

Alain GENESTIER \& Vincent LAFFORGUE

Théorie de Fontaine en égales caractéristiques 
Ann. Scient. Éc. Norm. Sup.

$4^{\text {e }}$ série, t. 44, 2011, p. 263 à 360

\title{
THÉORIE DE FONTAINE EN ÉGALES CARACTÉRISTIQUES
}

\author{
Par Alain GENESTiER et Vincent LAFForgUE
}

RÉsumÉ. - Les chtoucas locaux sont des analogues en égales caractéristiques des groupes $p$-divisibles - par exemple on leur associe un module de Tate, qui est un module libre sur l'anneau d'entiers d'un corps local $K$ de caractéristique positive. Nous associons à un chtouca local une structure de Hodge (ou, plus précisément, une structure de Hodge-Pink), ce qui induit un morphisme de périodes analogue à celui construit par Rapoport et Zink. Pour les structures de Hodge-Pink définies sur une extension finie de $K$ nous démontrons un analogue du théorème " faiblement admissible implique admissible» de Colmez et Fontaine. Nous développons aussi une théorie entière. Les démonstrations sont élémentaires et ne font pas intervenir de clôture algébrique de $K$. Les arguments utilisés dans la théorie entière sont très proches de ceux qui interviennent dans la théorie rationnelle.

Abstract. - Local shtukas are analogs in equal characteristics of $p$-divisible groups: for example one can associate to them a Tate module, which is a free module over the ring of integers of a local field $K$ of positive characteristic. We associate to a local shtuka a Hodge structure (or more precisely a Hodge-Pink structure) which gives rise to a period morphism analogous to the one constructed by Rapoport and Zink. For Hodge-Pink structures defined over a finite extension of $K$ we prove an analog of the "weakly admissible implies admissible" theorem of Colmez and Fontaine. We also develop an integral theory. The proofs are elementary and do not use an algebraic closure of $K$. The arguments used in the integral theory are very close to those used in the rational theory.

Soient $K$ un corps local non archimédien de caractéristique $p$, de corps résiduel $\mathbb{F}_{q}$, et $\theta$ son anneau d'entiers. On choisit une fois pour toutes une uniformisante $\pi$ de $\theta$, ce qui identifie $\theta$ à $\mathbb{F}_{q}[[\pi]]$ et $K$ à $\mathbb{F}_{q}((\pi))$.

Soient $S$ un schéma formel adique sur $\operatorname{Spf} \theta\left(\theta_{S}\right.$ est donc complet pour une topologie adique relativement à un faisceau d'idéaux qui contient $\pi$; pour nous complet sous-entendra toujours séparé). Soit $\oslash \widehat{\otimes} \vartheta_{S}$ le faisceau sur $S$ qui associe à un ouvert affine $\operatorname{Spf} B$ le complété $\emptyset \widehat{\otimes} B$ de $\Theta \otimes_{\mathbb{F}_{q}} B$ vis-à-vis de la topologie $\pi \otimes 1$-adique. On notera toujours $z$ au lieu de $\pi \otimes 1$, en gardant la notation $\pi$ pour $1 \otimes \pi$ ( $z$ et $\pi$ sont donc deux sections partout définies du faisceau $\theta \widehat{\otimes} \theta_{S}$ ). L'isomorphisme $\theta=\mathbb{F}_{q}[[\pi]]$ induit par le choix de l'uniformisante $\pi$ fournit une identification $\theta \widehat{\otimes} B=B[[z]]$. 
Soit Fr l'endomorphisme de $\theta_{S}$ qui agit par $b \mapsto b^{q}$ sur les sections locales de $\theta_{S}$. Pour tout faisceau $M$ de $\theta \widehat{\otimes} \theta_{S}$-modules, on note

$$
{ }^{\tau} M=M \otimes_{\partial \otimes \theta_{S}, 1 \widehat{\otimes} \mathrm{Fr}} \theta \widehat{\otimes} \theta_{S} .
$$

Si $x$ est une section de $M$, on note ${ }^{\tau} x$ la section $x \otimes 1$ de $^{\tau} M$.

DÉfinition 0.1. - Un chtouca local de rang $r$ sur $S$ est une paire $\left(M, \phi_{M}\right)$ formée d'un faisceau $M$ de $\theta \widehat{\otimes} \theta_{S}$-modules qui est localement ${ }^{(1)}$ libre de rang $r$ pour la topologie de Zariski sur $S$, et d'un isomorphisme

$$
\phi_{M}:{ }^{\tau} M\left[\frac{1}{z-\pi}\right] \rightarrow M\left[\frac{1}{z-\pi}\right]
$$

tel que, pour certains entiers $s, t \in \mathbb{Z}$, on ait

$$
(z-\pi)^{t} M \subset \phi_{M}\left({ }^{\tau} M\right) \subset(z-\pi)^{s} M .
$$

Dans ce cas on dira que $M=\left(M, \phi_{M}\right)$ est d'amplitude $\subset[s, t]$.

Remarquons que la condition $(z-\pi)^{t} M \subset \phi_{M}\left({ }^{\tau} M\right)$ équivaut à $\phi_{M}^{-1}(M) \subset(z-\pi)^{-t \tau} M$. Les chtoucas locaux d'amplitude $\subset[s, t]$ forment un champ pour la topologie de Zariski.

Un morphisme de chtoucas locaux $\left(M, \phi_{M}\right) \rightarrow\left(M^{\prime}, \phi_{M^{\prime}}\right)$ sur $S$ est un morphisme de faisceaux de $\theta \widehat{\otimes} \theta_{S}$-modules $f: M \rightarrow M^{\prime}$, tel que $\phi_{M^{\prime}} \circ{ }^{\tau} f=f \circ \phi_{M}$. La catégorie des chtoucas locaux sur $S$ est $\theta$-linéaire.

Une isogénie est un morphisme $f:\left(M, \phi_{M}\right) \rightarrow\left(M^{\prime}, \phi_{M^{\prime}}\right)$ tel qu'il existe un morphisme $g:\left(M^{\prime}, \phi_{M^{\prime}}\right) \rightarrow\left(M, \phi_{M}\right)$ et une fonction localement constante $e: S \rightarrow \mathbb{N}$ pour lesquels $g \circ f=z^{e}$ et $f \circ g=z^{e}$. La catégorie des chtoucas locaux à isogénie près s'obtient à partir de la catégorie des chtoucas locaux en inversant les isogénies. On emploiera aussi le terme d'isochtouca local comme synonyme de chtouca local à isogénie près.

Lorsque $\left(M, \phi_{M}\right)$ est un chtouca local sur $S$ et $S^{\prime} \rightarrow S$ est un morphisme de Spf $\theta$-schémas formels, le changement de base $\left(M, \phi_{M}\right)_{S^{\prime}}=\left(M \otimes_{\vartheta \widehat{\otimes} \vartheta_{S}} \oslash \widehat{\otimes} \vartheta_{S^{\prime}}, \phi_{M} \otimes 1\right)$ est un chtouca local sur $S^{\prime}$.

On dit qu'un chtouca local est minuscule s'il est d'amplitude $\subset[0,1]$. Plus concrètement on a la définition suivante.

Définition 0.2. - Un chtouca local minuscule de rang $r$ sur $S$ est une paire $\left(M, \phi_{M}\right)$ formée d'un faisceau $M$ de $\Theta \widehat{\otimes} \Theta_{S}$-modules qui est localement libre de rang $r$ pour la topologie de Zariski sur $S$, et d'un morphisme $\phi_{M}:{ }^{\tau} M \rightarrow M$ tel qu'il existe $\psi: M \rightarrow{ }^{\tau} M$ vérifiant $\phi_{M} \circ \psi=(z-\pi)$ et $\psi \circ \phi_{M}=(z-\pi)$.

(1) Dans le cas particulier où $S$ est un schéma affine $\operatorname{Spec} B$ (muni de la topologie triviale) le $\theta \widehat{\otimes} B$-module $M$ des sections globales n'est pas nécessairement localement libre, contrairement à ce qui est affirmé dans [26] (en effet pour $f \in B$ l'inclusion $(\theta \widehat{\otimes} B)\left[(1 \otimes f)^{-1}\right] \subset \vartheta \widehat{\otimes}\left(B\left[f^{-1}\right]\right)$ est en général stricte). Cette confusion y est cependant sans conséquence : il suffit d'y remplacer systématiquement l'expression «localement libre pour la topologie de Zariski $\operatorname{sur} \operatorname{Spec} \theta \widehat{\otimes} B »$ par «localement libre pour la topologie de Zariski sur Spf $\oslash \widehat{\otimes} B »$. 
Dans la situation de la définition précédente $\psi$ est unique et sera noté $\psi_{M}$. D'autre part $\phi_{M}$ et $\psi_{M}$ sont injectifs. D'après le a) du lemme 1.1, $\psi_{M}(M) /(z-\pi)^{\tau} M$ est un sous- $\theta_{S}$-module localement facteur direct du $\theta_{S}$-module localement libre de type fini ${ }^{\tau} M /(z-\pi)^{\tau} M$, et on verra que l'inclusion

$$
\psi_{M}(M) /(z-\pi)^{\tau} M \subset{ }^{\tau} M /(z-\pi)^{\tau} M
$$

joue le rôle de la filtration de Hodge (cf. [2] et le paragraphe I.3 de [26]).

On définit un morphisme (resp. une isogénie) de chtoucas locaux minuscules comme un morphisme (resp. une isogénie) de chtoucas locaux.

La catégorie des chtoucas locaux minuscules est une sous-catégorie pleine de la catégorie des chtoucas locaux et elle est donc aussi $\theta$-linéaire.

Les chtoucas locaux minuscules fournissent l'analogue en égales caractéristiques des groupes $p$-divisibles. Nous allons rappeler comment associer un «module $\theta$-divisible» à un chtouca local minuscule, en commençant par le résultat général suivant.

Proposition 0.3 (Drinfeld [19] paragraphe 2, SGA $3 V I I_{A}$ 7.4, [26] chapitre 1)

Soient $S$ un schéma sur $\mathbb{F}_{q}$ et $r \in \mathbb{N}$. On note $\sigma$ le morphisme de Frobenius de $S$ dans lui-même qui à une section locale $x$ du faisceau structural associe $x^{q}$. Alors il $y$ a une anti-équivalence de catégories entre

- les $\theta_{S}$-modules localement libres $M$ de rang $r$ munis d'un morphisme $\phi_{M}: \sigma^{*}(M) \rightarrow M$,

- les schémas en groupe finis et plats $G$ sur $S$, de présentation finie, d'ordre $q^{r}$, munis d'une action de $\mathbb{F}_{q}$ qui induit sur le cotangent de $G$ l'action de $\mathbb{F}_{q}$ provenant du fait que $S$ est un schéma sur $\mathbb{F}_{q}$, et dont le Verschiebung est nul.

Rappelons, d'après Drinfeld, le foncteur de la première catégorie dans la seconde. À $\left(M, \phi_{M}\right)$ il associe le schéma en groupes sur $S$ avec action de $\mathbb{F}_{q}$ noté $\operatorname{Gr}\left(M, \phi_{M}\right)$ et défini de la manière suivante : si on note encore $M^{*}$ et $\sigma^{*}\left(M^{*}\right)$ les schémas en groupes qui sont les espaces totaux de $M^{*}$ et $\sigma^{*}\left(M^{*}\right)=\sigma^{*}(M)^{*}$, le transposé de $\phi_{M}$ et le Frobenius relatif $\sigma_{M^{*} / S}$ de $M^{*}$ sur $S$ sont des morphismes de schémas en groupes de $M^{*}$ vers $\sigma^{*}\left(M^{*}\right)$ et on pose alors

$$
\operatorname{Gr}\left(M, \phi_{M}\right)=\operatorname{Ker}\left({ }^{t} \phi_{M}-\sigma_{M^{*} / S}: M^{*} \rightarrow \sigma^{*}\left(M^{*}\right)\right) .
$$

Drinfeld montre en complément que $\operatorname{Lie}^{*}\left(\operatorname{Gr}\left(M, \phi_{M}\right)\right)=\operatorname{Coker}\left(\phi_{M}\right)$, où Lie* $\left(\operatorname{Gr}\left(M, \phi_{M}\right)\right)$ désigne l'image inverse de $\Omega_{\operatorname{Gr}\left(M, \phi_{M}\right) / S}^{1}$ par la section nulle. Drinfeld construit un quasiinverse explicite : à $G$ on associe le faisceau $M$ des homomorphismes de schémas en groupes commutatifs avec action de $\mathbb{F}_{q}$ de $G$ dans $\mathbb{G}_{a}$ (muni d'une action de $\theta_{S}$ qui vient de l'action de $\theta_{S}$ sur $\mathbb{G}_{a}$ par homothéties, et d'un morphisme $\sigma^{*}(M) \rightarrow M$ qui vient du morphisme de Frobenius $x \mapsto x^{q}$ de $\mathbb{G}_{a}$ dans lui-même au-dessus de $S$ ).

Soit maintenant $\left(M, \phi_{M}\right)$ un chtouca local minuscule sur $S$. Pour tout entier $n \in \mathbb{N}^{*}$ on pose $G_{n}=\operatorname{Gr}\left(M / z^{n} M, \phi_{M} \bmod z^{n}\right)$. Alors $\left(G_{n}\right)$ est une suite de schémas en groupe finis et plats de présentation finie, munis d'une action de $\theta$, avec des inclusions $G_{n} \rightarrow G_{n+m}$, donnant lieu aux suites exactes habituelles. L'existence de $\psi$ dans la définition 0.2 assure que l'action de $\theta$ sur $\operatorname{Lie}^{*}\left(G_{n}\right)$ est celle provenant du fait que $S$ est un schéma formel sur Spf $\theta$.

Passons maintenant en revue le contenu de cet article.

Le premier paragraphe est consacré à des préliminaires. Dans les paragraphes 2 et 3 nous étudions les chtoucas locaux à isogénie près sur une base quelconque. Nous introduisons la 
notion de pseudo-isochtouca local, qui est équivalente à celle de chtouca local à isogénie près (aussi appelé isochtouca) lorsque la base est le spectre d'un anneau de valuation réelle complet dans lequel l'image de $\pi$ est non nulle et de valuation $>0$. Si la base est affine, $\pi$-adique et sans $\pi$-torsion, nous associons aux pseudo-isochtoucas locaux rigidifiés des structures de Hodge-Pink sur sa fibre générique au sens de Raynaud (ces structures ont été introduites par Pink dans le cadre de l'uniformisation des t-motifs, voir [49]). Dans le cas minuscule une structure de Hodge-Pink est simplement une structure de Hodge d'amplitude $\subset[0,1]$ alors qu'en général elle induit seulement (sur les strates d'une stratification) une structure de Hodge qui ne suffit pas en retour à la déterminer. Lorsque la base est un schéma formel adique localement de type fini (au sens du paragraphe 0.2 de [5]) on associe aux pseudoisochtoucas locaux rigidifiés des structures de Hodge-Pink sur la fibre générique au sens de Raynaud-Berthelot de ce schéma formel : on peut recouvrir cette variété rigide analytique par des spectres d'algèbres de Tate, dont les anneaux d'entiers sont des algèbres $\pi$-adiques et sans $\pi$-torsion, de sorte qu'on est en fait ramené au cas affine $\pi$-adique et sans $\pi$-torsion et on peut supposer de plus que les modules considérés sont libres. Dans le cas universel où la base est un espace de modules de chtoucas locaux rigidifiés, la structure de Hodge-Pink associée au chtouca local universel définit une application de la fibre générique au sens de Raynaud-Berthelot vers l'espace de modules des structures de Hodge-Pink, appelée application des périodes (voir [51] chapitre 4 et [31]).

Dans le paragraphe 4 nous cherchons quelles puissances divisées sont adaptées pour une théorie entière sur une base $\pi$-adique et sans $\pi$-torsion. La réponse n'est satisfaisante que dans le cas minuscule; dans les paragraphes 5 et 6 nous développons une telle théorie entière pour les chtoucas locaux minuscules sur une base $\pi$-adique. Le paragraphe 5 est consacré à la théorie de Dieudonné cristalline des chtoucas minuscules. Nous y associons un «cristal de Honda-Gross-Hopkins » à tout chtouca minuscule. Ces cristaux de Honda-Gross-Hopkins sont définis en utilisant une notion de puissances divisées due à Honda et Gross-Hopkins, moins contraignante que la notion plus habituelle due à Grothendieck et Berthelot. La construction originale de Gross-Hopkins [33], utilisant la notion de quasi-logarithme, ne permet de traiter que le cas où la base est sans $\pi$-torsion. L'analogue en égales caractéristiques de la construction de Messing [48] nécessite quant à elle des puissances divisées nilpotentes au sens de Grothendieck et Berthelot. Notre construction lève donc ces deux limitations. À la fin du paragraphe 5 nous la comparons à celle de Messing dans le cas particulier d'un épaississement à puissances divisées nilpotentes au sens de Grothendieck et Berthelot (nous ne l'avons pas comparée à celle de Mazur-Messing [47] qui évite la condition de nilpotence). Dans le paragraphe 6 nous démontrons un analogue du théorème de relèvement de Grothendieck et Messing pour les épaississements à puissances divisées nilpotentes au sens de Grothendieck et Berthelot.

Les paragraphes 7, 8, 10 et 11 sont consacrés au cas où la base est le spectre d'un anneau de valuation discrète complet dans lequel l'image de $\pi$ est non nulle et de valuation $>0$, et dont le corps résiduel est parfait. Les paragraphes 7 et 8 montrent que sous cette hypothèse la catégorie des chtoucas locaux à isogénie près est équivalente à celle des isochtoucas sur le corps résiduel munis d'une structure de Hodge-Pink faiblement admissible. Ce résultat est un analogue en égales caractéristiques du théorème "faiblement admissible implique admissible » de Colmez et Fontaine [16]. Hartl [31] a étendu ce résultat à certains anneaux 
de valuation réelle non nécessairement discrète en adaptant en égales caractéristiques les démonstrations du théorème «faiblement admissible implique admissible » dues à Berger [3] et Kisin [39], qui reposent sur les résultats de Kedlaya [38]. Hartl a donc besoin de [32] et du premier paragraphe de [31] où sont établis en égales caractéristiques des résultats analogues à ceux de Kedlaya. Notre démonstration est beaucoup plus élémentaire. Le paragraphe 9 donne un contre-exemple pour un anneau de valuation rationnelle non discrète. Ce contreexemple a aussi été considéré par Hartl dans [29] mais nous le voyons sous un angle différent.

Enfin dans le paragraphe 10 nous commençons pour les chtoucas locaux minuscules une théorie de Hodge entière dans l'esprit de [14, 20,55] (notre approche a ceci de commun avec celle de Zink que nous n'introduisons pas de clôture algébrique du corps des fractions). Pour les chtoucas locaux non minuscules le paragraphe 4 montre qu'une théorie entière est problématique. Cependant dans le paragraphe 11 nous obtenons des résultats analogues à ceux de $[14,15,39,40,45]$ pour des chtoucas locaux non nécessairement minuscules, mais vérifiant une condition que nous appelons « tranquillité » et qui rappelle la transversalité de Griffiths. Les chtoucas locaux minuscules sont toujours tranquilles. La condition de tranquillité prend plusieurs formes, dont certaines gardent un sens en inégales caractéristiques, et elles y sont toujours vérifiées.

Dans tout cet article nous ne considérons que la situation de bonne réduction. Plus précisément dans les paragraphes 7,8,10 et 11 les chtoucas locaux sont définis sur un anneau de valuation discrète et non simplement sur son corps des fractions. Quelques réflexions sur le cas de mauvaise réduction nous ont convaincus que la situation en égales caractéristiques était très différente de celle qu'on rencontre en inégales caractéristiques (voir [18, 25, 42, 43]).

Le point de vue adopté dans cet article est de n'introduire aucun anneau analogue aux anneaux de Fontaine (de tels analogues sont décrits en détail dans [30]), et en particulier, si la base est le spectre d'un anneau de valuation discrète, de ne pas recourir à une clôture algébrique de son corps des fractions. Ce point de vue est mieux adapté à la théorie entière : le lecteur pourra constater que la preuve du théorème 7.3 « faiblement admissible implique admissible », présentée dans le paragraphe 8 , est très voisine de la preuve des théorèmes 10.3 et 11.9, qui sont nos résultats principaux en théorie entière. Ce point de vue permet aussi de considérer des bases très générales : la proposition 3.12 permet sur une base $\pi$-adique et sans $\pi$-torsion quelconque de retrouver un pseudo-isochtouca local rigidifié modulo $\pi$ à partir de sa structure de Hodge-Pink. En revanche la méthode de Hartl (qui utilise des anneaux analogues aux anneaux de Fontaine) montre le théorème «faiblement admissible implique admissible » pour tous les anneaux de valuation réelle complets tels que pour aucun $\alpha \in \mathbb{R}_{+}^{*}$ l'image de la valuation ne contienne tous les $\alpha p^{-n}$ avec $n \in \mathbb{N}$ (voir le théorème 2.5.3 de [31] pour la condition précise), alors que notre démonstration ne marche que pour les anneaux de valuation discrète.

Le lecteur intéressé seulement par la théorie rationnelle dans le cas des anneaux de valuation discrète peut lire directement les paragraphes 7 et 8 .

Nous remercions Laurent Berger, Christophe Breuil, Olivier Brinon, Xavier Caruso, Pierre Colmez, Vladimir Drinfeld, Laurent Fargues, Urs Hartl, Mark Kisin et Richard Pink pour des discussions très utiles, et le rapporteur pour sa lecture attentive et ses remarques. 


\section{Résultats préliminaires}

Dans cet article tout anneau commutatif sera supposé unitaire et libre signifiera toujours libre de type fini. Soit $A$ un anneau commutatif. On note $A[[x]]$ l'anneau des séries formelles et $A((x))=A[[x]]\left[\frac{1}{x}\right]$ l'anneau des séries de Laurent à coefficients dans $A$.

Le a) du lemme suivant a servi dans l'introduction.

Lemme 1.1. - Soit $r \in \mathbb{N}^{*}$ un entier strictement positif.

a) Soit $g \in G L_{r}(A((x)))$. Soient $s, t \in \mathbb{Z}$ tels que g appartienne à $x^{s} M_{r}(A[[x]])$ et $g^{-1}$ appartienne à $x^{-t} M_{r}(A[[x]])$. Alors $x^{t} A[[x]]^{r} \subset g\left(A[[x]]^{r}\right) \subset x^{s} A[[x]]^{r}$ et $g\left(A[[x]]^{r}\right) / x^{t} A[[x]]^{r}$ est un sous-A-module facteur direct du A-module libre $x^{s} A[[x]]^{r} / x^{t} A[[x]]^{r}$.

b) Soient $s, t \in \mathbb{Z}$ et $M$ un $A[[x]]$-module vérifiant $x^{t} A[[x]]^{r} \subset M \subset x^{s} A[[x]]^{r}$ et tel que $M / x^{t} A[[x]]^{r}$ soit un sous-A-module facteur direct dans $x^{s} A[[x]]^{r} / x^{t} A[[x]]^{r}$. Alors, localement pour la topologie de Zariski de $A, M$ est un $A[[x]]$-module libre de rang $r$ et il existe $g \in G L_{r}(A((x)))$ tel que $M=g\left(A[[x]]^{r}\right)$.

Ce lemme est bien connu (voir par exemple les propositions 3.1 et 3.2.1 de [35]). Nous en donnons une démonstration pour la commodité du lecteur.

Démonstration. - a) Choisissons un idempotent

$$
p \in \operatorname{End}_{A}\left(x^{s-t} A[[x]]^{r} / x^{t-s} A[[x]]^{r}\right)
$$

dont l'image soit $A[[x]]^{r} / x^{t-s} A[[x]]^{r}$ : par exemple l'oubli des puissances strictement négatives dans un développement en puissances de $x$. Alors $g p g^{-1}$ est un idempotent de $\operatorname{End}_{A}\left(g\left(x^{s-t} A[[x]]^{r}\right) / g\left(x^{t-s} A[[x]]^{r}\right)\right)$ dont l'image est $g\left(A[[x]]^{r}\right) / g\left(x^{t-s} A[[x]]^{r}\right)$. Or on a

$$
g\left(x^{t-s} A[[x]]^{r}\right) \subset x^{t} A[[x]]^{r} \subset g\left(A[[x]]^{r}\right) \subset x^{s} A[[x]]^{r} \subset g\left(x^{s-t} A[[x]]^{r}\right)
$$

et $g p g^{-1}$ induit donc un idempotent de $\operatorname{End}_{A}\left(x^{s} A[[x]]^{r} / x^{t} A[[x]]^{r}\right)$ dont l'image est $g\left(A[[x]]^{r}\right) / x^{t} A[[x]]^{r}$.

b) Localement pour la topologie de Zariski de $A$, il existe un sous- $A$-module libre $Q \subset x^{s-1} A[[x]]^{r} / x^{t} A[[x]]^{r}$ supplémentaire de $M / x^{t} A[[x]]^{r}$. L'intersection de $x Q$ et de $\left(M / x^{t+1} A[[x]]^{r}\right)$ dans $x^{s} A[[x]]^{r} / x^{t+1} A[[x]]^{r}$ est un $A$-module projectif de rang $r$, supplémentaire de $x M$ dans $M$, donc localement $M / x M$ est un $A$-module libre de rang $r$ et $M$ est un $A[[x]]$-module libre de rang $r$.

Le a) de ce lemme va nous permettre de montrer l'existence locale de relèvements de chtoucas locaux minuscules. Le résultat analogue pour les groupes $p$-divisibles est bien connu [34].

Lemme 1.2. - Soient B une Q-algèbre complète pour la topologie $\pi$-adique et I un idéal fermé de $B$ formé d'éléments topologiquement nilpotents. Alors tout chtouca local minuscule $\left(M, \phi_{M}\right)$ sur $B / I$ se relève, localement pour la topologie de Zariski de $\operatorname{Spf}(B / I)$, en un chtouca local minuscule $\left(\widetilde{M}, \phi_{\widetilde{M}}\right)$ sur $B$. 
Démonstration. - Localement on peut supposer que $M$ est libre sur $\theta \widehat{\otimes}(B / I)$ et on choisit une base de $M$. Grâce au a) du lemme 1.1, localement on peut supposer que $(z-\pi) \phi_{M}^{-1}(M) /(z-\pi)^{\tau} M$ est un sous- $B / I$-module libre de ${ }^{\tau} M /(z-\pi)^{\tau} M$ et qu'il existe $P, Q \in G L_{r}(\Theta \widehat{\otimes}(B / I))$ et $D \in M_{r}(\Theta \widehat{\otimes}(B / I))$ une matrice diagonale dont les coefficients diagonaux sont égaux à 1 ou $z-\pi$, tels que $\phi_{M}=P D Q$. On relève $P$ et $Q$ en $\widetilde{P}, \widetilde{Q} \in M_{r}(\Theta \widehat{\otimes} B)$ et comme $I$ est formé d'éléments topologiquement nilpotents on a $\widetilde{P}, \widetilde{Q} \in G L_{r}(\theta \widehat{\otimes} B)$. On note $\widetilde{D} \in M_{r}(\Theta \widehat{\otimes} B)$ la matrice égale à $D$ obtenue en considérant $\pi$ comme un élément de $B$ au lieu de $B / I$. Alors $\widetilde{M}=(\emptyset \widehat{\otimes} B)^{r}$ muni de $\phi_{\widetilde{M}}=\widetilde{P} \widetilde{D} \widetilde{Q}$ est un chtouca local minuscule sur $B$ qui relève $\left(M, \phi_{M}\right)$.

\section{La catégorie des pseudo-isochtoucas locaux}

Soit $S$ un schéma formel sur $\operatorname{Spf} \theta$ (complet pour une topologie adique relativement à un faisceau d'idéaux qui contient $\pi$ ). Soit $\theta \widehat{\otimes} \vartheta_{S}\left[\frac{1}{z}\right]$ le faisceau qui sur un ouvert affine $\operatorname{Spf} B$ ( $B$ étant une $\theta$-algèbre complète pour une topologie adique relativement à un idéal qui contient $\pi$ ) vaut $\Theta \widehat{\otimes} B\left[\frac{1}{z}\right]$. L'isomorphisme $\theta=\mathbb{F}_{q}[[\pi]]$ fournit l'égalité $\theta \widehat{\otimes} B\left[\frac{1}{z}\right]=B((z))$.

Définition 2.1. - Soient $s, t \in \mathbb{Z}$. On appelle pseudo-isochtouca local de rang $r$ sur $S$ et d'amplitude $\subset[s, t]$ une paire $\left(N, \phi_{N}\right)$ formée d'un $\widehat{\theta} \widehat{\otimes} \oslash_{S}\left[\frac{1}{z}\right]$-module $N$ localement libre (pour la topologie de Zariski sur $S$ ) de rang $r$, muni d'un isomorphisme de $\Theta \widehat{\otimes} \vartheta_{S}\left[\frac{1}{z}, \frac{1}{z-\pi}\right]$-modules $\phi_{N}:{ }^{\tau} N\left[\frac{1}{z-\pi}\right] \rightarrow N\left[\frac{1}{z-\pi}\right]$ tel que $(z-\pi)^{t} N \subset \phi_{N}\left({ }^{\tau} N\right) \subset(z-\pi)^{s} N$ et que la condition suivante soit satisfaite:

(PIL) localement pour la topologie de Zariski sur $S$, il existe un $\theta \widehat{\otimes} \theta_{S}$-module libre $M$ de rang $r$ muni d'un isomorphisme $M \otimes_{\vartheta \widehat{\otimes} \vartheta_{S}} \oslash \widehat{\otimes} \vartheta_{S}\left[\frac{1}{z}\right]=N$ et une constante $C$, tels que pour tout $n \in \mathbb{N}^{*}, \phi_{N}{ }^{\tau} \phi_{N} \ldots \tau^{n-1} \phi_{N}$ appartienne à

$$
z^{-C}(z-\pi)^{s}\left(z-\pi^{q}\right)^{s} \cdots\left(z-\pi^{q^{n-1}}\right)^{s} \operatorname{Hom}_{\hat{\partial} \widehat{\otimes} \vartheta_{S}}\left(\tau^{n} M, M\right)
$$

et que $\left(\phi_{N}^{\tau} \phi_{N} \ldots \tau^{n-1} \phi_{N}\right)^{-1}$ appartienne à

$$
z^{-C}(z-\pi)^{-t}\left(z-\pi^{q}\right)^{-t} \cdots\left(z-\pi^{q^{n-1}}\right)^{-t} \operatorname{Hom}_{\vartheta \widehat{\otimes} \vartheta_{S}}\left(M,{ }^{\tau^{n}} M\right) .
$$

Remarque. - Dans cette définition la constante $C$ est bornée localement sur $S$ alors que $s$ et $t$ sont constants. Cela est volontaire (on aurait pu définir les chtoucas locaux et les pseudo-isochtoucas locaux en bornant seulement localement l'amplitude mais ce genre d'objet ne paraît pas très naturel).

Un morphisme de pseudo-isochtoucas locaux $\left(N, \phi_{N}\right) \rightarrow\left(N^{\prime}, \phi_{N^{\prime}}\right)$ sur $S$ est un morphisme de faisceaux de $\theta \widehat{\otimes} \theta_{S}\left[\frac{1}{z}\right]$-modules $f: N \rightarrow N^{\prime}$ tel que $\phi_{N^{\prime}} \circ{ }^{\tau} f=f \circ \phi_{N}$. La catégorie des pseudo-isochtoucas locaux sur $S$ est $K$-linéaire.

Pour tout chtouca local $M$ sur $S, M \otimes_{\partial \widehat{\otimes} \theta_{S}} \oslash \widehat{\otimes} \vartheta_{S}\left[\frac{1}{z}\right]$ est un pseudo-isochtouca local sur $S$. On obtient ainsi un foncteur pleinement fidèle de la catégorie des chtoucas locaux à isogénie près sur $S$ dans celle des pseudo-isochtoucas locaux sur $S$.

Remarque. - Si $S$ a de la $\pi$-torsion et $M$ est un chtouca local sur $S$, l'amplitude du pseudo-isochtouca local $M \otimes_{\vartheta \widehat{\otimes} \vartheta_{S}} \oslash \widehat{\otimes} \vartheta_{S}\left[\frac{1}{z}\right]$ (au sens de la définition 2.1) peut être strictement plus petite que celle de $M$ (au sens de la définition 0.1 ). 
Soit $\vartheta_{L}$ un anneau de valuation réelle (non nécessairement discrète) complet. On note $m_{L}$ son idéal maximal. On note $L$ le corps des fractions de $\theta_{L}$. On suppose $\theta_{L}$ muni d'une structure de $\theta$-algèbre de sorte que l'image de $\pi$ dans $\theta_{L}$ soit un élément non nul de $m_{L}$, que l'on note encore $\pi$.

Proposition 2.2. - Le foncteur de la catégorie des chtoucas locaux à isogénie près sur $\theta_{L}$, vers celle des pseudo-isochtoucas locaux sur $\theta_{L}$, est une équivalence de catégories.

Démonstration. - Seule l'essentielle surjectivité reste à démontrer. Soient $s, t \in \mathbb{Z}$. Il s'agit d'établir qu'un pseudo-isochtouca local $N$ d'amplitude $\subset[s, t]$ est associé à un chtouca local $M$ d'amplitude $\subset[s, t]$ (évidemment non unique, mais unique à isogénie près). Par le lemme 2.3 ci-dessous il suffit de trouver un $L[[z]]$-réseau $V$ dans le $L((z))$-espace vectoriel $N \otimes_{\widehat{\vartheta} \widehat{\otimes} \vartheta_{L}\left[\frac{1}{z}\right]} L((z))$, qui soit préservé par $\phi_{N} \otimes_{\widehat{\partial} \widehat{\otimes} \vartheta_{L}\left[\frac{1}{z-\pi}, \frac{1}{z}\right]} \operatorname{Id}_{L((z))}$ (c'est-à-dire $\left.\phi_{N} \otimes_{\partial \widehat{\otimes} \vartheta_{L}\left[\frac{1}{z-\pi}, \frac{1}{z}\right]} \operatorname{Id}_{L((z))}(\tau)=V\right)$. En effet si $M$ est le $\oslash \widehat{\otimes} \vartheta_{L}$-module libre associé à $N$ et $V$ comme dans le lemme 2.3, on a $\phi_{M}\left({ }^{\tau} M\right) \subset(z-\pi)^{s} M$ et $\phi_{M}^{-1}(M) \subset(z-\pi)^{-t \tau} M$ car $(z-\pi)^{s} \vartheta \widehat{\otimes} \vartheta_{L}\left[\frac{1}{z}\right] \cap L[[z]]=(z-\pi)^{s} \Theta \widehat{\otimes} \vartheta_{L}$ (et de même avec $\left.-t\right)$.

Montrons maintenant l'existence de $V$. Soit $V_{1}$ n'importe quel $L[[z]]$-réseau de

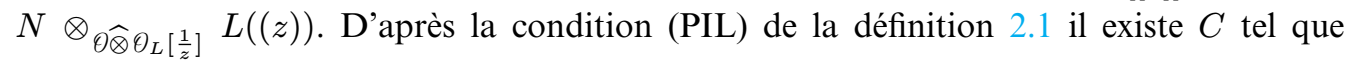
pour tout $n \in \mathbb{N}$ l'image de $\tau^{n} V_{1}$ par $\phi_{N}{ }^{\tau} \phi_{N} \ldots \tau^{n-1} \phi_{N}$ est comprise entre $z^{C} V_{1}$ et $z^{-C} V_{1}$. On pose alors

$$
V=\bigcup_{k \in \mathbb{N}} \bigcap_{n \in \mathbb{N}, n \geq k} \phi_{N}{ }^{\tau} \phi_{N} \cdots \tau^{n-1} \phi_{N}\left(\tau^{n} V_{1}\right)
$$

Il est clair que $V$ est un $L[[z]]$-réseau de $N \otimes_{\widehat{\vartheta}_{\widehat{\otimes} \vartheta_{L}\left[\frac{1}{z}\right]}} L((z))$, préservé par $\phi_{N} \otimes_{\vartheta \widehat{\otimes} \vartheta_{L}\left[\frac{1}{z-\pi}, \frac{1}{z}\right]} \operatorname{Id}_{L((z))}$.

Il serait intéressant d'étendre ce résultat à des bases plus générales (la démonstration du lemme 2.3 indique que des éclatements seraient nécessaires).

Lemme 2.3. - Soit $\theta_{L}$ un anneau de valuation (non nécessairement discrète), de corps des fractions $L$ (on ne suppose pas que $\theta_{L}$ est complet).

a) Soient $N$ un $\theta_{L}((z))$-module libre de rang $r, V$ un $L[[z]]$-module libre de rang $r$ et $\alpha: N \otimes_{\bigcirc_{L}((z))} L((z)) \rightarrow V \otimes_{L[[z]]} L((z))$ un isomorphisme de $L((z))$-modules libres. Alors il existe un unique triplet $(M, \beta, \gamma)$, où $M$ est un $\theta_{L}[[z]]$-module libre de rang $r, \beta: M \otimes_{\vartheta_{L}[[z]]} \vartheta_{L}((z)) \rightarrow N$ est un isomorphisme de $\theta_{L}((z))$-modules libres, $\gamma: M \otimes_{\vartheta_{L}[[z]]} L[[z]] \rightarrow V$ est un isomorphisme de $L[[z]]$-modules libres, et $\alpha \circ(\beta \otimes 1)=\gamma \otimes 1$.

b) Soient $M$ et $M^{\prime}$ des $\theta_{L}[[z]]$-modules libres, et

$$
\begin{aligned}
g & \in \operatorname{Hom}_{\vartheta_{L}((z))}\left(M \otimes_{\vartheta_{L}[[z]]} \vartheta_{L}((z)), M^{\prime} \otimes_{\vartheta_{L}[[z]]} \vartheta_{L}((z))\right) \\
\text { et } & h \in \operatorname{Hom}_{L[[z]]}\left(M \otimes_{\vartheta_{L}[[z]]} L[[z]], M^{\prime} \otimes_{\vartheta_{L}[[z]]} L[[z]]\right)
\end{aligned}
$$

tels que $g \otimes 1$ et $h \otimes 1$ coïncident dans

$$
\operatorname{Hom}_{L((z))}\left(M \otimes_{\vartheta_{L}[[z]]} L((z)), M^{\prime} \otimes_{\vartheta_{L}[[z]]} L((z))\right) .
$$

Alors il existe un unique morphisme $f: M \rightarrow M^{\prime}$ tel que $g=f \otimes 1$ et $h=f \otimes 1$.

$4^{\text {e }}$ SÉRIE - TOME $44-2011$ - No 2 
Lorsque $\theta_{L}$ est de valuation discrète, ce lemme est une variation du résultat selon lequel un fibré sur une surface régulière moins un point se prolonge de manière unique en un fibré sur la surface (cf. [44]) et il résulte du fait que tout module de type fini réflexif sur un anneau local régulier de dimension 2 est libre (lemme 6 de [54]).

Démonstration. - Partant d'un $\vartheta_{L}[[z]]$-module libre $M_{0}$ de rang $r$, muni d'un isomorphisme $M_{0} \otimes_{\vartheta_{L}[[z]]} \vartheta_{L}((z)) \simeq N$, il s'agit de le modifier au point générique du diviseur $z=0$. On peut se limiter à traiter le cas d'une modification élémentaire supérieure, c'est-à-dire que l'on suppose

$$
M_{0} \otimes_{\vartheta_{L}[[z]]} L[[z]] \subset V \subset z^{-1} M_{0} \otimes_{\vartheta_{L}[[z]]} L[[z]]
$$

avec $V / M_{0} \otimes_{\vartheta_{L}[[z]]} L[[z]]$ un $L$-espace vectoriel de dimension 1 et on doit montrer que $z^{-1} M_{0} \cap V$ est un $\vartheta_{L}[[z]]$-module libre de rang $r$. D'abord $V / M_{0} \otimes_{\vartheta_{L}[[z]]} L[[z]]$ est une droite du $L$-espace vectoriel (de rang $r)\left(z^{-1} M_{0} / M_{0}\right) \otimes_{\vartheta_{L}} L$. Il est évident que l'intersection de $\theta_{L}^{r}$ avec une droite de $L^{r}$ est un sous-module libre de rang 1 facteur direct de $\theta_{L}^{r}$ : il est engendré par un générateur de cette droite dont le minimum des valuations des coordonnées est nul. Donc il existe une base $e_{1}, \ldots, e_{r}$ du $\theta_{L}$-module libre $z^{-1} M_{0} / M_{0}$ telle que $L e_{1}=V / M_{0} \otimes_{\vartheta_{L}[[z]]} L[[z]]$. Soit $f_{1}, . ., f_{r}$ une base du $\vartheta_{L}[[z]]$-module libre $M_{0}$ telle que $z^{-1} f_{i} \bmod M_{0}=e_{i}$ pour $i=1, \ldots, r$. Alors $\left(z^{-1} f_{1}, f_{2}, \ldots, f_{r}\right)$ est une base du $\vartheta_{L}[[z]]$-module $z^{-1} M_{0} \cap V$ et celui-ci est donc libre de rang $r$.

\section{Structures de Hodge-Pink sur une base sans torsion}

Nous allons associer une structure de Hodge-Pink à un pseudo-isochtouca local sur un $\theta$-schéma formel $\pi$-adique plat. Il suffira de mener à bien cette construction dans le cas où la base est affine et où le pseudo-isochtouca local est libre.

Soit $B$ une $\theta$-algèbre complète pour la topologie $\pi$-adique et sans $\pi$-torsion. On dit qu'un pseudo-isochtouca local sur $B$ est libre s'il est libre comme $\theta \widehat{\otimes} B\left[\frac{1}{z}\right]$-module.

Soit $\mathscr{G}(B)$ le complété de $\theta \widehat{\otimes} B\left[\frac{1}{z}\right]$ pour la topologie $\pi$-adique (c'est-à-dire la topologie relative à l'idéal $\theta \widehat{\otimes} \pi B\left[\frac{1}{z}\right]$ de $\theta \widehat{\otimes} B\left[\frac{1}{z}\right]$ ). Nous commençons par en donner une autre description.

Pour $b \in B$ on note $\nu(b)=\sup \left\{k \in \mathbb{N}, b \in \pi^{k} B\right\} \in \mathbb{N} \cup\{+\infty\}$ : ce n'est pas une valuation, mais on a $\nu\left(b+b^{\prime}\right) \geq \min \left(\nu(b), \nu\left(b^{\prime}\right)\right)$ et $\nu\left(b b^{\prime}\right) \geq \nu(b)+\nu\left(b^{\prime}\right)$ pour $b, b^{\prime} \in B$ et $\nu(b)=+\infty$ si et seulement si $b=0$. On peut dire que $\nu$ est une sous-valuation, ou une norme additive.

Remarque. - Le lecteur peut être plus familier avec $\nu^{\prime}: B \rightarrow \mathbb{R}_{+} \cup\{+\infty\}$ défini par $\nu^{\prime}(b)=\inf _{\left(A, v_{A}, u\right)} v_{A}(u(b))$, où $\left(A, v_{A}, u\right)$ parcourt tous les anneaux de valuation réelle $\left(A, v_{A}\right)$ munis d'un morphisme $u: B \rightarrow A$ tel que $v_{A}(u(\pi))=1$. On a $\nu^{\prime} \geq \nu$. D'après le théorème 1 du paragraphe 6.2.4 de [10], si $B$ est topologiquement de type fini et réduite, $\nu^{\prime}-\nu$ est borné sur $B \backslash\{0\}$. Nous n'utiliserons pas $\nu^{\prime}$ dans ce paragraphe, car cela entraînerait des complications techniques.

Dans la suite on utilisera aussi $\nu: B\left[\frac{1}{\pi}\right] \rightarrow \mathbb{Z} \cup\{+\infty\}$ définie par $\nu(b)=\sup \{k \in \mathbb{Z}$, $\left.b \in \pi^{k} B\right\}$.

Alors

$$
\mathscr{G}(B)=\left\{\sum_{n \in \mathbb{Z}} b_{n} z^{n}, b_{n} \in B, \nu\left(b_{-n}\right) \text { tend vers }+\infty \text { quand } n \text { tend vers }+\infty\right\}
$$


et les $\pi^{k} \mathscr{G}(B)$ pour $k \in \mathbb{N}$ forment une base de voisinages de 0 dans $\mathscr{G}(B)$. Ensuite on pose

$$
\mathscr{C}(B)=\left\{\sum_{n \in \mathbb{Z}} b_{n} z^{n}, b_{n} \in B, \frac{\nu\left(b_{-n}\right)}{n} \text { tend vers }+\infty \text { quand } n \text { tend vers }+\infty\right\} .
$$

On a les inclusions $\theta \widehat{\otimes} B\left[\frac{1}{z}\right] \subset \mathscr{C}(B) \subset \mathscr{G}(B)$.

Remarque. - On a une inclusion $\iota: \mathscr{C}(B) \rightarrow \mathscr{C}^{\prime}(B)$ où $\mathscr{C}^{\prime}(B)$ est défini comme $\mathscr{C}(B)$ mais avec $\nu^{\prime}$ au lieu de $\nu$. On peut aussi voir $\mathscr{C}^{\prime}(B)$ comme le sous-anneau de $\mathscr{C}(B)$ formé des séries à coefficients dans $B$ en la variable $z$ et son inverse, qui convergent absolument sur la variété rigide $\operatorname{Spm} B\left[\frac{1}{\pi}\right] \times_{\operatorname{Spm} K}\{0<|z|<1\}$. Si $B$ est topologiquement de type fini et réduite, $\iota$ est un isomorphisme.

On introduit $\alpha=\left(1-\frac{\pi}{z}\right)\left(1-\frac{\pi^{q}}{z}\right)\left(1-\frac{\pi^{q^{2}}}{z}\right) \cdots \in \mathscr{C}(B)$. Comme $\alpha=1$ modulo $\pi, \alpha$ est une unité dans $\mathscr{C}(B)$, donc $\alpha$ n'est pas diviseur de 0 dans $\mathscr{C}(B)$.

Pour $y=\sum_{n \in \mathbb{N}} b_{n} z^{n} \in \mathscr{G}(B)$, et pour $w \in \mathbb{R}_{+}$, on pose

$$
\nu_{w}^{\text {naïf }}(y)=\inf _{n \in \mathbb{Z}} n w+\nu\left(b_{n}\right) \in \mathbb{R} \cup\{-\infty\} \cup\{+\infty\} .
$$

Ce n'est pas une valuation sur $\mathscr{G}(B)$, mais pour $y, y^{\prime} \in \mathscr{G}(B)$ on a

$$
\nu_{w}^{\text {naiif }}\left(y+y^{\prime}\right) \geq \min \left(\nu_{w}^{\text {naif }}(y), \nu_{w}^{\text {naif }}\left(y^{\prime}\right)\right) \text { et } \nu_{w}^{\text {naif }}\left(y y^{\prime}\right) \geq \nu_{w}^{\text {naif }}(y)+\nu_{w}^{\text {naiif }}\left(y^{\prime}\right)
$$

et $\nu_{w}^{\text {naif }}(y)=+\infty$ si et seulement si $y=0$. Par exemple, $\nu_{w}^{\text {naif }}\left(\tau^{n} \alpha^{-1}\right)=-\infty$ pour $w>q^{n}$, et $\nu_{w}^{\text {naif }}\left(\tau^{n} \alpha^{-1}\right)=0$ pour $w \leq q^{n}$. On a

$$
\mathscr{C}(B)=\left\{x \in \mathscr{G}(B), \forall w \in \mathbb{R}_{+}, \nu_{w}^{\text {naif }}(x)>-\infty\right\} .
$$

On munit $\mathscr{C}(B)$ de la topologie telle que les parties

$$
\left\{x \in \pi^{k} \mathscr{C}(B), \nu_{w}^{\text {naif }}(x) \geq k\right\}=\left\{x \in \mathscr{C}(B), \nu_{w}^{\text {naif }}(x) \geq k \text { et } \nu_{0}^{\text {naif }}(x) \geq k\right\}
$$

pour $k \in \mathbb{N}$ et $w \in \mathbb{R}_{+}$forment une base de voisinage de 0 .

Remarque. - L'inclusion $\mathscr{C}(B) \rightarrow \mathscr{A}(B)$ est continue. L'inclusion $\iota: \mathscr{C}(B) \rightarrow \mathscr{C}^{\prime}(B)$ est continue si on munit $\mathscr{C}^{\prime}(B)$ de la topologie de la convergence uniforme sur les couronnes $\{\alpha<|z|<1\}$ pour $\alpha \in] 0,1[$.

Lemme 3.1. - Si $\left(y_{n}\right)_{n \in \mathbb{N}}$ est une suite d'éléments de $\mathscr{C}(B)$ telle que pour tout $w \in \mathbb{R}_{+}$la suite $\nu_{w}^{\text {naif }}\left(y_{n}-y_{n+1}\right)$ tend vers $+\infty$, alors la suite $\left(y_{n}\right)$ converge dans $\mathscr{C}(B)$ vers une limite $y$ et pour tout $w \in \mathbb{R}_{+}, \nu_{w}^{\text {naif }}(y)=\lim _{n \rightarrow \infty} \nu_{w}^{\text {naif }}\left(y_{n}\right)$.

Le lemme précédent énonce que toute suite de Cauchy converge, c'est-à-dire que $\mathscr{C}(B)$ est complet.

On a un morphisme injectif $c_{\pi}: \mathscr{C}(B) \rightarrow\left(B\left[\frac{1}{\pi}\right]\right)[[z-\pi]]$ qui envoie $z \operatorname{sur} \pi+(z-\pi)$.

Remarque. - Si $B$ est topologiquement de type fini et réduite, le morphisme $c_{\pi}$ est le composé de l'isomorphisme $\iota: \mathscr{C}(B) \rightarrow \mathscr{C}^{\prime}(B)$ et du morphisme de restriction à un voisinage formel de $\pi$ dans le disque épointé, de $\mathscr{C}^{\prime}(B)$ (considéré comme un espace de fonctions sur $\left.\operatorname{Spm} B\left[\frac{1}{\pi}\right] \times \operatorname{Spm} K\{0<|z|<1\}\right)$ vers $\left(B\left[\frac{1}{\pi}\right]\right)[[z-\pi]]$.

On appelle topologie $\pi$-adique sur $B\left[\frac{1}{\pi}\right]$ la topologie pour laquelle les $\pi^{k} B, k \in \mathbb{N}$, forment une base de voisinages de 0 . 
Lemme 3.2. - Pour tout $k \in \mathbb{N}^{*}$ le morphisme

$$
\mathscr{C}(B) \rightarrow\left(B\left[\frac{1}{\pi}\right]\right)[[z-\pi]] /(z-\pi)^{k}\left(B\left[\frac{1}{\pi}\right]\right)[[z-\pi]]
$$

induit par $c_{\pi}$ est continu lorsque l'on munit l'espace d'arrivée, qui est un $B\left[\frac{1}{\pi}\right]$-module libre de rang $k$, de la topologie $\pi$-adique.

Le morphisme $c_{\pi}$ s'étend en un morphisme $\mathscr{C}(B)\left[\frac{1}{\alpha}\right] \rightarrow\left(B\left[\frac{1}{\pi}\right]\right)((z-\pi))$ que l'on note encore $c_{\pi}$.

Le a) de la proposition suivante est une variante du lemme de rigidité de Drinfeld (lemme 3 de l'appendice de [17] et lemme 1.1.3 de [37]).

Proposition 3.3. - Soient $N$ et $N^{\prime}$ des pseudo-isochtoucas locaux libres sur $B$, et $\rho$ un morphisme de pseudo-isochtoucas locaux de la restriction de $N$ à $B / \pi$ vers la restriction de $N^{\prime}$ $\grave{a} B / \pi$.

a) Il existe un unique morphisme $R$ de $\mathscr{C}(B)\left[\frac{1}{\alpha}\right]$-modules congru à $\rho$ modulo $\pi$ de $N \otimes_{\bigcirc \widehat{\otimes} B\left[\frac{1}{z}\right]} \mathscr{C}(B)\left[\frac{1}{\alpha}\right]$ vers $N^{\prime} \otimes_{\widehat{\partial} B\left[\frac{1}{z}\right]} \mathscr{C}(B)\left[\frac{1}{\alpha}\right]$ vérifiant $R\left(\phi_{N} \otimes 1\right)=\left(\phi_{N^{\prime}} \otimes 1\right)^{\tau} R$.

b) Le morphisme $R$ défini en a) est un morphisme de pseudo-isochtoucas locaux de $N$ vers $N^{\prime}$ si et seulement si $R$ n'a pas de pôle en $z=\pi$, c'est-à-dire si l'image (par $c_{\pi}$ ) de

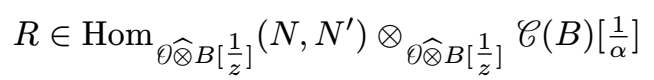

dans

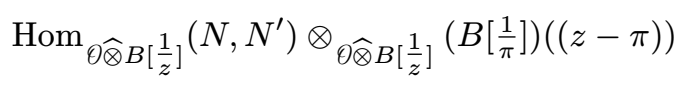

appartient à

$$
\operatorname{Hom}_{\oslash \widehat{\otimes} B\left[\frac{1}{z}\right]}\left(N, N^{\prime}\right) \otimes_{\vartheta \widehat{\otimes} B\left[\frac{1}{z}\right]}\left(B\left[\frac{1}{\pi}\right]\right)[[z-\pi]] .
$$

Remarque. - La construction de $\mathscr{C}(B)$ est compatible à la localisation. Donc l'énoncé s'étend à tout schéma formel $\pi$-adique plat sur Spf $\theta$ de la façon suivante : $B \mapsto \mathscr{C}(B)$ est un préfaisceau et si on note $\mathscr{C}_{S}$ le faisceau associé on construit $R \in \operatorname{Hom}_{\emptyset \widehat{\otimes} \vartheta_{S}\left[\frac{1}{z}\right]}\left(N, N^{\prime}\right)$ $\otimes_{\vartheta \widehat{\otimes} \vartheta_{S}\left[\frac{1}{z}\right]} \mathscr{C}_{S}\left[\frac{1}{\alpha}\right]$ (l'exposant de $\alpha$ est borné car il dépend seulement de l'amplitude).

Démonstration. - Nous montrons d'abord a), sans utiliser la condition (PIL) de la définition 2.1.

Pour l'unicité, on remarque que si $R$ est un morphisme de $\mathscr{C}(B)$-modules de $N \otimes_{\oslash \widehat{\otimes} B\left[\frac{1}{z}\right]}$

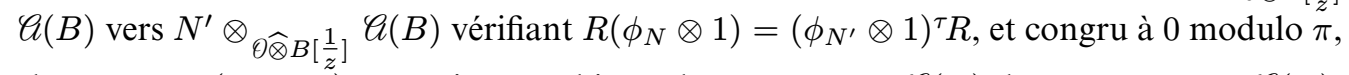
alors comme $\left(\phi_{N} \otimes 1\right)$ est un isomorphisme de ${ }^{\tau} N \otimes_{\partial \widehat{\otimes} B\left[\frac{1}{z}\right]} \mathscr{C}(B)$ dans $N \otimes_{\partial \widehat{\otimes} B\left[\frac{1}{z}\right]} \mathscr{C}(B)$, $R$ est congru à 0 modulo $\pi^{q}$, puis modulo $\pi^{q^{2}}, \ldots$ et enfin $R=0$.

L'existence de $R$ est un énoncé local pour la topologie de Zariski sur Spf $B$. On suppose donc que $N$ et $N^{\prime}$ sont des $\theta \widehat{\otimes} B\left[\frac{1}{z}\right]$-modules libres, ce qui permet

- de choisir des $\theta \widehat{\otimes} B$-modules libres $M$ et $M^{\prime}$ et des isomorphismes $M\left[\frac{1}{z}\right]=N$ et $M^{\prime}\left[\frac{1}{z}\right]=N^{\prime}$,

- de relever $\rho$ en $r_{0} \in \operatorname{Hom}_{\partial \widehat{\otimes} B\left[\frac{1}{z}\right]}\left(N, N^{\prime}\right)$. 


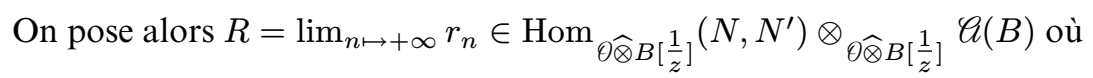

$$
r_{n}=\phi_{N^{\prime}} \ldots \tau^{n-1} \phi_{N^{\prime}} \tau^{n} r_{0} \tau^{n-1} \phi_{N}^{-1} \cdots \phi_{N}^{-1} \in \operatorname{Hom}_{\partial \widehat{\otimes} B\left[\frac{1}{z}\right]}\left(N, N^{\prime}\right)\left[\frac{1}{z-\pi}, \ldots, \frac{1}{z-\pi^{q^{n-1}}}\right] .
$$

Cette limite existe car $r_{n+1}-r_{n}=\phi_{N^{\prime}} \ldots \tau^{n-1} \phi_{N^{\prime}} \tau^{n}\left(\phi_{N^{\prime}} r_{0} \phi_{N}^{-1}-r_{0}\right) \tau^{n-1} \phi_{N}^{-1} \cdots \phi_{N}^{-1}$

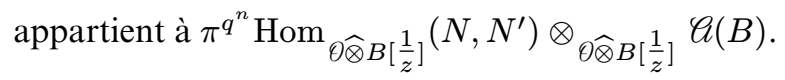

Soit $C$ une constante telle que $\phi_{N^{\prime}} \in z^{-C}(z-\pi)^{-C} \operatorname{Hom}_{\vartheta \widehat{\otimes} B}\left({ }^{\tau} M^{\prime}, M^{\prime}\right)$, $\phi_{N}^{-1} \in z^{-C}(z-\pi)^{-C} \operatorname{Hom}_{\vartheta \widehat{\otimes} B}\left(M,{ }^{\tau} M\right) \quad$ et $\quad r_{0} \in z^{-C} \operatorname{Hom}_{\vartheta \widehat{\otimes} B}\left(M, M^{\prime}\right)$. Comme $\pi^{q^{n}} \mathscr{G}(B) \cap \Theta \widehat{\otimes} B=\pi^{q^{n}} \Theta \widehat{\otimes} B$ on a

$$
z^{C(2 n+3)}(z-\pi)^{2 C} \cdots\left(z-\pi^{q^{n}}\right)^{2 C}\left(r_{n+1}-r_{n}\right) \in \pi^{q^{n}} \operatorname{Hom}_{\widehat{\vartheta} \widehat{\otimes} B}\left(M, M^{\prime}\right)
$$

donc

$$
\alpha^{2 C}\left(r_{n+1}-r_{n}\right) \in z^{-C(4 n+5)} \pi^{q^{n}}\left(\tau^{n+1} \alpha\right)^{2 C} \operatorname{Hom}_{\partial \widehat{\otimes} B}\left(M, M^{\prime}\right)
$$

et donc

$$
\alpha^{2 C}\left(r_{n+1}-r_{n}\right) \in z^{-C(4 n+5)} \pi^{q^{n}} \operatorname{Hom}_{\widehat{\partial} \widehat{\otimes} B}\left(M, M^{\prime}\right) \otimes_{\vartheta \widehat{\otimes} B} \oslash \widehat{\otimes} B\left[\left[\frac{\pi^{q^{n+1}}}{z}\right]\right] .
$$

Il résulte du lemme 3.1 que la suite $\alpha^{2 C} r_{n}$ converge dans

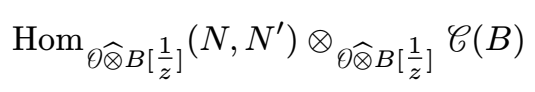

et donc $R$ appartient à

$$
\operatorname{Hom}_{\oslash \widehat{\otimes} B\left[\frac{1}{z}\right]}\left(N, N^{\prime}\right) \otimes_{\oslash \widehat{\otimes} B\left[\frac{1}{z}\right]} \alpha^{-2 C} \mathscr{C}(B) .
$$

Ceci termine la démonstration de a).

Pour montrer b) on utilise la condition (PIL) de la définition 2.1. Le «seulement si » est trivial. La preuve de «si » s'étend jusqu'au lemme 3.7. Pour tout $n \in \mathbb{N}$, on a un morphisme d'algèbres $c_{\pi^{q^{n}}}: \mathscr{C}(B) \rightarrow\left(B\left[\frac{1}{\pi}\right]\right)\left[\left[z-\pi^{q^{n}}\right]\right]$ (induit par la restriction au voisinage formel de $\pi^{q^{n}}$ dans le disque épointé $\{0<|z|<1\}$ ), qui s'étend en un morphisme $\mathscr{C}(B)\left[\frac{1}{\alpha}\right] \rightarrow\left(B\left[\frac{1}{\pi}\right]\right)\left(\left(z-\pi^{q^{n}}\right)\right)$. Par hypothèse $R$ n'a pas de pôle en $z=\pi$ donc $\tau^{n} R$ n'a pas de pôle en $z=\pi^{q^{n}}$ et comme $\phi_{N}^{-1}$ et $\phi_{N^{\prime}}$ n'ont pas de pôle en $z=\pi^{q}, \ldots, \pi^{q^{n}}$, $R=\phi_{N^{\prime}} \ldots \tau^{n-1} \phi_{N^{\prime}} \tau^{n} R^{\tau^{n-1}} \phi_{N}^{-1} \cdots \phi_{N}^{-1}$ n'a pas de pôle en $z=\pi^{q^{n}}$, c'est-à-dire que

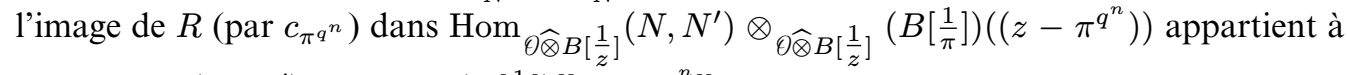

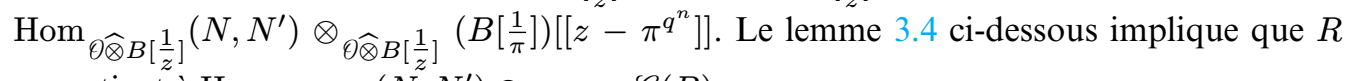

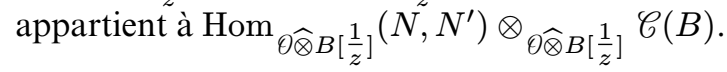

Lemme 3.4. - Soit $x \in \mathscr{C}(B)\left[\frac{1}{\alpha}\right]$ tel que, pour tout $n \in \mathbb{N}$, l'image de $x$ dans $\left(B\left[\frac{1}{\pi}\right]\right)\left(\left(z-\pi^{q^{n}}\right)\right)$ appartienne $\grave{a}\left(B\left[\frac{1}{\pi}\right]\right)\left[\left[z-\pi^{q^{n}}\right]\right]$. Alors $x$ appartient à $\mathscr{C}(B)$.

REMARQue. - L'énoncé analogue pour $\mathscr{C}^{\prime}(B)$ est évident.

Démonstration du lemme 3.4. - Le b) du lemme suivant renforce le lemme 3.4. 
Lemme 3.5. - a) Soit $n \in \mathbb{N}$. Si l'image de $x \in \mathscr{C}(B)$ dans $\left(B\left[\frac{1}{\pi}\right]\right)\left[\left[z-\pi^{q^{n}}\right]\right]$ appartient à $\left(z-\pi^{q^{n}}\right)\left(B\left[\frac{1}{\pi}\right]\right)\left[\left[z-\pi^{q^{n}}\right]\right]$, alors $x=\left(1-\frac{\pi^{q^{n}}}{z}\right) y$, avec $y \in \mathscr{C}(B)$, et pour tout $w \in \mathbb{R}_{+}$on $a$

$$
\nu_{w}^{\text {naif }}(y)=\nu_{w}^{\text {naïf }}(x)-\min \left(0, q^{n}-w\right)=\nu_{w}^{\text {naif }}(x)-\nu_{w}^{\text {naiff }}\left(1-\frac{\pi^{q^{n}}}{z}\right) .
$$

b) Soit $x \in \mathscr{C}(B)$ tel que, pour tout $n \in \mathbb{N}$, l'image de $x$ dans $\left(B\left[\frac{1}{\pi}\right]\right)\left[\left[z-\pi^{q^{n}}\right]\right]$ appartienne à $\left(z-\pi^{q^{n}}\right)\left(B\left[\frac{1}{\pi}\right]\right)\left[\left[z-\pi^{q^{n}}\right]\right]$. Alors $x=\alpha y$ avec $y \in \mathscr{C}(B)$ et pour tout $w \in \mathbb{R}_{+}$on $a$

$$
\nu_{w}^{\text {naif }}(y)=\nu_{w}^{\text {naif }}(x)-\sum_{n=0}^{\infty} \min \left(0, q^{n}-w\right)=\nu_{w}^{\text {naïf }}(x)-\nu_{w}^{\text {naif }}(\alpha) .
$$

Démonstration. - Montrons d'abord a). A priori y est dans $\mathscr{C}(B)$. Grâce à (1) l'appartenance de $y$ à $\mathscr{C}(B)$ résulte de la formule pour $\nu_{w}^{\text {naif }}(y)$, que nous allons établir. D'abord l'inégalité $\nu_{w}^{\text {naïf }}(y) \leq \nu_{w}^{\text {naif }}(x)-\nu_{w}^{\text {naiff }}\left(1-\frac{\pi^{q^{n}}}{z}\right)$ est une propriété générale. Nous devons montrer l'inégalité inverse. Posons $x=\sum_{m \in \mathbb{Z}} b_{m} z^{m}$. L'appartenance de $x$ à $\left(z-\pi^{q^{n}}\right)\left(B\left[\frac{1}{\pi}\right]\right)\left[\left[z-\pi^{q^{n}}\right]\right]$ équivaut à l'égalité

$$
\sum_{m \in \mathbb{Z}} b_{m}\left(\pi^{q^{n}}\right)^{m}=0 \text { dans } B\left[\frac{1}{\pi}\right]
$$

On a alors $y=\left(1-\frac{\pi^{q^{n}}}{z}\right)^{-1} x$, donc $y=\sum_{m \in \mathbb{Z}} c_{m} z^{m}$, avec

$$
c_{m}=b_{m}+\left(\pi^{q^{n}}\right) b_{m+1}+\left(\pi^{q^{n}}\right)^{2} b_{m+2}+\cdots
$$

Grâce à (2) on a aussi

$$
c_{m}=-\left(\pi^{q^{n}}\right)^{-1} b_{m-1}-\left(\pi^{q^{n}}\right)^{-2} b_{m-2}-\cdots
$$

Dans le cas où $w \leq q^{n}$, la formule (3) donne

$$
\begin{aligned}
m w+\nu\left(c_{m}\right) & \geq \inf \left(m w+\nu\left(b_{m}\right), m w+q^{n}+\nu\left(b_{m+1}\right), \ldots\right) \\
& \geq \inf \left(\nu_{w}^{\text {naiff }}(x), \nu_{w}^{\text {naiff }}(x)+q^{n}-w, \ldots\right)=\nu_{w}^{\text {naif }}(x) .
\end{aligned}
$$

Dans le cas où $w>q^{n}$, la formule (4) donne

$$
\begin{aligned}
m w+\nu\left(c_{m}\right) & \geq \inf \left(m w-q^{n}+\nu\left(b_{m-1}\right), m w-2 q^{n}+\nu\left(b_{m-2}\right), \ldots\right) \\
& \geq \inf \left(\nu_{w}^{\text {naif }}(x)-q^{n}+w, \nu_{w}^{\text {naif }}(x)-2 q^{n}+2 w, \ldots\right)=\nu_{w}^{\text {naif }}(x)-q^{n}+w .
\end{aligned}
$$

Montrons maintenant le b). Grâce à a), pour tout entier $n \in \mathbb{N}$ on peut écrire $x=\left(1-\frac{\pi}{z}\right) \cdots\left(1-\frac{\pi^{q^{n-1}}}{z}\right) y_{n}$ avec $y_{n} \in \mathscr{C}(B)$ et, pour tout $w \in \mathbb{R}_{+}, \nu_{w}^{\text {naif }}\left(y_{n}\right) \geq \nu_{w}^{\text {naif }}(x)$. On a $y_{n}=\left(1-\frac{\pi^{q^{n}}}{z}\right) y_{n+1}$ donc $y_{n}-y_{n+1}=-\frac{\pi^{q^{n}}}{z} y_{n+1}$ et $\nu_{w}^{\text {naif }}\left(y_{n}-y_{n+1}\right) \geq q^{n}-w+\nu_{w}^{\text {naif }}(x)$. Donc par le lemme 3.1 la suite $\left(y_{n}\right)$ converge dans $\mathscr{C}(B)$ et on note $y$ sa limite. Soit $w \in \mathbb{R}_{+}$. D'après a) et le lemme 3.1 on a

$$
\nu_{w}^{\text {naiff }}\left(y_{n}\right)=\nu_{w}^{\text {naif }}(x)-\sum_{m=0}^{n-1} \min \left(0, q^{m}-w\right) \text { et } \nu_{w}^{\text {naïf }}(y)=\lim _{n \mapsto \infty} \nu_{w}^{\text {naif }}\left(y_{n}\right),
$$

d'où la formule pour $\nu_{w}^{\text {naiff }}(y)$. Le lemme 3.5 est démontré et donc le lemme 3.4 l'est également. 
Le lemme 3.5 permet d'étendre $\nu_{w}$ de $\mathscr{C}(B)$ à $\mathscr{C}(B)\left[\frac{1}{\alpha}\right]$. Cela fait l'objet de la définition suivante.

Définition 3.6. - On définit $\nu_{w}: \mathscr{C}(B)\left[\frac{1}{\alpha}\right] \rightarrow \mathbb{R} \cup\{+\infty\}$ en posant, pour $x \in \alpha^{-t} \mathscr{C}(B)$, $\nu_{w}(x)=\nu_{w}^{\text {naif }}\left(\alpha^{t} x\right)-t \nu_{w}^{\text {naiff }}(\alpha)$ (grâce au lemme 3.5, le résultat ne dépend pas de $\left.t\right)$.

On note que la restriction de $\nu_{w}^{\text {naif }}$ à $\mathscr{C}(B)\left[\frac{1}{\alpha}\right] \subset \mathscr{G}(B)$ n'est pas égale à $\nu_{w}$ (car elle prend souvent la valeur $-\infty$ ) d'où l'adjectif «naïf».

Dans la suite on utilisera souvent la convention suivante : si $M$ et $M^{\prime}$ sont des

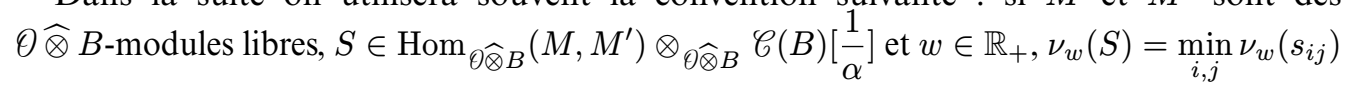
où les $s_{i j}$ sont les coefficients de $S$ dans des bases de $M$ et $M^{\prime}$ sur $\Theta \widehat{\otimes} B$ (il est clair que $\nu_{w}(S)$ ne dépend pas du choix de ces bases).

Suite de la démonstration de la proposition 3.3. - On sait maintenant que $R$ appartient à

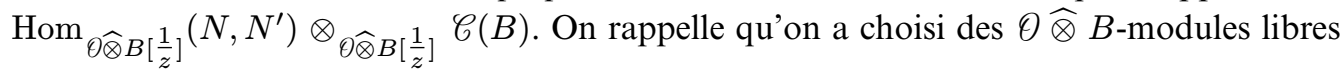
$M$ et $M^{\prime}$ munis d'isomorphismes $M\left[\frac{1}{z}\right]=N$ et $M^{\prime}\left[\frac{1}{z}\right]=N^{\prime}$. D'après la condition (PIL) de la définition 2.1, il existe une constante $C$, telle que pour tout $n \in \mathbb{N}^{*}, \phi_{N^{\prime}}{ }^{\tau} \phi_{N^{\prime}} \ldots \tau^{n-1} \phi_{N^{\prime}}$ appartienne à

$$
z^{-C}(z-\pi)^{-C}\left(z-\pi^{q}\right)^{-C} \cdots\left(z-\pi^{q^{n-1}}\right)^{-C} \operatorname{Hom}_{\emptyset \widehat{\otimes} B}\left(\tau^{n} M^{\prime}, M^{\prime}\right)
$$

et que $\left(\phi_{N}^{\tau} \phi_{N} \ldots \tau^{n-1} \phi_{N}\right)^{-1}$ appartienne à

$$
z^{-C}(z-\pi)^{-C}\left(z-\pi^{q}\right)^{-C} \cdots\left(z-\pi^{q^{n-1}}\right)^{-C} \operatorname{Hom}_{\widehat{\partial} \widehat{\otimes} B}\left(M,{ }^{n} M\right) .
$$

Soit $n \in \mathbb{N}$. On a

$$
\begin{aligned}
z^{2 C}(z-\pi)^{2 C} \cdots & \left(z-\pi^{q^{n-1}}\right)^{2 C} R \\
= & \left(z^{C}(z-\pi)^{C} \cdots\left(z-\pi^{q^{n-1}}\right)^{C} \phi_{N^{\prime}}{ }^{\tau} \phi_{N^{\prime}} \ldots \tau^{n-1} \phi_{N^{\prime}}\right) \\
& \tau^{n} R\left(z^{C}(z-\pi)^{C} \cdots\left(z-\pi^{q^{n-1}}\right)^{C} \tau^{n-1} \phi_{N}^{-1} \ldots{ }^{\tau} \phi_{N}^{-1} \phi_{N}^{-1}\right) .
\end{aligned}
$$

On a donc $\nu_{q^{n}}\left(z^{2 C}(z-\pi)^{2 C} \cdots\left(z-\pi^{q^{n-1}}\right)^{2 C} R\right) \geq \nu_{q^{n}}\left(\tau^{n} R\right) \geq q^{n} \nu_{1}(R)$. Le lemme 3.5 implique alors

$$
\nu_{q^{n}}(R) \geq q^{n} \nu_{1}(R)-2 C\left(q^{n}+q^{n-1}+\cdots+1\right) .
$$

Il existe donc une constante $C^{\prime} \in \mathbb{R}$ telle que pour tout $n \in \mathbb{N}$ on ait $\nu_{q^{n}}(R) \geq C^{\prime} q^{n}$. Le lemme suivant montre que $R$ a ses coefficients dans $\theta \widehat{\otimes} B\left[\frac{1}{z}\right]$, ce qui achève la démonstration de la proposition 3.3.

Lemme 3.7. - Soit $x \in \mathscr{C}(B)$. On suppose qu'il existe $C^{\prime} \in \mathbb{R}$ tel que $\nu_{q^{n}}(x) \geq C^{\prime} q^{n}$ pour tout $n \in \mathbb{N}$. Alors $x$ appartient à $\Theta \widehat{\otimes} B\left[\frac{1}{z}\right]$.

Démonstration. - On écrit $x=\sum_{m \in \mathbb{Z}} x_{m} z^{m}$. Soit $m \in \mathbb{Z}$. Pour tout $n \in \mathbb{N}$ on a donc $\nu\left(x_{m}\right) \geq C^{\prime} q^{n}-m q^{n}$, d'où $x_{m}=0$ si $C^{\prime}>m$. On a donc $x \in z^{-\left[-C^{\prime}\right]} \oslash \widehat{\otimes} B$ en notant [.] la partie entière. 
Dans toute la suite de ce paragraphe on fixe un corps parfait $k$ contenant $\mathbb{F}_{q}$ et on suppose que la base $S$ est un schéma $\pi$-adique plat sur Spf $\theta \widehat{\otimes} k$. On notera $S_{0}$ le fermé de $S$ d'idéal $\pi$ (comme $S$ est $\pi$-adique c'est en fait un schéma sur $k$ ).

Soit $\left(D, \phi_{D}\right)$ un isochtouca local sur $k$. Il jouera le rôle de $F$-isocristal, ou de module de Dieudonné rationnel pour rigidifier le pseudo-isochtouca local $N$, comme dans [51].

Définition 3.8. - Soit $\left(N, \phi_{N}\right)$ un pseudo-isochtouca local sur $S$. On appelle rigidification modulo $\pi$ un isomorphisme $\rho$ entre la restriction de $\left(N, \phi_{N}\right)$ à $S_{0}$ et l'image inverse de $\left(D, \phi_{D}\right) \grave{a} S_{0}$.

Alors à un pseudo-isochtouca local rigidifié $\left(\left(N, \phi_{N}\right), \rho\right)$ on va associer une structure de Hodge-Pink sur la fibre générique $S_{\text {rig }}$ de $S$ au sens de Raynaud.

Définition 3.9. - Soit \& une variété rigide analytique sur $\operatorname{Spm} K$. Soient $s, t \in \mathbb{Z}$. Une structure de Hodge-Pink sur $D$ définie sur \& d'amplitude $\subset[s, t]$ est un $\theta_{\phi}[[z-\pi]]$-module $V$ localement libre muni d'un isomorphisme

$$
V \otimes_{\vartheta_{\phi}[[z-\pi]]} \theta_{\phi}((z-\pi)) \simeq{ }^{\tau} D \otimes_{k((z))} \theta_{\phi}((z-\pi))
$$

et tel qu'en posant $U_{D}={ }^{\tau} D \otimes_{k((z))} \theta_{\&}[[z-\pi]]$ on ait

$$
(z-\pi)^{-s} U_{D} \subset V \subset(z-\pi)^{-t} U_{D}
$$

Le morphisme $k((z)) \rightarrow \vartheta_{\varnothing}[[z-\pi]]$ utilisé dans la définition précédente envoie $k$ dans $\theta_{\S}$ et $z$ sur $\pi+(z-\pi)$.

Remarque. - Dans la définition précédente $\theta_{\phi}[[z-\pi]]$ est un faisceau mais n'est pas quasi-cohérent et $\Theta_{\phi}((z-\pi))$ n'est qu'un préfaisceau. Voici, grâce au lemme 1.1, une version équivalente plus terre-à-terre de la définition : une structure de Hodge-Pink d'amplitude $\subset[s, t]$ est un sous- $\theta_{\S}$-module localement facteur direct et stable par multiplication par $z-\pi$

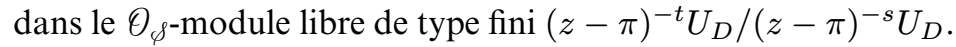

Pour construire la structure de Hodge-Pink associée à un pseudo-isochtouca on suppose d'abord que la base est affine et que le pseudo-isochtouca est libre. Soient donc $B$ une $\theta \widehat{\otimes} k$-algèbre complète pour la topologie $\pi$-adique et sans $\pi$-torsion, et $\left(N, \phi_{N}\right)$ un pseudoisochtouca local libre sur $B$.

Lemme 3.10. - Il existe un unique morphisme $R$ de $\mathscr{C}(B)\left[\frac{1}{\alpha}\right]$-modules congru à $\rho$ modulo $\pi$ de $N \otimes_{\partial \widehat{\otimes} B\left[\frac{1}{z}\right]} \mathscr{C}(B)\left[\frac{1}{\alpha}\right]$ vers $D \otimes_{k((z))} \mathscr{C}(B)\left[\frac{1}{\alpha}\right]$ vérifiant

$$
R\left(\phi_{N} \otimes 1\right)=\left(\phi_{D} \otimes 1\right)^{\tau} R .
$$

De plus c'est un isomorphisme de $\mathscr{C}(B)\left[\frac{1}{\alpha}\right]$-modules.

Démonstration. - L'unicité de $R$ se démontre comme dans a) de la proposition 3.3, en remplaçant $\phi_{N^{\prime}}$ par $\phi_{D}$. L'existence se démontre aussi comme dans a) de la proposition 3.3 grâce au fait que la condition (PIL) de la définition 2.1 (qui n'est pas vérifiée par $\phi_{D}$ ) n'y était pas utilisée. Cependant nous reprenons l'argument pour la commodité du lecteur.

Par hypothèse $N$ est un $\theta \widehat{\otimes} B\left[\frac{1}{z}\right]$-module libre, ce qui permet

- de choisir un $\theta \widehat{\otimes} B$-module libre $M$ avec un isomorphisme $M\left[\frac{1}{z}\right]=N$, ainsi qu'un $k[[z]]$-réseau $\Delta$ de $D$, 


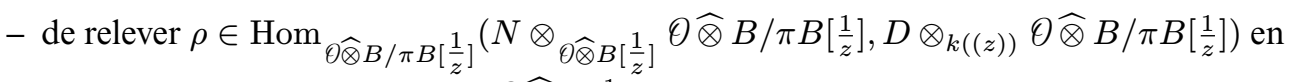
$r_{0} \in \operatorname{Hom}_{\emptyset \widehat{\otimes} B\left[\frac{1}{z}\right]}\left(N, D \otimes_{k((z))} \theta \widehat{\otimes} B\left[\frac{1}{z}\right]\right)$.

On pose alors

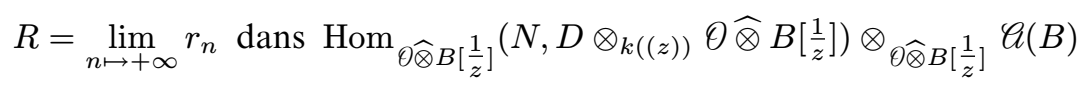

où

$r_{n}=\phi_{D} \cdots \tau^{n-1} \phi_{D} \tau^{n} r_{0}^{\tau^{n-1}} \phi_{N}^{-1} \cdots \phi_{N}^{-1} \in \operatorname{Hom}_{\oslash \widehat{\otimes} B\left[\frac{1}{z}\right]}\left(N, D \otimes_{k((z))} \oslash \widehat{\otimes} B\left[\frac{1}{z}\right]\right)\left[\frac{1}{z-\pi}, \ldots, \frac{1}{z-\pi^{q^{n-1}}}\right]$.

Cette limite existe car $r_{n+1}-r_{n}=\phi_{D} \cdots \tau^{n-1} \phi_{D} \tau^{n}\left(\phi_{D}{ }^{\tau} r_{0} \phi_{N}^{-1}-r_{0}\right) \tau^{n-1} \phi_{N}^{-1} \cdots \phi_{N}^{-1}$

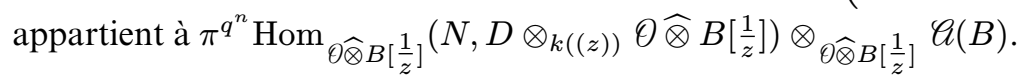

Soit $C$ une constante telle que $\phi_{N}^{-1} \in z^{-C}(z-\pi)^{-C} \operatorname{Hom}_{\partial \widehat{\otimes} B}\left(M,{ }^{\tau} M\right)$, $\phi_{D} \in z^{-C} \operatorname{Hom}_{k[[z]]}\left({ }^{\tau} \Delta, \Delta\right)$ et $r_{0} \in z^{-C} \operatorname{Hom}_{\vartheta \widehat{\otimes} B}\left(M, \Delta \otimes_{k[[z]]} \oslash \widehat{\otimes} B\right)$. Alors

$$
\left(r_{n+1}-r_{n}\right) \in z^{-C(2 n+3)}(z-\pi)^{-C} \cdots\left(z-\pi^{q^{n}}\right)^{-C} \pi^{q^{n}} \operatorname{Hom}_{\partial \widehat{\otimes} B}\left(M, \Delta \otimes_{k[[z]]} \oslash \widehat{\otimes} B\right)
$$

donc

$$
\alpha^{C}\left(r_{n+1}-r_{n}\right) \in z^{-C(3 n+4)} \pi^{q^{n}}\left(\tau^{n+1} \alpha\right)^{C} \operatorname{Hom}_{\vartheta \widehat{\otimes} B}\left(M, \Delta \otimes_{k[[z]]} \oslash \widehat{\otimes} B\right)
$$

et donc

$$
\alpha^{C}\left(r_{n+1}-r_{n}\right) \in z^{-C(3 n+4)} \pi^{q^{n}} \operatorname{Hom}_{\vartheta \widehat{\otimes} B}\left(M, \Delta \otimes_{k[[z]]} \oslash \widehat{\otimes} B\right) \otimes_{\vartheta \widehat{\otimes} B} \oslash \widehat{\otimes} B\left[\left[\frac{\pi^{q^{n+1}}}{z}\right]\right] .
$$

Le lemme 3.1 montre que la suite $\alpha^{C} r_{n}$ converge dans $\mathscr{C}(B)$. Donc $R$ appartient à $\operatorname{Hom}_{\oslash \widehat{\otimes} B\left[\frac{1}{z}\right]}\left(N, D \otimes_{k((z))} \oslash \widehat{\otimes} B\left[\frac{1}{z}\right]\right) \otimes_{\oslash \widehat{\otimes} B\left[\frac{1}{z}\right]} \alpha^{-C} \mathscr{C}(B)$. Ceci termine la démonstration de l'existence de $R$.

De la même façon, on choisit un relèvement $r_{0}^{\prime} \in \operatorname{Hom}_{\widehat{\partial} \widehat{\otimes} B\left[\frac{1}{z}\right]}\left(D \otimes_{k((z))} \oslash \widehat{\otimes} B\left[\frac{1}{z}\right], N\right)$ de $\rho^{-1}$, on pose $R^{\prime}=\lim _{n \mapsto+\infty} \phi_{N} \cdots \tau^{n-1} \phi_{N} \tau^{n} r_{0}^{\prime} \tau^{n-1} \phi_{D}^{-1} \cdots \phi_{D}^{-1}$ et on montre que $R^{\prime}$

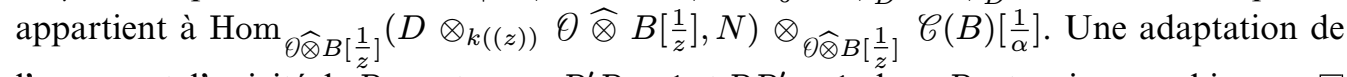
l'argument d'unicité de $R$ montre que $R^{\prime} R=1$ et $R R^{\prime}=1$, donc $R$ est un isomorphisme.

Nous allons maintenant définir la structure de Hodge-Pink sur $D$. Le morphisme $c_{\pi}: \mathscr{C}(B) \rightarrow\left(B\left[\frac{1}{\pi}\right]\right)[[z-\pi]]$ s'étend en des morphismes $\mathscr{C}(B)\left[\frac{1}{\tau_{\alpha}}\right] \rightarrow\left(B\left[\frac{1}{\pi}\right]\right)[[z-\pi]]$ et $\mathscr{C}(B)\left[\frac{1}{\alpha}\right] \rightarrow\left(B\left[\frac{1}{\pi}\right]\right)((z-\pi))$. Par conséquent $R$ s'étend en un isomorphisme de $\left(B\left[\frac{1}{\pi}\right]\right)((z-\pi))$-modules de $N \otimes_{\bigcirc \widehat{\otimes} B\left[\frac{1}{z}\right]}\left(B\left[\frac{1}{\pi}\right]\right)((z-\pi))$ vers $D \otimes_{k((z))}\left(B\left[\frac{1}{\pi}\right]\right)((z-\pi))$ et ${ }^{\tau} R$ s'étend en un isomorphisme de $\left(B\left[\frac{1}{\pi}\right]\right)[[z-\pi]]$-modules de ${ }^{\tau} N \otimes_{\vartheta \widehat{\otimes} B\left[\frac{1}{z}\right]}\left(B\left[\frac{1}{\pi}\right]\right)[[z-\pi]]$ vers ${ }^{\tau} D \otimes_{k((z))}\left(B\left[\frac{1}{\pi}\right]\right)[[z-\pi]]$.

Définition 3.11. - La structure de Hodge-Pink $V$ associée au pseudo-isochtouca local libre $\left(N, \phi_{N}\right)$ sur $B$ et à l'isomorphisme $\rho$ entre les pseudo-isochtoucas locaux

$$
\left(N \otimes_{\oslash \widehat{\otimes} B\left[\frac{1}{z}\right]} \oslash \widehat{\otimes}(B / \pi B)\left[\frac{1}{z}\right], \phi_{N} \otimes 1\right) \text { et }\left(D \otimes_{k((z))} \oslash \widehat{\otimes}(B / \pi B)\left[\frac{1}{z}\right], \phi_{D} \otimes 1\right)
$$


sur $B / \pi B$ est le $\left(B\left[\frac{1}{\pi}\right]\right)[[z-\pi]]$-sous-module (localement libre pour la topologie de Zariski sur $\operatorname{Spf} B)$ de ${ }^{\tau} D \otimes_{k((z))}\left(B\left[\frac{1}{\pi}\right]\right)((z-\pi))$ défini par

$$
\begin{aligned}
V & ={ }^{\tau} R\left(\phi_{N}^{-1}\left(N \otimes_{\vartheta \widehat{\otimes} B\left[\frac{1}{z}\right]}\left(B\left[\frac{1}{\pi}\right]\right)[[z-\pi]]\right)\right) \\
& =\left(\phi_{D} \otimes 1\right)^{-1}\left(R\left(N \otimes_{\vartheta \widehat{\otimes} B\left[\frac{1}{z}\right]}\left(B\left[\frac{1}{\pi}\right]\right)[[z-\pi]]\right)\right) .
\end{aligned}
$$

La construction précédente se faisceautise et permet d'associer à tout pseudo-isochtouca $\left(N, \phi_{N}\right)$ muni d'une rigidification $\rho \operatorname{par}\left(D, \phi_{D}\right)$ sur un schéma formel $\pi$-adique plat $S$ au-dessus de $\operatorname{Spf} \oslash \widehat{\otimes} k$ une structure de Hodge-Pink sur $D$ définie sur la fibre générique $S_{\text {rig }}$ de $S$ au sens de Raynaud.

On revient à la situation de la définition 3.11. La proposition 3.12 ci-dessous (qui est une forme plus explicite du b) de la proposition 3.3) montre que $V$ détermine de manière unique $R(N) \subset D \otimes_{k((z))} \mathscr{C}(B)\left[\frac{1}{\alpha}\right]$, et par conséquent détermine le couple $\left(\left(N, \phi_{N}\right), \rho\right)$ à un unique isomorphisme près.

Soit $\widetilde{N}$ l'ensemble des $x \in D \otimes_{k((z))} \mathscr{C}(B)\left[\frac{1}{\alpha}\right]$, tels que

- pour tout $n \in \mathbb{N}$, l'image de $x$ dans $D \otimes_{k((z))}\left(B\left[\frac{1}{\pi}\right]\right)\left(\left(z-\pi^{q^{n}}\right)\right)$ appartienne à $\phi_{D} \ldots \tau^{n} \phi_{D} \tau^{n} V$

- il existe une constante $C \in \mathbb{R}$ telle que pour tout $n \in \mathbb{N}$,

$$
\nu_{q^{n}}\left(\left(\phi_{D} \cdots \tau^{n-1} \phi_{D}\right)^{-1} x\right) \geq C q^{n} .
$$

Pour donner un sens à la dernière condition il faut choisir un réseau $\Delta$ de $D:$ dans une base sur $k[[z]] \mathrm{de}^{\tau^{n} \Delta}$ on écrit $\left(\phi_{D} \ldots \tau^{n-1} \phi_{D}\right)^{-1} x=\left(\begin{array}{c}y_{1} \\ \vdots \\ y_{r}\end{array}\right)$ avec $y_{i} \in \mathscr{C}(B)\left[\frac{1}{\alpha}\right]$ et on pose $\nu_{q^{n}}\left(\left(\phi_{D} \ldots \tau^{n-1} \phi_{D}\right)^{-1} x\right)=\min \left(\nu_{q^{n}}\left(y_{1}\right), \ldots, \nu_{q^{n}}\left(y_{r}\right)\right)$. Comme $\nu_{q^{n}}(z b)=q^{n}+\nu_{q^{n}}(b)$ pour tout $b \in \mathscr{C}(B)\left[\frac{1}{\alpha}\right]$, si on change $\Delta$ la dernière condition reste vraie pour une autre constante $C$, donc la définition de $\widetilde{N}$ ne dépend pas du choix d'un réseau $\Delta$ de $D$. On vérifie facilement que $\left(\phi_{D} \otimes 1\right)\left({ }^{\tau} \tilde{N}\left[\frac{1}{z-\pi}\right]\right)=\tilde{N}\left[\frac{1}{z-\pi}\right]$.

Proposition 3.12. - On a $R(N)=\widetilde{N}$.

Démonstration. - On suppose $\left(N, \phi_{N}\right)$ d'amplitude $\subset[s, t]$. Comme l'énoncé est local pour la topologie de Zariski sur Spf $B$ on peut supposer $N$ libre sur $\theta \widehat{\otimes} B\left[\frac{1}{z}\right]$. On fixe alors un $\Theta \widehat{\otimes} B$-module libre $M$ muni d'un isomorphisme $N=M\left[\frac{1}{z}\right]$ et une constante $C_{0}$ telle que la condition (PIL) de la définition 2.1 soit satisfaite (avec $C_{0}$ au lieu de $C$ ). On fixe aussi un réseau $\Delta$ de $D$. Grâce à $\Delta$ et $M$ toutes les valuations qui vont suivre seront bien définies. On choisit des bases de $M$ et de $\Delta$. Ce sont donc aussi des bases de $N$ et de $D$. On va appliquer les lemmes 3.4 et 3.7 aux cordonnées d'un élément de $R^{-1}(\widetilde{N})$. En effet $R^{-1}(\widetilde{N})$ est formé

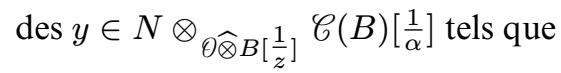




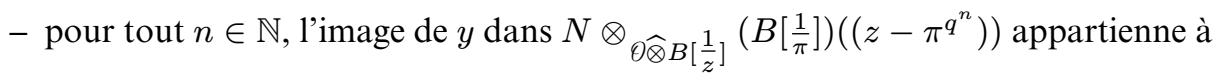

$$
\begin{aligned}
R^{-1}\left(\phi_{D} \cdots \tau^{n} \phi_{D} \tau^{n} V\right) & =R^{-1}\left(\phi_{D} \cdots \tau^{n-1} \phi_{D} \tau^{n} R\left(\tau^{n} N \otimes_{\vartheta \widehat{\otimes} B\left[\frac{1}{z}\right]}\left(B\left[\frac{1}{\pi}\right]\right)\left[\left[z-\pi^{q^{n}}\right]\right]\right)\right) \\
& =\phi_{N} \cdots \tau^{n-1} \phi_{N}\left(\tau^{n} N \otimes_{\vartheta \widehat{\otimes} B\left[\frac{1}{z}\right]}\left(B\left[\frac{1}{\pi}\right]\right)\left[\left[z-\pi^{q^{n}}\right]\right]\right) \\
& =N \otimes_{\oslash \widehat{\otimes} B\left[\frac{1}{z}\right]}\left(B\left[\frac{1}{\pi}\right]\right)\left[\left[z-\pi^{q^{n}}\right]\right]
\end{aligned}
$$

puisque $\phi_{N}, \ldots, \tau^{n-1} \phi_{N}$ n'ont pas de pôle en $z=\pi^{q^{n}}$. D'après le lemme 3.4 cette condition équivaut à $y \in N \otimes_{\oslash \widehat{\otimes} B\left[\frac{1}{z}\right]} \mathscr{C}(B)$.

- il existe $C \in \mathbb{R}$ telle que pour tout $n \in \mathbb{N}$,

$$
\nu_{q^{n}}\left(\left(\phi_{D} \cdots \tau^{n-1} \phi_{D}\right)^{-1} R y\right) \geq C q^{n} .
$$

Comme ${ }^{\tau^{n-1}} \phi_{D}^{-1} \cdots \phi_{D}^{-1} R y=\tau^{n} R^{\tau^{n-1}} \phi_{N}^{-1} \cdots \phi_{N}^{-1} y$ cette condition équivaut à l'existence de $C \in \mathbb{R}$ telle que, pour tout $n \in \mathbb{N}$,

$$
\nu_{q^{n}}\left(\tau^{n} R^{\tau^{n-1}} \phi_{N}^{-1} \cdots \phi_{N}^{-1} y\right) \geq C q^{n} .
$$

Il existe $C_{1} \in \mathbb{N}$ tel que les coefficients de $R$ et $R^{-1}$ appartiennent à $z^{-C_{1}} \oslash \widehat{\otimes}_{\theta_{L}}\left[\left[\frac{\pi}{z}\right]\right]$, d'où $\nu_{q^{n}}\left(\tau^{n} R\right) \geq-C_{1} q^{n}$ et $\nu_{q^{n}}\left(\tau^{n} R^{-1}\right) \geq-C_{1} q^{n}$ et donc

$$
\left|\nu_{q^{n}}\left(\tau^{n} R^{\tau^{n-1}} \phi_{N}^{-1} \cdots \phi_{N}^{-1} y\right)-\nu_{q^{n}}\left(\tau^{n-1} \phi_{N}^{-1} \cdots \phi_{N}^{-1} y\right)\right| \leq C_{1} q^{n} .
$$

Enfin la condition (PIL) et le lemme 3.5 donnent l'encadrement suivant :

$$
\begin{aligned}
-t\left(q^{n-1}+\cdots+1\right)-C_{0}+\nu_{q^{n}}(y) & \leq \nu_{q^{n}}\left(\tau^{n-1} \phi_{N}^{-1} \cdots \phi_{N}^{-1} y\right) \\
& \leq-s\left(q^{n-1}+\cdots+1\right)+C_{0}+\nu_{q^{n}}(y) .
\end{aligned}
$$

On voit alors que cette condition équivaut à l'existence de $C \in \mathbb{R}$ telle que, pour tout $n \in \mathbb{N}, \nu_{q^{n}}(y) \geq C q^{n}$.

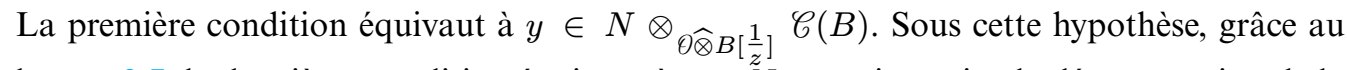
lemme 3.7, la deuxième condition équivaut à $y \in N$, ce qui termine la démonstration de la proposition 3.12 .

On peut exprimer la construction précédente en termes de catégories, en se plaçant de nouveau sur une base générale.

Définition 3.13. - a) Soient $S$ un schéma $\pi$-adique plat sur $\operatorname{Spf} \oslash \widehat{\otimes} k$ et $S_{0}$ le fermé de $S$ d'idéal $\pi$. On appelle pseudo-isochtouca local rigidifié modulo $\pi$ par un isochtouca local sur $k$ un triplet $\left(\left(D, \phi_{D}\right),\left(N, \phi_{N}\right), \rho\right)$ où $\left(N, \phi_{N}\right)$ est un pseudo-isochtouca local sur $S,\left(D, \phi_{D}\right)$ un isochtouca local sur $k$, et $\rho$ un isomorphisme entre la restriction de $\left(N, \phi_{N}\right) \grave{a} S_{0}$ et l'image inverse de $\left(D, \phi_{D}\right) \grave{a} S_{0}$. Les morphismes sont évidents.

b) Soit \& une variété rigide analytique sur $\mathrm{Spm} K$. La catégorie des isochtoucas locaux sur $k$ avec structure de Hodge-Pink définie sur \& est la catégorie des triplets $\left(D, \phi_{D}, V\right)$, où $\left(D, \phi_{D}\right)$ est un isochtouca local sur $k$, et $V$ est une structure de Hodge-Pink sur D définie sur \& (au sens de la définition 3.9). Un morphisme $\left(D, \phi_{D}, V\right) \rightarrow\left(D^{\prime}, \phi_{D^{\prime}}, V^{\prime}\right)$ dans cette catégorie est un morphisme $f:\left(D, \phi_{D}\right) \rightarrow\left(D^{\prime}, \phi_{D^{\prime}}\right)$ d'isochtoucas locaux sur $k$ tel que $\left({ }^{\tau} f \otimes 1\right)(V) \subset V^{\prime}$.

Dans cette définition, $\left(D, \phi_{D}\right)$ joue le rôle du $F$-isocristal de [51]. 
Proposition 3.14. - Soit $S$ un schéma $\pi$-adique plat sur $\operatorname{Spf} \Theta \widehat{\otimes} k$. Le foncteur

$$
\left(\left(D, \phi_{D}\right),\left(N, \phi_{N}\right), \rho\right) \mapsto\left(D, \phi_{D}, V\right)
$$

de la catégorie des pseudo-isochtoucas locaux sur $S$ rigidifiés modulo $\pi$ par un isochtouca local sur $k$ vers la catégorie des isochtoucas locaux sur $k$ avec structure de Hodge-Pink définie sur $S_{\text {rig }}$ est pleinement fidèle.

Démonstration. - Cela résulte immédiatement de la proposition 3.3, car c'est un énoncé local.

On note que la proposition 3.12 construit explicitement un quasi-inverse de ce foncteur sur son image essentielle. On appellera admissibles les objets de son image essentielle.

Proposition 3.15. - Soit $S$ un schéma $\pi$-adique plat sur $\operatorname{Spf} \theta \widehat{\otimes} k$. Une extension de deux isochtoucas locaux sur $k$ avec structure de Hodge-Pink (définie sur $S_{\text {rig }}$ ) admissibles est admissible.

Plus précisément, soient $\left(\left(D, \phi_{D}\right),\left(N, \phi_{N}\right), \rho\right)$ et $\left(\left(D^{\prime \prime}, \phi_{D^{\prime \prime}}\right),\left(N^{\prime \prime}, \phi_{N^{\prime \prime}}\right), \rho^{\prime \prime}\right)$ des pseudoisochtoucas locaux rigidifiés modulo $\pi$, et $\left(D, \phi_{D}, V\right)$ et $\left(D^{\prime \prime}, \phi_{D^{\prime \prime}}, V^{\prime \prime}\right)$ les isochtoucas locaux sur $k$ avec structure de Hodge-Pink qui leur sont associés. Alors toute extension de $\left(D^{\prime \prime}, \phi_{D^{\prime \prime}}, V^{\prime \prime}\right)$ par $\left(D, \phi_{D}, V\right)$ provient d'une extension de $\left(\left(D^{\prime \prime}, \phi_{D^{\prime \prime}}\right),\left(N^{\prime \prime}, \phi_{N^{\prime \prime}}\right), \rho^{\prime \prime}\right)$ par $\left(\left(D, \phi_{D}\right),\left(N, \phi_{N}\right), \rho\right)$.

$\operatorname{Si} \&$ est une variété rigide analytique sur $\operatorname{Spm} K$ et $\left(D, \phi_{D}\right),\left(D^{\prime}, \phi_{D^{\prime}}\right),\left(D^{\prime \prime}, \phi_{D^{\prime \prime}}\right)$ sont des isochtoucas locaux sur $k$ avec des structures de Hodge-Pink $V, V^{\prime}, V^{\prime \prime}$ définies sur $\&$, une suite exacte courte dans la catégorie des isochtoucas locaux sur $k$ avec structure de HodgePink est une suite exacte $0 \rightarrow D \rightarrow D^{\prime} \rightarrow D^{\prime \prime} \rightarrow 0$ dans la catégorie des isochtoucas locaux sur $k$ telle que $V^{\prime \prime}$ soit l'image de $V$ dans ${ }^{\tau} D^{\prime \prime} \otimes_{k((z))} \theta_{\phi}((z-\pi))$ et $V$ l'intersection de $V^{\prime}$ $\operatorname{avec}^{\tau} D \otimes_{k((z))} \theta_{\phi}((z-\pi))$.

Démonstration. - Grâce à la pleine fidélité du foncteur établie dans la proposition 3.14, on voit qu'il suffit de montrer l'énoncé dans le cas où $S$ est affine et où $N$ et $N^{\prime \prime}$ sont libres. On suppose donc que $S=\operatorname{Spf} B$ où $B$ est une $\theta \widehat{\otimes} k$-algèbre complète pour la topologie $\pi$-adique et sans $\pi$-torsion, et que $\left(N, \phi_{N}\right)$ et $\left(N^{\prime \prime}, \phi_{N^{\prime \prime}}\right)$ sont des pseudo-isochtoucas locaux libres sur $B$.

On se ramène à montrer la proposition 3.15 dans le cas où $\left(D^{\prime \prime}, \phi_{D^{\prime \prime}}, V_{D^{\prime \prime}}\right)$ est trivial (on indiquera dans une remarque comment le lecteur qui le souhaiterait peut traiter directement le cas général, au prix de notations plus compliquées). En effet la catégorie des pseudoisochtoucas locaux rigidifiés modulo $\pi$ et celle des isochtoucas locaux sur $k$ avec structure de Hodge-Pink possèdent des opérations produit tensoriel et dual. Par exemple (en oubliant les rigidifications pour simplifier les notations), le produit tensoriel de $\left(N, \phi_{N}\right)$ et du dual de $\left(N^{\prime \prime}, \phi_{N^{\prime \prime}}\right)$ est $\left(N \otimes N^{\prime \prime *}, \phi_{N} \otimes{ }^{t} \phi_{N^{\prime \prime}}^{-1}\right)$ et il est équivalent de se donner une extension de $\left(\theta \widehat{\otimes} B\left[\frac{1}{z}\right]\right.$, Id) par cet objet ou une extension de $\left(N, \phi_{N}\right) \operatorname{par}\left(N^{\prime \prime}, \phi_{N^{\prime \prime}}\right)$. On est donc ramené à montrer la proposition 3.15 dans le cas où $\left(D^{\prime \prime}, \phi_{D^{\prime \prime}}, V_{D^{\prime \prime}}\right)$ est trivial, c'est-à-dire

$$
D^{\prime \prime}=k((z)), \phi_{D^{\prime \prime}}=\mathrm{Id} \text {, et } V_{D^{\prime \prime}}=\left(B\left[\frac{1}{\pi}\right]\right)[[z-\pi]] .
$$

On doit montrer que $D^{\prime}$ est admissible. On a un pseudo-isochtouca local libre $\left(N, \phi_{N}\right)$ et un isomorphisme $\rho$ entre la restriction de $\left(N, \phi_{N}\right)$ à $B / \pi B$ et l'image inverse de $\left(D, \phi_{D}\right)$ à 
$B / \pi B$, tel que $V$ soit associé à $\left(N, \phi_{N}\right)$ et $\rho$, on se donne une extension $D^{\prime}=\left(D^{\prime}, \phi_{D^{\prime}}, V^{\prime}\right)$ de $D^{\prime \prime}$ par $D$ et on va trouver un pseudo-isochtouca local libre $\left(N^{\prime}, \phi_{N^{\prime}}\right)$ extension du pseudoisochtouca local trivial par $\left(N, \phi_{N}\right)$, et $\rho^{\prime}$ extension de $\rho$, tels que $V^{\prime}$ soit associé à $\left(N^{\prime}, \phi_{N^{\prime}}\right)$ et $\rho^{\prime}$.

On fixe un isomorphisme de $k((z))$-espaces vectoriels $D^{\prime}=D \oplus k((z))$. On a alors $\phi_{D^{\prime}}=\left(\begin{array}{cc}\phi_{D} & Y \\ 0 & 1\end{array}\right)$ pour un certain $Y \in D$ et $V^{\prime}$ est un $\left(B\left[\frac{1}{\pi}\right]\right)[[z-\pi]]$-réseau de ${ }^{\tau} D^{\prime} \otimes_{k((z))}\left(B\left[\frac{1}{\pi}\right]\right)((z-\pi))$ qui est une extension de $\left(B\left[\frac{1}{\pi}\right]\right)[[z-\pi]]$ par $V$. Il existe $T \in{ }^{\tau} D \otimes_{k((z))}\left(B\left[\frac{1}{\pi}\right]\right)((z-\pi))$ tel que

$$
(T, 1) \in{ }^{\tau} D \otimes_{k((z))}\left(B\left[\frac{1}{\pi}\right]\right)((z-\pi)) \oplus\left(B\left[\frac{1}{\pi}\right]\right)((z-\pi))={ }^{\tau} D^{\prime} \otimes_{k((z))}\left(B\left[\frac{1}{\pi}\right]\right)((z-\pi))
$$

appartienne à $V^{\prime}$ et $T$ est unique modulo $V$. En d'autres termes $V^{\prime}$ est déterminé par un élément $T \in{ }^{\tau} D \otimes_{k((z))}\left(B\left[\frac{1}{\pi}\right]\right)((z-\pi)) / V$.

On pose $N^{\prime}=N \oplus \theta \widehat{\otimes} B\left[\frac{1}{z}\right]$ comme $\theta \widehat{\otimes} B\left[\frac{1}{z}\right]$-module, avec $\rho^{\prime}=\left(\begin{array}{ll}\rho & 0 \\ 0 & 1\end{array}\right)$. Soit $X_{0} \in N\left[\frac{1}{z-\pi}\right]$ dont la réduction modulo $\pi$ est $\rho^{-1} Y$. On pose $\phi_{N^{\prime}}=\left(\begin{array}{cc}\phi_{N} X_{0}+X \\ 0 & 1\end{array}\right)$, avec $X \in \pi N\left[\frac{1}{z-\pi}\right]$ arbitraire (nous choisirons ensuite $X$ tel que la structure de Hodge-Pink associée à $\phi_{N^{\prime}}$ soit $\left.V^{\prime}\right)$.

Lemme 3.16. - Avec les notations précédentes $\left(N^{\prime}, \phi_{N^{\prime}}\right)$ est un pseudo-isochtouca local.

Démonstration. - La condition (PIL) de la définition 2.1 est vérifiée car dans le produit $\phi_{N^{\prime}}{ }^{\tau} \phi_{N^{\prime}} \ldots \tau^{n-1} \phi_{N^{\prime}}$ le terme non diagonal apparaît 0 ou 1 fois, donc n'ajoute pas de dénominateur en $z$ d'ordre plus grand qu'une constante indépendante de $n$, et il en va de même pour $\tau^{n-1} \phi_{N^{\prime}}^{-1} \ldots{ }^{\tau} \phi_{N^{\prime}}^{-1} \phi_{N^{\prime}}^{-1}$

On va montrer qu'on peut choisir $X$ de sorte que si

$$
R^{\prime}: N^{\prime} \otimes_{\partial \widehat{\otimes} B\left[\frac{1}{z}\right]} \mathscr{C}(B)\left[\frac{1}{\alpha}\right] \rightarrow D^{\prime} \otimes_{k((z))} \mathscr{C}(B)\left[\frac{1}{\alpha}\right]
$$

est l'unique morphisme congru à $\rho^{\prime}$ modulo $\pi$ vérifiant

$$
R^{\prime}\left(\phi_{N^{\prime}} \otimes 1\right)=\left(\phi_{D^{\prime}} \otimes 1\right)^{\tau} R^{\prime}
$$

alors

$$
\begin{aligned}
& { }^{\tau} R^{\prime}\left(\phi_{N^{\prime}}^{-1}\left(N^{\prime} \otimes_{\oslash \widehat{\otimes} B\left[\frac{1}{z}\right]}\left(B\left[\frac{1}{\pi}\right]\right)[[z-\pi]]\right)\right)=\phi_{D^{\prime}}^{-1}\left(R^{\prime}\left(N^{\prime} \otimes_{\oslash \widehat{\otimes} B\left[\frac{1}{z}\right]}\left(B\left[\frac{1}{\pi}\right]\right)[[z-\pi]]\right)\right) \\
& \subset^{\tau} D^{\prime} \otimes_{k((z))}\left(B\left[\frac{1}{\pi}\right]\right)((z-\pi))
\end{aligned}
$$

soit l'extension $V^{\prime}$ de $\left(B\left[\frac{1}{\pi}\right]\right)[[z-\pi]]$ par $V$ qui est donnée au départ (et qui est une extension absolument arbitraire). On a $R^{\prime}=\left(\begin{array}{ll}R & Z \\ 0 & 1\end{array}\right)$ pour un certain

$$
Z \in D \otimes_{k((z))} \mathscr{C}(B)\left[\frac{1}{\alpha}\right] \subset D \otimes_{k((z))}\left(B\left[\frac{1}{\pi}\right]\right)((z-\pi))
$$


dépendant de $X$ et il s'agit de montrer que l'on peut trouver $X$ tel que $\phi_{D}^{-1}(Z) \in{ }^{\tau} D \otimes_{k((z))}$ $\left(B\left[\frac{1}{\pi}\right]\right)((z-\pi))$ soit égal modulo $V$ à

$$
T \in{ }^{\tau} D \otimes_{k((z))}\left(B\left[\frac{1}{\pi}\right]\right)((z-\pi)) / V .
$$

Cela est équivalent à la condition suivante :

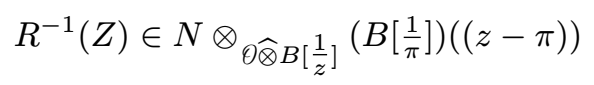

est égal modulo $N \otimes_{\widehat{\partial} \widehat{\otimes}\left[\frac{1}{z}\right]}\left(B\left[\frac{1}{\pi}\right]\right)[[z-\pi]]$ à

$$
\phi_{N}\left({ }^{\tau} R^{-1}(T)\right)=R^{-1}\left(\phi_{D}(T)\right) \in N \otimes_{\widehat{\partial} \widehat{\otimes} B\left[\frac{1}{z}\right]}\left(\left(B\left[\frac{1}{\pi}\right]\right)((z-\pi)) /\left(B\left[\frac{1}{\pi}\right]\right)[[z-\pi]]\right) .
$$

Calculons $R^{-1}(Z)$ en fonction de $X$. Comme $R^{\prime}=\left(\begin{array}{ll}R & Z \\ 0 & 1\end{array}\right)$ vérifie

$$
R^{\prime}\left(\begin{array}{cc}
\phi_{N} & X_{0}+X \\
0 & 1
\end{array}\right)=\left(\begin{array}{cc}
\phi_{D} & Y \\
0 & 1
\end{array}\right){ }^{\tau} R^{\prime}
$$

on obtient l'équation $Z=\left(Y-R X_{0}-R X\right)+\phi_{D}^{\tau} Z$, d'où

$$
R^{-1}(Z)=R^{-1}(Y)-X_{0}-X+\phi_{N}{ }^{\tau}\left(R^{-1}(Z)\right) \text {. }
$$

On a donc

$$
R^{-1}(Z)=R^{-1}\left(Z_{0}\right)-\left(X+\phi_{N}{ }^{\tau} X+\phi_{N}{ }^{\tau} \phi_{N} \tau^{2} X+\cdots\right)
$$

où $Z_{0}$ correspond à $X=0$ et où la somme dans le membre de droite converge au sens suivant : si $C$ est tel que $\phi_{N} \in(z-\pi)^{-C} \operatorname{Hom}\left({ }^{\tau} N, N\right)$ et $X \in \frac{\pi}{(z-\pi)^{C}} N$, la série $\alpha^{C}\left(X+\phi_{N}{ }^{\tau} X+\phi_{N}{ }^{\tau} \phi_{N} \tau^{2} X+\cdots\right)$ converge dans $N \otimes_{\widehat{\partial} \widehat{\otimes} B\left[\frac{1}{z}\right]} \mathscr{C}(B)$. Grâce au lemme 3.2 on est donc ramené à montrer le lemme suivant (avec $\phi$ égal à la matrice de $\phi_{N}$ dans une base de $N$ ).

Lemme 3.17. - Soient $C \in \mathbb{N}^{*}$ et $\phi \in z^{-C}(z-\pi)^{-C} M_{r}(\Theta \widehat{\otimes} B)$. Alors l'application

$$
\theta:\left(\frac{\pi}{(z-\pi)^{C}} \theta \widehat{\otimes} B\left[\frac{1}{z}\right]\right)^{r} \rightarrow \frac{1}{(z-\pi)^{C}}\left(B\left[\frac{1}{\pi}\right]\right)[[z-\pi]]^{r} /\left(B\left[\frac{1}{\pi}\right]\right)[[z-\pi]]^{r}
$$

qui à $X$ associe $X+\phi^{\tau} X+\phi^{\tau} \phi^{\tau^{2}} X+\cdots$ est surjective.

Démonstration. - L'espace d'arrivée $\frac{1}{(z-\pi)^{C}}\left(B\left[\frac{1}{\pi}\right]\right)[[z-\pi]]^{r} /\left(B\left[\frac{1}{\pi}\right]\right)[[z-\pi]]^{r}$ est un $B\left[\frac{1}{\pi}\right]$-module libre de dimension $C r$ muni de la topologie $\pi$-adique (comme dans le lemme 3.2). L'application

$$
\theta_{0}:\left(\frac{\pi}{(z-\pi)^{C}} \Theta \widehat{\otimes} B\left[\frac{1}{z}\right]\right)^{r} \rightarrow \frac{1}{(z-\pi)^{C}}\left(B\left[\frac{1}{\pi}\right]\right)[[z-\pi]]^{r} /\left(B\left[\frac{1}{\pi}\right]\right)[[z-\pi]]^{r}
$$

qui à $X$ associe $X$ est clairement surjective. D'autre part pour tout $X \in\left(\frac{\pi}{(z-\pi)^{C}} \ominus \widehat{\otimes} B\left[\frac{1}{z}\right]\right)^{r}$ la suite $\theta\left(\left(\frac{\pi}{z}\right)^{q^{k}} X\right)$ tend vers $\theta_{0}(X)$, pour la topologie $\pi$-adique, quand $k$ tend vers l'infini. En effet si $q^{k} \geq C$ on a $\theta\left(\left(\frac{\pi}{z}\right)^{q^{k}} X\right)=X+\pi^{q^{k+1}-q^{k}} \phi^{\tau} X+$ $\pi^{q^{k+2}-q^{k}} \phi^{\tau} \phi^{\tau^{2}} X+\cdots$ Donc l'image de $\theta$ est dense. 
Pour tout $k>C^{2}$, on a

$$
\begin{gathered}
\theta_{0}\left(\left(\frac{\pi^{k}}{(z-\pi)^{C}} \Theta \widehat{\otimes} B\right)^{r}\right)=\pi^{k}\left(\frac{1}{(z-\pi)^{C}} B[[z-\pi]]^{r} / B[[z-\pi]]^{r}\right) \\
\text { et }\left(\theta-\theta_{0}\right)\left(\left(\frac{\pi^{k}}{(z-\pi)^{C}} \Theta \widehat{\otimes} B\right)^{r}\right) \subset \pi^{q k-2 C^{2}}\left(\frac{1}{(z-\pi)^{C}} B[[z-\pi]]^{r} / B[[z-\pi]]^{r}\right) .
\end{gathered}
$$

Pour la dernière inclusion on utilise le fait que $\frac{\pi^{C}}{z}, \frac{\pi^{C}}{z-\pi^{q}}, \frac{\pi^{C}}{z-\pi^{q^{2}}}, \ldots$ appartiennent à $B[[z-\pi]] /(z-\pi)^{C} B[[z-\pi]] \subset\left(B\left[\frac{1}{\pi}\right]\right)[[z-\pi]] /(z-\pi)^{C}\left(B\left[\frac{1}{\pi}\right]\right)[[z-\pi]]$ ainsi que l'égalité $q k-2 C^{2}=\inf _{i \in \mathbb{N}^{*}} q^{i} k-2 i C^{2}$. Donc l'image de $\theta$ contient un voisinage de 0 . Ceci achève la démonstration du lemme 3.17 et donc celle de la proposition 3.15.

Remarque. - On peut adapter la démonstration précédente au cas général où l'on ne suppose pas $\left(D^{\prime \prime}, \phi_{D^{\prime \prime}}, V_{D^{\prime \prime}}\right)$ trivial, en prenant $Y \in D \otimes D^{\prime \prime *}, X \in \pi N \otimes N^{\prime \prime *}\left[\frac{1}{z-\pi}\right]$, et en appliquant le lemme 3.17 à la matrice de $\phi_{N} \otimes{ }^{t} \phi_{N^{\prime \prime}}^{-1}$ dans une base de $N \otimes N^{\prime \prime *}$.

\section{Puissances divisées adaptées à une théorie entière}

Soit $B$ une $\theta$-algèbre complète pour la topologie $\pi$-adique et sans $\pi$-torsion. Soit $m \in \mathbb{N}^{*}$. Considérons la catégorie des chtoucas locaux $\left(M, \phi_{M}\right)$ d'amplitude $\subset[0, m]$, c'est-à-dire tels que $\phi_{M}$ et $(z-\pi)^{m} \phi_{M}^{-1}$ n'aient pas de dénominateurs en $z-\pi: m=1$ correspond au cas minuscule. Dans les paragraphes 5, 6 et 10 nous construirons un début de théorie entière pour les chtoucas locaux minuscules. La proposition suivante indique quelle condition de puissances divisées on doit imposer à un idéal $I$ de $B$ pour que $M$ soit déterminé par sa classe d'isogénie et sa réduction modulo $I$ (cela est nécessaire pour l'existence d'une théorie entière relativement à ces puissances divisées). Le lecteur constatera que pour $m>1$ le résultat n'est pas bon, et notamment moins bon qu'en inégales caractéristiques, c'est pourquoi nous nous restreignons dans les paragraphes 5, 6 et 10 aux chtoucas locaux minuscules. Dans le paragraphe 11 nous retrouverons une situation comparable à celle d'inégales caractéristiques en nous restreignant aux chtoucas locaux tranquilles (nous verrons aussi que les chtoucas locaux minuscules sont tranquilles).

Soient $\left(M, \phi_{M}\right)$ et $\left(M^{\prime}, \phi_{M^{\prime}}\right)$ des chtoucas locaux d'amplitude $\subset[0, m]$. Dans la suite, si $I$ est un idéal fermé de $B$ et $k \in \mathbb{N}^{*}$, on note $I^{(k)}$ l'idéal fermé de $B$ engendré par les $x^{k}$ pour $x \in I$.

Proposition 4.1. - Soit I un idéal fermé de B tel que $\frac{I^{(q-1)}}{\pi^{m}}$ soit inclus dans $B \subset B\left[\frac{1}{\pi}\right]$ et soit formé d'éléments topologiquement nilpotents de B. Si f est un morphisme de chtoucas locaux de $\left(M, \phi_{M}\right)$ dans $\left(M^{\prime}, \phi_{M^{\prime}}\right)$, tel que $f$ modulo I est divisible par $z$, alors $f$ est divisible par $z$.

Autrement dit la connaissance de $M$ à isogénie près et de $M / I$ détermine $M$.

Démonstration. - Soient $\bar{M}=M / z M, \overline{M^{\prime}}=M^{\prime} / z M^{\prime}$, et $\overline{\phi_{M}}, \overline{\phi_{M^{\prime}}}, \overline{(z-\pi)^{m} \phi_{M}^{-1}}$, $\overline{(z-\pi)^{m} \phi_{M^{\prime}}^{-1}}, \bar{f}$ et $\bar{\tau} f$ les réductions de $\phi_{M}, \phi_{M^{\prime}},(z-\pi)^{m} \phi_{M}^{-1},(z-\pi)^{m} \phi_{M^{\prime}}^{-1}, f$ et ${ }^{\tau} f$ modulo $z$. On a $(-\pi)^{m} \bar{f}=\overline{\phi_{M^{\prime}}}{ }^{\tau} \bar{f} \circ \overline{(z-\pi)^{m} \phi_{M}^{-1}}$. Comme $\bar{f}$ est nul modulo un idéal de type fini $J \subset I$, $\bar{\tau} f$ est nul modulo $J^{(q)}$ et $\bar{f}$ est nul modulo $\frac{J^{(q)}}{\pi^{m}}$. En itérant on voit que pour tout $k \in \mathbb{N}, \bar{f}$ 
est nul modulo $\frac{J^{\left(q^{k}\right)}}{\pi^{m\left(1+q+\cdots+q^{k-1}\right)}} \subset\left(\frac{J^{(q-1)}}{\pi^{m}}\right)^{\left(1+q+\cdots+q^{k-1}\right)}$. Comme $B$ est séparé, $\bar{f}=0$ et la proposition est démontrée.

Voici maintenant un contre-exemple montrant que dans la proposition 4.1 l'hypothèse de nilpotence topologique des puissances divisées est nécessaire. Supposons que $B$ contient $\zeta$ tel que $\zeta^{q-1}=(-\pi)^{m}$. Alors

$$
\left(\begin{array}{ll}
1 & \frac{\zeta}{z} \\
0 & 1
\end{array}\right)\left(\begin{array}{cc}
1 & 0 \\
0 & (z-\pi)^{m}
\end{array}\right)\left(\begin{array}{cc}
1-\frac{\zeta^{q}}{z} \\
0 & 1
\end{array}\right)=\left(\begin{array}{cc}
1 & \frac{\zeta(z-\pi)^{m}-\zeta^{q}}{z} \\
0 & (z-\pi)^{m}
\end{array}\right)
$$

et cette dernière matrice n'a pas de dénominateur en $z$. Posons

$$
\begin{aligned}
M & =(\Theta \widehat{\otimes} B)^{2} \text { muni de } \phi_{M}=\left(\begin{array}{cc}
1 & 0 \\
0 & (z-\pi)^{m}
\end{array}\right) \\
\text { et } M^{\prime} & =(\Theta \widehat{\otimes} B)^{2} \text { muni de } \phi_{M^{\prime}}=\left(\begin{array}{cc}
1 & \frac{\zeta(z-\pi)^{m}-\zeta^{q}}{z} \\
0 & (z-\pi)^{m}
\end{array}\right) .
\end{aligned}
$$

Alors $f=z\left(\begin{array}{ll}1 & \zeta \\ 0 & 1\end{array}\right)$ est un morphisme de $\left(M, \phi_{M}\right)$ vers $\left(M^{\prime}, \phi_{M^{\prime}}\right)$ qui n'est pas divisible par $z$ alors que sa réduction modulo $I=(\zeta)$ est divisible par $z$.

\section{Cristaux associés aux chtoucas locaux minuscules}

Dans ce paragraphe, nous considérons des puissances divisées au sens de Honda et de Gross-Hopkins [33]. Nous montrons comment associer un « $\theta$-cristal» (au sens de Honda et de Gross-Hopkins) à un chtouca local minuscule sur une base affine $\pi$-adique (pouvant contenir de la $\pi$-torsion).

\subsection{Puissances divisées et $\vartheta$-cristaux au sens de Honda et de Gross-Hopkins}

Définition 5.1. - Soient B une Ө-algèbre et I un idéal de B. Une structure de puissances divisées au sens de Honda et de Gross-Hopkins sur I est un triplet $(\hat{B}, \beta, \gamma)$ où

- $\hat{B}$ est une $\theta$-algèbre,

- $\beta: B \rightarrow \hat{B}$ est un morphisme de $\theta$-algèbres,

- et $\gamma$ est une application de I dans $\hat{B}$, qui vérifie
(a) $\pi \gamma(x)=\beta(x)^{q}$ pour $x \in I$,
(b) $\gamma(b x)=\beta(b)^{q} \gamma(x)$ pour $x \in I$ et $b \in B$,
(c) $\gamma(x+y)=\gamma(x)+\gamma(y)$ pour $x, y \in I$.

Remarque. - Dans la définition précédente, les conditions (b) et (c) résultent de (a) si $\hat{B}$ est sans $\pi$-torsion. Cependant on n'impose pas à $\gamma$ toutes les relations qui résulteraient de (a) si $\hat{B}$ était sans $\pi$-torsion. Dans l'exemple 5.7 ci-dessous on verra que pour $k \in \mathbb{N}^{*}$, et $x_{1}, \ldots, x_{k}, y_{1}, \ldots, y_{k} \in I$ tels que $\sum_{i=1}^{k} x_{i} y_{i}=0$, on n'a pas nécessairement $\sum_{i=1}^{k} \gamma\left(x_{i}\right) \gamma\left(y_{i}\right)=0$ dans $\hat{B}$ et que, si $\pi$ appartient à $I$, on n'a pas nécessairement $\gamma(\pi)=\pi^{q-1}$. 
Le propre des puissances divisées de Honda et Gross-Hopkins est que l'on ne compose pas $\gamma$ avec lui-même. C'est pourquoi $\gamma$ ne prend pas ses valeurs dans $I$ ni même dans $B$ mais dans une autre algèbre $\hat{B}$. Les puissances divisées de Honda et Gross-Hopkins sont adaptées à la construction des cristaux associés aux groupes $p$-divisibles, et dans notre cas d'égales caractéristiques à la construction des $\theta$-cristaux associés aux chtoucas locaux minuscules (qui sont le $\theta$-analogue des groupes $p$-divisibles). Au contraire les puissances divisées de Grothendieck et Berthelot [4, 6, 7, 8, 9] sont adaptées aux amplitudes plus grandes que $[0,1]$ en inégales caractéristiques. Cependant leur $\theta$-analogue n'est pas adapté aux chtoucas locaux d'amplitude plus grande que $[0,1]$ arbitraires, comme nous l'avons vu dans le paragraphe précédent, mais seulement aux chtoucas locaux tranquilles, comme nous le verrons dans le paragraphe 11. Les puissances divisées de Grothendieck et Berthelot (nilpotentes) sont également utiles pour le théorème de relèvement de Grothendieck, comme nous le verrons au paragraphe suivant. On renvoie au paragraphe suivant pour une définition précise du $\Theta$-analogue des puissances divisées de Grothendieck et Berthelot, et pour le lien logique avec les puissances divisées de Honda et Gross-Hopkins considérées dans ce paragraphe.

À l'instar de Katz [36] nous définissons un $\theta$-cristal par ses valeurs sur les $A$-objets test. Dans tout ce paragraphe, $A$ désigne une $\theta$-algèbre complète pour la topologie $\pi$-adique.

Définition 5.2. - On appelle A-objet test un sextuplet $(B, I, s, \hat{B}, \beta, \gamma)$ où

- B est une O-algèbre dans laquelle l'image de $\pi$ est nilpotente, I un idéal de $B$, et $s: A \rightarrow B / I$ un morphisme de O-algèbres,

- $(\hat{B}, \beta, \gamma)$ est une structure de puissances divisées au sens de Honda et de Gross-Hopkins sur $I$,

- il existe $n$ tel que, pour tout $x \in I, \gamma(x)^{q^{n}}=0$ dans $\hat{B}$.

Un morphisme d'un $A$-objet test $(B, I, s, \hat{B}, \beta, \gamma)$ vers un autre $A$-objet test $\left(B^{\prime}, I^{\prime}, s^{\prime}, \hat{B}^{\prime}, \beta^{\prime}, \gamma^{\prime}\right)$ est la donnée de morphismes de $\theta$-algèbres $f: B \rightarrow B^{\prime}$ et $\hat{f}: \hat{B} \rightarrow \hat{B}^{\prime}$ tels que $f(I) \subset I^{\prime}, \beta^{\prime} \circ f=\hat{f} \circ \beta, \gamma^{\prime} \circ f=\hat{f} \circ \gamma$ et $f \circ s=s^{\prime}$ (en notant encore $f$ l'application quotient de $B / I$ dans $B^{\prime} / I^{\prime}$ ).

Remarque. - Si l'image de $\pi$ dans $A$ est nilpotente, $(A, 0, \mathrm{Id}, A, \mathrm{Id}, 0)$ est un $A$-objet test.

Définition 5.3. - Un O-cristal $E$ sur $A$ est la donnée pour tout A-objet test $(B, I, s, \hat{B}, \beta, \gamma)$ d'un $\hat{B}$-module localement libre $E(B, I, s, \hat{B}, \beta, \gamma)$, et pour tout morphisme $f$ d'un $A$-objet test $(B, I, s, \hat{B}, \beta, \gamma)$ vers un $A$-objet test $\left(B^{\prime}, I^{\prime}, s^{\prime}, \hat{B}^{\prime}, \beta^{\prime}, \gamma^{\prime}\right)$ d'un isomorphisme de $\hat{B}^{\prime}$-modules

$$
E(B, I, s, \hat{B}, \beta, \gamma) \otimes_{\hat{B}} \hat{B}^{\prime} \simeq E\left(B^{\prime}, I^{\prime}, s^{\prime}, \hat{B}^{\prime}, \beta^{\prime}, \gamma^{\prime}\right),
$$

ces isomorphismes devant être compatibles à la composition.

Un morphisme $u: E \rightarrow E^{\prime}$ entre deux $\theta$-cristaux sur $A$ est la donnée pour tout $A$-objet test $(B, I, s, \hat{B}, \beta, \gamma)$ d'un morphisme de $\hat{B}$-modules

$$
u(B, I, s, \hat{B}, \beta, \gamma): E(B, I, s, \hat{B}, \beta, \gamma) \rightarrow E^{\prime}(B, I, s, \hat{B}, \beta, \gamma),
$$

fonctoriel relativement aux morphismes de $A$-objets test.

4 e SÉRIE - TOME $44-2011$ - No 2 
Si $\varphi: \operatorname{Spf} A^{\prime} \rightarrow \operatorname{Spf} A$ est associé à un morphisme $\varphi^{*}: A \rightarrow A^{\prime}$ de $\theta$-algèbres complètes pour la topologie $\pi$-adique, et $E$ est un $\theta$-cristal sur $A$ on note $\varphi^{*}(E)$ le $\theta$-cristal sur $A^{\prime}$ défini de la façon suivante : pour tout $A^{\prime}$-objet test $(B, I, s, \hat{B}, \beta, \gamma)$,

$$
\varphi^{*}(E)(B, I, s, \hat{B}, \beta, \gamma)=E\left(B, I, s \circ \varphi^{*}, \hat{B}, \beta, \gamma\right) .
$$

La catégorie des $\theta$-cristaux sur $A$ est $\theta$-linéaire. Une isogénie est un morphisme $u: E \rightarrow E^{\prime}$ tel qu'il existe $v: E^{\prime} \rightarrow E$ et $e: \operatorname{Spf} A \rightarrow \mathbb{N}$ une fonction localement constante tels que $u \circ v=z^{e}$ et $v \circ u=z^{e}$.

Si $\pi=0$ dans $A$, on possède le morphisme de Frobenius $\varphi: \operatorname{Spf} A \rightarrow \operatorname{Spf} A$ (défini $\operatorname{par} \varphi^{*}(x)=x^{q}$ pour $x \in A$ ) et on appelle $F$-cristal un $\theta$-cristal $E$ muni d'une isogénie $\varphi^{*}(E) \rightarrow E$.

Nous allons définir une variante de la notion de $\theta$-cristal en imposant aux puissances divisées une condition de compatibilité. L'idéal $\pi \theta$ de $\theta$ est muni d'une structure de puissances divisées $\gamma_{\ominus}$ (au sens de la définition 5.1) définie par $\gamma_{\Theta}(\pi)=\pi^{q-1}$.

Définition 5.4. - Soient B une Ө-algèbre et I un idéal de B. Une structure de puissances divisées $(\hat{B}, \beta, \gamma)$ sur I est dite compatible à $\left(\theta, \pi \vartheta, \gamma_{\vartheta}\right)$ si $\gamma$ s'étend en $\gamma^{\prime}: I+\pi B \rightarrow \hat{B}$ tel que $\gamma^{\prime}(\pi)=\pi^{q-1}$ et que $\left(\hat{B}, \beta, \gamma^{\prime}\right)$ soit une structure de puissances divisées sur $I+\pi B$.

Un $A$-objet test relativement à $\left(\theta, \pi \vartheta, \gamma_{\vartheta}\right)$ est un $A$-objet test $(B, I, s, \hat{B}, \beta, \gamma)$ tel que $(\hat{B}, \beta, \gamma)$ soit compatible à $\left(\theta, \pi \theta, \gamma_{\vartheta}\right)$.

On définit un $\theta$-cristal relativement à $\left(\theta, \pi \theta, \gamma_{\theta}\right)$ de la même façon qu'un $\theta$-cristal mais en se limitant aux $A$-objets test relativement à $\left(\theta, \pi \theta, \gamma_{\theta}\right)$. En toute rigueur les $\theta$-cristaux au sens de la définition 5.3 étaient relatifs à $(\theta, 0,0)$.

Lemme 5.5. - L'image inverse par le morphisme $\operatorname{Spec} A / \pi A \rightarrow \operatorname{Spf} A$ fournit une équivalence de la catégorie des $\theta$-cristaux relativement à $\left(\theta, \pi \theta, \gamma_{\Theta}\right)$ sur $A$ vers la catégorie des $\theta$-cristaux relativement à $\left(\theta, \pi \theta, \gamma_{\theta}\right)$ sur $A / \pi A$.

Remarque. - La notion de $\theta$-cristal relativement à $\left(\theta, \pi \theta, \gamma_{\theta}\right)$ que nous venons de définir ne servira pas dans ce paragraphe, mais seulement dans le paragraphe 10. Par ailleurs nous y évaluerons un $\theta$-cristal relativement à $\left(\theta, \pi \theta, \gamma_{\vartheta}\right)$ en un pro-objet test, c'est-à-dire un système projectif d'objets test.

\subsection{Construction du foncteur de Dieudonné}

Nous allons construire un foncteur $\mathbb{D}$ (pour Dieudonné) de la catégorie des chtoucas locaux minuscules sur $A$ vers celle des $\theta$-cristaux sur $A$, qui vérifiera la proposition suivante.

Proposition 5.6. - Le foncteur $\mathbb{D}$ est compatible au changement de base et tel que, si l'image de $\pi$ dans $A$ est nilpotente, pour tout chtouca local minuscule $\left(M, \phi_{M}\right)$ sur $A$, la valeur de $\mathbb{D}\left(M, \phi_{M}\right)$ en le $A$-objet test $(A, 0, \operatorname{Id}, A, \operatorname{Id}, 0)$ soit ${ }^{\tau} M /(z-\pi)^{\tau} M$. Si $\pi=0$ dans $A$, $\mathbb{D}$ se relève en un foncteur de la catégorie des chtoucas locaux minuscules sur $A$ vers celle des F-cristaux sur $A$. 
Le plan pour la construction de $\mathbb{D}$ est le suivant. $\operatorname{Si}(B, I, s, \hat{B}, \beta, \gamma)$ est un $A$-objet test et $n \in \mathbb{N}^{*}$ est tel que

$$
\pi^{(q-1) n}=0 \text { dans } \hat{B} \text { et } \gamma(x)^{q^{n}}=0 \text { dans } \hat{B} \text { pour tout } x \in I
$$

nous construirons une $\theta \widehat{\otimes} B$-algèbre $\mathscr{D}_{n}(B, I)$ et un morphisme de $\theta \widehat{\otimes} B$-algèbres $\delta:{ }^{\tau} \mathscr{D}_{n}(B, I) \rightarrow \hat{B}$, puis un foncteur $\mathcal{F}$ de la catégorie des chtoucas locaux minuscules sur $B / I$ vers la catégorie des $\mathscr{D}_{n}(B, I)$-modules qui sont libres localement pour la topologie de Zariski sur Spec $B$, et le foncteur $\mathbb{D}$ sera alors défini en posant que pour tout chtouca local minuscule $\left(M, \phi_{M}\right)$ sur $A$, la valeur de $\mathbb{D}\left(M, \phi_{M}\right)$ en le $A$-objet test $(B, I, s, \hat{B}, \beta, \gamma)$ est égale à

$$
\tau\left(\mathcal{F}\left(\left(M, \phi_{M}\right)_{B / I}\right)\right) \otimes_{\tau \mathscr{D}_{n}(B, I)} \hat{B}
$$

où $\left(M, \phi_{M}\right)_{B / I}=\left(M \otimes_{\vartheta \widehat{\otimes} A} \oslash \widehat{\otimes}(B / I), \phi_{M} \otimes 1\right)$ est le changement de base de $\left(M, \phi_{M}\right)$ de $A$ à $B / I$, qui est un chtouca local sur $B / I$.

La construction de $\mathscr{D}_{n}(B, I)$ et du foncteur $\mathcal{F}$ a lieu dans un cadre plus général. Soit $B$ une $\theta$-algèbre où l'image de $\pi$ est nilpotente et $I$ un idéal de $B$. Soit $n \in \mathbb{N}^{*}$. On note alors $\mathscr{D}_{n}(B, I)$ la $\Theta \widehat{\otimes} B$-algèbre engendrée par des générateurs $t$ et $\epsilon(x)$ pour $x \in I$, assujettis aux relations $z t=\pi, t^{n}=0, z \epsilon(x)=x, \epsilon(x)^{q^{n}}=0, \epsilon(b x)=b \epsilon(x)$, et $\epsilon(x+y)=\epsilon(x)+\epsilon(y)$ pour $b \in B$ et $x, y \in I$ (dans ces relations on a utilisé l'inclusion $B \subset \vartheta \widehat{\otimes} B$ qui envoie $x$ sur $1 \otimes x)$. Dans la suite on notera toujours $\frac{\pi}{z}$ au lieu de $t \in \mathscr{D}_{n}(B, I)$ et, pour $n \in \mathbb{N}^{*}, \frac{\pi^{n}}{z}$ au lieu de $\pi^{n-1} t$. On note $\mathscr{J}_{n}$ l'idéal de $\mathscr{D}_{n}(B, I)$ engendré par $\Theta \widehat{\otimes} I$ et par les $\epsilon(x)$ pour $x \in I$. On a un morphisme $\Theta \widehat{\otimes}(B / I) \rightarrow \mathscr{D}_{n}(B, I) / \mathscr{J}_{n}$.

La construction du foncteur $\mathcal{F}$ se ramènera à une construction locale pour la topologie de Zariski sur Spec $B$. Le recollement pour les $\mathscr{D}_{n}(B, I)$-modules provient du recollement pour les $\theta \widehat{\otimes} B$-modules et du fait que pour tout $f \in B \backslash\{0\}$, on a un isomorphisme naturel

$$
\mathscr{D}_{n}\left(B\left[f^{-1}\right], I\left[f^{-1}\right]\right)=\mathscr{D}_{n}(B, I) \otimes_{\oslash \widehat{\otimes} B} \oslash \widehat{\otimes}\left(B\left[f^{-1}\right]\right) .
$$

Remarque. - Si $\pi \in I, \epsilon(\pi)$ et $\frac{\pi}{z}$ ne sont pas nécessairement égaux dans $\mathscr{D}_{n}(B, I)$. De même pour $k \in \mathbb{N}^{*}$ et $x_{1}, \ldots, x_{k}, y_{1}, \ldots, y_{k} \in I$ vérifiant $\sum_{i=1}^{k} x_{i} y_{i}=0, \sum_{i=1}^{k} \epsilon\left(x_{i}\right) \epsilon\left(y_{i}\right)$ n'est pas nécessairement nul dans $\mathscr{D}_{n}(B, I)$. On renvoie à l'exemple 5.7 ci-dessous pour une situation où cela se produit.

On rappelle les notations

$$
{ }^{\tau} \mathscr{D}_{n}(B, I)=\mathscr{D}_{n}(B, I) \otimes_{\vartheta \widehat{\otimes} B, 1 \widehat{\otimes} \mathrm{Fr}} \oslash \widehat{\otimes} B
$$

et ${ }^{\tau} x=x \otimes 1$ pour $x \in \mathscr{D}_{n}(B, I)$. Soit $(\hat{B}, \beta, \gamma)$ une structure de puissances divisées au sens de Honda et de Gross-Hopkins vérifiant la condition (5). On munit $\hat{B}$ d'une structure de $\theta \widehat{\otimes} B$-algèbre grâce au morphisme $\theta \widehat{\otimes} B \rightarrow \hat{B}$ donné par $a \widehat{\otimes} b \mapsto a \beta(b)$. On note que l'image de $z-\pi$ par ce morphisme est nulle. On définit un morphisme de $\widehat{\theta} \widehat{\otimes} B$-algèbres $\delta:{ }^{\tau} \mathscr{D}_{n}(B, I) \rightarrow \hat{B}$ en posant $\delta\left({ }^{\tau}(a \otimes b)\right)=a \beta(b)^{q}$ pour $a \in \emptyset$ et $b \in B, \delta\left({ }^{\tau}\left(\frac{\pi}{z}\right)\right)=\pi^{q-1}$ et $\delta(\tau(\epsilon(x)))=\gamma(x)$ pour $x \in I$.

Voici maintenant l'exemple annoncé. 
Exemple 5.7. - Soient $q \geq 3, B=\mathbb{F}_{q}[\pi, x] /\left(\pi^{q}, x^{q}, \pi x\right), I=(x, \pi), A=\mathbb{F}_{q}, s=\mathrm{Id}$, $\hat{B}=B, \beta=\mathrm{Id}$, et $\gamma: I \rightarrow \hat{B}$ nul sur $I^{2}=\left(x^{2}, \pi^{2}\right)$ et déterminé par $\gamma(x)=x$ et $\gamma(\pi)=x$. On voit que $\gamma(\pi) \neq \pi^{q-1}$ et $\gamma(\pi) \gamma(x) \neq 0$ alors que $\pi x=0$. Soit maintenant $n \geq 2$ (pour que la condition (5) soit vérifiée). On a $\epsilon(\pi)-\frac{\pi}{z} \neq 0$ et $\epsilon(\pi) \epsilon(x) \neq 0$ dans $\mathscr{D}_{n}(B, I)$ alors que $\pi x=0$. En effet $\delta\left(\tau\left(\epsilon(\pi)-\frac{\pi}{z}\right)\right)=\gamma(\pi)-\pi^{q-1}$ et $\delta(\tau(\epsilon(\pi) \epsilon(x)))=\gamma(\pi) \gamma(x)$. On notera que cet exemple rentre aussi dans le cadre des puissances divisées au sens de Grothendieck et Berthelot qui apparaîtront dans la définition 6.1.

De nouveau, soit $B$ une $\theta$-algèbre où l'image de $\pi$ est nilpotente, $I$ un idéal de $B$ et $n \in \mathbb{N}^{*}$. Soit $\left(M, \phi_{M}\right)$ un chtouca local minuscule sur $B / I$. On appellera relèvement de $\left(M, \phi_{M}\right)$ un chtouca local minuscule $\left(\widetilde{M}, \phi_{\widetilde{M}}\right)$ sur $B$ tel que $\widetilde{M}$ soit libre sur $\Theta \widehat{\otimes} B$, muni d'un isomorphisme de chtoucas locaux sur $B / I$ entre $\left(M, \phi_{M}\right)$ et le changement de base $\left(\widetilde{M}, \phi_{\widetilde{M}}\right)_{B / I}=\left(\widetilde{M} \otimes_{\vartheta \widehat{\otimes} B} \oslash \widehat{\otimes}(B / I), \phi_{\widetilde{M}} \otimes 1\right)$. On dira que $\left(M, \phi_{M}\right)$ est relevable s'il admet un relèvement $\left(\widetilde{M}, \phi_{\widetilde{M}}\right)$ au sens précédent. D'après le lemme $1.2,\left(M, \phi_{M}\right)$ est relevable localement pour la topologie de Zariski sur $\operatorname{Spec}(B / I)$.

On va construire un foncteur $\mathcal{F}$ de la catégorie des chtoucas locaux minuscules sur $B / I$ vers la catégorie des $\mathscr{D}_{n}(B, I)$-modules qui sont libres localement pour la topologie de Zariski sur Spec $B$, tel que si $\left(M, \phi_{M}\right)$ est un chtouca local minuscule sur $B / I$ relevable et si $\left(\widetilde{M}, \phi_{\widetilde{M}}\right)$ relève $\left(M, \phi_{M}\right)$,

$$
\mathcal{F}\left(M, \phi_{M}\right) \text { soit canoniquement isomorphe à } \widetilde{M} \otimes_{\vartheta \widehat{\otimes} B} \mathscr{D}_{n}(B, I) .
$$

La construction de $\mathcal{F}$ sera locale pour la topologie de Zariski sur $\operatorname{Spec}(B / I)$, de sorte qu'il suffira de travailler avec des chtoucas locaux minuscules sur $B / I$ relevables. Nous procéderons de la manière suivante. Soient

$$
f:\left(M_{1}, \phi_{M_{1}}\right) \rightarrow\left(M_{2}, \phi_{M_{2}}\right)
$$

un morphisme de chtoucas locaux minuscules sur $B / I$ relevables, $\left(\widetilde{M}_{i}, \phi_{\widetilde{M}_{i}}\right)$ un relèvement de $\left(M_{i}, \phi_{M_{i}}\right)$ pour $i=1,2$, et $\tilde{f}: \widetilde{M}_{1} \rightarrow \widetilde{M}_{2}$ un morphisme de $\theta \widehat{\otimes} B$-modules qui relève $f$ (l'existence de $\tilde{f}$ résulte de l'hypothèse que $\widetilde{M}_{1}$ est libre sur $\theta \widehat{\otimes} B$ ). Nous définirons par la formule (17) ci-dessous un morphisme de $\mathscr{D}_{n}(B, I)$-modules

$$
\mathcal{F}_{\left(\widetilde{M}_{2}, \phi_{\widetilde{M}_{2}}\right),\left(\widetilde{M}_{1}, \phi_{\widetilde{M}_{1}}\right)}(\widetilde{f}): \widetilde{M}_{1} \otimes_{\vartheta \widehat{\otimes} B} \mathscr{D}_{n}(B, I) \rightarrow \widetilde{M}_{2} \otimes_{\vartheta \widehat{\otimes} B} \mathscr{D}_{n}(B, I) .
$$

Nous démontrerons dans le lemme 5.8 que ce morphisme ne dépend pas du choix du relèvement $\tilde{f}$ si bien que nous le noterons $\mathcal{F}_{\left(\widetilde{M}_{2}, \phi_{\widetilde{M}_{2}}\right),\left(\widetilde{M}_{1}, \phi_{\widetilde{M}_{1}}\right)}(f)$. Nous démontrerons aussi dans le lemme 5.9 que lorsque $g:\left(M_{2}, \phi_{M_{2}}\right) \rightarrow\left(M_{3}, \phi_{M_{3}}\right)$ est un autre morphisme de chtoucas locaux minuscules sur $B / I$ relevables et lorsque $\left(\widetilde{M}_{3}, \phi_{\widetilde{M}_{3}}\right)$ est un relèvement de $\left(M_{3}, \phi_{M_{3}}\right)$ on a

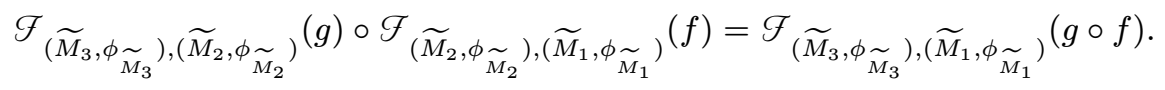

Dans le cas particulier où $\left(M_{1}, \phi_{M_{1}}\right)=\left(M_{2}, \phi_{M_{2}}\right)$ et où $f=\mathrm{Id}$, on obtiendra alors un isomorphisme $\mathcal{F}_{\left(\widetilde{M}_{2}, \phi_{\widetilde{M}_{2}}\right),\left(\widetilde{M}_{1}, \phi \widetilde{M}_{1}\right)}(\mathrm{Id})$ (car il résultera immédiatement de (17) que $\mathcal{F}_{\left(\widetilde{M}_{i}, \phi_{\widetilde{M}_{i}}\right),\left(\widetilde{M}_{i}, \phi_{M_{i}}\right)}(\mathrm{Id})=$ Id pour $\left.i=1,2\right)$. Par conséquent, à isomorphisme canonique 
près, $\widetilde{M} \otimes_{\oslash \widehat{\otimes} B} \mathscr{D}_{n}(B, I)$ ne dépendra que de $\left(M, \phi_{M}\right)$, ce qui autorisera à le noter $\mathcal{F}\left(M, \phi_{M}\right)$. Grâce à (7), $\mathcal{F}$ sera un foncteur de la catégorie des chtoucas locaux minuscules sur $B / I$ relevables vers celle des $\mathscr{D}_{n}(B, I)$-modules libres. Par recollement on obtiendra alors un foncteur $\mathcal{F}$ de la catégorie des chtoucas locaux minuscules sur $B / I$ vers celle des $\mathscr{D}_{n}(B, I)$-modules qui sont libres localement pour la topologie de Zariski sur Spec $B$.

Pour $i \in\{1,2\}$ on note $\psi_{\widetilde{M}_{i}}=(z-\pi)\left(\phi_{\widetilde{M}_{i}}\right)^{-1} \in \operatorname{Hom}_{\widehat{\partial} \widehat{\otimes}_{B}}\left(\widetilde{M}_{i},{ }^{\tau} \widetilde{M}_{i}\right)$. On voudrait poser

$$
\begin{aligned}
\mathcal{F}_{\left(\widetilde{M}_{2}, \phi_{\widetilde{M}_{2}}\right),\left(\widetilde{M}_{1}, \phi_{\widetilde{M}_{1}}\right)}(\widetilde{f}) \\
\quad=(z-\pi)^{-1} \cdots\left(z-\pi^{q^{n-1}}\right)^{-1} \phi_{\widetilde{M}_{2}} \cdots \tau^{n-1} \phi_{\widetilde{M}_{2}} \tau^{n}(\widetilde{f})^{\tau^{n-1}} \psi_{\widetilde{M}_{1}} \cdots \psi_{\widetilde{M}_{1}}
\end{aligned}
$$

mais le sens de cette expression n'est pas immédiat. Après une construction préliminaire nous

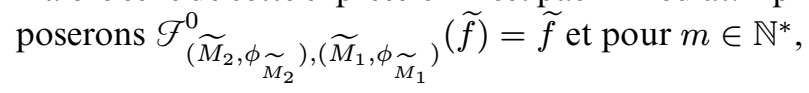

$$
\begin{aligned}
\mathcal{F}_{\left(\widetilde{M}_{2}, \phi_{\widetilde{M}_{2}}\right),\left(\widetilde{M}_{1}, \phi_{\widetilde{M}_{1}}\right)}(\widetilde{f}) & =(z-\pi)^{-1} \cdots\left(z-\pi^{q^{m-1}}\right)^{-1} \\
& \phi_{\widetilde{M}_{2}} \ldots \tau^{\tau^{m-2}} \phi_{\widetilde{M}_{2}} \tau^{m-1}\left(\phi_{\widetilde{M}_{2}}{ }^{\tau} \widetilde{f} \psi_{\widetilde{M}_{1}}-(z-\pi) \widetilde{f}\right)^{\tau^{m-2}} \psi_{\widetilde{M}_{1}} \cdots \psi_{\widetilde{M}_{1}}
\end{aligned}
$$

après avoir donné un sens au membre de droite à l'aide de $\epsilon$ et en utilisant le fait que $\phi_{\widetilde{M}_{2}} \tau \widetilde{f} \psi_{\widetilde{M}_{1}}-(z-\pi) \widetilde{f}$ est à coefficients dans $I$. Nous poserons alors

$$
\mathcal{F}_{\left(\widetilde{M}_{2}, \phi_{\widetilde{M}_{2}}\right),\left(\widetilde{M}_{1}, \phi_{\widetilde{M}_{1}}\right)}(\widetilde{f})=\sum_{m=0}^{n} \mathcal{F}_{\left(\widetilde{M}_{2}, \phi_{\widetilde{M}_{2}}\right),\left(\widetilde{M}_{1}, \phi_{\widetilde{M}_{1}}\right)}^{m}(\tilde{f}) .
$$

Nous vérifierons dans le lemme 5.8 que $\mathcal{F}_{\left(\widetilde{M}_{2}, \phi \widetilde{M}_{2}\right),\left(\widetilde{M}_{1}, \phi \widetilde{M}_{1}\right)}(\tilde{f})$ ne dépend pas du choix de $\tilde{f}$ et nous établirons ses propriétés dans le lemme 5.9. La construction du foncteur $\mathcal{F}$ sera alors achevée et celle de $\mathbb{D}$ sera une simple formalité.

Pour tout $m \in \mathbb{N}$, nous définissons $\epsilon_{m}: I \rightarrow \mathscr{D}_{n}(B, I)$ en posant

$$
\epsilon_{m}(x)=\left(1-\frac{\pi}{z}\right)^{-1}\left(1-\frac{\pi^{q}}{z}\right)^{-1} \cdots\left(1-\frac{\pi^{q^{m}}}{z}\right)^{-1} z^{q^{m}-m-1} \epsilon(x)^{q^{m}}
$$

pour $x \in I$. Pour $x \in I$ on a donc

$$
(z-\pi)\left(z-\pi^{q}\right) \cdots\left(z-\pi^{q^{m}}\right) \epsilon_{m}(x)=x^{q^{m}} .
$$

Remarque. - Pour $x \in I, \epsilon_{m}(x)$ joue donc le rôle de $\frac{x^{q^{m}}}{(z-\pi) \cdots\left(z-\pi^{q^{m}}\right)}$. Donc (avec la donnée supplémentaire de $(\hat{B}, \beta, \gamma)$ vérifiant la condition $(5)), \delta\left({ }^{\tau}\left(\epsilon_{m}(x)\right)\right) \in \hat{B}$ joue le rôle de $\frac{x^{q^{m+1}}}{\left(\pi-\pi^{q}\right) \cdots\left(\pi-\pi^{q^{m+1}}\right)}$ qui est un coefficient d'une série logarithme. Cela n'est pas surprenant, car des logarithmes apparaissent dans [33]. On aurait pu définir une $\Theta \widehat{\otimes} B$-algèbre par les générateurs $\epsilon_{m}(x)$ pour $m \in \mathbb{N}$ et $x \in I$, vérifiant certaines relations, mais comme $\epsilon_{m}(x)$ s'exprime simplement par (10) en termes de $\frac{\pi}{z}$ et $\epsilon(x)$, il nous a semblé plus économique de définir $\mathscr{D}_{n}(B, I)$ à l'aide des générateurs $\frac{\pi}{z}$ et $\epsilon(x)$ pour $x \in I$.

Soit $m \in \mathbb{N}$. On a $\epsilon_{m}(b x)=b^{q^{m}} \epsilon_{m}(x)$ pour $b \in B$ et $x \in I$, donc on étend $\epsilon_{m}$ par linéarité en un morphisme de $\theta \widehat{\otimes} B$-modules

$$
\check{\epsilon}_{m}: \tau^{m}(\Theta \widehat{\otimes} I) \rightarrow \mathscr{D}_{n}(B, I)
$$


en posant $\check{\epsilon}_{m}\left(\tau^{m}(a \otimes x)\right)=a \epsilon_{m}(x)$ pour $a \in \theta$ et $x \in I$. Pour $x \in \tau^{\tau^{m}}(\Theta \widehat{\otimes} I)$ on a

$$
(z-\pi)\left(z-\pi^{q}\right) \cdots\left(z-\pi^{q^{m}}\right) \check{\epsilon}_{m}(x)=\tau^{m}(x)
$$

où $\tau^{m}$ désigne ici le composé des trois morphismes $\theta \widehat{\otimes} B$-linéaires

$$
\tau^{m}(\Theta \widehat{\otimes} I) \rightarrow \tau^{\tau^{m}}(\Theta \widehat{\otimes} B) \stackrel{\tau^{m}}{\sim} \Theta \widehat{\otimes} B \rightarrow \mathscr{D}_{n}(B, I)
$$

Ces morphismes (et d'autres qui nous serviront ensuite) proviennent du diagramme commutatif de morphismes $\theta \widehat{\otimes} B$-linéaires

$$
\begin{aligned}
& { }^{\tau}(\theta \widehat{\otimes} I) \rightarrow{ }^{\tau}(\Theta \widehat{\otimes} B) \rightarrow{ }^{\tau} \mathscr{D}_{n}(B, I) \\
& \downarrow \tau \quad \downarrow \tau \tau \quad \downarrow \tau \\
& \theta \widehat{\otimes} I \subset \theta \widehat{\otimes} B \rightarrow \mathscr{D}_{n}(B, I)
\end{aligned}
$$

où $\tau:{ }^{\tau}(\theta \widehat{\otimes} B) \stackrel{\sim}{\rightarrow} \theta \widehat{\otimes} B$ envoie $^{\tau}(a \otimes b)$ sur $a \otimes b^{q}$, et $\tau:{ }^{\tau} \mathscr{D}_{n}(B, I) \rightarrow \mathscr{D}_{n}(B, I)$ envoie $\tau\left(\frac{\pi}{z}\right)$ sur $\frac{\pi^{q}}{z}$ et pour $x \in I$ envoie ${ }^{\tau}(\epsilon(x))$ sur $x^{q-1} \epsilon(x)=z^{q-1} \epsilon(x)^{q}$. On note que pour tout $x \in \Theta \widehat{\otimes} B$, on a $\tau\left({ }^{\tau} x\right)=(1 \widehat{\otimes} \operatorname{Fr})(x) \in \Theta \widehat{\otimes} B$.

Pour $m \geq n$ on a $\epsilon_{m}=0$ et $\check{\epsilon}_{m}=0$.

Soient $P$ et $Q$ des $\theta \widehat{\otimes} B$-modules libres localement pour la topologie de Zariski de Spec $B$

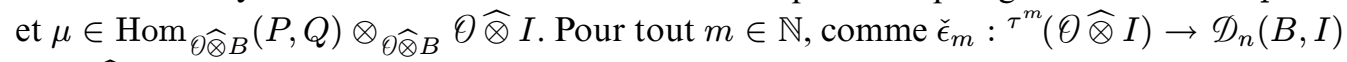
est $\theta \widehat{\otimes} B$-linéaire, on définit

$$
\check{\epsilon}_{m}\left({ }^{\tau^{m}} \mu\right) \in \operatorname{Hom}\left({ }^{\tau^{m}} P,{ }^{\tau^{m}} Q\right) \otimes_{\vartheta \widehat{\otimes} B} \mathscr{D}_{n}(B, I)
$$

sans ambiguïté comme l'image de $\tau^{\tau^{m}} \mu \in \operatorname{Hom}_{\partial \widehat{\otimes} B}\left(\tau^{m} P, \tau^{m} Q\right) \otimes_{\partial \widehat{\otimes} B} \tau^{m}(\vartheta \widehat{\otimes} I)$ par $\operatorname{Id}_{\operatorname{Hom}\left(\tau^{m} P, \tau^{m} Q\right)} \otimes_{\partial \widehat{\otimes} B} \check{\epsilon}_{m}$. Pour $m>0$ on a

$$
(z-\pi) \check{\epsilon}_{m}\left(\tau^{m} \mu\right)={ }^{\tau}\left(\check{\epsilon}_{m-1}\left(\tau^{m-1} \mu\right)\right)
$$

dans $\operatorname{Hom}\left(\tau^{m} P, \tau^{m} Q\right) \otimes_{\vartheta \widehat{\otimes} B} \mathscr{D}_{n}(B, I)$, où le membre de droite est implicitement l'extension des scalaires par le morphisme $\theta \widehat{\otimes} B$-linéaire $\tau:{ }^{\tau} \mathscr{D}_{n}(B, I) \rightarrow \mathscr{D}_{n}(B, I)$ qui figure dans le diagramme (11). Pour $m>0$ on a aussi

$$
\left(z-\pi^{q^{m}}\right) \check{\epsilon}_{m}\left(\tau^{m} \mu\right)=\check{\epsilon}_{m-1}\left(\tau^{\tau^{m}} \mu\right)
$$

dans $\operatorname{Hom}\left(\tau^{m} P,{ }^{\tau^{m}} Q\right) \otimes_{\emptyset \widehat{\otimes} B} \mathscr{D}_{n}(B, I)$, où l'expression ${ }^{\tau^{m}} \mu$ figurant dans le membre de droite est implicitement l'extension des scalaires par le morphisme $\theta \widehat{\otimes} B$-linéaire $\tau^{\tau^{m-1}}(\tau)$ de $\tau^{m}(\Theta \widehat{\otimes} I)={ }^{\tau^{m-1}}(\tau(\Theta \widehat{\otimes} I))$ vers ${ }^{\tau^{m-1}}(\Theta \widehat{\otimes} I)$.

Maintenant nous sommes outillés pour donner un sens aux formules heuristiques (8) et (9). Soit $\widetilde{f}: \widetilde{M}_{1} \rightarrow \widetilde{M}_{2}$ un morphisme de $\Theta \widehat{\otimes} B$-modules qui relève $f$. On pose

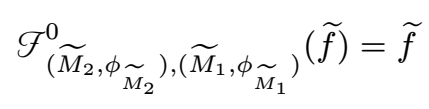


et pour $m \in \mathbb{N}^{*}$,

(14)

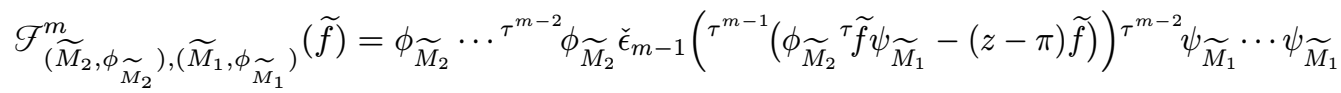

$$
\begin{aligned}
& =\phi_{\widetilde{M}_{2}} \cdots \tau^{m-2} \phi_{\widetilde{M}_{2}} \check{\epsilon}_{m-1}\left(\tau^{m-1}\left(\phi_{\widetilde{M}_{2}} \tau \widetilde{f}-\widetilde{f} \phi_{\widetilde{M}_{1}}\right)\right)^{\tau^{m-1}} \psi_{\widetilde{M}_{1}} \cdots \psi_{\widetilde{M}_{1}} \\
& =\phi_{\widetilde{M}_{2}} \cdots \tau^{\tau^{m-1}} \phi_{\widetilde{M}_{2}} \check{\epsilon}_{m-1}\left(\tau^{m-1}\left(\tau \widetilde{f} \psi_{\widetilde{M}_{1}}-\psi_{\widetilde{M}_{2}} \widetilde{f}\right)\right) \tau^{m-2} \psi_{\widetilde{M}_{1}} \cdots \psi_{\widetilde{M}_{1}} \\
& \in \operatorname{Hom}_{\widehat{\partial} \widehat{\otimes} B}\left(\widetilde{M}_{1}, \widetilde{M}_{2}\right) \otimes_{\vartheta \widehat{\otimes} B} \mathscr{D}_{n}(B, I) .
\end{aligned}
$$

Parmi ces trois formules nous privilégierons (15) car cela est préférable en vue d'un complément donné à la fin de ce paragraphe.

On note que $\left.\mathcal{F}_{\left(\widetilde{M}_{2}, \phi\right.}^{m}{\widetilde{M_{2}}}\right),\left(\widetilde{M}_{1}, \phi_{\widetilde{M}_{1}}\right)(\widetilde{f})=0$ pour $m>n$.

Pour tout $m \in \mathbb{N}$ on a

$$
\begin{gathered}
(z-\pi) \cdots\left(z-\pi^{q^{m-1}}\right)\left(\mathcal{F}_{\left(\widetilde{M}_{2}, \phi_{\widetilde{M}_{2}}\right),\left(\widetilde{M}_{1}, \phi_{\widetilde{M}_{1}}\right)}(\widetilde{f})+\cdots+\mathcal{F}_{\left(\widetilde{M}_{2}, \phi \widetilde{M}_{2}\right),\left(\widetilde{M}_{1}, \phi_{\widetilde{M}_{1}}\right)}^{m}(\widetilde{f})\right) \\
=\phi_{\widetilde{M}_{2}} \ldots \tau^{\tau^{m-1}} \phi_{\widetilde{M}_{2}} \tau^{m}(\widetilde{f})^{\tau^{m-1}} \psi_{\widetilde{M}_{1}} \cdots \psi_{\widetilde{M}_{1}},
\end{gathered}
$$

ce qui justifie l'intérêt de la notation suivante :

$$
\left.\mathcal{F}_{\left(\widetilde{M}_{2}, \phi_{\widetilde{M}_{2}}\right),\left(\widetilde{M}_{1}, \phi_{\widetilde{M}_{1}}\right)}(\widetilde{f})=\sum_{m=0}^{n} \mathcal{F}_{\left(\widetilde{M}_{2}, \phi\right.}^{m}{\widetilde{M_{2}}}\right),\left(\widetilde{M}_{1}, \phi_{\widetilde{M}_{1}}\right)(\widetilde{f}) .
$$

Lemme 5.8. - Le morphisme de $\mathscr{D}_{n}(B, I)$-modules

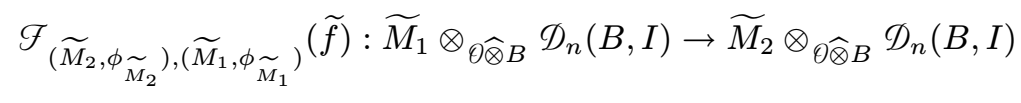

défini par (17) ne dépend pas du choix du relèvement $\tilde{f}$.

Démonstration. - Soit $\widetilde{f}^{\sharp}$ un autre relèvement de $f$ et

$$
K \in \operatorname{Hom}_{\emptyset \widehat{\otimes} B}\left(\widetilde{M}_{1}, \widetilde{M}_{2}\right) \otimes_{\emptyset \widehat{\otimes} B} \vartheta \widehat{\otimes} I
$$

tel que $\widetilde{f}^{\sharp}=\widetilde{f}+K$. Pour tout $m \in \mathbb{N}^{*}$ on a

$$
\begin{aligned}
\check{\epsilon}_{m-1}\left(\tau ^ { m - 1 } \left(\phi_{\widetilde{M}_{2}} \tau \widetilde{f}^{\sharp}\right.\right. & \left.\left.-\widetilde{f}^{\sharp} \phi_{\widetilde{M}_{1}}\right)\right)-\check{\epsilon}_{m-1}\left(\tau^{m-1}\left(\phi_{\widetilde{M}_{2}}{ }^{\tau} \widetilde{f}-\widetilde{f} \phi_{\widetilde{M}_{1}}\right)\right) \\
& =\check{\epsilon}_{m-1}\left(\tau^{m-1}\left(\phi_{\widetilde{M}_{2}}{ }^{\tau} K-K \phi_{\widetilde{M}_{1}}\right)\right) \\
& ={ }^{\tau^{m-1}} \phi_{\widetilde{M}_{2}} \check{\epsilon}_{m-1}\left(\tau^{m} K\right)-\check{\epsilon}_{m-1}\left(\tau^{m-1} K\right)^{\tau^{m-1}} \phi_{\widetilde{M}_{1}}
\end{aligned}
$$

Grâce à (13) on a $\check{\epsilon}_{m-1}\left(\tau^{m} K\right)=\left(z-\pi^{q^{m}}\right) \check{\epsilon}_{m}\left(\tau^{m} K\right)$, d'où, en utilisant (15),

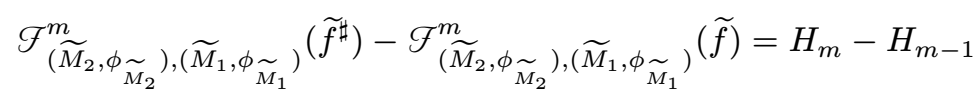

où l'on a posé

\section{Comme}

$$
H_{m}=\left(z-\pi^{q^{m}}\right) \phi_{\widetilde{M}_{2}} \cdots \tau^{\tau^{m-1}} \phi_{\widetilde{M}_{2}} \check{\epsilon}_{m}\left(\tau^{m} K\right)^{\tau^{m-1}} \psi_{\widetilde{M}_{1}} \cdots \psi_{\widetilde{M}_{1}} .
$$

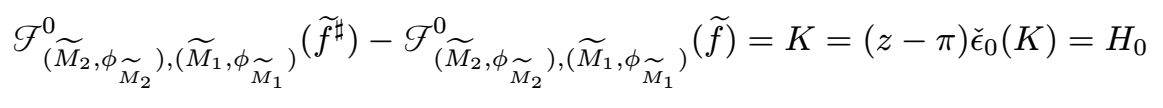


et comme $H_{n}=0$ on a bien montré

$$
\mathcal{F}_{\left(\widetilde{M}_{2}, \phi_{\widetilde{M}_{2}}\right),\left(\widetilde{M}_{1}, \phi_{\widetilde{M}_{1}}\right)}(\widetilde{f})=\mathcal{F}_{\left(\widetilde{M}_{2}, \phi_{\widetilde{M}_{2}}\right),\left(\widetilde{M}_{1}, \phi_{\widetilde{M}_{1}}\right)}\left(\widetilde{f}^{\sharp}\right) .
$$

Grâce au lemme précédent, on peut définir $\mathcal{F}_{\left(\widetilde{M}_{2}, \phi_{\widetilde{M}_{2}}\right),\left(\widetilde{M}_{1}, \phi_{\widetilde{M}_{1}}\right)}(f)$ comme $\mathcal{F}_{\left(\widetilde{M}_{2}, \phi_{\widetilde{M}_{2}}\right),\left(\widetilde{M}_{1}, \phi_{\widetilde{M}_{1}}\right)}(\widetilde{f})$ pour tout relèvement $\tilde{f}$ de $f$. Pour tout relèvement $\tilde{f}$ de $f$ on a donc

$$
\left.\mathcal{F}_{\left(\widetilde{M}_{2}, \phi_{\widetilde{M}_{2}}\right),\left(\widetilde{M}_{1}, \phi_{\widetilde{M}_{1}}\right)}(f)=\sum_{m=0}^{n} \mathcal{F}_{\left(\widetilde{M}_{2}, \phi\right.}^{m}{\widetilde{M_{2}}}\right),\left(\widetilde{M}_{1}, \phi_{\widetilde{M}_{1}}\right)(\widetilde{f}) .
$$

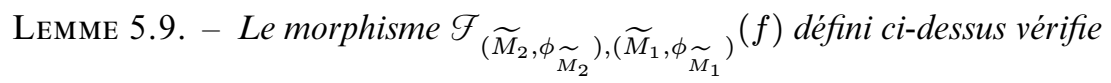

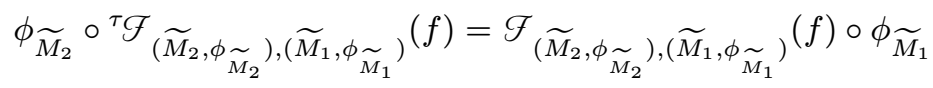

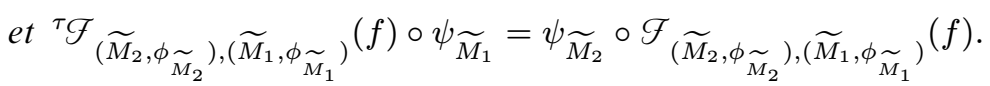

De plus la réduction modulo $\mathcal{J}_{n} d e \mathcal{F}_{\left(\widetilde{M}_{2},{\widehat{M_{2}}}_{2}\right),\left(\widetilde{M}_{1}, \phi_{\widetilde{M}_{1}}\right)}(f)$ est égale à $f \otimes_{\emptyset \widehat{\otimes}(B / I)} \operatorname{Id}_{\mathscr{D}_{n}(B, I) / \mathcal{J}_{n}}$. Enfin, si $\left(M_{3}, \phi_{M_{3}}\right)$ est un troisième chtouca local minuscule sur B/I relevable, $\left(\widetilde{M}_{3}, \phi_{\widetilde{M}_{3}}\right)$ un relèvement de $\left(M_{3}, \phi_{M_{3}}\right)$ et $g$ un morphisme de chtoucas locaux de $\left(M_{2}, \phi_{M_{2}}\right)$ vers $\left(M_{3}, \phi_{M_{3}}\right)$, on a

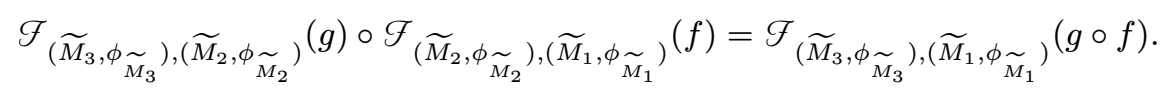

Dans les membres de gauche des égalités (19) et (20) on a utilisé implicitement l'extension des scalaires par le morphisme $\theta \widehat{\otimes} B$-linéaire $\tau:{ }^{\tau} \mathscr{D}_{n}(B, I) \rightarrow \mathscr{D}_{n}(B, I)$ introduit précédemment (comme dans (12)). La dernière assertion du lemme est la plus importante, car (21) montre que $\mathcal{F}$ est bien défini par (6) et est un foncteur de la catégorie des chtoucas locaux minuscules sur $B / I$ relevables vers la catégorie des $\mathscr{D}_{n}(B, I)$-modules libres. La preuve de (21) est calculatoire car on ne sait pas caractériser $\mathcal{F}_{\left(\widetilde{M}_{2}, \phi_{\widetilde{M}_{2}}\right),\left(\widetilde{M}_{1}, \phi_{\widetilde{M}_{1}}\right)}(f)$ par une propriété. En tous cas les deux premières assertions du lemme ne caractérisent pas $\mathcal{F}_{\left(\widetilde{M}_{2}, \phi_{\widetilde{M}_{2}}\right),\left(\widetilde{M}_{1}, \phi_{\widetilde{M}_{1}}\right)}(f)$ de manière unique. En effet il peut exister des éléments non nuls $y \in \mathscr{J}_{n}$ tels que $(z-\pi) y$ et $\tau\left({ }^{\tau} y\right)$ soient nuls dans $\mathscr{D}_{n}(B, I)$, comme on le voit en prenant $y=\epsilon(\pi) \epsilon(x)$ dans les notations de l'exemple 5.7.

Démonstration. - Soit $\tilde{f}$ un relèvement de $f$. Soit $m \in \mathbb{N}^{*}$. Grâce à (12) et (15) on obtient

$$
\begin{aligned}
\phi_{\widetilde{M}_{2}}{ }^{\tau} \mathcal{F}_{\left(\widetilde{M}_{2}, \phi_{\widetilde{M}_{2}}\right),\left(\widetilde{M}_{1}, \phi_{\widetilde{M}_{1}}\right)}(\widetilde{f}) \\
\quad=\phi_{\widetilde{M}_{2}} \ldots{ }^{\tau^{m-1}} \phi_{\widetilde{M}_{2}}{ }^{\tau}\left(\check{\epsilon}_{m-1}\left(\tau^{m-1}\left(\phi_{\widetilde{M}_{2}}{ }^{\tau} \widetilde{f}-\widetilde{f} \phi_{\widetilde{M}_{1}}\right)\right)\right) \tau^{m} \psi_{\widetilde{M}_{1}} \cdots{ }^{\tau} \psi_{\widetilde{M}_{1}} \\
=(z-\pi) \phi_{\widetilde{M}_{2}} \ldots{ }^{\tau^{m-1}} \phi_{\widetilde{M}_{2}} \check{\epsilon}_{m}\left(\tau^{m}\left(\phi_{\widetilde{M}_{2}}{ }^{\tau} \widetilde{f}-\widetilde{f} \phi_{\widetilde{M}_{1}}\right)\right) \tau^{m} \psi_{\widetilde{M}_{1}} \cdots{ }^{\tau} \psi_{\widetilde{M}_{1}} \\
=\mathcal{F}_{\left(\widetilde{M}_{2}, \phi_{\widetilde{M}_{2}}\right),\left(\widetilde{M}_{1}, \phi_{\widetilde{M}_{1}}\right)}(\widetilde{f}) \phi_{\widetilde{M}_{1}} .
\end{aligned}
$$


De plus

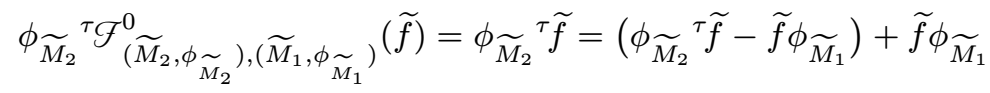

$$
\begin{aligned}
& =(z-\pi) \check{\epsilon}_{0}\left(\phi_{\widetilde{M}_{2}} \tau \widetilde{f}-\widetilde{f} \phi_{\widetilde{M}_{1}}\right)+\widetilde{f} \phi_{\widetilde{M}_{1}}=\check{\epsilon}_{0}\left(\phi_{\widetilde{M}_{2}} \tau \widetilde{f}-\widetilde{f} \phi_{\widetilde{M}_{1}}\right) \psi_{\widetilde{M}_{1}} \phi_{\widetilde{M}_{1}}+\widetilde{f} \phi_{\widetilde{M}_{1}} \\
& =\left(\mathcal{F}_{\left(\widetilde{M}_{2}, \phi_{\widetilde{M}_{2}}\right),\left(\widetilde{M}_{1}, \phi_{\widetilde{M}_{1}}\right)}(\widetilde{f})+\mathcal{F}_{\left(\widetilde{M}_{2}, \phi_{\widetilde{M}_{2}}\right),\left(\widetilde{M}_{1}, \phi_{\widetilde{M}_{1}}\right)}^{0}(\widetilde{f})\right) \phi_{\widetilde{M}_{1}} .
\end{aligned}
$$

Il résulte des deux suites d'égalités précédentes que pour tout $m \in \mathbb{N}$ on a

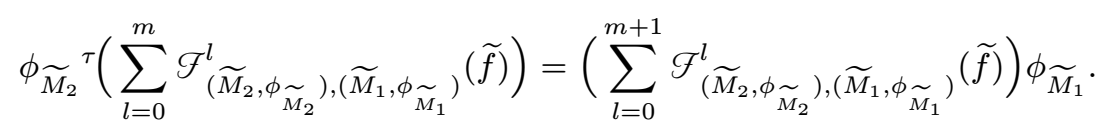

De la même façon (en utilisant (16) au lieu de (15)) on montre que pour tout $m \in \mathbb{N}$ on a

$$
\tau\left(\sum_{l=0}^{m} \mathcal{F}_{\left(\widetilde{M}_{2}, \phi_{\widetilde{M}_{2}}\right),\left(\widetilde{M}_{1}, \phi_{\widetilde{M}_{1}}\right)}^{l}(\widetilde{f})\right) \psi_{\widetilde{M}_{2}}=\psi_{\widetilde{M}_{1}}\left(\sum_{l=0}^{m+1} \mathcal{F}_{\left(\widetilde{M}_{2}, \phi_{\widetilde{M}_{2}}\right),\left(\widetilde{M}_{1}, \phi_{\widetilde{M}_{1}}\right)}^{l}(\widetilde{f})\right) .
$$

Les équations (19) et (20) résultent immédiatement de (22) et (23) en prenant $m=n$.

La deuxième assertion du lemme est claire car $\mathcal{F}_{\left(\widetilde{M}_{2}, \phi_{\widetilde{M}_{2}}\right),\left(\widetilde{M}_{1}, \phi_{\widetilde{M}_{1}}\right)}^{m}(\widetilde{f})$ appartient à $\operatorname{Hom}_{\vartheta \widehat{\otimes} B}\left(\widetilde{M}_{1}, \widetilde{M}_{2}\right) \otimes_{\vartheta \widehat{\otimes} B} \mathcal{J}_{n}$ pour tout $m \in \mathbb{N}^{*}$.

Il reste à montrer (21). Soient $\tilde{f}$ et $\widetilde{g}$ des relèvements de $f$ et $g$.

Pour $m \geq 0$ on a par définition, grâce à (15),

$$
\begin{aligned}
& \mathcal{F}_{\left(\widetilde{M}_{3}, \phi_{\widetilde{M}_{3}}\right),\left(\widetilde{M}_{2}, \phi_{\widetilde{M}_{2}}\right)}^{m}(\widetilde{g})\left(\sum_{l=0}^{m} \mathcal{F}_{\left(\widetilde{M}_{2}, \phi_{\widetilde{M}_{2}}\right),\left(\widetilde{M}_{1}, \phi_{\widetilde{M}_{1}}\right)}^{l}(\widetilde{f})\right) \\
& =\phi_{\widetilde{M}_{3}} \cdots \tau^{m-2} \phi_{\widetilde{M}_{3}} \check{\epsilon}_{m-1}\left(\tau^{m-1}\left(\phi_{\widetilde{M}_{3}}^{\tau} \widetilde{g}-\widetilde{g} \phi_{\widetilde{M}_{2}}\right)\right)^{\tau^{m-1}} \psi_{\widetilde{M}_{2}} \cdots \psi_{\widetilde{M}_{2}}\left(\sum_{l=0}^{m} \mathcal{F}_{\left(\widetilde{M}_{2}, \phi_{\widetilde{M}_{2}}\right),\left(\widetilde{M}_{1}, \phi_{\widetilde{M}_{1}}\right.}(\widetilde{f})\right)
\end{aligned}
$$

Or il résulte de (23) que

$$
\begin{aligned}
\tau^{m-1} \psi_{\widetilde{M}_{2}} \cdots \psi_{\widetilde{M}_{2}} & \left(\sum_{l=0}^{m} \mathcal{F}_{\left(\widetilde{M}_{2}, \phi\right.}^{l}{\widetilde{M_{2}}}\right),\left(\widetilde{M}_{1}, \phi_{\widetilde{M}_{1}}\right) \\
& \left.=\tau^{m} \mathcal{F}_{\left(\widetilde{M}_{2}, \phi\right.}^{0}(\widetilde{f})\right),\left(\widetilde{M}_{\widetilde{M}_{2}, \phi_{\widetilde{M}_{1}}}(\widetilde{f})^{\tau^{m-1}} \psi_{\widetilde{M}_{2}} \cdots \psi_{\widetilde{M}_{2}}=\tau^{m}(\widetilde{f})^{\tau^{m-1}} \psi_{\widetilde{M}_{2}} \cdots \psi_{\widetilde{M}_{2}} .\right.
\end{aligned}
$$

On en déduit

$$
\begin{aligned}
& \left.\sum_{l=0}^{m} \mathcal{F}_{\left(\widetilde{M}_{3}, \phi_{\widetilde{M}_{3}}\right),\left(\widetilde{M}_{2}, \phi_{\widetilde{M}_{2}}\right)}(\widetilde{g}) \mathcal{F}_{\left(\widetilde{M}_{2}, \phi\right.}^{l}{\widetilde{M_{2}}}\right),\left(\widetilde{M}_{1}, \phi_{\widetilde{M}_{1}}\right)(\widetilde{f}) \\
& =\phi_{\widetilde{M}_{3}} \ldots \tau^{m-2} \phi_{\widetilde{M}_{3}} \check{\epsilon}_{m-1}\left(\tau^{m-1}\left(\phi_{\widetilde{M}_{3}}^{\tau} \widetilde{g}-\widetilde{g} \phi_{\widetilde{M}_{2}}\right)\right)^{\tau^{m}}(\widetilde{f})^{\tau^{m-1}} \psi_{\widetilde{M}_{1}} \cdots \psi_{\widetilde{M}_{1}} \\
& =\phi_{\widetilde{M}_{3}} \cdots \tau^{\tau^{m-2}} \phi_{\widetilde{M}_{3}} \check{\epsilon}_{m-1}\left(\tau^{m-1}\left(\phi_{\widetilde{M}_{3}}^{\tau} \widetilde{g}^{\tau} \widetilde{f}-\widetilde{g} \phi_{\widetilde{M}_{2}} \tau \widetilde{f}\right)\right)^{\tau^{m-1}} \psi_{\widetilde{M}_{1}} \cdots \psi_{\widetilde{M}_{1}} .
\end{aligned}
$$


Pour $m>0$ on a par définition, grâce à (15),

$$
\begin{aligned}
& \left(\sum_{l=0}^{m-1} \mathcal{F}_{\left(\widetilde{M}_{3}, \phi_{\widetilde{M}_{3}}\right),\left(\widetilde{M}_{2}, \phi_{\widetilde{M}_{2}}\right)}^{l}(\widetilde{g})\right) \mathcal{F}_{\left(\widetilde{M}_{2}, \phi_{\widetilde{M}_{2}}\right),\left(\widetilde{M}_{1}, \phi_{\widetilde{M}_{1}}\right)}^{m}(\widetilde{f}) \\
= & \left(\sum_{l=0}^{m-1} \mathcal{F}_{\left(\widetilde{M}_{3}, \phi_{\widetilde{M}_{3}}\right),\left(\widetilde{M}_{2}, \phi_{\widetilde{M}_{2}}\right)}^{l}(\widetilde{g})\right) \phi_{\widetilde{M}_{2}} \ldots \tau^{m-2} \phi_{\widetilde{M}_{2}} \check{\epsilon}_{m-1}\left(\tau^{m-1}\left(\phi_{\widetilde{M}_{2}} \tau_{\tilde{f}}-\widetilde{f} \phi_{\widetilde{M}_{1}}\right)\right) \tau^{m-1} \psi_{\widetilde{M}_{1}} \cdots \psi_{\widetilde{M}_{1}} .
\end{aligned}
$$

Or il résulte de (22) que

$$
\begin{aligned}
& \left(\sum_{l=0}^{m-1} \mathcal{F}_{\left(\widetilde{M}_{3}, \phi_{\widetilde{M}_{3}}\right),\left(\widetilde{M}_{2}, \phi_{\widetilde{M}_{2}}\right)}(\widetilde{g})\right) \phi_{\widetilde{M}_{2}} \ldots \tau^{\tau^{m-2}} \phi_{\widetilde{M}_{2}} \\
& =\phi_{\widetilde{M}_{2}} \ldots \tau^{m-2} \phi_{\widetilde{M}_{2}} \tau^{m-1} \mathcal{F}_{\left(\widetilde{M}_{3}, \phi_{\widetilde{M}_{3}}\right),\left(\widetilde{M}_{2}, \phi_{\widetilde{M}_{2}}\right)}(\widetilde{g})=\phi_{\widetilde{M}_{2}} \ldots \tau^{m-2} \phi_{\widetilde{M}_{2}} \tau^{m-1}(\widetilde{g})
\end{aligned}
$$

d'où pour $m \in \mathbb{N}^{*}$,

$$
\begin{aligned}
\sum_{l=0}^{m-1} & \left.\mathcal{F}_{\left(\widetilde{M}_{3},{ }_{\widetilde{M}_{3}}\right),\left(\widetilde{M}_{2}, \phi_{\widetilde{M}_{2}}\right)}(\widetilde{g}) \mathcal{F}_{\left(\widetilde{M}_{2}, \phi_{\widetilde{M}_{2}}\right),\left(\widetilde{M}_{1}, \phi\right.}{\widetilde{M_{1}}}\right) \\
& =\phi_{\widetilde{M}_{3}} \ldots \tau^{m-2} \phi_{\widetilde{M}_{3}} \tau^{m-1}(\widetilde{g}) \check{\epsilon}_{m-1}\left(\tau^{m-1}\left(\phi_{\widetilde{M}_{2}} \tau \widetilde{f}-\widetilde{f} \phi_{\widetilde{M}_{1}}\right)\right) \tau^{m-1} \psi_{\widetilde{M}_{1}} \cdots \psi_{\widetilde{M}_{1}} \\
& =\phi_{\widetilde{M}_{3}} \ldots \tau^{m-2} \phi_{\widetilde{M}_{3}} \check{\epsilon}_{m-1}\left(\tau^{m-1}\left(\widetilde{g} \phi_{\widetilde{M}_{2}}{ }^{\tau} \widetilde{f}-\widetilde{g} \widetilde{f} \phi_{\widetilde{M}_{1}}\right)\right) \tau^{m-1} \psi_{\widetilde{M}_{1}} \cdots \psi_{\widetilde{M}_{1}} .
\end{aligned}
$$

Comme

$$
\left(\phi_{\widetilde{M}_{3}} \tau^{\tau} \widetilde{f}-\widetilde{g} \phi_{\widetilde{M}_{2}} \tau \widetilde{f}\right)+\left(\widetilde{g} \phi_{\widetilde{M}_{2}}{ }^{\tau} \widetilde{f}-\widetilde{g} \widetilde{f} \phi_{\widetilde{M}_{1}}\right)=\phi_{\widetilde{M}_{3}}{ }^{\tau}(\widetilde{g} \widetilde{f})-\widetilde{g} \widetilde{f} \phi_{\mathbb{M}_{1}}
$$

on déduit de (24) et (25) et de la formule (15) appliquée à $\tilde{g} \tilde{f}$ que pour tout $m \in \mathbb{N}$,

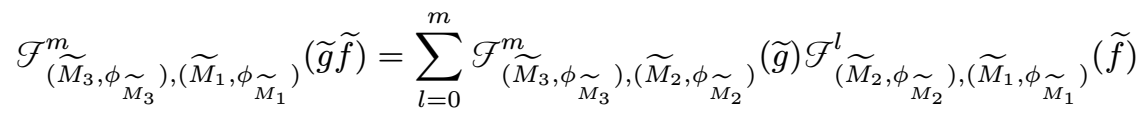

$$
\begin{aligned}
& \left.+\sum_{l=0}^{m-1} \mathcal{F}_{\left(\widetilde{M}_{3}, \phi\right.}^{l}{\widetilde{M_{3}}}\right),\left(\widetilde{M}_{2}, \phi_{\widetilde{M}_{2}}\right)(\widetilde{g}) \mathcal{F}_{\left(\widetilde{M}_{2}, \phi_{\widetilde{M}_{2}}\right),\left(\widetilde{M}_{1}, \phi_{\widetilde{M}_{1}}\right)}^{m}(\widetilde{f})
\end{aligned}
$$

et (21) en résulte aussitôt.

Remarque. - La légère dissymétrie de la démonstration de (21) vient du choix de la décomposition

$$
\{0, \ldots, m\}^{2} \backslash\{0, \ldots, m-1\}^{2}=\{m\} \times\{0, \ldots, m\} \cup\{0, \ldots, m-1\} \times\{m\} .
$$

On aurait pu choisir la décomposition $\{m\} \times\{0, \ldots, m-1\} \cup\{0, \ldots, m\} \times\{m\}$ et utiliser (16) plutôt que (15) dans les calculs.

On a donc construit, pour toute $\theta$-algèbre $B$ où l'image de $\pi$ est nilpotente, tout idéal $I$ de $B$ et tout $n \in \mathbb{N}^{*}$, un foncteur $\mathcal{F}$ de la catégorie des chtoucas locaux minuscules sur $B / I$ vers la catégorie des $\mathscr{D}_{n}(B, I)$-modules libres localement pour la topologie de Zariski sur Spec $B$. Voici maintenant la construction de $\mathbb{D}$ : pour tout chtouca local minuscule $\left(M, \phi_{M}\right)$ sur $A$ 
on définit un $\vartheta$-cristal $\mathbb{D}\left(M, \phi_{M}\right)$ sur $A$ en posant, pour tout $A$-objet test $(B, I, s, \hat{B}, \beta, \gamma)$ et pour tout entier $n$ tel que la condition (5) soit vérifiée,

$$
\left(\mathbb{D}\left(M, \phi_{M}\right)\right)(B, I, s, \hat{B}, \beta, \gamma)={ }^{\tau}\left(\mathcal{F}\left(M, \phi_{M}\right)_{B / I}\right) \otimes_{\tau \mathscr{D}_{n}(B, I)} \hat{B}
$$

où $\mathcal{F}$ dépend de $n, B, I$ et où le produit tensoriel utilise le morphisme de $\theta \widehat{\otimes} B$-algèbres $\delta:{ }^{\tau} \mathscr{D}_{n}(B, I) \rightarrow \hat{B}$ construit précédemment à partir de la donnée de $n, B, I,(\hat{B}, \beta, \gamma)$. On vérifie facilement que $\left(\mathbb{D}\left(M, \phi_{M}\right)\right)(B, I, s, \hat{B}, \beta, \gamma)$ ne dépend pas du choix de l'entier $n$ et que $\mathbb{D}\left(M, \phi_{M}\right)$ est un $\theta$-cristal. On vérifie enfin que $\mathbb{D}$ est un foncteur.

Démonstration de la proposition 5.6. - On vérifie immédiatement que $\mathbb{D}$ est compatible aux images inverses. Enfin si $\pi=0$ dans $A,\left({ }^{\tau} M,{ }^{\tau} \phi_{M}\right)$ est un chtouca local minuscule sur $A$ et $\phi_{M}$ est une isogénie de $\left({ }^{\tau} M,{ }^{\tau} \phi_{M}\right)$ vers $\left(M, \phi_{M}\right)$ donc par fonctorialité de $\mathbb{D}, \mathbb{D}\left(M, \phi_{M}\right)$ est un $F$-cristal. Cela termine la preuve de la proposition 5.6.

Remarque. - Pour récapituler, lorsque $\left(M, \phi_{M}\right)$ est un chtouca local minuscule sur $A$, $(B, I, s, \hat{B}, \beta, \gamma)$ est un $A$-objet test et $\left(\widetilde{M}, \phi_{\widetilde{M}}\right)$ est un relèvement de $\left(M, \phi_{M}\right)_{B / I}$, on a posé

$$
\left(\mathbb{D}\left(M, \phi_{M}\right)\right)(B, I, s, \hat{B}, \beta, \gamma)=\left({ }^{\tau} \widetilde{M} /(z-\pi)^{\tau} \widetilde{M}\right) \otimes_{B} \hat{B}={ }^{\tau} \widetilde{M} \otimes_{\vartheta \widehat{\otimes} B} \hat{B}
$$

(où le morphisme $\theta \widehat{\otimes} B \rightarrow \hat{B}$ est donné par $a \widehat{\otimes} b \mapsto a \beta(b)$ ). Si $\left(\widetilde{M}_{1}, \phi_{\widetilde{M}_{1}}\right)$ et $\left(\widetilde{M}_{2}, \phi_{\widetilde{M}_{2}}\right)$ sont deux relèvements de $\left(M, \phi_{M}\right)_{B / I}$ et $n \in \mathbb{N}^{*}$ est tel que la condition (5) soit vérifiée, on a identifié $\widetilde{T}_{1} \otimes_{\partial \widehat{\otimes} B} \hat{B}$ à $\widetilde{M}_{2} \otimes_{\partial \widehat{\otimes} B} \hat{B}$ par l'image de

$$
\tau\left(\mathcal{F}_{\left(\widetilde{M}_{2}, \phi_{\widetilde{M}_{2}}\right),\left(\widetilde{M}_{1}, \phi_{\widetilde{M}_{1}}\right)}(\mathrm{Id})\right) \in \operatorname{Hom}_{\partial \widehat{\otimes} B}\left({ }^{\tau} \widetilde{M}_{1},{ }^{\tau} \widetilde{M}_{2}\right) \otimes_{\partial \widehat{\otimes} B}{ }^{\tau}\left(\mathscr{D}_{n}(B, I)\right)
$$

dans $\operatorname{Hom}_{\vartheta \widehat{\otimes} B}\left({ }^{\tau} \widetilde{M}_{1},{ }^{\tau} \widetilde{M}_{2}\right) \otimes_{\partial \widehat{\otimes} B} \hat{B} \operatorname{par} \delta$.

La proposition suivante énonce que $\mathbb{D}$ est compatible à la dualité de Cartier. Soit $\left(M, \phi_{M}\right)$ un chtouca local minuscule sur $A$. On note $\psi_{M}=(z-\pi) \phi_{M}^{-1}$ et $M^{*}=\operatorname{Hom}_{\partial \widehat{\otimes} A}(M, \oslash \widehat{\otimes} A)$. Alors $\left(M^{*},{ }^{t} \psi_{M}\right)$ est aussi un chtouca local minuscule sur $A$, que l'on doit considérer comme le dual de Cartier de $\left(M, \phi_{M}\right)$ (si on normalise la dualité de Cartier par le choix de $(\Theta \widehat{\otimes} A,(z-\pi))$ comme chtouca local «de Lubin-Tate »). On remarque que $\psi_{M^{*}}={ }^{t} \phi_{M}$, autrement dit la dualité de Cartier permute $\phi$ et $\psi$.

Proposition 5.10. - Le Ө-cristal $\mathbb{D}\left(M^{*},{ }^{t} \psi_{M}\right)$ s'identifie au dual du $\theta$-cristal $\mathbb{D}\left(M, \phi_{M}\right)$ de façon fonctorielle par rapport à $\left(M, \phi_{M}\right)$. Autrement dit pour tout A-objet test $(B, I, s, \hat{B}, \beta, \gamma)$,

$$
\left(\mathbb{D}\left(M^{*},{ }^{t} \psi_{M}\right)\right)(B, I, s, \hat{B}, \beta, \gamma)=\operatorname{Hom}_{\hat{B}}\left(\left(\mathbb{D}\left(M, \phi_{M}\right)\right)(B, I, s, \hat{B}, \beta, \gamma), \hat{B}\right) .
$$

Démonstration. - Cela résulte immédiatement du fait que la construction de $\mathbb{D}$ est symétrique en $\phi$ et $\psi$.

La fin de ce paragraphe peut être sautée en première lecture. 


\subsection{Un point de vue complémentaire sur la construction du foncteur de Dieudonné}

Soient $B$ une $\theta$-algèbre où l'image de $\pi$ est nilpotente et $I$ un idéal de $B$. Soit $n \in \mathbb{N}^{*}$. On se replace dans le cadre de la construction du foncteur $\mathcal{F}$. La formule (16) montre que pour $x \in \widetilde{M}_{1}, \mathcal{F}_{\left(\widetilde{M}_{2}, \phi_{\widetilde{M}_{2}}\right),\left(\widetilde{M}_{1}, \phi_{\widetilde{M}_{1}}\right)}(\widetilde{f})(x) \in \widetilde{M}_{2} \otimes \mathscr{D}_{n}(B, I)$ est de la forme $a_{0}+\phi_{\widetilde{M}_{2}} \check{\epsilon}_{0}\left(a_{1}\right)+$ $\phi_{\widetilde{M}_{2}}{ }^{\tau} \phi_{\widetilde{M}_{2}} \check{\epsilon}_{1}\left(a_{2}\right)+\cdots$ avec $a_{0} \in \widetilde{M}_{2}, a_{1} \in{ }^{\tau} \widetilde{M}_{2} \otimes(\theta \widehat{\otimes} I), a_{2} \in \tau^{2} \widetilde{M}_{2} \otimes \tau(\theta \widehat{\otimes} I) \cdots$ (tous les produits tensoriels sont $\operatorname{sur} \theta \widehat{\otimes} B$ ). Explicitement on a

$$
a_{0}=\tilde{f} x \text { et } a_{m}=\tau^{m-1}\left(\tau \tilde{f} \psi_{\widetilde{M}_{1}}-\psi_{\widetilde{M}_{2}} \widetilde{f}\right)^{\tau^{m-2}} \psi_{\widetilde{M}_{1}} \cdots \psi_{\widetilde{M}_{1}} x \text { pour } m>0 .
$$

Soit $\left(\widetilde{M}, \phi_{\widetilde{M}}\right)$ un chtouca local sur $B$ relevant $\left(M, \phi_{M}\right)$. Heuristiquement on va définir un $\oslash \widehat{\otimes} B$-module $\mathscr{G}\left(\widetilde{M}, \phi_{\widetilde{M}}\right)$ comme l'ensemble des sommes infinies

$$
a_{0}+\phi_{\widetilde{M}} \frac{a_{1}}{z-\pi}+\phi_{\widetilde{M}}^{\tau} \phi_{\widetilde{M}} \frac{a_{2}}{(z-\pi)\left(z-\pi^{q}\right)}+\cdots
$$

avec $a_{0} \in \widetilde{M}, a_{1} \in \widetilde{T} \otimes(\Theta \widehat{\otimes} I), a_{2} \in \tau^{2} \widetilde{M} \otimes{ }^{\tau}(\Theta \widehat{\otimes} I), \ldots$ On formalise ceci en définissant $\mathscr{G}\left(\widetilde{M}, \phi_{\widetilde{M}}\right)$ comme le conoyau de l'application

$$
T_{\psi_{\widetilde{M}}}=\prod_{m \in \mathbb{N}} \tau^{m} \widetilde{M} \otimes \tau^{m}(\theta \widehat{\otimes} I) \rightarrow \widetilde{M} \oplus \prod_{m \in \mathbb{N}^{*}} \tau^{m} \widetilde{M} \otimes \tau^{m-1}(\theta \widehat{\otimes} I)
$$

donnée par la matrice indexée par $\mathbb{N} \times \mathbb{N}$

$$
\left(\begin{array}{ccccc}
1 & 0 & 0 & 0 & \cdots \\
-\psi_{\widetilde{M}} & 1 & 0 & 0 & \ddots \\
0 & -{ }^{\tau} \psi_{\widetilde{M}} & 1 & 0 & \ddots \\
0 & 0 & -\tau^{2} \psi_{\widetilde{M}} & 1 & \ddots \\
\vdots & \ddots & \ddots & \ddots & \ddots
\end{array}\right)
$$

où les 1 sur la diagonale sont en fait l'inclusion $\widetilde{M} \otimes(\theta \widehat{\otimes} I) \rightarrow \widetilde{M}$ et

$$
1 \otimes \tau^{\tau^{m-1}} \tau: \tau^{m} \widetilde{M} \otimes \tau^{m}(\Theta \widehat{\otimes} I) \rightarrow{ }^{\tau^{m}} \widetilde{M} \otimes \tau^{\tau^{m-1}}(\Theta \widehat{\otimes} I) \text { pour } m>0
$$

et les termes sous-diagonaux sont en fait

$$
-\tau^{\tau^{m}} \psi_{\widetilde{M}} \otimes 1: \tau^{\tau^{m}} \widetilde{M} \otimes \tau^{\tau^{m}}(\Theta \widehat{\otimes} I) \rightarrow{ }^{\tau^{m+1}} \widetilde{M} \otimes \tau^{\tau^{m}}(\Theta \widehat{\otimes} I) \text { pour } m \geq 0 .
$$

On a un morphisme $u_{\left(\widetilde{M}, \phi_{\widetilde{M}}\right)}: \mathscr{G}\left(\widetilde{M}, \phi_{\widetilde{M}}\right) \rightarrow \widetilde{M} \otimes \mathscr{D}_{n}(B, I)$ qui à $\left(a_{0}, a_{1}, a_{2}, \ldots\right)$ associe

$$
a_{0}+\phi_{\widetilde{M}_{2}} \check{\epsilon}_{0}\left(a_{1}\right)+\phi_{\widetilde{M}_{2}}^{\tau} \phi_{\widetilde{M}_{2}} \check{\epsilon}_{1}\left(a_{2}\right)+\cdots
$$

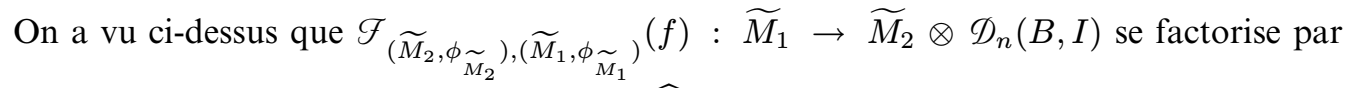
$u_{\left(\widetilde{M}_{2}, \phi \widetilde{M}_{2}\right.}$. En fait on a un morphisme de $\Theta \widehat{\otimes} B$-modules

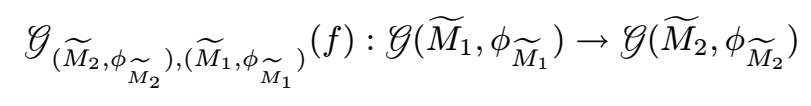


compatible à la composition, et tel que le diagramme suivant soit commutatif

$$
\begin{aligned}
& \mathscr{G}\left(\widetilde{M}_{1}, \phi_{\widetilde{M}_{1}}\right) \longrightarrow \stackrel{\mathscr{G}\left(\widetilde{M}_{2}, \phi \widetilde{M}_{2}\right),\left(\widetilde{M}_{1}, \phi \widetilde{M}_{1}\right)^{(f)}}{\longrightarrow} \mathscr{G}\left(\widetilde{M}_{2}, \phi_{\widetilde{M}_{2}}\right)
\end{aligned}
$$

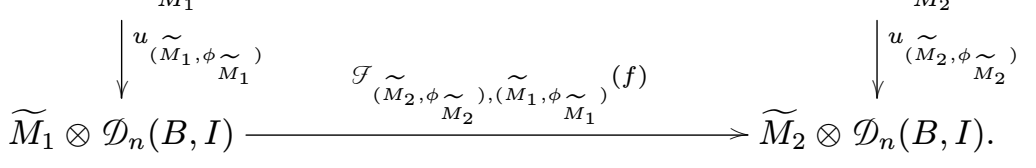

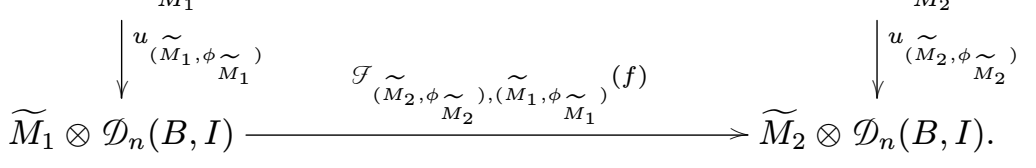

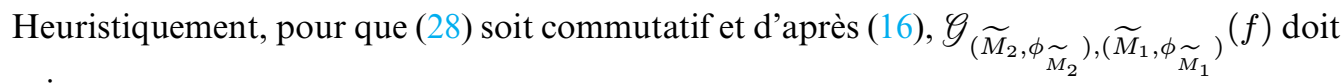
agir par

$$
F=\tilde{f}+\phi_{\widetilde{M}_{2}} \frac{\tau \widetilde{f} \psi_{\widetilde{M}_{1}}-\psi_{\widetilde{M}_{2}} \tilde{f}}{z-\pi}+\phi_{\widetilde{M}_{2}}{ }^{\tau} \phi_{\widetilde{M}_{2}} \frac{\tau\left(\tau \widetilde{f} \psi_{\widetilde{M}_{1}}-\psi_{\widetilde{M}_{2}} \widetilde{f}\right) \psi_{\widetilde{M}_{1}}}{(z-\pi)\left(z-\pi^{q}\right)}+\cdots
$$

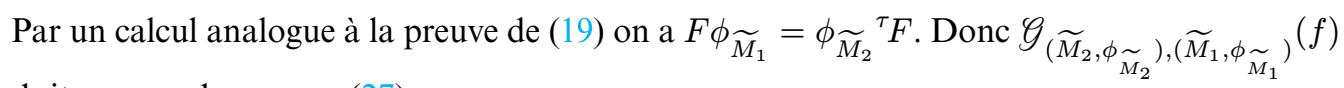
doit envoyer la somme (27) sur

$$
F a_{0}+\phi_{\widetilde{M}_{2}} \frac{\tau F a_{1}}{z-\pi}+\phi_{\widetilde{M}_{2}}{ }^{\tau} \phi_{\widetilde{M}_{2}} \frac{\tau^{2} F a_{2}}{(z-\pi)\left(z-\pi^{q}\right)}+\cdots
$$

que l'on développe en utilisant (29). On définit donc $\mathscr{G}_{\left(\widetilde{M}_{2}, \phi_{\widetilde{M}_{2}}\right),\left(\widetilde{M}_{1}, \phi_{\widetilde{M}_{1}}\right)}(f)$ par passage au quotient à l'aide du diagramme commutatif

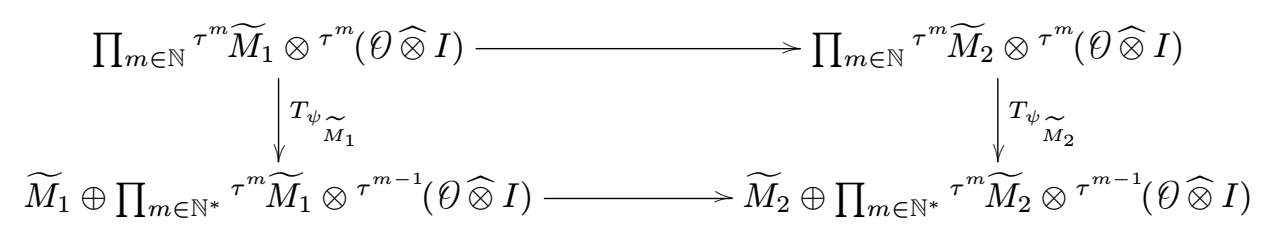

où la ligne du dessus est donnée par la matrice diagonale indexée par $\mathbb{N} \times \mathbb{N}$ dont les coefficients sont $\widetilde{f},{ }^{\tau} \widetilde{f},{ }^{2} \widetilde{f}, \ldots$ et où la ligne du dessous est donnée par la matrice indexée $\operatorname{par} \mathbb{N} \times \mathbb{N}$

$$
\left(\begin{array}{cccc}
\tilde{f} & 0 & 0 & \cdots \\
\tau \widetilde{f} \psi_{\widetilde{M}_{1}}-\psi_{\widetilde{M}_{2}} \widetilde{f} & \tau \widetilde{f} & 0 & \ddots \\
\tau\left(\tau \widetilde{f} \psi_{\widetilde{M}_{1}}-\psi_{\widetilde{M}_{2}} \widetilde{f}\right) \psi_{\widetilde{M}_{1}} & \tau\left(\tau \widetilde{f} \psi_{\widetilde{M}_{1}}-\psi_{\widetilde{M}_{2}} \widetilde{f}\right) & \tau^{2} \widetilde{f} & \ddots \\
\tau^{2}\left(\tau \widetilde{f} \psi_{\widetilde{M}_{1}}-\psi_{\widetilde{M}_{2}} \widetilde{f}\right)^{\tau} \psi_{\widetilde{M}_{1}} \psi_{\widetilde{M}_{1}} \tau^{2}\left(\tau \widetilde{f} \psi_{\widetilde{M}_{1}}-\psi_{\widetilde{M}_{2}} \widetilde{f}\right)^{\tau} \psi_{\widetilde{M}_{1}} \tau^{2}\left(\tau \widetilde{f} \psi_{\widetilde{M}_{1}}-\psi_{\widetilde{M}_{2}} \widetilde{f}\right) & \ddots \\
\vdots & \ddots & \ddots & \ddots
\end{array}\right)
$$

On montre facilement que le morphisme ainsi défini ne dépend pas du choix de $\widetilde{f}$. Un calcul mécanique montre qu'il est compatible à la composition. Il en résulte que $\mathscr{G}\left(\widetilde{M}, \phi_{\widetilde{M}}\right)$ dépend seulement du chtouca local $\left(M, \phi_{M}\right)$ sur $B / I$, et de façon fonctorielle. La commutativité du diagramme (28) est justifiée par un calcul dont l'heuristique a été donnée ci-dessus (en utilisant (15) et (19)). 


\subsection{Comparaison avec la construction de Messing}

Nous allons comparer notre construction avec celle de Messing [48]. D'après [2], la théorie de l'extension vectorielle universelle se transpose en égales caractéristiques. En fait tous les arguments de [48] se transcrivent facilement en termes de modules de coordonnées. Nous donnerons des démonstrations indépendantes plus naturelles dans notre contexte. Soient $B$ une $\theta$-algèbre où l'image de $\pi$ est nilpotente, $\left(M, \phi_{M}\right)$ un chtouca local minuscule sur $B$ et $G$ le $\theta$-module divisible qui lui est associé. On rappelle que $G=\lim G_{n}$ avec $G_{n}=\operatorname{Gr}\left(M / z^{n} M\right)$ et $M / z^{n} M=\operatorname{Hom}_{\mathbb{F}_{q}}\left(G_{n}, \mathbb{G}_{a}\right)$ (cf. la proposition 0.3). On rappelle d'après [26] que lorsque $H$ est un $\theta$-module (limite inductive de sous- $\theta$-modules de $\pi$-torsion), son module de coordonnées $\mathcal{H}=\operatorname{Hom}_{\mathbb{F}_{q}}\left(H, \mathbb{G}_{a}\right)$ est muni d'une action de $\mathscr{\Theta} \widehat{\otimes} B$ provenant de l'action de $\theta$ sur $H$ et de celle de $B$ sur $\mathbb{G}_{a}$ par homothéties, et que le Frobenius ${ }^{\tau} \mathcal{H} \rightarrow \mathcal{H}$ provient du morphisme de Frobenius $x \mapsto x^{q}$ de $\mathbb{G}_{a}$. Si en fait l'action de $\theta$ est stricte (voir [21]), d'après le paragraphe 3.2 de [1] celle-ci induit un morphisme $\psi_{\mathscr{H}}: \mathscr{H} \rightarrow \tau \mathscr{H}$ vérifiant $\phi_{\mathscr{H}} \psi_{\mathscr{H}}=(z-\pi) \operatorname{Id}_{\mathscr{H}}$ et $\psi_{\mathscr{H}} \phi_{\mathscr{H}}=(z-\pi) \operatorname{Id}_{\tau \mathscr{H}}$.

Suivant Grothendieck, pour tout $B$-module localement libre $N$ on note $\mathbb{V}(N)=\operatorname{Spec}\left(\bigoplus_{n \in \mathbb{N}} \operatorname{Sym}^{n} N\right)$ l'espace total de $N^{*}$. On le munit de l'action de $\theta$ par homothéties et on appelle $\theta$-module vectoriel un $\theta$-module de cette forme. Le module de coordonnées $V(N)$ de $\mathbb{V}(N)$ est $\operatorname{Hom}_{\mathbb{F}_{q}}\left(\mathbb{V}(N), \mathbb{G}_{a}\right)=\bigoplus_{n \in \mathbb{N}} \tau^{n} N$, où la structure de $\theta \widehat{\otimes} B$-module sur $N$ vient du morphisme $\theta \widehat{\otimes} B \rightarrow B$ qui envoie $z$ sur $\pi$. On note que $N \subset \bigoplus_{n \in \mathbb{N}} \tau^{n} N$ est formé des morphismes linéaires de $\mathbb{V}(N)$ dans $\mathbb{G}_{a}$. De plus $\phi_{\mathcal{V}}\left(a_{0}, a_{1}, \ldots\right)=\left(0, a_{0}, a_{1}, \ldots\right)$ et $\psi_{\mathcal{V}}\left(a_{0}, a_{1}, \ldots\right)=\left((z-\pi) a_{1},(z-\pi) a_{2}, \ldots\right)$ de sorte que $\operatorname{Ker} \psi_{v}$ contient la partie linéaire $N$.

On appelle extension vectorielle de $G$ par un $\vartheta$-module vectoriel $V=\mathbb{V}(N)$ un $\vartheta$-module de la forme $E=\lim E_{n}$ où $E_{n}$ est une extension de $G_{n}$ par $V$ dans la catégorie des schémas en

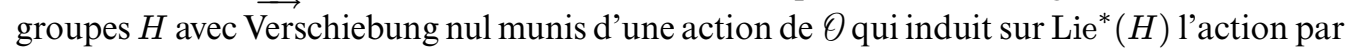
homothéties (ou plutôt, pour être précis, munis d'une action stricte de $\Theta$ [21]). On définit le module de coordonnées $\mathcal{E}$ de $E$ comme $\mathscr{E}=\lim _{\longleftarrow} \operatorname{Hom}_{\mathbb{F}_{q}}\left(E_{n}, \mathbb{G}_{a}\right)$. On a alors une suite exacte

$$
0 \rightarrow M \stackrel{j}{\rightarrow} \mathcal{E} \rightarrow V(N) \rightarrow 0
$$

On note $\mathcal{E}_{\text {lin }}$ l'image inverse de la partie linéaire $N \subset \mathcal{V}(N)$ dans cette suite exacte de sorte que $\mathcal{E}_{\text {lin }}$ est un sous- $\Theta \widehat{\otimes} B$-module de $\mathcal{E}$. On remarque que $\psi_{\mathcal{E}}\left(\mathcal{E}_{\text {lin }}\right) \subset{ }^{\tau} M \stackrel{\tau_{j}}{\hookrightarrow}{ }^{\tau} \mathcal{E}$ et on note $\psi_{\mathcal{E}_{\text {lin }}}: \mathcal{E}_{\text {lin }} \rightarrow{ }^{\tau} M$ la restriction de $\psi_{\mathcal{E}}$ à $\mathscr{E}_{\text {lin }}$.

Le foncteur contravariant qui à $E$ associe $\mathcal{E}$ est une équivalence de la catégorie des extensions vectorielles de $G$ vers la catégorie $\mathscr{E} x t$ des $\left(N, \mathcal{E}, \phi_{\mathscr{E}}, \psi_{\mathscr{E}}\right)$ où $N$ est un $B$-module localement libre et $\left(\mathcal{E}, \phi_{\mathcal{E}}, \psi_{\varepsilon}\right)$ est une extension de $\left(V(N), \phi_{\mathcal{V}}, \psi_{V}\right) \operatorname{par}\left(M, \phi_{M}, \psi_{M}\right)$ dans la catégorie des $\left(\mathcal{H}, \phi_{\mathscr{H}}, \psi_{\mathscr{H}}\right)$ où $\mathcal{H}$ est un $\theta \widehat{\otimes} B$-module et $\phi_{\mathscr{H}}:{ }^{\tau} \mathcal{H} \rightarrow \mathcal{H}$ et $\psi_{\mathscr{H}}: \mathcal{H} \rightarrow \tau \mathscr{H}$ sont tels que $\phi_{\mathscr{H}} \psi_{\mathcal{H}}=z-\pi$ et $\psi_{\mathscr{H}} \phi_{\mathscr{H}}=z-\pi$. Pour montrer que ce foncteur est une équivalence de catégories on utilise le paragraphe 3.2 de [1]. Le foncteur inverse est obtenu en généralisant un peu le foncteur Gr de l'introduction. Pour tout $n \in \mathbb{N}$ on note $\left(\mathscr{E} / z^{n} M\right)^{*}=\operatorname{Hom}_{B}\left(\mathscr{E} / z^{n} M, B\right)$ et alors

$$
E_{n}=\operatorname{Ker}\left({ }^{t} \phi_{\mathscr{E}}-\sigma_{\left(\mathscr{E} / z^{n} M\right)^{*} / \operatorname{Spec} B}:\left(\mathcal{E} / z^{n} M\right)^{*} \rightarrow \sigma^{*}\left(\left(\mathcal{E} / z^{n} M\right)^{*}\right)\right) .
$$

Soit $\mathscr{E} x t_{\text {lin }}$ la catégorie des $\left(N, \mathscr{E}_{\text {lin }}, \psi_{\text {lin }}\right)$ où $N$ est un $B$-module localement libre (considéré comme $\theta \widehat{\otimes} B$-module grâce au morphisme $\theta \widehat{\otimes} B \rightarrow B$ qui envoie $z$ sur $\pi$ ), Élin est une 
extension $0 \rightarrow M \stackrel{j_{\text {lin }}}{\rightarrow} \mathcal{E}_{\text {lin }} \rightarrow N \rightarrow 0$ dans la catégorie des $\theta \widehat{\otimes} B$-modules et $\psi_{\text {lin }}: \mathcal{E}_{\text {lin }} \rightarrow{ }^{\tau} M$ est tel que $\psi_{\text {lin }} j_{\text {lin }}=\psi_{M}: M \rightarrow{ }^{\tau} M$ et que $(z-\pi)=j_{\text {lin }} \phi_{M} \psi_{\text {lin }}: \mathcal{E}_{\text {lin }} \rightarrow \mathcal{E}_{\text {lin }}$. Le foncteur de $\mathcal{E} x t$ dans $\mathscr{E} x t_{\text {lin }}$ qui à $\left(N, \mathcal{E}, \phi_{\delta}, \psi_{\mathscr{E}}\right)$ associe $\left(N, \mathcal{E}_{\text {lin }}, \psi_{\text {lin }}\right)$ est une équivalence de catégories. En effet dans les notations précédentes, le morphisme

$$
\begin{aligned}
\mathcal{E}_{\operatorname{lin}} \oplus^{\tau} \mathcal{E}_{\operatorname{lin}} \oplus^{\tau^{2}} \mathcal{E}_{\operatorname{lin}} \oplus \cdots & \rightarrow \mathcal{E} \\
\left(a_{1}, a_{2}, a_{3}, \ldots\right) & \mapsto a_{1}+\phi_{\mathcal{E}}\left(a_{2}\right)+{ }^{\tau} \phi_{\mathcal{E}} \phi_{\mathcal{E}}\left(a_{3}\right)
\end{aligned}
$$

est surjectif (puisque $\phi_{\mathcal{V}}$ agit sur $\mathcal{V}(N)=\bigoplus_{n \in \mathbb{N}} \tau^{n} N$ par le décalage à droite) et la relation $\phi_{E}{ }^{\tau} j_{\text {lin }}=j_{\text {lin }} \phi_{M}$ fournit des générateurs évidents pour son noyau. On a donc un isomorphisme canonique entre $\mathcal{E}$ et le conoyau de l'application

$$
\begin{aligned}
\bigoplus_{n \in \mathbb{N}^{*}} \tau^{n} M & \rightarrow \bigoplus_{n \in \mathbb{N}^{*}} \tau^{n-1} \mathcal{E}_{\text {lin }} \\
\left(b_{1}, b_{2}, b_{3}, \ldots\right) & \mapsto\left(\left(j_{\operatorname{lin}} \phi_{M}\right) b_{1},-{ }^{\tau} j_{\operatorname{lin}} b_{1}+{ }^{\tau}\left(j_{\operatorname{lin}} \phi_{M}\right) b_{2},-{ }^{\tau^{2}} j_{\operatorname{lin}} b_{2}+{ }^{2}\left(j_{\operatorname{lin}} \phi_{M}\right) b_{3}, \ldots\right) .
\end{aligned}
$$

Le morphisme $\phi_{\mathscr{E}}:{ }^{\tau} \mathcal{E} \rightarrow \mathcal{E}$ est induit par le morphisme de complexes donné par les applications

$$
\begin{aligned}
& \bigoplus_{n \in \mathbb{N}^{*}} \tau^{n+1} M \rightarrow \bigoplus_{n \in \mathbb{N}^{*}} \tau^{n} M,\left(a_{1}, a_{2}, a_{3}, \ldots\right) \mapsto\left(0, a_{1}, a_{2}, \ldots\right) \\
& \text { et } \bigoplus_{n \in \mathbb{N}^{*}} \tau^{n} \mathcal{E}_{\operatorname{lin}} \rightarrow \bigoplus_{n \in \mathbb{N}^{*}} \tau^{n-1} \mathcal{E}_{\operatorname{lin}},\left(a_{1}, a_{2}, a_{3}, \ldots\right) \mapsto\left(0, a_{1}, a_{2}, \ldots\right)
\end{aligned}
$$

et $\psi_{\mathscr{E}}: \mathscr{E} \rightarrow{ }^{\tau} \mathcal{E}$ est induit par le morphisme de complexes donné par les applications

$$
\begin{aligned}
\bigoplus_{n \in \mathbb{N}^{*}} \tau^{n} M & \rightarrow \bigoplus_{n \in \mathbb{N}^{*}}{ }^{\tau^{n+1}} M \\
\left(a_{1}, a_{2}, a_{3}, \ldots\right) & \mapsto\left((z-\pi) a_{2},(z-\pi) a_{3}, \ldots\right) \\
\text { et } \bigoplus_{n \in \mathbb{N}^{*}} \tau^{n-1} \mathcal{E}_{\operatorname{lin}} & \rightarrow \bigoplus_{n \in \mathbb{N}^{*}} \tau^{n} \mathcal{E}_{\operatorname{lin}} \\
\left(a_{1}, a_{2}, a_{3}, \ldots\right) & \mapsto\left({ }^{\tau} j_{\operatorname{lin}} \psi_{\operatorname{lin}}\left(a_{1}\right)+(z-\pi) a_{2},(z-\pi) a_{3}, \ldots\right) .
\end{aligned}
$$

Ces formules fournissent un foncteur quasi-inverse (la relation $(z-\pi)=j_{\text {lin }} \phi_{M} \psi_{\text {lin }}$ garantit que $\left.\phi_{\delta} \psi_{\varepsilon}=(z-\pi)\right)$.

D'autre part pour $\left(N, \mathcal{E}_{\text {lin }}, \psi_{\text {lin }}\right)$ dans $\varepsilon x t_{\text {lin }}, \psi_{\text {lin }}: \mathcal{E}_{\text {lin }} \rightarrow{ }^{\tau} M$ passe au quotient et fournit un morphisme $u$ de $N=\varepsilon_{\operatorname{lin}} / j_{\text {lin }}(M)$ vers ${ }^{\tau} M / \psi_{M}(M)$. On a donc un foncteur de $\varepsilon x t_{\text {lin }}$ dans la catégorie des couples $(N, u)$ où $N$ est un $B$-module localement libre et $u$ est un morphisme $B$-linéaire de $N$ dans ${ }^{\tau} M / \psi_{M}(M)$. Ce foncteur est une équivalence de catégories et un foncteur quasi-inverse envoie $(N, u) \operatorname{sur}\left(N, \mathcal{E}_{\text {lin }}, \psi_{\text {lin }}\right)$ avec

$$
\begin{gathered}
\mathcal{E}_{\operatorname{lin}}=\left\{(x, y) \in N \oplus{ }^{\tau} M, u(x)=y \bmod \psi_{M}(M)\right\}, \\
\psi_{\operatorname{lin}}(x, y)=y \text { et } j_{\operatorname{lin}}(x)=\left(0, \psi_{M}(x)\right) .
\end{gathered}
$$

Enfin la catégorie des couples $(N, u)$ comme ci-dessus admet $\left({ }^{\tau} M / \psi_{M}(M)\right.$, Id) comme objet final. La catégorie $\delta x t_{\text {lin }}$ admet donc comme objet final $\left({ }^{\tau} M / \psi_{M}(M), \mathcal{E}_{\text {lin }}\left(M, \phi_{M}\right), \psi_{\text {lin }}\right)$ avec $\mathcal{E}_{\operatorname{lin}}\left(M, \phi_{M}\right)={ }^{\tau} M, j_{\text {lin }}=\psi_{M}$ et $\psi_{\text {lin }}=\operatorname{Id}_{\tau_{M}}$. Par conséquent la catégorie $\varepsilon x t$ 
admet comme objet final $\left({ }^{\tau} M / \psi_{M}(M), \mathcal{E}\left(M, \phi_{M}\right), \phi_{\mathscr{E}}, \psi_{\varepsilon}\right)$ où $\mathcal{E}\left(M, \phi_{M}\right)$ est le conoyau de l'application

$$
\begin{aligned}
\bigoplus_{n \in \mathbb{N}^{*}} \tau^{n} M & \rightarrow \bigoplus_{n \in \mathbb{N}^{*}} \tau^{n} M \\
\left(b_{1}, b_{2}, b_{3}, \ldots\right) & \mapsto\left((z-\pi) b_{1},-{ }^{\tau} \psi_{M} b_{1}+\left(z-\pi^{q}\right) b_{2},-{ }^{\tau^{2}} \psi_{M} b_{2}+\left(z-\pi^{q^{2}}\right) b_{3}, \ldots\right) .
\end{aligned}
$$

D'après (31) et (32), $\phi_{\mathscr{E}}:{ }^{\tau} \mathcal{E}\left(M, \phi_{M}\right) \rightarrow \mathcal{E}\left(M, \phi_{M}\right)$ est induit par le morphisme de complexes donné par les applications

$$
\begin{aligned}
& \left(a_{1}, a_{2}, a_{3}, \ldots\right) \mapsto\left(0, a_{1}, a_{2}, \ldots\right) \text { sur la source et } \\
& \left(a_{1}, a_{2}, a_{3}, \ldots\right) \mapsto\left(0, a_{1}, a_{2}, \ldots\right) \text { sur le but. }
\end{aligned}
$$

D'après (33) et (34), $\psi_{\mathscr{E}}: \mathcal{E}\left(M, \phi_{M}\right) \rightarrow{ }^{\tau} \mathcal{E}\left(M, \phi_{M}\right)$ est induit par le morphisme de complexes donné par les applications

$$
\begin{aligned}
& \left(a_{1}, a_{2}, a_{3}, \ldots\right) \mapsto\left((z-\pi) a_{2},(z-\pi) a_{3}, \ldots\right) \text { sur la source et } \\
& \left(a_{1}, a_{2}, a_{3}, \ldots\right) \mapsto\left({ }^{\tau} \psi_{M}\left(a_{1}\right)+(z-\pi) a_{2},(z-\pi) a_{3}, \ldots\right) \text { sur le but. }
\end{aligned}
$$

On a bien $\phi_{\delta} \psi_{\mathscr{E}}=(z-\pi)$ et $\psi_{\delta} \phi_{\mathscr{E}}=(z-\pi)$.

Il existe donc une extension vectorielle universelle $0 \rightarrow V(G) \rightarrow E(G) \rightarrow G \rightarrow 0$, telle que toute extension vectorielle $0 \rightarrow V \rightarrow E \rightarrow G \rightarrow 0$ soit son image directe par un unique morphisme $V(G) \rightarrow V$. Le module de coordonnées de $E(G)$ est $\mathcal{E}\left(M, \phi_{M}\right)$. Le dual de l'algèbre de Lie de $E(G)$ est $\mathcal{E}\left(M, \phi_{M}\right) / \phi_{\mathcal{E}}\left({ }^{\tau} \mathcal{E}\left(M, \phi_{M}\right)\right)$ qui s'identifie canoniquement à ${ }^{\tau} M /(z-\pi)^{\tau} M \operatorname{par}\left(a_{1}, a_{2}, \ldots\right) \mapsto a_{1} \bmod (z-\pi)^{\tau} M$. La suite exacte $0 \rightarrow V(G) \rightarrow E(G) \rightarrow$ $G \rightarrow 0$ correspond à une suite exacte

$$
0 \rightarrow M \stackrel{j_{M}}{\rightarrow} \mathcal{E}\left(M, \phi_{M}\right) \stackrel{p_{M}}{\rightarrow} \mathcal{V}\left(M, \phi_{M}\right) \rightarrow 0
$$

où $j_{M}(m)=\left(\psi_{M}(m), 0,0, \ldots\right)$. On rappelle que $V\left(M, \phi_{M}\right)=\bigoplus_{n \in \mathbb{N}} \tau^{n}\left({ }^{\tau} M / \psi_{M}(M)\right)$ avec $\phi_{V}: \tau \mathcal{V}\left(M, \phi_{M}\right) \rightarrow \mathcal{V}\left(M, \phi_{M}\right)$ agissant par décalage à droite. Heuristiquement on peut considérer $\mathcal{E}\left(M, \phi_{M}\right)$ comme l'ensemble des sommes finies

$$
\phi_{M} \frac{a_{1}}{z-\pi}+\phi_{M}{ }^{\tau} \phi_{M} \frac{a_{2}}{z-\pi^{q}}+\phi_{M}{ }^{\tau} \phi_{M}{ }^{2} \phi_{M} \frac{a_{3}}{z-\pi^{q^{2}}}+\cdots \text { avec } a_{i} \in{ }^{\tau^{i}} M .
$$

Supposons maintenant que $I$ est un idéal de $B$ muni de puissances divisées nilpotentes $\gamma$ au sens de Grothendieck et Berthelot, comme dans la définition 6.1. Pour tout $n \in \mathbb{N}$, on définit $\gamma_{n}: I \rightarrow I$ en posant

$$
\gamma_{n}(x)=\left(\pi^{q^{n}-q^{n-1}}-1\right)^{-1} \cdots\left(\pi^{q^{n}-1}-1\right)^{-1} \gamma \circ \cdots \circ \gamma(x) \text { (où } \gamma \text { est appliqué } n \text { fois). }
$$

Donc $\gamma_{0}(x)=x$ et $\gamma_{n}(x)$ joue le rôle de $\frac{x^{q^{n}}}{\left(\pi^{q^{n}}-\pi^{q^{n-1}}\right) \cdots\left(\pi^{q^{n}}-\pi\right)}$. Pour tout $n \in \mathbb{N}$, on définit aussi $\lambda_{n}: I \rightarrow I$ en posant

$$
\lambda_{n}(x)=\left(1-\pi^{q-1}\right)^{-1} \cdots\left(1-\pi^{q^{n}-1}\right)^{-1} \pi^{\left(q^{n-1}+\cdots+1\right)-n} \gamma \circ \cdots \circ \gamma(x)
$$

(où $\gamma$ est appliqué $n$ fois) de sorte que $\lambda_{0}(x)=x$ et $\lambda_{n}(x)$ joue le rôle de $\frac{x^{q^{n}}}{\left(\pi-\pi^{q}\right) \cdots\left(\pi-\pi^{q^{n}}\right)}$.

La série $\exp (x)=\sum_{n \in \mathbb{N}} \gamma_{n}(x)$ est analogue à l'exponentielle $\sum \frac{x^{n}}{n !}$, de la même façon que la série $\log (x)=\sum_{n \in \mathbb{N}} \lambda_{n}(x)$ est analogue au logarithme. Plus précisément soit $L T$ le Ө-module formel de Lubin-Tate, qui est égal à $\hat{\mathbb{G}}_{a}$ comme groupe formel avec action de $\mathbb{F}_{q}$ 
et sur lequel $\pi \in \Theta$ agit par $x \mapsto \pi x+x^{q}$. En d'autres termes $L T$ est le $\theta$-module formel associé au chtouca local $(\theta \widehat{\otimes} B, z-\pi)$. Alors

$$
\begin{aligned}
& \log : \operatorname{Ker}(L T(B) \rightarrow L T(B / I)) \rightarrow \operatorname{Ker}\left(\hat{\mathbb{G}}_{a}(B) \rightarrow \hat{\mathbb{G}}_{a}(B / I)\right) \\
& \text { et } \quad \exp : \operatorname{Ker}\left(\hat{\mathbb{G}}_{a}(B) \rightarrow \hat{\mathbb{G}}_{a}(B / I)\right) \rightarrow \operatorname{Ker}(L T(B) \rightarrow L T(B / I))
\end{aligned}
$$

sont $\theta$-linéaires.

Transposons en termes de modules de coordonnées l'analogue en égales caractéristiques de [48]. Par $\theta$-linéarité on étend $\gamma_{n}$ et $\lambda_{n}$ en

$$
\breve{\gamma}_{n}: \tau^{n}(\theta \widehat{\otimes} I) \rightarrow \theta \widehat{\otimes} I \text { et } \breve{\lambda}_{n}: \tau^{n}(\theta \widehat{\otimes} I) \rightarrow \theta \widehat{\otimes} I \text {. }
$$

Si $\mathcal{H}$ est un $\theta \widehat{\otimes} B$-module, $\phi_{\mathcal{H}}:{ }^{\tau} \mathcal{H} \rightarrow \mathscr{H}$ et $\psi_{\mathcal{H}}: \mathcal{H} \rightarrow{ }^{\tau} \mathcal{H}$ sont des morphismes $\theta \widehat{\otimes} B$-linéaires vérifiant $\phi_{\mathscr{H}} \psi_{\mathscr{H}}=z-\pi$ et $\psi_{\mathscr{H}} \phi_{\mathscr{H}}=z-\pi$, et si $N$ est un $B$-module localement libre, on appelle exponentielle un morphisme $\eta: \mathcal{H} \rightarrow \mathcal{V}(N)=\bigoplus_{n \in \mathbb{N}} \tau^{n} N$ de la forme

$$
\eta(x)=\left(\theta(x), \breve{\gamma}_{1}\left({ }^{\tau} \theta\left(\psi_{\mathcal{H}}(x)\right), \breve{\gamma}_{2}\left({ }^{\tau^{2}} \theta\left({ }^{\tau} \psi_{\mathcal{H}} \psi_{\mathscr{H}}(x)\right), \ldots\right)\right.\right.
$$

où $\theta: \mathcal{H} / \phi_{\mathscr{H}}\left({ }^{\tau} \mathcal{H}\right) \rightarrow N \otimes_{B} I$ est arbitraire (on remarque que $\eta \phi_{\mathscr{H}}=\phi_{V}{ }^{\tau} \eta$ et ${ }^{\tau} \eta \psi_{\mathcal{H}}=\psi_{V} \eta$ et que $\eta$ est $\theta \widehat{\otimes} B$-linéaire).

Soient $f:\left(M_{1}, \phi_{M_{1}}\right) \rightarrow\left(M_{2}, \phi_{M_{2}}\right)$ un morphisme de chtoucas locaux minuscules sur $B / I$, et $\left(\widetilde{M}_{1}, \phi_{\widetilde{M}_{1}}\right)$ et $\left(\widetilde{M}_{2}, \phi_{\widetilde{M}_{2}}\right)$ des relèvements à $B$. Il existe un unique morphisme de $\oslash \widehat{\otimes} B$-modules

$$
\mathcal{E}_{\left(\widetilde{M}_{2}, \phi_{\widetilde{M}_{2}}\right),\left(\widetilde{M}_{1}, \phi_{\widetilde{M}_{1}}\right)}(f): \mathcal{E}\left(\widetilde{M}_{1}, \phi_{\widetilde{M}_{1}}\right) \rightarrow \mathcal{E}\left(\widetilde{M}_{2}, \phi_{\widetilde{M}_{2}}\right)
$$

vérifiant $\left.\phi_{\mathcal{E}}^{\tau} \mathcal{E}_{\left(\widetilde{M}_{2}, \phi\right.} \widetilde{M}_{2}\right),\left(\widetilde{M}_{1}, \phi_{\widetilde{M}_{1}}\right)(f)=\mathcal{E}_{\left(\widetilde{M}_{2},{ }_{\widetilde{M}_{2}}\right),\left(\widetilde{M}_{1}, \phi_{\widetilde{M}_{1}}\right)}(f) \phi_{\mathscr{E}}$, relevant le morphisme induit par $f$ modulo $I$, et déterminé de manière unique par la condition suivante : pour tout morphisme de $B$-modules $h:{ }^{\tau} \widetilde{M}_{1} / \psi_{\widetilde{M}_{1}}\left(\widetilde{M}_{1}\right) \rightarrow{ }^{\tau} \widetilde{M}_{2} / \psi_{\widetilde{M}_{2}}\left(\widetilde{M}_{2}\right)$ dont la réduction modulo $I$ est ${ }^{\tau} f$, le morphisme

$$
p_{\widetilde{M}_{2}} \circ \mathcal{E}_{\left(\widetilde{M}_{2}, \phi_{\widetilde{M}_{2}}\right),\left(\widetilde{M}_{1}, \phi_{\widetilde{M}_{1}}\right)}(f)-\left(h,{ }^{\tau} h, \ldots\right) \circ p_{\widetilde{M}_{1}}: \mathcal{E}\left(\widetilde{M}_{1}, \phi_{\widetilde{M}_{1}}\right) \rightarrow \bigoplus_{n \in \mathbb{N}} \tau^{n}\left(\widetilde{M}_{2} / \psi\left(\widetilde{M}_{2}\right)\right)
$$

est une exponentielle. Nous donnerons plus loin une formule pour $\mathcal{E}_{\left(\widetilde{M}_{2}, \phi_{\widetilde{M}_{2}}\right),\left(\widetilde{M}_{1}, \phi \widetilde{M}_{1}\right)}(f)$.

Pour tout relèvement $\left(\widetilde{M}, \phi_{\widetilde{M}}\right)$ on a une application $\kappa_{\left(\widetilde{M}, \phi_{\widetilde{M}}\right)}: \mathscr{G}\left(\widetilde{M}, \phi_{\widetilde{M}}\right) \rightarrow \mathcal{E}\left(\widetilde{M}, \phi_{\widetilde{M}}\right)$ telle que le diagramme suivant soit commutatif

$$
\begin{aligned}
& \mathscr{G}\left(\widetilde{M}_{1}, \phi_{\widetilde{M}_{1}}\right) \stackrel{\mathscr{G}\left(\widetilde{M}_{2}, \phi_{\widetilde{M}_{2}}\right),\left(\widetilde{M}_{1}, \phi \widetilde{M}_{1}\right)}{(f)} \mathscr{G}\left(\widetilde{M}_{2}, \phi_{\widetilde{M}_{2}}\right)
\end{aligned}
$$

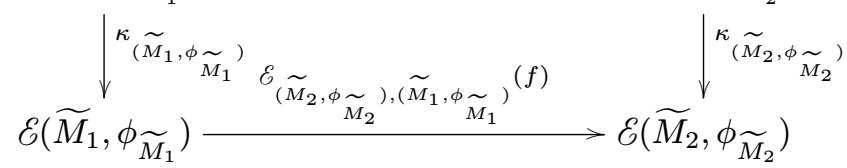

Heuristiquement $\kappa_{\left(\widetilde{M}, \phi_{\widetilde{M}}\right)}$ doit envoyer une somme infinie

$$
a_{0}+\phi_{\widetilde{M}} \frac{a_{1}}{z-\pi}+\phi_{\widetilde{M}}{ }^{\tau} \phi_{\widetilde{M}} \frac{a_{2}}{(z-\pi)\left(z-\pi^{q}\right)}+\phi_{\widetilde{M}^{\tau}} \phi_{\widetilde{M}^{2}}^{\tau^{2}} \phi_{\widetilde{M}} \frac{a_{3}}{(z-\pi)\left(z-\pi^{q}\right)\left(z-\pi^{q^{2}}\right)}+\cdots
$$


avec $a_{0} \in \widetilde{M}, a_{1} \in{ }^{\tau} \widetilde{M} \otimes(\theta \widehat{\otimes} I), a_{2} \in \tau^{2} \widetilde{M} \otimes{ }^{\tau}(\theta \widehat{\otimes} I), \ldots$ sur une somme finie

$$
\phi_{\widetilde{M}} \frac{b_{1}}{z-\pi}+\phi_{\widetilde{M}}^{\tau} \phi_{\widetilde{M}} \frac{b_{2}}{z-\pi^{q}}+\phi_{\widetilde{M}}{ }^{\tau} \phi_{\widetilde{M}} \tau^{2} \phi_{\widetilde{M}} \frac{b_{3}}{z-\pi^{q^{2}}}+\cdots \text { avec } b_{i} \in \tau^{i} \widetilde{M}
$$

Comme $a_{n+1}=\left(\pi^{q^{n}}-\pi^{q^{n-1}}\right) \cdots\left(\pi^{q^{n}}-\pi\right) \breve{\gamma}_{n}\left(a_{n+1}\right)$ les puissances divisées au sens de Grothendieck et Berthelot fournissent exactement les puissances de $\pi$ nécessaires pour effectuer la décomposition en éléments simples. Soient $\mu_{k, n} \in \theta$ pour $0 \leq k \leq n$ tels que

$$
\frac{\left(\pi^{q^{n}}-\pi^{q^{n-1}}\right) \cdots\left(\pi^{q^{n}}-\pi\right)}{(z-\pi)\left(z-\pi^{q}\right) \cdots\left(z-\pi^{q^{n}}\right)}=\sum_{k=0}^{n} \frac{\mu_{k, n}}{z-\pi^{q^{k}}} .
$$

On note désormais $\mu_{k, n}$ l'image de $\mu_{k, n}$ dans $B$. On a $\mu_{n, n}=1$ et grâce à l'hypothèse que $\pi$ est nilpotent dans $B, \mu_{k, n}=0$ si $n-k$ est assez grand. Pour $x \in \tau^{n}(\Theta \widehat{\otimes} I)$ on a $\mu_{0, n} \breve{\gamma}_{n}(x)=\breve{\lambda}_{n}(x)$ et plus généralement $\mu_{k, n} \breve{\gamma}_{n}(x)=\breve{\gamma}_{k}\left(\breve{\lambda}_{n-k}(x)\right)$.

On définit alors $\kappa_{\left(\widetilde{M}, \phi_{\widetilde{M}}\right)} \operatorname{par} \kappa_{\left(\widetilde{M}, \phi_{\widetilde{M}}\right)}\left(a_{0}, a_{1}, \ldots\right)=\left(b_{1}, b_{2}, \ldots\right)$ avec

$$
\begin{aligned}
b_{1} & =\psi_{\widetilde{M}}\left(a_{0}\right)+a_{1}+{ }^{\tau} \phi_{\widetilde{M}}\left(\breve{\lambda}_{1}\left(a_{2}\right)\right)+{ }^{\tau} \phi_{\widetilde{M}} \tau^{2} \phi_{\widetilde{M}}\left(\breve{\lambda}_{2}\left(a_{3}\right)\right)+\cdots, \\
b_{2} & =\breve{\gamma}_{1}\left(a_{2}+{ }^{2} \phi_{\widetilde{M}}\left(\breve{\lambda}_{1}\left(a_{3}\right)\right)+{ }^{\tau^{2}} \phi_{\widetilde{M}}{ }^{\tau^{3}} \phi_{\widetilde{M}}\left(\breve{\lambda}_{2}\left(a_{4}\right)\right)+\cdots\right), \ldots
\end{aligned}
$$

Pour simplifier on note $H=\tau \widetilde{f} \psi_{\widetilde{M}_{1}}-\psi_{\widetilde{M}_{2}} \widetilde{f}: \widetilde{M}_{1} \rightarrow{ }^{\tau} \widetilde{M}_{2} \otimes(\oslash \widehat{\otimes} I)$. La formule pour $\mathcal{E}_{\left(\widetilde{M}_{2}, \phi \widetilde{M}_{2}\right),\left(\widetilde{M}_{1}, \phi \widetilde{M}_{1}\right)}(f)$ s'obtient heuristiquement en notant $F$ comme dans (29), en écrivant que $\mathcal{E}_{\left(\widetilde{M}_{2}, \phi_{\widetilde{M}_{2}}\right),\left(\widetilde{M}_{1}, \phi_{\widetilde{M}_{1}}\right)}(f)$ envoie la somme (35) sur

$$
\begin{aligned}
\phi_{\widetilde{M}_{1}}{ }^{\tau} F \frac{a_{1}}{z-\pi}+ & \phi_{\widetilde{M}_{1}}{ }^{\tau} \phi_{\widetilde{M}_{1}} \tau^{2} F \frac{a_{2}}{z-\pi^{q}}+\cdots \\
= & \phi_{\widetilde{M}_{1}}\left({ }^{\tau} \widetilde{f}+{ }^{\tau} \phi_{\widetilde{M}_{2}} \frac{{ }^{\tau} H}{z-\pi^{q}}+{ }^{\tau} \phi_{\widetilde{M}_{2}}{ }^{2} \phi_{\widetilde{M}_{2}} \frac{\tau^{2} H^{\tau} \psi_{\widetilde{M}_{2}}}{\left(z-\pi^{q}\right)\left(z-\pi^{q^{2}}\right)}+\cdots\right) \frac{a_{1}}{z-\pi} \\
& +\phi_{\widetilde{M}_{1}}{ }^{\tau} \phi_{\widetilde{M}_{1}}\left(\tau^{2} \widetilde{f}+{ }^{\tau^{2}} \phi_{\widetilde{M}_{2}} \frac{\tau^{2} H}{z-\pi^{q^{2}}}+\cdots\right) \frac{a_{2}}{z-\pi^{q}}+\cdots
\end{aligned}
$$

puis en transformant $\tau^{i} H$ en $\left(\pi^{q^{i}}-\pi^{q^{i-1}}\right) \cdots\left(\pi^{q^{i}}-\pi\right) \breve{\gamma}_{i}\left(\tau^{i} H\right)$ et en utilisant les puissances de $\pi$ dans cette dernière expression pour décomposer les fractions en éléments simples de façon à obtenir une expression de la forme (35). Explicitement $\mathcal{E}_{\left(\widetilde{M}_{2}, \phi_{\widetilde{M}_{2}}\right),\left(\widetilde{M}_{1}, \phi_{\widetilde{M}_{1}}\right)}(f)$ s'obtient par passage au quotient de l'application $\bigoplus_{n \in \mathbb{N}^{*}} \tau^{n} \widetilde{M}_{1} \rightarrow \bigoplus_{n \in \mathbb{N}^{*}} \tau^{n} \widetilde{M}_{2}$ donnée par la matrice

$$
\begin{aligned}
& \left(\begin{array}{cccc}
\tau \tilde{f}+\Lambda & 0 & 0 & \cdots \\
\breve{\gamma}_{1}\left(\tau\left(H+\Lambda \psi_{\widetilde{M}_{1}}\right)\right) & \tau(\tau \widetilde{f}+\Lambda) & 0 & \ddots \\
\breve{\gamma}_{2}\left(\tau^{2}\left(H+\Lambda \psi \widetilde{M}_{1}\right)\right)^{\tau} \psi_{\widetilde{M}_{1}}{ }^{\tau}\left(\breve{\gamma}_{1}\left({ }^{\tau}\left(H+\Lambda \psi_{\widetilde{M}_{1}}\right)\right)\right) & \tau^{2}(\tau \widetilde{f}+\Lambda) & \ddots \\
\vdots & \ddots & \ddots & \ddots
\end{array}\right) \\
& \text { où } \Lambda={ }^{\tau} \phi_{\widetilde{M}_{1}} \breve{\lambda}_{1}\left({ }^{\tau} H\right)+{ }^{\tau} \phi_{\widetilde{M}_{1}} \tau^{2} \phi_{\widetilde{M}_{1}} \breve{\lambda}_{2}\left({ }^{2} H\right)^{\tau} \psi_{\widetilde{M}_{2}}+\cdots:{ }^{\tau} \widetilde{M}_{1} \rightarrow{ }^{\tau} \widetilde{M}_{2} \otimes(\theta \widehat{\otimes} I) \text {. }
\end{aligned}
$$

La commutativité des diagrammes (28) et (36) montre la compatibilité entre notre construction du $\theta$-cristal associé à un chtouca local minuscule et la construction analogue à celle 
de [48] en égales caractéristiques. Contrairement aux coefficients non-diagonaux de la matrice ci-dessus (qui utilisent vraiment les puissances divisées au sens de Grothendieck et Berthelot), le coefficient ${ }^{\tau} \widetilde{f}+\Lambda$ en haut à gauche a les mêmes dénominateurs qu'un logarithme (et n'utilise donc que les puissances divisées au sens de Honda et Gross-Hopkins). Le dual de l'algèbre de Lie de l'extension vectorielle universelle est $\mathcal{E}\left(\widetilde{M}, \phi_{\widetilde{M}}\right) / \phi_{\mathcal{E}}\left({ }^{\tau} \mathcal{E}\left(\widetilde{M}, \phi_{\widetilde{M}}\right)\right)=$ ${ }^{\tau} \widetilde{M} /(z-\pi)^{\tau} \widetilde{M}$ pour tout relèvement $\left(\widetilde{M}, \phi_{\widetilde{M}}\right)$ et l'action de $\mathcal{E}_{\left(\widetilde{M}_{2}, \phi_{\widetilde{M}_{2}}\right),\left(\widetilde{M}_{1}, \phi_{\widetilde{M}_{1}}\right)}(f)$ sur ce quotient s'obtient en évaluant $\tau \widetilde{f}+\Lambda$ en $z=\pi$. C'est aussi l'évaluation en $z=\pi$ de $^{\tau} F$ avec $F$ comme dans (29), et comme $F$ est aussi la formule pour $\mathcal{F}_{\left(\widetilde{M}_{2}, \phi \widetilde{M}_{2}\right),\left(\widetilde{M}_{1}, \phi \widetilde{M}_{1}\right)}(f)$ par (16) et (17), on retrouve d'une autre manière la compatibilité entre notre construction du $\Theta$-cristal et la construction analogue en égales caractéristiques à celle de [48].

\subsection{Un procédé de calcul plus souple pour le foncteur de Dieudonné}

Nous allons assouplir la construction du foncteur $\mathcal{F}$ de telle sorte que l'isomorphisme $\mathcal{F}\left(M, \phi_{M}\right) \simeq \widetilde{M} \otimes_{\widehat{\vartheta} \widehat{\otimes} B} \mathscr{D}_{n}(B, I)$ de (6) ait encore lieu pour des relèvements qui ne sont plus nécessairement des chtoucas locaux minuscules sur $B$, plus précisément pour les $\left(\widetilde{M}, \widetilde{\phi_{M}}\right)$ tels que $\widetilde{M}$ est un $\theta \widehat{\otimes} B$-module libre relevant $M$ et $\widehat{\phi}_{M}:{ }^{\tau} \widetilde{M} \rightarrow \widetilde{M}$ un morphisme de $\oslash \widehat{\otimes} B$-modules relevant $\phi_{M}$ (mais $\widetilde{\phi_{M}}(\widetilde{M})$ ne contient pas forcément $(z-\pi) \widetilde{M}$ ). De tels objets n'ont pas d'analogues connus en inégales caractéristiques. Cet assouplissement nous sera utile au paragraphe 10 .

Soient $B$ une $\theta$-algèbre où l'image de $\pi$ est nilpotente, $I$ un idéal de $B$ et $n \in \mathbb{N}^{*}$. Soit $f:\left(M_{1}, \phi_{M_{1}}\right) \rightarrow\left(M_{2}, \phi_{M_{2}}\right)$ un morphisme de chtoucas locaux minuscules sur $B / I$. On rappelle que si $\left(\widetilde{M}_{1}, \phi_{\widetilde{M}_{1}}\right)$ et $\left(\widetilde{M}_{1}, \phi_{\widetilde{M}_{1}}\right)$ sont des chtoucas locaux minuscules sur $B$ qui relèvent $\left(M_{1}, \phi_{M_{1}}\right)$ et $\left(M_{2}, \phi_{M_{2}}\right)$, on a défini dans (17) un morphisme de $\mathscr{D}_{n}(B, I)$-modules

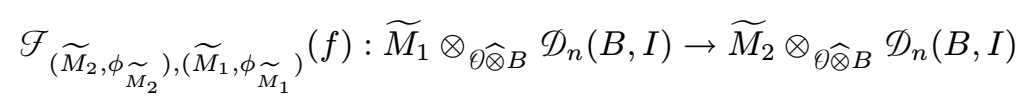

qui vérifie le lemme 5.9. Nous allons généraliser cette construction de la façon suivante. Si $\widetilde{M}_{1}$ et $\widetilde{M}_{2}$ sont des $\theta \widehat{\otimes} B$-modules libres qui relèvent $M_{1}$ et $M_{2}$ et $\widetilde{\phi_{M_{1}}}:{ }^{\tau} \widetilde{M}_{1} \rightarrow \widetilde{M}_{1}$ et $\widehat{\phi_{M_{2}}}$ des morphismes de $\theta \widehat{\otimes} B$-modules qui relèvent $\phi_{M_{1}}$ et $\phi_{M_{2}}$, nous allons définir un morphisme de $\mathscr{D}_{n}(B, I)$-modules

$$
\mathcal{F}_{\left(\widetilde{M_{2}}, \widetilde{\phi_{M_{2}}}\right),\left(\widetilde{M}_{1}, \widetilde{\phi_{M_{1}}}\right)}(f): \widetilde{M}_{1} \otimes_{\oslash \widehat{\otimes} B} \mathscr{D}_{n}(B, I) \rightarrow \widetilde{M}_{2} \otimes_{\oslash \widehat{\otimes} B} \mathscr{D}_{n}(B, I)
$$

qui vérifie des propriétés analogues à celle du lemme 5.9 et qui coïncide avec $\mathcal{F}_{\left(\widetilde{M}_{2}, \phi_{\widetilde{M}_{2}}\right),\left(\widetilde{M}_{1},{ }_{\widetilde{M}_{1}}\right)}(f)$ si $\left(\widetilde{M}_{1}, \widetilde{\phi_{M_{1}}}\right)$ et $\left(\widetilde{M}_{2}, \widetilde{\phi_{M_{2}}}\right)$ sont des chtoucas locaux sur $B$ (que l'on note alors $\left(\widetilde{M}_{1}, \phi_{\widetilde{M}_{1}}\right)$ et $\left.\left(\widetilde{M}_{2}, \phi_{\widetilde{M}_{2}}\right)\right)$.

Soient $\left(M, \phi_{M}\right)$ un chtouca local minuscule sur $B / I, \widetilde{M}$ un $\oslash \widehat{\otimes} B$-module libre relevant $M$ et $\widehat{\phi_{M}}$ un relèvement de $\phi_{M}$. Nous commençons par construire un morphisme de $\mathscr{D}_{n}(B, I)$-modules

$$
\left((z-\pi){\widetilde{\phi_{M}}}^{-1}\right): \widetilde{M} \otimes_{\partial \widehat{\otimes} \mathcal{B}} \mathscr{D}_{n}(B, I) \rightarrow{ }^{\tau} \widetilde{M} \otimes_{\partial \widehat{\otimes} \mathcal{B}} \mathscr{D}_{n}(B, I)
$$

qui coïncide avec $\psi_{\widetilde{M}}$ si $\left(\widetilde{M}, \widetilde{\phi_{M}}\right)=\left(\widetilde{M}, \phi_{\widetilde{M}}\right)$ est un chtouca local minuscule sur $B$. 
Soit $\widetilde{\psi_{M}}: \widetilde{M} \rightarrow \tau \widetilde{M}$ un relèvement de $\psi_{M}$. On a

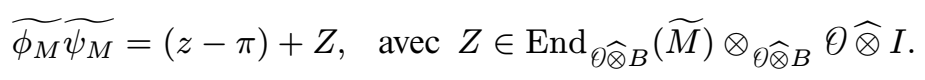

Or $\quad(z-\pi)+Z=(z-\pi)\left(1+\check{\epsilon}_{0}(Z)\right)$ et $1+\check{\epsilon}_{0}(Z) \quad$ est inversible dans $\operatorname{End}_{\mathscr{D}_{n}(B, I)}\left(\widetilde{M} \otimes_{\vartheta \widehat{\otimes} B} \mathscr{D}_{n}(B, I)\right)$ (car, dans une base de $\widetilde{M}, \check{\epsilon}_{0}(Z)$ est une matrice à coefficients nilpotents dans $\left.\mathscr{D}_{n}(B, I)\right)$. On pose

$$
\theta=\widetilde{\psi_{M}}\left(1+\check{\epsilon}_{0}(Z)\right)^{-1}
$$

On voit que $\widetilde{\phi_{M}} \theta=(z-\pi) \operatorname{Id}_{\widetilde{M} \otimes \widehat{\vartheta \otimes B}} \mathscr{D}_{n}(B, I)$, mais cette relation ne détermine pas en général $\theta$ de manière unique. En effet dans les notations de l'exemple 5.7, en prenant $\left(\widetilde{M}, \widetilde{\phi_{M}}\right)=$ $(\theta \widehat{\otimes} B, z-\pi)$ et en choisissant $\widetilde{\psi_{M}}=1$ pour le calcul on voit que $\theta=1$, mais que $X=1+\epsilon(\pi) \epsilon(x)$ vérifie aussi la relation $\widetilde{\phi_{M}} X=(z-\pi) \operatorname{Id}_{\widetilde{M} \otimes_{\widehat{\otimes \otimes \mathscr{B}}} \mathscr{D}_{n}(B, I)}$. Cependant le lemme suivant montre que $\theta$ a de bonnes propriétés.

Lemme 5.11. - Le morphisme de $\mathscr{D}_{n}(B, I)$-modules

$$
\theta: \widetilde{M} \otimes_{\partial \widehat{\otimes} \mathscr{B}} \mathscr{D}_{n}(B, I) \rightarrow{ }^{\tau} \widetilde{M} \otimes_{\partial \widehat{\otimes} \mathscr{B}} \mathscr{D}_{n}(B, I)
$$

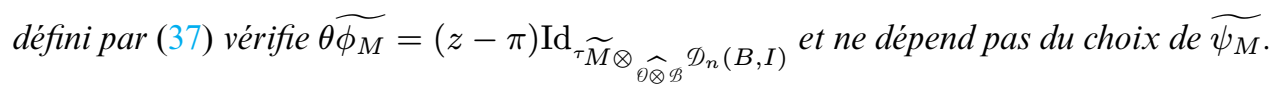

Démonstration. - Soit $Z^{\prime} \in \operatorname{End}_{\widehat{\partial} \widehat{\otimes} B}\left({ }^{\tau} \widetilde{M}\right) \otimes_{\vartheta \widehat{\otimes} B} \oslash \widehat{\otimes} I$ défini par $\widetilde{\psi_{M} \widetilde{\phi_{M}}}=(z-\pi)+Z^{\prime}$. En écrivant $\widetilde{\psi_{M}} \widetilde{\phi_{M}} \widetilde{\psi_{M}}$ et $\widetilde{\phi_{M}} \widetilde{\psi_{M}} \widetilde{\phi_{M}}$ de deux manières différentes on voit que

$$
\begin{aligned}
Z^{\prime} \widetilde{\psi_{M}} & =\widetilde{\psi_{M}} Z \text { dans } \operatorname{Hom}_{\partial \widehat{\otimes} B}\left(\widetilde{M},{ }^{\tau} \widetilde{M}\right) \otimes_{\partial \widehat{\otimes} B} \theta \widehat{\otimes} I \\
\text { et } \widetilde{\phi_{M}} Z^{\prime} & =Z \widetilde{\phi_{M}} \text { dans } \operatorname{Hom}_{\partial \widehat{\otimes} B}\left({ }^{\tau} \widetilde{M}, \widetilde{M}\right) \otimes_{\partial \widehat{\otimes} B} \theta \widehat{\otimes} I .
\end{aligned}
$$

Comme $Z^{\prime} \in \operatorname{End}_{\partial \widehat{\otimes} B}\left({ }^{\tau} \widetilde{M}\right) \otimes_{\vartheta \widehat{\otimes} B} \oslash \widehat{\otimes} I, 1+\check{\epsilon}_{0}\left(Z^{\prime}\right)$ admet un inverse

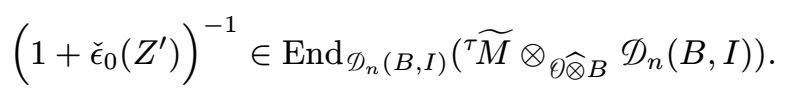

On a

$$
\theta=\left(1+\check{\epsilon}_{0}\left(Z^{\prime}\right)\right)^{-1} \widetilde{\psi_{M}}
$$

En effet cela équivaut à

$$
\widetilde{\psi_{M}}\left(1+\check{\epsilon}_{0}(Z)\right)=\left(1+\check{\epsilon}_{0}\left(Z^{\prime}\right)\right) \widetilde{\psi_{M}}
$$

qui découle de (38) puisque $\widetilde{\psi_{M}} \check{\epsilon}_{0}(Z)=\check{\epsilon}_{0}\left(\widetilde{\psi_{M}} Z\right)$ et $\check{\epsilon}_{0}\left(Z^{\prime}\right) \widetilde{\psi_{M}}=\check{\epsilon}_{0}\left(Z^{\prime} \widetilde{\psi_{M}}\right)$.

L'égalité $\theta \widetilde{\phi_{M}}=(z-\pi) \operatorname{Id}_{\tau \widetilde{M} \otimes_{\widehat{Q \otimes \mathscr{B}}} \Phi_{n}(B, I)}$ qui fait partie de l'énoncé du lemme résulte de (40) et du fait que

$$
\widetilde{\psi_{M}} \widetilde{\phi_{M}}=(z-\pi)+Z^{\prime}=(z-\pi)\left(1+\check{\epsilon}_{0}\left(Z^{\prime}\right)\right) \text {. }
$$

Soient maintenant $\widetilde{\psi_{M}}{ }^{\sharp}$ un autre relèvement de $\psi_{M}$, et $Z^{\sharp}, Z^{\prime \sharp}$ et $\theta^{\sharp}$ construits à partir de $\widetilde{\psi_{M}}{ }^{\sharp}$ comme $Z, Z^{\prime}$ et $\theta$ à partir de $\widetilde{\psi_{M}}$. On veut montrer $\theta^{\sharp}=\theta$. On a $\widetilde{\psi_{M}}{ }^{\sharp}=\widetilde{\psi_{M}}+K$ avec 


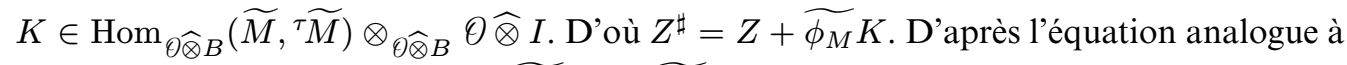
(37) pour $\theta^{\sharp}$ et grâce à l'égalité $\check{\epsilon}_{0}\left(\widetilde{\phi_{M}} K\right)=\widetilde{\phi_{M}} \check{\epsilon}_{0}(K)$, on a

$$
\theta^{\sharp}=\left(\widetilde{\psi_{M}}+K\right)\left(1+\check{\epsilon}_{0}(Z)+\widetilde{\phi_{M}} \check{\epsilon}_{0}(K)\right)^{-1} .
$$

Grâce à (40) on est ramené à montrer

$$
\left(\widetilde{\psi_{M}}+K\right)\left(1+\check{\epsilon}_{0}(Z)+\widetilde{\phi_{M}} \check{\epsilon}_{0}(K)\right)^{-1}=\left(1+\check{\epsilon}_{0}\left(Z^{\prime}\right)\right)^{-1} \widetilde{\psi_{M}}
$$

ce qui équivaut à

$$
\left(1+\check{\epsilon}_{0}\left(Z^{\prime}\right)\right)\left(\widetilde{\psi_{M}}+K\right)=\widetilde{\psi_{M}}\left(1+\check{\epsilon}_{0}(Z)+\widetilde{\phi_{M}} \check{\epsilon}_{0}(K)\right) .
$$

Cette égalité découle de (41) et du calcul suivant :

$$
\left(1+\check{\epsilon}_{0}\left(Z^{\prime}\right)\right) K=\left(1+\check{\epsilon}_{0}\left(Z^{\prime}\right)\right)(z-\pi) \check{\epsilon}_{0}(K)=\left(z-\pi+Z^{\prime}\right) \check{\epsilon}_{0}(K)=\widetilde{\psi_{M}} \widetilde{\phi_{M}} \check{\epsilon}_{0}(K) .
$$

Comme $\theta$ ne dépend pas du choix de $\widetilde{\psi_{M}}$ on notera

$$
\left((z-\pi){\widetilde{\phi_{M}}}^{-1}\right): \widetilde{M} \otimes_{\vartheta \widehat{\otimes} \mathscr{B}} \mathscr{D}_{n}(B, I) \rightarrow{ }^{\tau} \widetilde{M} \otimes_{\vartheta \widehat{\otimes} \mathcal{B}} \mathscr{D}_{n}(B, I)
$$

ce morphisme $\theta$. On a

$$
\widetilde{\phi_{M}}\left((z-\pi){\widetilde{\phi_{M}}}^{-1}\right)=(z-\pi) \text { et }\left((z-\pi){\widetilde{\phi_{M}}}^{-1}\right) \widetilde{\phi_{M}}=(z-\pi) \text {. }
$$

Plus loin, dans le lemme 5.13 nous aurons besoin d'une autre relation satisfaite par $\left((z-\pi){\widetilde{\phi_{M}}}^{-1}\right)$ et nous devrons revenir aux formules (37) et (40) pour la démontrer.

Soit $\tilde{f}: \widetilde{M_{1}} \rightarrow \widetilde{M_{2}}$ un morphisme de $\theta \widehat{\otimes} B$-modules qui relève $f$. Nous allons maintenant définir $\mathcal{F}_{\left(\widetilde{M_{2}}, \widetilde{\phi_{M_{2}}}\right),\left(\widetilde{M_{1}}, \widetilde{\phi_{M_{1}}}\right)}(\widetilde{f})$ en reprenant les formules (15) et (17) et en effectuant les remplacements

$$
\text { de } \phi_{\widetilde{M}_{i}} \operatorname{par} \widetilde{\phi_{M_{i}}} \text { et de } \psi_{\widetilde{M}_{i}} \operatorname{par}\left((z-\pi){\widetilde{\phi_{M_{i}}}}^{-1}\right)
$$

pour tout $i$. Comme $\left((z-\pi){\widetilde{\phi_{M_{i}}}}^{-1}\right)$ est à coefficients dans $\mathscr{D}_{n}(B, I)$, la remarque suivante montre que les formules (14) et (16) n'ont plus de sens, alors que (15) garde un sens lorsqu'on effectue les remplacements (42). Nous devrons donc modifier les arguments qui utilisaient les formules (14) et (16).

Remarque. - Soit $\mathscr{I}$ l'idéal de $\mathscr{D}_{n}(B, I)$ engendré par $\theta \widehat{\otimes} I$. Il n'est pas vrai en général que $\check{\epsilon}_{0}$ s'étende en un morphisme $\mathscr{D}_{n}(B, I)$-linéaire de $\mathscr{I}$ dans $\mathscr{D}_{n}(B, I)$. En effet pour $x_{1}, \ldots, x_{k}, y_{1}, \ldots, y_{k} \in I$ on devrait avoir $\check{\epsilon}_{0}\left(\sum_{i=1}^{k} x_{i} \epsilon\left(y_{i}\right)\right)=\sum_{i=1}^{k} \epsilon_{0}\left(x_{i}\right) \epsilon\left(y_{i}\right)$ mais $\sum_{i=1}^{k} x_{i} \epsilon\left(y_{i}\right)=\epsilon\left(\sum_{i=1}^{k} x_{i} y_{i}\right)$ et $\sum_{i=1}^{k} x_{i} y_{i}$ peut être nul sans que $\sum_{i=1}^{k} \epsilon_{0}\left(x_{i}\right) \epsilon\left(y_{i}\right)$ le soit, comme on l'a vu dans l'exemple 5.7 avec $k=1, x_{1}=\pi$ et $y_{1}=x$.

On pose donc $\mathcal{F}_{\left(\widetilde{M_{2}}, \widetilde{\phi_{M_{2}}}\right),\left(\widetilde{M_{1}}, \widetilde{\phi_{M_{1}}}\right)}(\widetilde{f})=\widetilde{f}$ et pour $m \in \mathbb{N}^{*}$,

$$
\begin{aligned}
& \mathcal{F}_{\left(\widetilde{M_{2}}, \widetilde{\phi_{M_{2}}}\right),\left(\widetilde{M_{1}}, \widetilde{\phi_{M_{1}}}\right)}^{m}(\widetilde{f})=\widetilde{\phi_{M_{2}}} \ldots \tau^{m-2}\left(\widetilde{\phi_{M_{2}}}\right) \check{\epsilon}_{m-1}\left(\tau^{m-1}\left(\widetilde{\phi_{M_{2}}} \tau \widetilde{f}-\widetilde{f \phi_{M_{1}}}\right)\right) \\
& \tau^{m-1}\left((z-\pi){\widetilde{\phi_{M_{1}}}}^{-1}\right) \cdots\left((z-\pi){\widetilde{\phi_{M_{1}}}}^{-1}\right) \in \operatorname{Hom}_{\emptyset \widehat{\otimes} B}\left(\widetilde{M_{1}}, \widetilde{M_{2}}\right) \otimes_{\emptyset \widehat{\otimes} B} \mathscr{D}_{n}(B, I) .
\end{aligned}
$$


Comme dans (17) on pose

$$
\mathcal{F}_{\left(\widetilde{M_{2}}, \widetilde{\phi_{M_{2}}}\right),\left(\widetilde{M_{1}}, \widetilde{\phi_{M_{1}}}\right)}(\widetilde{f})=\sum_{m=0}^{n} \mathcal{F}_{\left(\widetilde{M_{2}}, \widetilde{\phi_{M_{2}}}\right),\left(\widetilde{M_{1}}, \widetilde{\phi_{M_{1}}}\right)}^{m}(\widetilde{f}) .
$$

En recopiant la démonstration du lemme 5.8 (avec les remplacements (42)) on montre que $\mathcal{F}\left(\widetilde{M_{2}}, \widetilde{\phi_{M_{2}}}\right),\left(\widetilde{M_{1}}, \widetilde{\left.\phi_{M_{1}}\right)}(\widetilde{f})\right.$ ne dépend pas du choix du relèvement $\widetilde{f}$ et on le note donc $\mathcal{F}_{\left.\left(\widetilde{M_{2}}, \widetilde{\phi_{M_{2}}}\right), \widetilde{M_{1}}, \widetilde{\phi_{M_{1}}}\right)}(f)$.

Lemme 5.12. - On a

$$
\widetilde{\phi_{M_{2}}} \tau \mathcal{F}\left(\widetilde{M_{2}}, \widetilde{\phi_{M_{2}}}\right),\left(\widetilde{M_{1}}, \widetilde{\phi_{M_{1}}}\right)(f)=\mathcal{F}_{\left(\widetilde{M_{2}}, \widetilde{\phi_{M_{2}}}\right),\left(\widetilde{M_{1}}, \widetilde{\phi_{M_{1}}}\right)}(f) \widetilde{\phi_{M_{1}}}
$$

et

$$
\tau \mathcal{F}_{\left(\widetilde{M_{2}}, \widetilde{\phi_{M_{2}}}\right),\left(\widetilde{M_{1}}, \widetilde{\left.\phi_{M_{1}}\right)}\right.}(f)\left((z-\pi){\widetilde{\phi_{M_{1}}}}^{-1}\right)=\left((z-\pi){\widetilde{\phi_{M_{2}}}}^{-1}\right) \mathcal{F}_{\left(\widetilde{M_{2}}, \widetilde{\phi_{M_{2}}}\right),\left(\widetilde{M_{1}}, \widetilde{\phi_{M_{1}}}\right)}(f) .
$$

De plus la réduction modulo $\mathcal{J}_{n} d e \mathcal{F}_{\left(\widetilde{M_{2}}, \widetilde{\phi_{M_{2}}}\right),\left(\widetilde{M_{1}}, \widetilde{\left.\phi_{M_{1}}\right)}\right.}(f)$ est égale à $f \otimes_{0 \widehat{\otimes}(B / I)} \operatorname{Id}_{\mathscr{D}_{n}(B, I) / \mathscr{f}_{n}}$. Enfin, si $\left(M_{3}, \phi_{M_{3}}\right)$ est un troisième chtouca local minuscule sur $(B / I), \widetilde{M_{3}}$ un $\oslash \widehat{\otimes} B$-module libre relevant $M_{3}, \widetilde{\phi_{M_{3}}}$ un relèvement de $\phi_{M_{3}}$, et $g$ un morphisme de chtoucas locaux de $\left(M_{2}, \phi_{M_{2}}\right)$ vers $\left(M_{3}, \phi_{M_{3}}\right)$, on a

$$
\mathcal{F}_{\left(\widetilde{M_{3}}, \widetilde{\phi_{M_{3}}}\right),\left(\widetilde{M_{2}}, \widetilde{\left.\phi_{M_{2}}\right)}\right.}(g) \mathcal{F}_{\left(\widetilde{M_{2}}, \widetilde{\phi_{M_{2}}}\right),\left(\widetilde{M_{1}}, \widetilde{\left.\phi_{M_{1}}\right)}\right.}(f)=\mathcal{F}_{\left(\widetilde{M_{3}}, \widetilde{\phi_{M_{3}}}\right),\left(\widetilde{M_{1}}, \widetilde{\left.\phi_{M_{1}}\right)}\right.}(g \circ f) .
$$

Démonstration. - Soient $\tilde{f}$ et $\widetilde{g}$ des relèvements de $f$ et $g$. Pour montrer (43) on recopie la preuve de (19) (avec les remplacements (42)). On obtient également que pour tout $m \in \mathbb{N}$

$$
\widetilde{\phi_{M_{2}}} \tau\left(\sum_{l=0}^{m} \mathcal{F}_{\left(\widetilde{M_{2}}, \widetilde{\phi_{M_{2}}}\right),\left(\widetilde{M_{1}}, \widetilde{\phi_{M_{1}}}\right)}^{l}(\widetilde{f})\right)=\left(\sum_{l=0}^{m+1} \mathcal{F}_{\left(\widetilde{M}_{2}, \widetilde{\phi_{M_{2}}}\right),\left(\widetilde{M_{1}}, \widetilde{\left.\phi_{M_{1}}\right)}\right.}^{l}(\tilde{f})\right) \widetilde{\phi_{M_{1}}} \text {. }
$$

Pour montrer (44) on commence par un lemme.

Lemme 5.13. - On a

$$
\begin{aligned}
\left((z-\pi){\widetilde{\phi_{M_{2}}}}^{-1}\right) \check{\epsilon}_{0}\left({\widetilde{\phi_{M_{2}}}}^{\tau} \widetilde{f}-\widetilde{f \phi_{M_{1}}}\right)\left((z-\pi){\widetilde{\phi_{M_{1}}}}^{-1}\right) \\
\quad={ }^{\tau} \widetilde{f}\left((z-\pi){\widetilde{\phi_{M_{1}}}}^{-1}\right)-\left((z-\pi){\widetilde{\phi_{M_{2}}}}^{-1}\right) \widetilde{f}
\end{aligned}
$$

REMARQUe. - Le lemme serait évident si on pouvait faire rentrer $\left((z-\pi){\widetilde{\phi_{M_{2}}}}^{-1}\right)$ et $\left((z-\pi){\widetilde{\phi_{M_{1}}}}^{-1}\right)$ dans l'expression centrale à laquelle on applique $\check{\epsilon}_{0}$ mais la remarque avant le lemme 5.12 montre que ce n'est pas possible.

Démonstration. - Soient $\widetilde{\psi_{M_{1}}}$ et $\widetilde{\psi_{M_{2}}}$ des relèvements de $\psi_{M_{1}}$ et $\psi_{M_{2}}$. En définissant $Z_{1}$ $\operatorname{par} \widetilde{\phi_{M_{1}}} \widetilde{\psi_{M_{1}}}=z-\pi+Z_{1}$ et $Z_{2}^{\prime}$ par $\widetilde{\psi_{M_{2}}} \widetilde{\phi_{M_{2}}}=z-\pi+Z_{2}^{\prime}$ on a, grâce à (37) et (40),

$$
\begin{aligned}
\left((z-\pi){\widetilde{\phi_{M_{2}}}}^{-1}\right) \check{\epsilon}_{0}\left(\widetilde{\phi_{M_{2}}} \tau \tilde{f}-\widetilde{f \phi_{M_{1}}}\right)\left((z-\pi){\widetilde{\phi_{M_{1}}}}^{-1}\right) \\
=\left(1+\check{\epsilon}_{0}\left(Z_{2}^{\prime}\right)\right)^{-1} \widetilde{\psi_{M_{2}} \check{\epsilon}_{0}}\left(\widetilde{\phi_{M_{2}}} \tau \widetilde{f}-\widetilde{f \phi_{M_{1}}}\right) \widetilde{\psi_{M_{1}}}\left(1+\check{\epsilon}_{0}\left(Z_{1}\right)\right)^{-1}
\end{aligned}
$$


et ceci est égal à $\tau \widetilde{f}\left((z-\pi){\widetilde{\phi_{M_{1}}}}^{-1}\right)-\left((z-\pi){\widetilde{\phi_{M_{2}}}}^{-1}\right) \tilde{f}$ car

$$
\begin{aligned}
& \left(1+\check{\epsilon}_{0}\left(Z_{2}^{\prime}\right)\right)\left(\tau \tilde{f}\left((z-\pi){\widetilde{\phi_{M_{1}}}}^{-1}\right)-\left((z-\pi){\widetilde{\phi_{M_{2}}}}^{-1}\right) \tilde{f}\right)\left(1+\check{\epsilon}_{0}\left(Z_{1}\right)\right) \\
& =\left(1+\check{\epsilon}_{0}\left(Z_{2}^{\prime}\right)\right)^{\tau} \widetilde{f} \psi_{M_{1}}-\widetilde{\psi_{M_{2}}} \widetilde{f}\left(1+\check{\epsilon}_{0}\left(Z_{1}\right)\right) \\
& \left.=(z-\pi) \check{\epsilon}_{0}\left(\tau \widetilde{f \psi_{M_{1}}}-\widetilde{\psi_{M_{2}}} \widetilde{f}\right)+\check{\epsilon}_{0}\left(Z_{2}^{\prime} \tau \widetilde{f \psi_{M_{1}}}\right)-\check{\epsilon}_{0}\left(\widetilde{\psi_{M_{2}}} \widetilde{f} Z_{1}\right)\right) \\
& =\check{\epsilon}_{0}\left(\left(z-\pi+Z_{2}^{\prime}\right)^{\tau} \widetilde{f} \psi_{M_{1}}-\widetilde{\psi_{M_{2}}} \widetilde{f}\left(z-\pi+Z_{1}\right)\right) \\
& =\check{\epsilon}_{0}\left(\widetilde{\psi_{M_{2}}} \widetilde{\phi_{M_{2}}} \tau \widetilde{f \psi_{M_{1}}}-\widetilde{\psi_{M_{2}}} \widetilde{f \phi_{M_{1}}} \widetilde{\psi_{M_{1}}}\right)=\widetilde{\psi_{M_{2}} \check{\epsilon}_{0}}\left(\widetilde{\phi_{M_{2}}} \tau \widetilde{f}-\widetilde{f \phi_{M_{1}}}\right) \widetilde{\psi_{M_{1}}} \text {. }
\end{aligned}
$$

Suite de la démonstration du lemme 5.12. - Il résulte immédiatement du lemme 5.13 que

$$
\begin{aligned}
& \left.\tau \mathcal{F}_{\left(\widetilde{M_{2}}\right.}^{0}, \widetilde{\phi_{M_{2}}}\right),\left(\widetilde{M_{1}}, \widetilde{\phi_{M_{1}}}\right)(\widetilde{f})\left((z-\pi) \widetilde{\phi_{M_{1}}}-1\right) \\
& =\left((z-\pi){\widetilde{\phi_{M_{1}}}}^{-1}\right)\left(\mathcal{F}_{\left(\widetilde{M_{2}}, \widetilde{\phi_{M_{2}}}\right),\left(\widetilde{M_{1}}, \widetilde{\phi_{M_{1}}}\right)}^{0}(\tilde{f})+\mathcal{F}_{\left(\widetilde{M_{2}}, \widetilde{\phi_{M_{2}}}\right),\left(\widetilde{M_{1}}, \widetilde{\phi_{M_{1}}}\right)}^{1}(\widetilde{f})\right) .
\end{aligned}
$$

En appliquant (12) on obtient

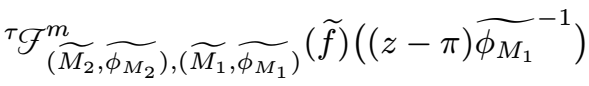

$$
\begin{aligned}
& =\widetilde{\tau} \bar{\phi}_{M_{2}} \ldots \tau^{m-1}\left(\widetilde{\phi_{M_{2}}}\right)^{\tau}\left(\check{\epsilon}_{m-1}\left(\tau^{m-1}\left(\widetilde{\phi_{M_{2}}} \tau \widetilde{f}-\widetilde{f \phi_{M_{1}}}\right)\right)\right) \\
& \tau^{m}\left((z-\pi){\widetilde{\phi_{M_{1}}}}^{-1}\right) \cdots\left((z-\pi){\widetilde{\phi_{M_{1}}}}^{-1}\right) \\
& =(z-\pi)^{\tau} \widetilde{\phi_{M_{2}}} \ldots \tau^{m-1}\left(\widetilde{\phi_{M_{2}}}\right) \check{\epsilon}_{m}\left(\tau^{m}\left(\widetilde{\phi_{M_{2}}} \tau \tilde{f}-\widetilde{f \phi_{M_{1}}}\right)\right) \\
& \tau^{m}\left((z-\pi){\widetilde{\phi_{M_{1}}}}^{-1}\right) \cdots\left((z-\pi){\widetilde{\phi_{M_{1}}}}^{-1}\right) \\
& =\left((z-\pi){\widetilde{\phi_{M_{1}}}}^{-1}\right) \mathcal{F}_{\left(\widetilde{M_{2}}, \widetilde{\phi_{M_{2}}}\right),\left(\widetilde{M_{1}}, \widetilde{\phi_{M_{1}}}\right)}^{\widetilde{c}}(\widetilde{f}) \text {. }
\end{aligned}
$$

Il résulte des deux suites d'égalités précédentes que pour tout $m \in \mathbb{N}$ on a

$$
\begin{aligned}
\tau\left(\sum_{l=0}^{m} \mathcal{F}_{\left(\widetilde{M_{2}}, \widetilde{\phi_{M_{2}}}\right),\left(\widetilde{M_{1}}, \widetilde{\phi_{M_{1}}}\right)}^{l}(\tilde{f})\right)\left((z-\pi){\widetilde{\phi_{M_{1}}}}^{-1}\right) \\
=\left((z-\pi){\widetilde{\phi_{M_{1}}}}^{-1}\right)\left(\sum_{l=0}^{m+1} \mathcal{F}_{\left.\left(\widetilde{M_{2}}, \widetilde{\phi_{M_{2}}}\right), \widetilde{M_{1}}, \widetilde{\phi_{M_{1}}}\right)}^{(\widetilde{f})) .}\right.
\end{aligned}
$$

L'équation (44) en résulte immédiatement en prenant $m=n$.

La deuxième assertion est évidente. Pour montrer la dernière assertion on reprend les calculs du lemme 5.9 (avec les remplacements (42)) : (45) résulte de (46) et (47) de la même façon que (21) résultait de (22) et (23).

La construction que nous venons d'effectuer généralise celle du foncteur $\mathcal{F}$ dans la démonstration de la proposition 5.6. Pour pouvoir y référer ultérieurement on va en énoncer une conséquence.

Proposition 5.14. - Si $\left(M, \phi_{M}\right)$ est un chtouca local minuscule sur $B / I$ et si $\widetilde{M}$ est un $\vartheta \widehat{\otimes} B$-module libre relevant $M$ et $\widetilde{\phi_{M}}$ est un relèvement de $\phi_{M}, \mathcal{F}\left(M, \phi_{M}\right)$ est canoniquement isomorphe à $\widetilde{M} \otimes_{\vartheta \widehat{\otimes} B} \mathscr{D}_{n}(B, I)$. 


\section{Le théorème de relèvement de Grothendieck}

Nous allons montrer l'analogue en égales caractéristiques du théorème de relèvement de Grothendieck [28, 48]. Ce sont les puissances divisées de Grothendieck et Berthelot [4, 9, 48] (avec la condition supplémentaire de nilpotence) qui apparaissent ici parce que l'on doit itérer $\gamma$. La définition que nous allons donner pour le $\theta$-analogue des puissances divisées de Grothendieck et Berthelot est un cas particulier de la définition 14 de [21] (Faltings considère dans cet article le cas général de l'anneau d'entiers $\theta$ d'un corps local et nous restreignons sa définition au cas où $\theta$ est d'égales caractéristiques).

Soient $B$ une $\theta$-algèbre et $I$ un idéal de $B$.

Définition 6.1. - Une structure de puissances divisées au sens de Grothendieck et Berthelot sur I est une application $\gamma: I \rightarrow I$ qui vérifie

(a) $\pi \gamma(x)=x^{q}$ pour $x \in I$,

(b) $\gamma(b x)=b^{q} \gamma(x)$ pour $x \in I$ et $b \in B$,

(c) $\gamma(x+y)=\gamma(x)+\gamma(y)$ pour $x, y \in I$.

On note $\gamma^{n}$ l'itéré $n$ fois de $\gamma$ et on note $I^{[n]}$ l'idéal de $B$ engendré par tous les produits $\gamma^{a_{1}}\left(x_{1}\right) \cdots \gamma^{a_{k}}\left(x_{k}\right)$ pour $k \in \mathbb{N}^{*}, a_{1}, \ldots, a_{k} \in \mathbb{N}^{*}$ vérifiant $\sum_{i=1}^{k} q^{a_{i}} \geq n$ et $x_{1}, \ldots, x_{k} \in I$.

On dit que $\gamma$ est nilpotent s'il existe un entier $n$ tel que $I^{[n]}=0$.

Remarque. - De nouveau on remarque que, dans la définition précédente, si $\pi \in I$ on n'a pas nécessairement $\gamma(\pi)=\pi^{q-1}$. De même pour $k \in \mathbb{N}^{*}$, et $x_{1}, \ldots, x_{k}, y_{1}, \ldots, y_{k} \in I$ tels que $\sum_{i=1}^{k} x_{i} y_{i}=0$, on n'a pas nécessairement $\sum_{i=1}^{k} \gamma\left(x_{i}\right) \gamma\left(y_{i}\right)=0$. On renvoie à l'exemple 5.7 ci-dessus pour un cas où ces égalités n'ont pas lieu.

Le lemme suivant indique le lien logique entre les puissances divisées au sens de Grothendieck et Berthelot et celles au sens de Honda et Gross-Hopkins considérées dans le paragraphe précédent.

Lemme 6.2. - a) Soient B, I, $\gamma$ comme dans la définition 6.1. Alors $(B, \mathrm{Id}, \gamma)$ est une structure de puissances divisées sur I au sens de Honda et de Gross-Hopkins comme dans la définition 5.1.

b) Supposons que l'image de $\pi$ dans $B$ est nilpotente. Soit $A$ une O-algèbre complète pour la topologie $\pi$-adique et $s: A \rightarrow B / I$ un morphisme de $\theta$-algèbres. Alors $\left(B, I, s, B, \operatorname{Id}_{B}, \gamma\right)$ est un A-objet test au sens de la définition 5.2.

Démonstration. - Seul b) demande une démonstration. Soient $n$ tel que $\pi^{n}=0$ dans $B$. Alors pour tout $x \in I$, on a $x^{q n}=\pi^{n} \gamma(x)^{n}=0$. A fortiori, comme $\gamma$ prend ses valeurs dans $I$, on a $\gamma(x)^{q n}=0$ pour tout $x \in I$, et donc la dernière condition de la définition 5.2 est satisfaite. 
Soient $B, I, \gamma$ comme dans la définition 6.1. On suppose que l'image de $\pi$ dans $B$ est nilpotente. Grâce au lemme précédent, $\left(B, I, \operatorname{Id}_{B / I}, B, \operatorname{Id}_{B}, \gamma\right)$ est un $B / I$-objet test. $\mathrm{Si}$ $\left(M, \phi_{M}\right)$ est un chtouca local minuscule sur $B / I$, nous noterons $\mathbb{D}\left(M, \phi_{M}\right)_{B}$ le $B$-module localement libre qui est la valeur du $\theta$-cristal associé à $\left(M, \phi_{M}\right)$ en le $B / I$-objet test $\left(B, I, \operatorname{Id}_{B / I}, B, \operatorname{Id}_{B}, \gamma\right)$, et $\mathbb{D}\left(M, \phi_{M}\right)_{B / I}$ la valeur du $\theta$-cristal associé à $\left(M, \phi_{M}\right)$ en le $B / I$-objet test $\left(B / I, 0, \operatorname{Id}_{B / I}, B / I, \operatorname{Id}_{B / I}, 0\right)$, qui est simplement le $B / I$-module localement libre ${ }^{\tau} M /(z-\pi)^{\tau} M$. On a

$$
\mathbb{D}\left(M, \phi_{M}\right)_{B / I}=\mathbb{D}\left(M, \phi_{M}\right)_{B} \otimes_{B} B / I .
$$

Le $B / I$-module localement libre ${ }^{\tau} M /(z-\pi)^{\tau} M$ contient le sous-module $\psi_{M}(M) /(z-\pi)^{\tau} M$ qui est facteur direct par le a) du lemme 1.1 (on rappelle que $\psi_{M}=(z-\pi) \phi_{M}^{-1}: M \rightarrow{ }^{\tau} M$ ). Si $\left(\hat{M}, \phi_{\hat{M}}\right)$ est un chtouca local minuscule sur $B$ qui relève $\left(M, \phi_{M}\right)$, le $B$-module localement libre ${ }^{\tau} \hat{M} /(z-\pi)^{\tau} \hat{M}$ est canoniquement isomorphe à $\mathbb{D}\left(M, \phi_{M}\right)_{B}$. Mais ${ }^{\tau} \hat{M} /(z-\pi)^{\tau} \hat{M}$ possède le sous- $B$-module $\psi_{\hat{M}}(\hat{M}) /(z-\pi)^{\tau} \hat{M}$ qui est facteur direct par le a) du lemme 1.1 et qui relève $\psi_{M}(M) /(z-\pi)^{\tau} M$.

Proposition 6.3. - Soit B une O-algèbre où l'image de $\pi$ est nilpotente. Soient I un idéal de $B$ et $\gamma$ une structure de puissances divisées sur $I$ au sens de la définition 6.1. On suppose $\gamma$ nilpotent. Alors le foncteur $\left(\hat{M}, \phi_{\hat{M}}\right) \mapsto\left(\left(M, \phi_{M}\right), \psi_{\hat{M}}(\hat{M}) /(z-\pi)^{\tau} \hat{M}\right)$, qui à un chtouca local minuscule $\left(\hat{M}, \phi_{\hat{M}}\right)$ sur $B$ associe sa restriction $\left(M, \phi_{M}\right)$ à $B / I$ et $\psi_{\hat{M}}(\hat{M}) /(z-\pi)^{\tau} \hat{M}$ est une équivalence entre la catégorie des chtoucas locaux minuscules sur $B$ et la catégorie des couples $\left(\left(M, \phi_{M}\right)\right.$, Fil $)$, où $\left(M, \phi_{M}\right)$ est un chtouca local minuscule sur $B / I$, et Fil est un sous- $B$-module facteur direct de $\mathbb{D}\left(M, \phi_{M}\right)_{B}$ qui relève

$$
\psi_{M}(M) /(z-\pi)^{\tau} M \subset{ }^{\tau} M /(z-\pi)^{\tau} M=\mathbb{D}\left(M, \phi_{M}\right)_{B / I}=\mathbb{D}\left(M, \phi_{M}\right)_{B} \otimes_{B} B / I .
$$

Démonstration. - Comme les images itérées de $I$ par l'application qui à un idéal $J$ de $B$ associe $\gamma(J) B$ sont nulles après un nombre fini d'itérations, il suffit de montrer la proposition sous l'hypothèse supplémentaire $\gamma(I)=0$. Dans ce cas, la proposition est impliquée par le lemme 6.4. On note que $\gamma(I)=0$ implique $I^{(q)}=0$, c'est-à-dire $x^{q}=0$ pour tout $x \in I$.

Lemme 6.4. - Soient B une O-algèbre où l'image de $\pi$ est nilpotente et I un idéal de $B$ tel que $I^{(q)}=0$. On note $\alpha: B / I \rightarrow B$ le morphisme $x \mapsto x^{q}$. Le foncteur $\left(\hat{M}, \phi_{\hat{M}}\right) \mapsto\left(\left(M, \phi_{M}\right), \psi_{\hat{M}}(\hat{M}) /(z-\pi) \alpha^{*} M\right)$ qui à un chtouca local minuscule $\left(\hat{M}, \phi_{\hat{M}}\right)$ sur $B$ associe sa réduction $\left(M, \phi_{M}\right)$ modulo $I$ et $\psi_{\hat{M}}(\hat{M}) /(z-\pi) \alpha^{*} M$ réalise une équivalence entre la catégorie des chtoucas locaux minuscules sur $B$ et la catégorie des couples $\left(\left(M, \phi_{M}\right)\right.$, Fil $)$, où $\left(M, \phi_{M}\right)$ est un chtouca local minuscule sur $B / I$, et Fil est un sous-B-module facteur direct du B-module localement libre $\alpha^{*} M /(z-\pi) \alpha^{*} M$, qui relève le sous-B/I-module facteur direct $\psi_{M}(M) /(z-\pi)^{\tau} M d u B / I$-module localement libre ${ }^{\tau} M /(z-\pi)^{\tau} M=\left(\alpha^{*} M /(z-\pi) \alpha^{*} M\right) \otimes_{B} B / I$.

Dans le lemme on a noté $\quad \alpha^{*} M=M \otimes_{\widehat{\vartheta} \widehat{\otimes}(B / I), 1 \widehat{\otimes} \alpha} \theta \widehat{\otimes} B$. On a

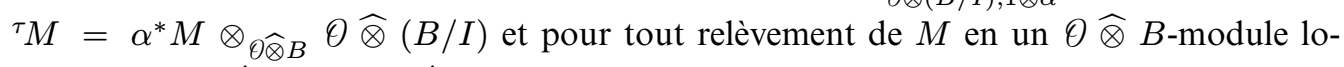
calement libre $\hat{M}, \alpha^{*} M={ }^{\tau} \hat{M}$. Ce lemme est la proposition 3.1.2 de [26]. Nous en rappelons la démonstration pour la commodité du lecteur. 
Démonstration du lemme 6.4. - D'abord il revient au même de se donner un sous- $B$-module Fil de $\alpha^{*} M /(z-\pi) \alpha^{*} M$, ou un $\oslash \widehat{\otimes} B$-module $\hat{M}$ muni d'une inclusion $\psi_{\hat{M}}$ dans $\alpha^{*} M$, et contenant $(z-\pi) \alpha^{*} M$ (et on notera $\phi_{\hat{M}}: \alpha^{*} M \rightarrow \hat{M}$ le composé de la multiplication $\operatorname{par}(z-\pi)$ et de l'inclusion $\left.(z-\pi) \alpha^{*} M \subset \hat{M}\right)$. De plus Fil est facteur direct si et seulement si $\hat{M}$ est un $\theta \widehat{\otimes} B$-module libre localement pour la topologie de Zariski sur Spec $B$. En effet si $\hat{M}$ est un $\theta \widehat{\otimes} B$-module libre localement pour la topologie de Zariski $\operatorname{sur} \operatorname{Spec} B$, il résulte du a) du lemme 1.1 que Fil $=\hat{M} /(z-\pi) \alpha^{*} M$ est facteur direct du $B$-module localement libre $\alpha^{*} M /(z-\pi) \alpha^{*} M$. Réciproquement si Fil est facteur direct, alors localement pour la topologie de Zariski sur Spec $B$ on peut trouver une base $\left(e_{1}, \ldots, e_{r}\right)$ du $B$-module $\alpha^{*} M /(z-\pi) \alpha^{*} M$ et $k \in\{0, \ldots, r\}$ tels que $e_{1}, \ldots, e_{k}$ engendrent Fil, puis relever cette base en une base $f_{1}, \ldots, f_{r}$ de $\alpha^{*} M$ comme $\theta \widehat{\otimes} B$-module et alors

$$
\left(f_{1}, \ldots, f_{k},(z-\pi) f_{k+1}, . .,(z-\pi) f_{r}\right)
$$

est une base de $\hat{M}$ comme $\theta \widehat{\otimes} B$-module. Enfin $\hat{M}$ relève $M$ si et seulement si le sous$B$-module facteur direct Fil de $\alpha^{*} M /(z-\pi) \alpha^{*} M$ relève le sous- $B / I$-module localement libre

$$
\psi_{M}(M) /(z-\pi)^{\tau} M \subset{ }^{\tau} M /(z-\pi)^{\tau} M=\left(\alpha^{*} M /(z-\pi) \alpha^{*} M\right) \otimes_{B} B / I .
$$

Suite de la démonstration de la proposition 6.3. - Le lemme 6.4 implique par récurrence la proposition (et en constitue aussi un cas particulier) car si $\gamma(I)=0$, on a un isomorphisme canonique $\mathbb{D}\left(M, \phi_{M}\right)_{B}=\alpha^{*} M /(z-\pi) \alpha^{*} M$. En effet c'est une assertion locale sur $\operatorname{Spec}(B / I)$ donc il suffit de l'établir pour $\left(M, \phi_{M}\right)$ relevable. Si $f:\left(M_{1}, \phi_{M_{1}}\right) \rightarrow\left(M_{2}, \phi_{M_{2}}\right)$ est un morphisme de chtoucas locaux minuscules sur $B / I$ relevables et si $\left(\widetilde{M}_{1}, \phi_{\widetilde{M}_{1}}\right)$ et $\left(\widetilde{M}_{2}, \phi_{\widetilde{M}_{2}}\right)$ sont des chtoucas locaux minuscules sur $B$ relevant $\left(M_{1}, \phi_{M_{1}}\right)$ et $\left(M_{2}, \phi_{M_{2}}\right)$ alors le diagramme suivant est commutatif

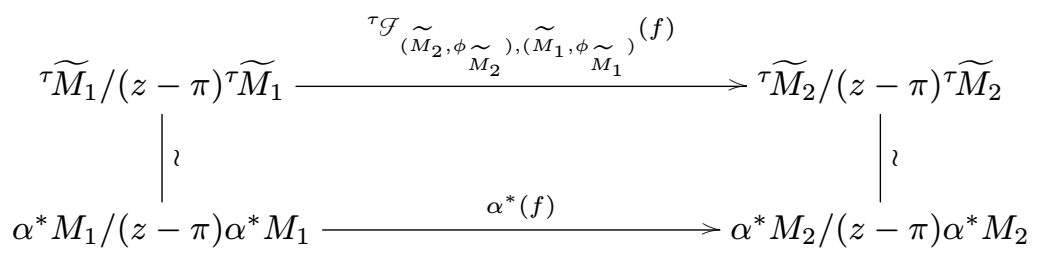

car si $\tilde{f}$ relève $f$, le morphisme

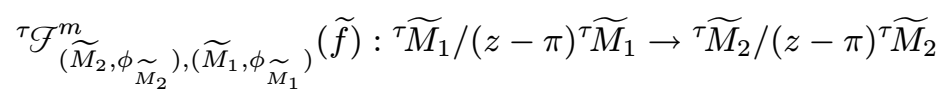

est nul pour $m>0$ et vaut $\tau \tilde{f}=\alpha^{*}(f)$ pour $m=0$.

\section{Structures de Hodge-Pink des isochtoucas locaux sur un anneau de valuation discrète}

À partir de maintenant nous supposons que la base est le spectre d'un anneau de valuation discrète complet, sauf dans le contre-exemple du paragraphe 9 où la valuation cessera d'être discrète. Dans ce paragraphe nous reprenons les constructions des paragraphes 2 et 3 (dont certaines démonstrations se simplifient) et nous énonçons le théorème « faiblement admissible implique admissible » dont la démonstration occupe le paragraphe suivant. 
Soit $\theta_{L}$ un anneau de valuation discrète complet de corps résiduel $k$. On note $m_{L}$ son idéal maximal. Soit $\pi_{L}$ une uniformisante de $\theta_{L}$. Le choix de $\pi_{L}$ détermine un isomorphisme $\theta_{L}=k\left[\left[\pi_{L}\right]\right]$. On supposera que $k$ est parfait. Cette hypothèse servira seulement dans la démonstration de la proposition 8.5. La méthode de Hartl (voir [31]) montre que le théorème «faiblement admissible implique admissible » est vrai sans cette hypothèse.

On note $L$ le corps des fractions de $\theta_{L}$. On suppose $\theta_{L}$ muni d'une structure de $\theta$-algèbre de sorte que l'image de $\pi$ dans $\theta_{L}$ soit un élément non nul de $m_{L}$. On a $\vartheta \widehat{\otimes} \theta_{L}=\theta_{L}[[z]]=k\left[\left[z, \pi_{L}\right]\right]$.

Nous allons classifier les isochtoucas locaux sur $\theta_{L}$ (c'est-à-dire les classes d'isogénie des chtoucas locaux sur $\theta_{L}$ ) à l'aide des isochtoucas locaux sur $k$ munis de structures de HodgePink définies sur $L$. On rappelle, d'après la proposition 2.2 et la définition 2.1, que pour $s, t \in \mathbb{Z}$, un isochtouca local sur $\vartheta_{L}$ d'amplitude $\subset[s, t]$ est un $\vartheta \widehat{\otimes} \vartheta_{L}\left[\frac{1}{z}\right]$-module libre $N$ muni d'un isomorphisme de $\theta \widehat{\otimes} \vartheta_{L}\left[\frac{1}{z}, \frac{1}{z-\pi}\right]$-modules $\phi_{N}:{ }^{\tau} N\left[\frac{1}{z-\pi}\right] \rightarrow N\left[\frac{1}{z-\pi}\right]$ qui vérifie la condition

(PIL) : pour un (ou pour tout) $\Theta \widehat{\otimes} \theta_{L}$-module libre $M$ muni d'un isomorphisme $M\left[\frac{1}{z}\right]=N$, il existe une constante $C$, telle que pour tout $n \in \mathbb{N}^{*}$,

$$
\begin{aligned}
& \phi_{N}{ }^{\tau} \phi_{N} \ldots \tau^{n-1} \phi_{N} \in z^{-C}(z-\pi)^{s} \cdots\left(z-\pi^{q^{n-1}}\right)^{s} \operatorname{Hom}_{\vartheta \widehat{\vartheta} \vartheta_{L}}\left({ }^{\tau^{n}} M, M\right) \\
& \text { et } \quad\left(\phi_{N}{ }^{\tau} \phi_{N} \ldots{ }^{\tau^{n-1}} \phi_{N}\right)^{-1} \in z^{-C}(z-\pi)^{-t} \cdots\left(z-\pi^{q^{n-1}}\right)^{-t} \operatorname{Hom}_{\vartheta \widehat{\otimes} \vartheta_{L}}\left(M,{ }^{n} M\right) .
\end{aligned}
$$

Pour tout isochtouca local $\left(N, \phi_{N}\right)$ sur $\theta_{L}$, si on note $\left(D, \phi_{D}\right)$ sa réduction modulo $\pi_{L}$ (qui est un isochtouca local sur $k$, c'est-à-dire que $D$ est un $k((z))$-espace vectoriel muni d'un isomorphisme de $k((z))$-espaces vectoriels $\phi_{D}:{ }^{\tau} D \rightarrow D$ ) il existe un unique isomorphisme $\rho$, congru à Id modulo $\pi_{L}$ entre les isochtoucas locaux

$$
\left(N \otimes_{\vartheta \widehat{\otimes} \vartheta_{L}\left[\frac{1}{z}\right]} \oslash \widehat{\otimes}\left(\theta_{L} / \pi \theta_{L}\right)\left[\frac{1}{z}\right], \phi_{N} \otimes 1\right) \text { et }\left(D \otimes_{k((z))} \theta \widehat{\otimes}\left(\theta_{L} / \pi \theta_{L}\right)\left[\frac{1}{z}\right], \phi_{D} \otimes 1\right)
$$

$\operatorname{sur} \theta_{L} / \pi \theta_{L}$ En effet $\phi_{N} \otimes 1$ et $\phi_{D} \otimes 1$ sont inversibles et si $k \in \mathbb{N}$ est tel que $\pi_{L}^{q^{k}} \in \pi \theta_{L}$ et si $\widetilde{\rho}: N \otimes_{\partial \widehat{\otimes} \vartheta_{L}\left[\frac{1}{z}\right]} \oslash \widehat{\otimes}\left(\vartheta_{L} / \pi \theta_{L}\right)\left[\frac{1}{z}\right] \rightarrow D \otimes_{k((z))} \oslash \widehat{\otimes}\left(\theta_{L} / \pi \theta_{L}\right)\left[\frac{1}{z}\right]$ est un morphisme arbitraire de $\theta \widehat{\otimes}\left(\theta_{L} / \pi \theta_{L}\right)\left[\frac{1}{z}\right]$-modules congru à Id modulo $\pi_{L}$, on a $\rho=\left(\phi_{D} \otimes 1\right) \ldots \tau^{k-1}\left(\phi_{D} \otimes 1\right)^{\tau^{k}}(\widetilde{\rho})^{\tau^{k-1}}\left(\phi_{N} \otimes 1\right)^{-1} \cdots\left(\phi_{N} \otimes 1\right)^{-1}$. L'existence d'un isomorphisme $\rho$ congru à Id modulo $m_{L}$ ne serait plus vraie en général si $\theta_{L}$ n'était pas de valuation discrète.

C'est pourquoi nous pouvons adopter dans ce paragraphe un point de vue plus naif que dans les paragraphes 2 et 3 , puisque la rigidification modulo $\pi$ est automatique et ne fait plus partie de la donnée. La définition suivante a pour but de simplifier la terminologie : on dira souvent isochtouca au lieu de « isochtouca local sur $k$ » et isochtouca de Hodge-Pink au lieu de « isochtouca local sur $k$ muni d'une structure de Hodge-Pink définie sur $L »$.

Le morphisme $\operatorname{Id}_{\vartheta} \widehat{\otimes} \operatorname{Fr}: \Theta \widehat{\otimes} k \rightarrow \emptyset \widehat{\otimes} k$ s'étend naturellement à $k((z))$ : il envoie $z$ sur $z$ et agit par $x \mapsto x^{q}$ sur $k$. Pour tout $k((z))$-espace vectoriel $D$ on note

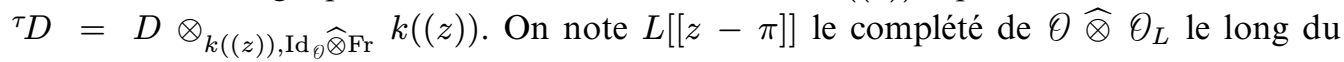
point générique du graphe du morphisme de $\theta$ vers $\theta_{L}$. Il est muni naturellement d'une structure de $k((z))$-algèbre (l'image de $z$ est $\pi+(z-\pi)$ ). 
Définition 7.1. - Un isochtouca $\left(D, \phi_{D}\right)$ est un $k((z))$-espace vectoriel $D$ muni d'un isomorphisme de $k((z))$-espaces vectoriels $\phi_{D}:{ }^{\tau} D \rightarrow D$. On note $U_{D}={ }^{\tau} D \otimes_{k((z))} L[[z-\pi]]$ le réseau tautologique de ${ }^{\tau} D \otimes_{k((z))} L((z-\pi))$.

Une structure de Hodge-Pink est la donnée d'un $L[[z-\pi]]$-réseau $V$ dans $U_{D} \otimes_{L[[z-\pi]]} L((z-\pi))={ }^{\tau} D \otimes_{k((z))} L((z-\pi))$. On dit que $V$ est d'amplitude $\subset[s, t]$ si $(z-\pi)^{-s} U_{D} \subset V \subset(z-\pi)^{-t} U_{D}$.

On appelle isochtouca de Hodge-Pink $\left(D, \phi_{D}, V_{D}\right)$ un isochtouca $\left(D, \phi_{D}\right)$ muni d'une structure de Hodge-Pink $V_{D}$.

Un morphisme entre deux isochtoucas $\left(D, \phi_{D}\right)$ et $\left(D^{\prime}, \phi_{D^{\prime}}\right)$ est un morphisme de $k((z))$-espaces vectoriels $f: D \rightarrow D^{\prime}$ tel que $f \circ \phi_{D}=\phi_{D^{\prime}} \circ^{\tau} f$.

Un morphisme entre deux isochtoucas de Hodge-Pink $\left(D, \phi_{D}, V_{D}\right)$ et $\left(D^{\prime}, \phi_{D^{\prime}}, V_{D^{\prime}}\right)$ est un morphisme d'isochtoucas $f$ de $\left(D, \phi_{D}\right)$ vers $\left(D^{\prime}, \phi_{D^{\prime}}\right)$ tel que $f\left(V_{D}\right) \subset V_{D^{\prime}}$.

On notera parfois $D$ au lieu de $\left(D, \phi_{D}\right)$ ou de $\left(D, \phi_{D}, V_{D}\right)$.

Si $D$ est de dimension 1, la matrice de $\Phi_{D}$ dans une base est le produit de $z^{k}$ et d'une unité. De plus on a $V_{D}=(z-\pi)^{l} U_{D}$. On note $t_{N}(D)=k$ et $t_{H}(D)=-l$. On notera que l'amplitude de $V_{D}$ est $\left[t_{H}(D), t_{H}(D)\right]$. On devrait noter $t_{N}\left(D, \phi_{D}\right)$ et $t_{H}\left(D, \phi_{D}, V_{D}\right)$ mais c'est trop lourd.

Si $D$ est de dimension $r$, on note $\operatorname{det}(D)=\Lambda^{r} D, \phi_{\operatorname{det}(D)}=\operatorname{det}\left(\phi_{D}\right) \quad$ et $V_{\operatorname{det}(D)}=\operatorname{det}\left(V_{D}\right)$. Alors $\operatorname{det}(D)$ est un isochtouca de Hodge-Pink de dimension 1. On pose alors $t_{N}(D)=t_{N}(\operatorname{det}(D))$ et $t_{H}(D)=t_{H}(\operatorname{det}(D))$.

$\mathrm{Si}\left(D, \phi_{D}\right)$ est un isochtouca on appelle sous-isochtouca un sous- $k((z))$-espace vectoriel $D^{\prime}$ tel que $\phi_{D}\left({ }^{\tau} D^{\prime}\right)=D^{\prime}$, et on prend évidemment pour $\phi_{D^{\prime}}$ la restriction de $\phi_{D}$ à ${ }^{\tau} D^{\prime}$. Si $D$ est un isochtouca de Hodge-Pink on munit $D^{\prime}$ d'une structure de Hodge-Pink en posant $V_{D^{\prime}}=V_{D} \cap\left({ }^{\tau} D^{\prime} \otimes_{k((z))} L((z-\pi))\right)$.

Définition 7.2. - Un isochtouca de Hodge-Pink $D$ est dit faiblement admissible si $t_{H}(D)=t_{N}(D)$ et pour tout sous-isochtouca $D^{\prime}$ on a $E x t t_{H}\left(D^{\prime}\right) \leq t_{N}\left(D^{\prime}\right)$.

Remarque. - Soit $D$ faiblement admissible. Pour tout isochtouca de Hodge-Pink $\left(D^{\prime}, \phi_{D^{\prime}}, V_{D^{\prime}}\right)$ et tout morphisme injectif $f: D^{\prime} \rightarrow D$ d'isochtoucas de Hodge-Pink, on a $V_{D^{\prime}} \subset V_{D} \cap\left({ }^{\tau} D^{\prime} \otimes_{k((z))} L((z-\pi))\right)$ et donc $t_{H}\left(D^{\prime}\right) \leq t_{N}\left(D^{\prime}\right)$.

Voici notre résultat principal. Dans [31], Hartl a obtenu ce résultat (sur une base un peu plus générale) par une méthode complètement différente. Nous allons construire un foncteur $\mathbb{D}_{\text {iso }}^{+}$de la catégorie des isochtoucas locaux sur $\theta_{L}$ vers la catégorie des isochtoucas de HodgePink faiblement admissibles, qui vérifiera le théorème suivant.

THÉORÈME 7.3. - Le foncteur $\mathbb{D}_{\text {iso }}^{+}$est une équivalence de catégories.

Le qualificatif iso indique que $\mathbb{D}_{\text {iso }}^{+}$relie des catégories à isogénies près et + signale la donnée supplémentaire de la structure de Hodge-Pink.

La démonstration du théorème 7.3 occupe la fin de ce paragraphe ainsi que le paragraphe suivant. Nous commençons par construire un foncteur (encore noté $\mathbb{D}_{\text {iso }}^{+}$par abus) de la catégorie des isochtoucas locaux vers celle des isochtoucas de Hodge-Pink (en reprenant la définition 3.11) et nous montrons que son image est contenue dans la sous-catégorie pleine des isochtoucas de Hodge-Pink faiblement admissibles et qu'il est pleinement fidèle (cela est 
un cas particulier de la proposition 3.14). Dans le paragraphe suivant nous montrerons que l'image essentielle est formée de tous les isochtoucas de Hodge-Pink faiblement admissibles, c'est-à-dire que « faiblement admissible implique admissible ».

On notera $\mathscr{C}$ l'anneau formé des séries à coefficients dans $\theta_{L}$ en la variable $z$ et son inverse qui convergent absolument sur le disque rigide épointé $\{0<|z|<1\}$. C'est l'anneau noté $\mathscr{C}\left(\theta_{L}\right)$ au paragraphe 3 . On note $v$ la valuation sur $\theta_{L}$ (toujours normalisée pour que $v(\pi)=1$ ). Les définitions du début du paragraphe 3 , appliquées à $B=\theta_{L}$, donnent $\nu: \theta_{L} \rightarrow \mathbb{N} \cup\{+\infty\}$ et $\nu^{\prime}: \theta_{L} \rightarrow \mathbb{R}_{+} \cup\{+\infty\}$. Il est clair que $\nu^{\prime}=v$ et que $\nu$ est la partie entière de $v$. Tous les résultats démontrés dans le paragraphe 3 sont vrais ici avec $v$ à la place de $\nu$, et nous allons les rappeler maintenant, car il est plus commode pour la suite d'utiliser $v$ plutôt que $\nu$.

On a

$$
\mathscr{C}=\left\{\sum_{n \in \mathbb{Z}} a_{n} z^{n}, a_{n} \in \mathscr{\theta}_{L}, \lim _{n \mapsto-\infty} \frac{v\left(a_{n}\right)}{|n|}=+\infty\right\}=\bigcap_{m \in \mathbb{N}^{*}}\left(k\left[\left[z, \pi_{L}\right]\right]\left[\left[\frac{\pi^{q^{m}}}{z}\right]\right]\left[\frac{1}{z}\right]\right) .
$$

Dans $\mathscr{C}$ on a l'élément particulier

$$
\alpha=\left(1-\frac{\pi}{z}\right)\left(1-\frac{\pi^{q}}{z}\right)\left(1-\frac{\pi^{q^{2}}}{z}\right) \cdots
$$

Soient $s, t \in \mathbb{Z}$ et $\left(N, \phi_{N}\right)$ un isochtouca local sur $\theta_{L}$ d'amplitude $\subset[s, t]$. On veut lui associer un isochtouca de Hodge-Pink $D$, c'est-à-dire un $k((z))$-espace vectoriel $D$ muni d'un isomorphisme de $k((z))$-espaces vectoriels $\phi_{D}:{ }^{\tau} D \rightarrow D$, et d'un $L[[z-\pi]]$-réseau $V_{D}$ dans ${ }^{\tau} D \otimes_{k((z))} L((z-\pi))$.

On remarque d'abord que

$$
\oslash \widehat{\otimes} \theta_{L}\left[\frac{1}{z}\right] /\left(\pi_{L}\right)=\theta \widehat{\otimes} \theta_{L}\left[\frac{1}{z}, \frac{1}{z-\pi}\right] /\left(\pi_{L}\right)=k((z)) .
$$

On commence par poser

$$
D=N \otimes_{\partial \widehat{\otimes} \vartheta_{L}\left[\frac{1}{z}\right]} k((z))=N / \pi_{L} N \text { et } \phi_{D}=\phi_{N} \bmod \pi_{L} .
$$

Le lemme suivant résulte du lemme 3.10 et du fait qu'une rigidification modulo $\pi$ est équivalente à une rigidification modulo $\pi_{L}$, comme nous l'avons expliqué au début de ce paragraphe. Nous reprenons la démonstration sous une forme matricielle qui nous servira ensuite.

Lemme 7.4. - Il existe un unique morphisme $R$ de $\mathscr{C}\left[\frac{1}{\alpha}\right]$-modules congru à Id modulo $\pi_{L}$ de $N \otimes_{\oslash \widehat{\otimes}_{L}\left[\frac{1}{z}\right]} \mathscr{C}\left[\frac{1}{\alpha}\right]$ vers $D \otimes_{k((z))} \mathscr{C}\left[\frac{1}{\alpha}\right]$ tel que l'on ait

$$
R\left(\phi_{N} \otimes 1\right)=\left(\phi_{D} \otimes 1\right)^{\tau} R .
$$

De plus c'est un isomorphisme de $\mathscr{C}\left[\frac{1}{\alpha}\right]$-modules.

Démonstration. - Nous fixons une base de $N$ sur $\oslash \widehat{\otimes} \vartheta_{L}\left[\frac{1}{z}\right]$. Par réduction modulo $\pi_{L}$ on en déduit une base de $D$ sur $k((z))$. On note encore $\phi_{N}$ et $\phi_{D}$ les matrices de $\phi_{N}$ et $\phi_{D}$ dans ces bases. On a $\phi_{N} \in G L_{r}\left(\theta \widehat{\otimes} \vartheta_{L}\left[\frac{1}{z}, \frac{1}{z-\pi}\right]\right)$ et $\phi_{D}=\phi_{N} \bmod \pi_{L}$. Supposons d'abord $\phi_{N}^{-1} \in M_{r}\left(\Theta \widehat{\otimes} \vartheta_{L}\left[\frac{1}{z}\right]\right)$, c'est-à-dire que l'isochtouca local $\left(N, \phi_{N}\right)$ est d'amplitude $\subset[s, 0]$ pour un certain $s \in \mathbb{Z}$. On pose alors $R=\lim _{n \mapsto+\infty} r_{n} \in M_{r}(\mathscr{C})$ où

$$
r_{n}=\phi_{D}{ }^{\tau} \phi_{D} \tau^{2} \phi_{D} \ldots \tau^{n-1} \phi_{D} \tau^{n-1} \phi_{N}^{-1} \ldots \tau^{2} \phi_{N}^{-1 \tau} \phi_{N}^{-1} \phi_{N}^{-1} \in M_{r}\left(\theta \widehat{\otimes} \theta_{L}\left[\frac{1}{z}\right]\right)
$$


Soit $C$ une constante telle que $\phi_{D} \in z^{-C} M_{r}(k[[z]])$ et $\phi_{N}^{-1} \in z^{-C} M_{r}\left(\theta \widehat{\otimes} \theta_{L}\right)$. Alors $r_{n} \in z^{-2 C n} M_{r}\left(\theta \widehat{\otimes} \theta_{L}\right)$ et $r_{n+1}-r_{n} \in \pi_{L}^{q^{n}} z^{-2 C(n+1)} M_{r}\left(\theta \widehat{\otimes} \theta_{L}\right)$, ce qui montre que la limite des $r_{n}$, au sens de la topologie $\pi$-adique, appartient bien à $M_{r}(\mathscr{C})$. En fait $\mathscr{C}=\mathscr{C}\left(\vartheta_{L}\right)$ est complet pour la topologie introduite avant le lemme 3.1 et la suite $r_{n}$ est de Cauchy et converge pour cette topologie. Par ailleurs il est évident que $R$ est congru à Id modulo $\pi_{L}$ et que $R \phi_{N}=\phi_{D}^{\tau} R$. Si $r=1$ et $\phi_{N}=(z-\pi)^{-1}$, on obtient $R=\left(1-\frac{\pi}{z}\right)\left(1-\frac{\pi^{q}}{z}\right) \cdots$, c'est-à-dire que $R$ est égal à $\alpha$.

Si on enlève maintenant l'hypothèse que $\phi_{N}^{-1}$ appartient à $M_{r}\left(\theta \widehat{\otimes} \theta_{L}\left[\frac{1}{z}\right]\right)\left(\right.$ donc si $\phi_{N}$ est d'amplitude $\subset[s, t]$ arbitraire $), \phi_{N}^{-1}(z-\pi)^{t}$ appartient à $E x t M_{r}\left(\theta \widehat{\otimes} \theta_{L}\left[\frac{1}{z}\right]\right)$. Si on pose $N^{\prime}=N=\left(\theta \widehat{\otimes} \theta_{L}\left[\frac{1}{z}\right]\right)^{r}$ et $\phi_{N^{\prime}}=\phi_{N}(z-\pi)^{-t}$ (si bien que $\left(N^{\prime}, \phi_{N^{\prime}}\right)$ est un isochtouca local d'amplitude $\subset[s-t, 0])$, et $R^{\prime}$ la matrice associée à $\phi_{N^{\prime}}$ par la construction ci-dessus, on pose $R=R^{\prime} \alpha^{-t} \in M_{r}\left(\mathscr{C}\left[\frac{1}{\alpha}\right]\right)$.

On peut définir $R^{-1}$ de la même façon (en commençant par le cas où $\phi_{N} \in M_{r}\left(\theta \widehat{\otimes} \theta_{L}\left[\frac{1}{z}\right]\right)$, i.e. $\left(N, \phi_{N}\right)$ d'amplitude $\subset[0, t]$ pour $\left.t \in \mathbb{N}\right)$ et montrer que $R^{-1}$ appartient aussi à $M_{r}\left(\mathscr{C}\left[\frac{1}{\alpha}\right]\right)$. Il en résulte que $R \in G L_{r}\left(\mathscr{C}\left[\frac{1}{\alpha}\right]\right)$.

Ceci termine la démonstration de l'existence de $R$, qui est un isomorphisme de $\mathscr{C}\left[\frac{1}{\alpha}\right]$-modules. Pour l'unicité, on remarque que si $R^{\sharp}$ est un morphisme de $\mathscr{C}\left[\frac{1}{\alpha}\right]$-modules de $N \otimes_{\partial \widehat{\otimes} \vartheta_{L}\left[\frac{1}{z}\right]} \mathscr{C}\left[\frac{1}{\alpha}\right]$ vers $D \otimes_{k((z))} \mathscr{C}\left[\frac{1}{\alpha}\right]$, congru à Id modulo $\pi_{L}$, tel que l'on ait $R^{\sharp}\left(\phi_{N} \otimes 1\right)=$ $\left(\phi_{D} \otimes 1\right)^{\tau} R^{\sharp}$, alors $R^{\sharp} R^{-1}$ est un endomorphisme de $\mathscr{C}\left[\frac{1}{\alpha}\right]$-modules de $D \otimes_{k((z))} \mathscr{C}\left[\frac{1}{\alpha}\right]$, congru à Id modulo $\pi_{L}$, tel que $R^{\sharp} R^{-1}\left(\phi_{D} \otimes 1\right)=\left(\phi_{D} \otimes 1\right)^{\tau}\left(R^{\sharp} R^{-1}\right)$. Comme $R^{\sharp} R^{-1}$ est congru à Id modulo $\pi_{L},{ }^{\tau}\left(R^{\sharp} R^{-1}\right)$ est congru à Id modulo $\pi_{L}^{q}$, donc $R^{\sharp} R^{-1}$ est congru à Id modulo $\pi_{L}^{q}$, donc ${ }^{\tau}\left(R^{\sharp} R^{-1}\right)$ est congru à Id modulo $\pi_{L}^{q^{2}}$, donc $R^{\sharp} R^{-1}$ est congru à Id modulo $\pi_{L}^{q^{2}}$, etc. Ceci termine la démonstration de l'unicité.

Nous allons maintenant définir la structure de Hodge-Pink sur $D$, en reprenant la définition 3.11. Il y a un morphisme évident $c_{\pi}: \mathscr{C} \rightarrow L[[z-\pi]]$, et il s'étend en des morphismes $\mathscr{C}\left[\frac{1}{\tau_{\alpha}}\right] \rightarrow L[[z-\pi]]$ et $\mathscr{C}\left[\frac{1}{\alpha}\right] \rightarrow L((z-\pi))$. Par conséquent $R$ s'étend en un isomor-

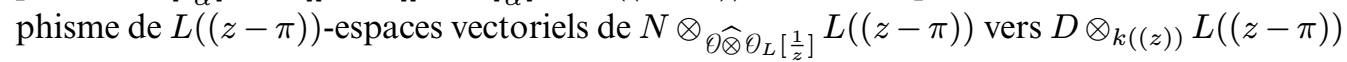

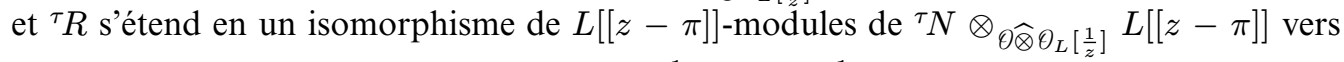
${ }^{\tau} D \otimes_{k((z))} L[[z-\pi]]$. D'autre part $\phi_{N}:{ }^{\tau} N\left[\frac{1}{z-\pi}\right] \rightarrow N\left[\frac{1}{z-\pi}\right]$ s'étend en un isomorphisme de

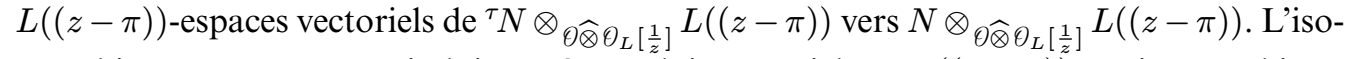
morphisme $\phi_{D}:{ }^{\tau} D \rightarrow D$ induit, après produit tensoriel par $L((z-\pi))$, un isomorphisme $\phi_{D} \otimes 1:{ }^{\tau} D \otimes_{k((z))} L((z-\pi)) \rightarrow D \otimes_{k((z))} L((z-\pi))$.

On termine la construction du foncteur $\mathbb{D}_{\text {iso }}^{+}$en définissant la structure de Hodge-Pink $V_{D}$ comme le $L[[z-\pi]]$ réseau de ${ }^{\tau} D \otimes_{k((z))} L((z-\pi))$ égal à

$$
\begin{aligned}
V_{D} & =\left(\phi_{D} \otimes 1\right)^{-1}\left(R\left(N \otimes_{\vartheta_{\widehat{\otimes} \vartheta_{L}\left[\frac{1}{z}\right]}} L[[z-\pi]]\right)\right) \\
& ={ }^{\tau} R\left(\left(\phi_{N} \otimes 1\right)^{-1}\left(N \otimes_{\vartheta_{\widehat{\otimes} \vartheta_{L}\left[\frac{1}{z}\right]}} L[[z-\pi]]\right)\right) .
\end{aligned}
$$

C'est la structure de Hodge-Pink associée à $\left(N, \phi_{N}\right)$ au sens de la définition 3.11.

Proposition 7.5. - L'isochtouca de Hodge-Pink $\left(D, \phi_{D}, V_{D}\right)$ associé à un isochtouca local $\left(N, \phi_{N}\right)$ par la construction ci-dessus est faiblement admissible. 
Démonstration. - Nous allons introduire une famille de valuations sur $\mathscr{C}$ et les étendre à $\mathscr{C}\left[\frac{1}{\alpha}\right]$. Soit $w \in\left[0,+\infty\left[\right.\right.$. Pour tout élément $x \in \mathscr{C}$ on note $v_{w}(x) \in[0,+\infty]$ "le minimum des valuations des valeurs de $x$ lorsque $z$ est de valuation $w$ dans un anneau de valuation réelle contenant $\theta_{L}$, dont la valuation est normalisée par valuation $(\pi)=1$ ". Plus précisément pour $x=\sum_{n \in \mathbb{Z}} x_{n} z^{n} \in \mathscr{C}$ on pose $v_{w}(x)=\inf _{n \in \mathbb{Z}} n w+v\left(x_{n}\right)$. Si on écrit $\Theta \widehat{\otimes} \theta_{L}=k\left[\left[z, \pi_{L}\right]\right]$, et si $x=\sum_{a \in \mathbb{Z}, b \in \mathbb{N}} x_{a, b} z^{a} \pi_{L}^{b}$, avec $x_{a, b} \in k$, on a $v_{w}(x)=\inf _{(a, b)}$ tels que $x_{a, b} \neq 0$ aw $+\frac{b}{e}$, où $e$ est l'indice de ramification de $L$ sur $K$ (c'est-àdire l'unique entier tel que $\pi / \pi_{L}^{e}$ est une unité de $\left.\theta_{L}\right)$. Par exemple $v_{w}(z-\pi)=\min (w, 1)$.

Lemme 7.6. - Pour tout $w \in\left[0,+\infty\left[, v_{w}\right.\right.$ est une valuation sur $\mathscr{C}$ et s'étend de manière unique en une valuation sur $\mathscr{C}\left[\frac{1}{\alpha}\right]$.

On a $v_{w}\left(1-\frac{\pi}{z}\right)=\min (0,1-w), v_{w}\left(1-\frac{\pi^{q}}{z}\right)=\min (0, q-w)$, et $v_{w}(\alpha)=$ $\sum_{i=0}^{\infty} \min \left(0, q^{i}-w\right)$. On remarque que si $\nu_{w}$ est défini comme dans le paragraphe 3 on a $\nu_{w} \leq v_{w} \leq \nu_{w}+1$. Un avantage de $v_{w}$ sur $\nu_{w}$ est que le lemme 7.6 est immédiat alors que $\nu_{w}$ ne vérifie que des énoncés plus faibles (comme par exemple le lemme 3.5 qui avait demandé une démonstration).

Le lemme suivant énumère les propriétés de $R$ que nous utiliserons pour montrer la proposition 7.5 .

\section{Lemme 7.7. - On a}

a) R est un isomorphisme de $\mathscr{C}\left[\frac{1}{\alpha}\right]$-modules de $N \otimes_{\oslash \widehat{\otimes} \vartheta_{L}\left[\frac{1}{z}\right]} \mathscr{C}\left[\frac{1}{\alpha}\right]$ vers $D \otimes_{k((z))} \mathscr{C}\left[\frac{1}{\alpha}\right]$,

b) $R\left(\phi_{N} \otimes 1\right)=\left(\phi_{D} \otimes 1\right)^{\tau} R$,

c) $R$ est congru à $\operatorname{Id}$ modulo $\pi_{L}$,

d) $V_{D}={ }^{\tau} R\left(\left(\phi_{N} \otimes 1\right)^{-1}\left(N \otimes_{\partial \widehat{\otimes} \vartheta_{L}\left[\frac{1}{z}\right]} L[[z-\pi]]\right)\right)$,

e) si on choisit une base de $N$ sur $\Theta \widehat{\otimes} \vartheta_{L}\left[\frac{1}{z}\right]$, et si on munit $D$ de la base qui s'en déduit par réduction modulo $\pi_{L}$, il existe une constante $C$ telle que pour tout entier $n \in \mathbb{N}$, on $a v_{q^{n}}\left(\left(\phi_{D} \phi_{D} \ldots \tau^{n-1} \phi_{D}\right)^{-1} R\right) \geq-C q^{n}$ et $v_{q^{n}}\left(R^{-1}\left(\phi_{D}{ }^{\tau} \phi_{D} \ldots \tau^{n-1} \phi_{D}\right)\right) \geq-C q^{n}$.

Dans e), on convient que la valuation d'une matrice est le minimum des valuations de ses coefficients.

Démonstration. - Voici la démonstration de e). On suppose $\phi_{N}$ d'amplitude $\subset[s, t]$. D'après la condition (PIL), il existe $C \in \mathbb{N}$ tel que pour tout $n \in \mathbb{N}$,

$$
\begin{aligned}
\phi_{N}{ }^{\tau} \phi_{N} \cdots \tau^{n-1} \phi_{N} & \in z^{-C}(z-\pi)^{s} \cdots\left(z-\pi^{q^{n-1}}\right)^{s} M_{r}\left(\Theta \widehat{\otimes} \vartheta_{L}\right) \\
\text { et } \quad\left(\phi_{N}{ }^{\tau} \phi_{N} \cdots \tau^{n-1} \phi_{N}\right)^{-1} & \in z^{-C}(z-\pi)^{-t} \cdots\left(z-\pi^{q^{n-1}}\right)^{-t} M_{r}\left(\oslash \widehat{\otimes} \oslash_{L}\right) .
\end{aligned}
$$

Or $v_{q^{n}}(z)=q^{n}, v_{q^{n}}(z-\pi)=1, \ldots, v_{q^{n}}\left(z-\pi^{q^{n-1}}\right)=q^{n-1}$. On a donc pour tout $n \in \mathbb{N}$, $v_{q^{n}}\left(\phi_{N}{ }^{\tau} \phi_{N} \cdots \tau^{n-1} \phi_{N}\right) \geq v_{q^{n}}\left(z^{-C}(z-\pi)^{s} \cdots\left(z-\pi^{q^{n-1}}\right)^{s}\right)=-C q^{n}+s\left(q^{n-1}+\cdots+1\right)$

et

$$
\begin{aligned}
v_{q^{n}}\left(\left(\phi_{N}^{\tau} \phi_{N} \ldots \tau^{n-1} \phi_{N}\right)^{-1}\right) & \geq v_{q^{n}}\left(z^{-C}(z-\pi)^{-t} \cdots\left(z-\pi^{q^{n-1}}\right)^{-t}\right) \\
& =-C q^{n}-t\left(q^{n-1}+\cdots+1\right) .
\end{aligned}
$$


Or

$$
\begin{aligned}
& \left(\phi_{D}{ }^{\tau} \phi_{D} \ldots \tau^{n-1} \phi_{D}\right)^{-1} R=\tau^{n} R\left(\phi_{N}{ }^{\tau} \phi_{N} \ldots \tau^{n-1} \phi_{N}\right)^{-1} \\
& R^{-1}\left(\phi_{D}{ }^{\tau} \phi_{D} \ldots \tau^{n-1} \phi_{D}\right)=\left(\phi_{N}{ }^{\tau} \phi_{N} \ldots \tau^{n-1} \phi_{N}\right)^{\tau^{n}} R^{-1}
\end{aligned}
$$

et d'autre part

$$
v_{q^{n}}\left(\tau^{n} R\right)=q^{n} v_{1}(R) \text { et } v_{q^{n}}\left(\tau^{n} R^{-1}\right)=q^{n} v_{1}\left(R^{-1}\right) .
$$

Le lemme 7.7 est démontré.

Démonstration de la proposition 7.5. - Pour tout entier $l, \Lambda^{l}(N)$ est un isochtouca local et l'isochtouca de Hodge-Pink associé est $\Lambda^{l}(D)$. En prenant $l$ égal au rang de $N$, on voit qu'il existe un entier $t \in \mathbb{Z}$ et $u \in\left(\theta \widehat{\otimes} \vartheta_{L}\left[\frac{1}{z}\right]\right)^{\times}$tels que $\phi_{\Lambda^{l}(N)}=(z-\pi)^{t} u$ dans une base de $\Lambda^{l}(N)$. On a bien sûr $V_{\Lambda^{l}(D)}=(z-\pi)^{-t} U_{\Lambda^{l}(D)}$ donc $t_{H}(D)=t$. Par la condition (PIL), il existe $C$ tel que pour tout $n \in \mathbb{N}, u^{\tau} u \ldots \tau^{n-1} u$ et son inverse appartiennent à $z^{-C} \oslash \widehat{\otimes} \vartheta_{L}$. On en déduit $u \in\left(\Theta \widehat{\otimes} \theta_{L}\right)^{\times}$et donc $t_{N}(D)=t$. Donc on a bien $t_{H}(D)=t_{N}(D)$.

Supposons par l'absurde que $D$ n'est pas faiblement admissible. Il existe alors un sousisochtouca $D^{\prime}$ de $D$ tel que $t_{H}\left(D^{\prime}\right)>t_{N}\left(D^{\prime}\right)$. En remplaçant $N$ par $\Lambda^{l}(N)$, où $l$ est la dimension de $D^{\prime}$, on se ramène à la situation où $D^{\prime}$ est de dimension 1 . Pour toute base $\left(e_{1}, \ldots, e_{r}\right)$ de $N$ sur $\Theta \widehat{\otimes} \theta_{L}\left[\frac{1}{z}\right]$, on note $\left(f_{1}, \ldots, f_{r}\right)$ la base de $D$ sur $k((z))$ obtenue par réduction modulo $\pi_{L}$. On a $k \subset \theta_{L}$ d'où $k((z)) \subset \Theta \widehat{\otimes} \Theta_{L}\left[\frac{1}{z}\right]$ et $G L_{r}(k((z))) \subset$ $G L_{r}\left(\Theta \widehat{\otimes} \vartheta_{L}\left[\frac{1}{z}\right]\right)$, donc on peut choisir la base $\left(e_{1}, \ldots, e_{r}\right)$ telle que $D^{\prime}=k((z)) f_{1}$. Notons $j=t_{N}\left(D^{\prime}\right)$ de sorte que $\phi_{D}\left(f_{1}\right)=u z^{j} f_{1}$ avec $u \in(k[[z]])^{\times}$. On suppose par l'absurde que $t_{H}\left(D^{\prime}\right)>t_{N}\left(D^{\prime}\right)$, c'est-à-dire

$$
(z-\pi)^{-(j+1) \tau} D^{\prime} \otimes_{k((z))} L[[z-\pi]] \subset\left(\phi_{D} \otimes 1\right)^{-1}\left(R\left(N \otimes_{\vartheta \widehat{\otimes} \vartheta_{L}} L[[z-\pi]]\right)\right)
$$

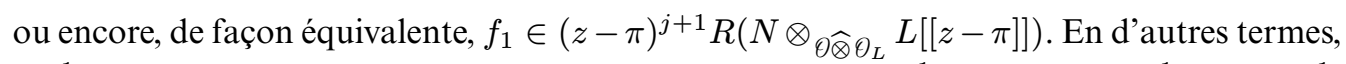
$R^{-1}\left(f_{1}\right)$ s'annule au moins à l'ordre $j+1$ en $z=\pi$. Comme $R^{-1}=\left(\phi_{N} \otimes 1\right)^{\tau} R^{-1}\left(\phi_{D} \otimes 1\right)^{-1}$, comme $\phi_{N}$ n'a pas de pôle en $z=\pi^{q}, \pi^{q^{2}}, \ldots$ et comme $D^{\prime}$ est stable par $\phi_{D}$, on voit que $R^{-1}\left(f_{1}\right)$ s'annule au moins à l'ordre $j+1$ aussi en $z=\pi^{q}, \pi^{q^{2}}, \ldots$ Le premier coefficient $R^{-1}\left(f_{1}\right)_{1} \in \mathscr{C}\left[\frac{1}{\alpha}\right]$ de $R^{-1}\left(f_{1}\right)$ dans la base $\left(e_{1}, \ldots, e_{r}\right)$ vérifie donc

- il est congru à 1 modulo $\pi_{L}$

- il s'annule au moins à l'ordre $j+1$ en $z=\pi, \pi^{q}, \pi^{q^{2}}, \ldots$

- pour tout entier $n \in \mathbb{N}^{*}$ on a $v_{q^{n}}\left(R^{-1}\left(f_{1}\right)_{1}\right) \geq-C q^{n}-j n q^{n}$ (grâce à la deuxième inégalité de la partie e) du lemme 7.7 et au fait que $\left.\phi_{D}{ }^{\tau} \phi_{D} \ldots{ }^{\tau^{n-1}} \phi_{D}{ }^{\tau^{n}} f_{1}=\left(u^{\tau} u \cdots \tau^{n-1} u\right) z^{n j} f_{1}\right)$.

Or ces propriétés sont contradictoires. D'après le lemme 3.4 (dont nous ne reprenons pas la démonstration) $\alpha^{-(j+1)} R^{-1}\left(f_{1}\right)_{1}$ appartient à $\mathscr{C}$. D'autre part cet élément est congru à 1 modulo $\pi_{L}$. Enfin, grâce au lemme 7.6 et comme $v_{q^{n}}(\alpha)=-n q^{n}+\left(q^{n-1}+\cdots+1\right)$, il existe $C$ tel que pour tout entier $n \in \mathbb{N}^{*}$ on ait

$$
v_{q^{n}}\left(\alpha^{-(j+1)} R^{-1}\left(f_{1}\right)_{1}\right) \geq-C q^{n}+n q^{n} .
$$

Or $\mathscr{C}$ ne contient aucun élément ayant de telles propriétés, car tout $x \in \mathscr{C}$ congru à 1 modulo $\pi_{L}$ vérifie $v_{w}(x) \leq 0$ pour tout $w \in \mathbb{R}_{+}$. La proposition 7.5 est démontrée. 
On a donc construit un foncteur $\mathbb{D}_{\text {iso }}^{+}$de la catégorie des isochtoucas locaux sur $\theta_{L}$ dans celle des isochtoucas de Hodge-Pink faiblement admissibles. Pour montrer le théorème 7.3 nous devons donc établir que $\mathbb{D}_{\text {iso }}^{+}$est une équivalence de catégories. La pleine fidélité de $\mathbb{D}_{\text {iso }}^{+}$ est un cas particulier de la proposition 3.14. Dans le paragraphe suivant nous montrerons que $\mathbb{D}_{\text {iso }}^{+}$est essentiellement surjectif.

Soient $\left(N, \phi_{N}\right)$ et $\left(N^{\prime}, \phi_{N^{\prime}}\right)$ des isochtoucas locaux et $\left(D, \phi_{D}, V_{D}\right)$ et $\left(D^{\prime}, \phi_{D^{\prime}}, V_{D^{\prime}}\right)$ les isochtoucas de Hodge-Pink qui leur sont associés. Notons $C_{\text {iso-cht }}$ la catégorie des isochtou-

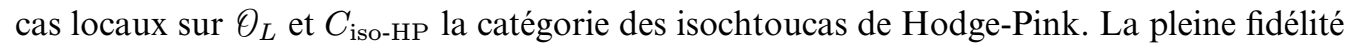
de $\mathbb{D}_{\text {iso }}^{+}$signifie que le morphisme

$$
\mathbb{D}_{\text {iso }}^{+}(.): \operatorname{Hom}_{C_{\text {iso-cht }}}\left(\left(N, \phi_{N}\right),\left(N^{\prime}, \phi_{N^{\prime}}\right)\right) \rightarrow \operatorname{Hom}_{C_{\text {iso-HP }}}\left(\left(D, \phi_{D}, V_{D}\right),\left(D^{\prime}, \phi_{D^{\prime}}, V_{D^{\prime}}\right)\right)
$$

est un isomorphisme. Par construction de $\mathbb{D}_{\text {iso }}^{+}$, ce morphisme n'est que la réduction modulo $\pi_{L}$ mais il intéressant d'avoir aussi une formule pour son inverse. On se donne $f \in \operatorname{Hom}_{C_{\text {iso-cht }}}\left(\left(N, \phi_{N}\right),\left(N^{\prime}, \phi_{N^{\prime}}\right)\right)$ et on note $h=\mathbb{D}_{\text {iso }}^{+}(f)$. On rappelle qu'il existe un unique isomorphisme $R$ de $\mathscr{C}\left[\frac{1}{\alpha}\right]$-modules congru à Id modulo $\pi_{L}$ de $N \otimes_{\partial \widehat{\otimes} \vartheta_{L}\left[\frac{1}{z}\right]} \mathscr{C}\left[\frac{1}{\alpha}\right]$ vers $D \otimes_{k((z))} \mathscr{C}\left[\frac{1}{\alpha}\right]$ tel que l'on ait $R\left(\phi_{N} \otimes 1\right)=\left(\phi_{D} \otimes 1\right)^{\tau} R$. On associe de même $R^{\prime}$ à $N^{\prime}$. Alors

$$
f=R^{\prime-1} \circ(h \otimes 1) \circ R \text { dans } \operatorname{Hom}_{\vartheta \widehat{\otimes} \vartheta_{L}\left[\frac{1}{z}\right]}\left(N, N^{\prime}\right) \otimes_{\vartheta \widehat{\otimes} \vartheta_{L}\left[\frac{1}{z}\right]} \mathscr{C}\left[\frac{1}{\alpha}\right] .
$$

En effet on a

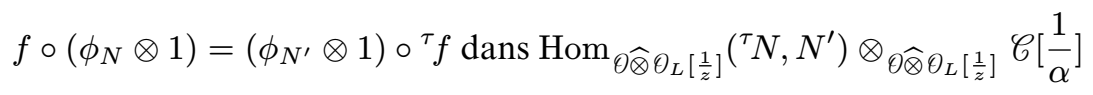

et $R^{\prime-1} \circ(h \otimes 1) \circ R$ vérifie la même équation. Comme $f$ et $R^{\prime-1} \circ(h \otimes 1) \circ R$ sont congrus modulo $\pi_{L}$, il le sont modulo $\pi_{L}^{q}, \ldots$, donc ils sont égaux.

En fait, non seulement $\mathbb{D}_{\text {iso }}^{+}$est pleinement fidèle, mais la proposition 3.12 fournit un quasi-inverse sur son image essentielle, dont on va rappeler la construction. Soient $\left(N, \phi_{N}\right)$ un isochtouca local et $\left(D, \phi_{D}, V_{D}\right)$ l'isochtouca de Hodge-Pink qui lui est associé. On pose

$$
\begin{gathered}
\widetilde{N}=\left\{x \in D \otimes_{k((z))} \mathscr{C}\left[\frac{1}{\alpha}\right], \forall n \in \mathbb{N}, x \in \phi_{D} \cdots \tau^{n} \phi_{D} \tau^{n} V_{D},\right. \\
\text { et } \left.\exists C, \forall n \in \mathbb{N}, v_{q^{n}}\left(\left(\phi_{D}{ }^{\tau} \phi_{D} \cdots \tau^{n-1} \phi_{D}\right)^{-1} x\right) \geq-C q^{n}\right\} .
\end{gathered}
$$

Bien sûr $\tau^{\tau^{n}} V_{D}$ est un $L\left[\left[z-\pi^{q^{n}}\right]\right]$-réseau de $\tau^{\tau^{n+1}} D \otimes_{k((z))} L\left(\left(z-\pi^{q^{n}}\right)\right)$ et donc $\phi_{D} \ldots \tau^{n} \phi_{D} \tau^{n} V_{D}$ est un $L\left[\left[z-\pi^{q^{n}}\right]\right]$-réseau de $D \otimes_{k((z))} L\left(\left(z-\pi^{q^{n}}\right)\right)$. Pour donner un sens à $v_{q^{n}}\left(\left(\phi_{D}{ }^{\tau} \phi_{D} \cdots \tau^{n-1} \phi_{D}\right)^{-1} x\right)$ on choisit une base de $D$, et cette expression désigne alors le minimum des valuations des coordonnées de $\left(\phi_{D}{ }^{\tau} \phi_{D} \ldots \tau^{n-1} \phi_{D}\right)^{-1} x$ dans la base correspondante de $\tau^{n} D$. Grâce à la constante $C$, la définition de $\widetilde{N}$ est indépendante de ce choix. La proposition suivante permet de reconstruire l'isochtouca local $\left(N, \phi_{N}\right)$ à partir de l'isochtouca de Hodge-Pink associé.

Proposition 7.8. - On $a(N)=\widetilde{N}$.

Démonstration. - C'est un cas particulier de la proposition 3.12. 


\section{Faiblement admissible implique admissible}

Le but de ce paragraphe est d'achever la démonstration du théorème 7.3, en d'autres termes de montrer que le foncteur $\mathbb{D}_{\text {iso }}^{+}$de la catégorie des isochtoucas locaux sur $\theta_{L}$ dans la catégorie $C_{\text {iso-HP-fa }}$ des isochtoucas de Hodge-Pink faiblement admissibles est essentiellement surjectif. On note $C_{i s o-H P-a}$ l'image essentielle de $\mathbb{D}_{\text {iso }}^{+}$, et on appelle isochtoucas de Hodge-Pink admissibles les objets de $C_{i s o-H P-a}$. On commence par énoncer les propositions 8.3 et 8.5. Puis on montrera le théorème 7.3 en admettant les propositions 8.3 et 8.5 et le reste du paragraphe sera consacré à la démonstration des propositions 8.3 et 8.5.

Le lemme suivant est une variante évidente du lemme 2.3 (obtenue en changeant la variable $z$ en $z-\pi$ ).

Lemme 8.1. - Soient $M$ un $\theta \widehat{\otimes} \theta_{L}$-module libre de rang $r$ et $V$ un $L[[z-\pi]]$-réseau de $M \otimes_{\vartheta \widehat{\otimes} \vartheta_{L}} L((z-\pi))$. Alors $M^{\prime}=\left(M \otimes_{\partial \widehat{\otimes} \vartheta_{L}} \oslash \widehat{\otimes} \theta_{L}\left[\frac{1}{z-\pi}\right]\right) \cap V$ est un $\theta \widehat{\otimes} \vartheta_{L}$-module libre et on a $M^{\prime} \otimes_{\vartheta \widehat{\otimes} \vartheta_{L}} \oslash \widehat{\otimes} \vartheta_{L}\left[\frac{1}{z-\pi}\right]=M \otimes_{\partial \widehat{\otimes} \vartheta_{L}} \oslash \widehat{\otimes} \vartheta_{L}\left[\frac{1}{z-\pi}\right]$ et $M^{\prime} \otimes_{\vartheta \widehat{\otimes} \vartheta_{L}} L[[z-\pi]]=V$. Si $V \subset M \otimes_{\widehat{\partial} \widehat{\otimes} \vartheta_{L}} L[[z-\pi]]$ on a $M^{\prime} \subset M$ et en notant $k$ la dimension du $L$-espace vectoriel $M \otimes \widehat{\otimes}_{\vartheta_{L}} L[[z-\pi]] /$ V on a $\operatorname{det}\left(M^{\prime}\right)=(z-\pi)^{k} \operatorname{det}(M)$.

On pourrait aussi modifier $M$ en d'autres diviseurs que $(z-\pi)$ (par exemple $\left(z-\pi^{q^{k}}\right)$ pour $k \in \mathbb{N}$ ) ou en plusieurs diviseurs simultanément (l'ordre n'importe pas par factorialité de $\theta \widehat{\otimes} \theta_{L}$ ). L'application qui à $M$ et $V$ associe $M^{\prime}$ commute aux opérations tensorielles comme le déterminant.

DÉfinition 8.2. - Soit $D=\left(D, \phi_{D}, V_{D}\right)$ un isochtouca de Hodge-Pink tel que $V_{D} \subset U_{D}$.

On définit par récurrence, pour tout entier $n \in \mathbb{N}$, et tout réseau $\Delta$ de $D$ le sous- $\Theta \widehat{\otimes} \theta_{L}$-module $\beta_{n}(\Delta)$ de $D \otimes_{k((z))} \Theta \widehat{\otimes} \vartheta_{L}\left[\frac{1}{z}\right]$ de la façon suivante. On pose $\beta_{0}(\Delta)=\Delta \otimes_{k[[z]]} \theta \widehat{\otimes} \theta_{L}$ et $\beta_{n+1}(\Delta)=\left(\phi_{D} \otimes 1\right)\left({ }^{\tau} \beta_{n}(\Delta) \cap V_{D}\right)$.

Puis on note $\gamma_{n}(\Delta)$ la réduction de $\beta_{n}(\Delta)$ modulo $\pi_{L}$, en d'autres termes, $\gamma_{n}(\Delta)=\beta_{n}(\Delta) \otimes_{\vartheta \widehat{\otimes} \vartheta_{L}} k[[z]]$.

Il résulte du lemme 8.1 que pour tout $n \in \mathbb{N}, \beta_{n}(\Delta)$ est un $\Theta \widehat{\otimes} \vartheta_{L}$-module libre de rang $r$ et

$$
\beta_{n}(\Delta)\left[\frac{1}{z}, \frac{1}{z-\pi}, \ldots, \frac{1}{z-\pi^{q^{n-1}}}\right]=D \otimes_{k((z))} \oslash \widehat{\otimes} \oslash_{L}\left[\frac{1}{z}, \frac{1}{z-\pi}, \ldots, \frac{1}{z-\pi^{q^{n-1}}}\right]
$$

donc $\gamma_{n}(\Delta)$ est un $k[[z]]$-réseau de $D$. Dans la suite on notera $\phi_{D}$ au lieu de $\phi_{D} \otimes 1$.

Proposition 8.3. - Soit $D=\left(D, \phi_{D}, V_{D}\right)$ un isochtouca de Hodge-Pink tel que $V_{D} \subset U_{D}$. Alors les deux assertions suivantes sont équivalentes:

i) pour tout réseau $\Delta$ de $D$ (ou pour un réseau $\Delta$ ) il existe une constante $C$ telle que pour tout $n \in \mathbb{N}$, on a $z^{C} \Delta \subset \gamma_{n}(\Delta) \subset z^{-C} \Delta$.

ii) $D$ est admissible. 
On rappelle que si $\left(D, \phi_{D}\right),\left(D^{\prime}, \phi_{D^{\prime}}\right),\left(D^{\prime \prime}, \phi_{D^{\prime \prime}}\right)$ sont des isochtoucas de Hodge-Pink, une suite exacte courte $0 \rightarrow D^{\prime} \rightarrow D \rightarrow D^{\prime \prime} \rightarrow 0$ dans la catégorie des isochtoucas de Hodge-Pink est une suite exacte dans la catégorie des isochtoucas telle que $V_{D^{\prime \prime}}$ soit l'image de $V_{D}$ dans $D^{\prime \prime} \otimes_{k((z))} L((z-\pi))$ et $V_{D^{\prime}}$ l'intersection de $V_{D}$ avec $D^{\prime} \otimes_{k((z))} L((z-\pi))$. Dans cette situation on a clairement $t_{N}(D)=t_{N}\left(D^{\prime}\right)+t_{N}\left(D^{\prime \prime}\right)$ et $t_{H}(D)=t_{H}\left(D^{\prime}\right)+t_{H}\left(D^{\prime \prime}\right)$. Le lemme suivant est immédiat.

Lemme 8.4. - Les deux assertions suivantes sont équivalentes:

i) D est faiblement admissible et $t_{H}\left(D^{\prime}\right)=t_{N}\left(D^{\prime}\right)$,

ii) $D^{\prime}$ et $D^{\prime \prime}$ sont faiblement admissibles.

Un isochtouca de Hodge-Pink $D=\left(D, \phi_{D}, V_{D}\right)$ faiblement admissible est irréductible dans la catégorie $C_{\text {iso-HP-fa }}$ des isochtoucas de Hodge-Pink faiblement admissibles si et seulement si $t_{N}(D)=t_{H}(D)$ et pour tout sous-isochtouca $D^{\prime}$ autre que 0 et $D$, $t_{H}\left(D^{\prime}\right)<t_{N}\left(D^{\prime}\right)$.

Proposition 8.5. - On suppose $k$ algébriquement clos. Soit $D=\left(D, \phi_{D}, V_{D}\right)$ un objet irréductible dans la catégorie $C_{\text {iso-HP-fa }}$ et tel que $V_{D} \subset U_{D}$.

Alors pour tout réseau $\Delta$ de $D$ (ou de façon équivalente pour un réseau $\Delta$ de $D$ ) il existe une constante $C \in \mathbb{N}$ telle que pour tout $n \in \mathbb{N}$, on a $z^{C} \Delta \subset \gamma_{n}(\Delta) \subset z^{-C} \Delta$.

Démonstration du théorème 7.3 en admettant les propositions 8.3 et 8.5

On a vu au paragraphe précédent que le foncteur $\mathbb{D}_{\text {iso }}^{+}$de la catégorie des isochtoucas locaux sur $\theta_{L}$ dans la catégorie $C_{\text {iso-HP-fa }}$ des isochtoucas de Hodge-Pink faiblement admissibles est pleinement fidèle. Il reste donc à montrer

$(A 1)$ : tout objet $\left(D, \phi_{D}, V_{D}\right)$ dans la catégorie $C_{\text {iso-HP-fa }}$ est admissible.

On considère les énoncés suivants, dont chacun est plus faible que le précédent.

$(A 2)$ : tout objet $\left(D, \phi_{D}, V_{D}\right)$ dans la catégorie $C_{\text {iso-HP-fa }}$ qui vérifie $V_{D} \subset U_{D}$ est admissible. $(A 3)$ : si $k$ est algébriquement clos, tout objet $\left(D, \phi_{D}, V_{D}\right)$ dans la catégorie $C_{\text {iso-HP-fa qui }}$ vérifie $V_{D} \subset U_{D}$ est admissible.

$(A 4)$ : si $k$ est algébriquement clos, tout objet irréductible $\left(D, \phi_{D}, V_{D}\right)$ dans la catégorie $C_{\text {iso-HP-fa }}$ qui vérifie $V_{D} \subset U_{D}$ est admissible.

On va montrer $(A 4)$, puis $(A 3)$, puis $(A 2)$, puis $(A 1)$.

D'abord $(A 4)$ résulte de la proposition 8.5 et de i) $\Rightarrow$ ii) dans la proposition 8.3.

On montre maintenant $(A 3)$ à l'aide de $(A 4)$. Soit $D=\left(D, \phi_{D}, V_{D}\right)$ comme dans $(A 3)$. D'après le lemme 8.4, $D$ admet en tant qu'isochtouca de Hodge-Pink une filtration $0=D_{0} \subset D_{1} \subset \cdots \subset D_{r}=D$ où chaque quotient $D_{i} / D_{i-1}$ est irréductible dans la catégorie $C_{\text {iso-HP-fa }}$ et vérifie $V_{D_{i} / D_{i-1}} \subset U_{D_{i} / D_{i-1}}$. Grâce à $(A 4)$, chaque quotient $D_{i} / D_{i-1}$ est admissible. Or la proposition 3.15 (dans le cas particulier où $S=\operatorname{Spf} \Theta_{L}$ ) montre qu'une extension d'isochtoucas de Hodge-Pink admissibles est admissible et donc $D$ est admissible. On a montré $(A 3)$.

On montre maintenant $(A 2)$ à l'aide de $(A 3)$. Soit $D=\left(D, \phi_{D}, V_{D}\right)$ comme dans $(A 2)$. Le lemme suivant est bien connu des spécialistes. C'est le seul endroit où l'on utilisera que $k$ est parfait. 
Lemme 8.6. - Soient $k$ un corps parfait contenant $\mathbb{F}_{q}$ et $D$ un isochtouca local sur $k$ avec une structure de Hodge-Pink définie sur $L=k\left(\left(\pi_{L}\right)\right)$, et $\bar{D}=D \otimes_{k((z))} \bar{k}((z))$ l'isochtouca local sur $\bar{k}$ avec une structure de Hodge-Pink définie sur $\bar{k}\left(\left(\pi_{L}\right)\right)$ qui s'en déduit (on a par exemple $\left.V_{\bar{D}}=V_{D} \otimes_{k\left(\left(\pi_{L}\right)\right)[[z-\pi]]} \bar{k}\left(\left(\pi_{L}\right)\right)[[z-\pi]]\right)$. Alors si $D$ est faiblement admissible, $\bar{D}$ est faiblement admissible.

Démonstration. - On raisonne par l'absurde. Soit $E \subset \bar{D}$ un sous-isochtouca sur $\bar{k}$ tel que $t_{H}(E)>t_{N}(E)$, et minimal pour cette propriété, ce qui fait que tout quotient $E^{\prime \prime}$ de $E$ vérifie $t_{H}\left(E^{\prime \prime}\right)>t_{N}\left(E^{\prime \prime}\right)$. Alors $D^{\prime}=\sum_{\gamma \in \operatorname{Gal}(\bar{k} / k)} \gamma(E)$ est défini sur $k=\bar{k}^{\operatorname{Gal}(\bar{k} / k)}$, et en tant qu'isochtouca sur $\bar{k}$ est une extension successive de quotients de $\gamma(E)$ pour $\gamma \in \operatorname{Gal}(\bar{k} / k)$, donc vérifie $t_{H}\left(D^{\prime}\right)>t_{N}\left(D^{\prime}\right)$, ce qui contredit l'hypothèse.

Fin de la démonstration du théorème 7.3 en admettant les propositions 8.3 et 8.5

Grâce au lemme 8.6, $\bar{D}=D \otimes_{k((z))} \bar{k}((z))$ est faiblement admissible. Grâce à $(A 3)$, $\bar{D}$ est admissible en tant qu'isochtouca de Hodge-Pink sur $\bar{k}$ (c'est-à-dire qu'il est associé à un isochtouca local sur $\left.\bar{k}\left[\left[\pi_{L}\right]\right]\right)$. Par ii) $\Rightarrow$ i) de la proposition 8.3 il satisfait la conclusion i) de la proposition 8.3. Il en résulte immédiatement que $D$ satisfait la conclusion i) de la proposition 8.3 et par i) $\Rightarrow$ ii) de la proposition $8.3, D$ est admissible. On a montré $(A 2)$.

Enfin $(A 1)$ résulte de $(A 2)$. En effet, on s'y ramène en multipliant $\phi_{D}$ par $z^{-k}$ et $V_{D}$ par $(z-\pi)^{k}$ pour un entier $k$ assez grand (cela ne change pas la faible admissibilité, et si $\left(D, z^{-k} \phi_{D},(z-\pi)^{k} V_{D}\right)$ est associé à un isochtouca local $\left(N, \phi_{N}\right),\left(D, \phi_{D}, V_{D}\right)$ est associé à $\left.\left(N,(z-\pi)^{k} \phi_{N}\right)\right)$. On a terminé la démonstration du théorème 7.3.

Nous passons maintenant à la démonstration des propositions 8.3 et 8.5. Nous commençons par des propriétés générales de $\beta_{n}$ et $\gamma_{n}$. Regardons déjà ce qui se passe quand $r=\operatorname{dim} V$ vaut 1 . Plus précisément prenons $t_{H} \in \mathbb{Z}_{-}, t_{N} \in \mathbb{Z}$ et posons $D=k((z)), \quad \phi_{D}=z^{t_{N}}, \quad V_{D}=(z-\pi)^{-t_{H}} U_{D} \quad$ et $\quad \Delta=k[[z]] . \quad$ On a alors $\beta_{n}(\Delta)=z^{n\left(t_{N}-t_{H}\right)}\left(\left(1-\frac{\pi}{z}\right)\left(1-\frac{\pi^{q}}{z}\right) \cdots\left(1-\frac{\pi^{q^{n-1}}}{z}\right)\right)^{-t_{H}} \oslash \widehat{\otimes} \vartheta_{L}$ et $\gamma_{n}(\Delta)=z^{n\left(t_{N}-t_{H}\right)} k[[z]]$. On voit que la condition i) de la proposition 8.3 est vérifiée si et seulement si $t_{N}=t_{H}$. On suppose $t_{N}=t_{H}=t \in \mathbb{Z}_{-}$. Alors $\left(D, \phi_{D}, V_{D}\right)$ est admissible (comme le prévoit la proposition 8.3) et il est associé à l'isochtouca local $\left(N, \phi_{N}\right)=\left(\Theta \widehat{\otimes} \theta_{L}\left[\frac{1}{z}\right],(z-\pi)^{t}\right)$. Si on note $M=\emptyset \widehat{\otimes} \theta_{L} \subset N$ on a $R(M)=\alpha^{-t} \oslash \widehat{\otimes} \theta_{L}=\tau^{n} \alpha^{-t} \beta_{n}(\Delta)$. On voit que la suite $\beta_{n}(\Delta)$ « converge » vers $R(M)$ car $\tau^{n} \alpha$ est une unité dans $\Theta \widehat{\otimes} \vartheta_{L}\left[\left[\frac{\pi^{q^{n}}}{z}\right]\right]$. Plus généralement pour tout $\oslash \widehat{\otimes} \theta_{L}$-module $M$ libre de rang 1 muni d'un isomorphisme $M\left[\frac{1}{z}\right]=N$ il existe $C$ tel que pour tout $n$ on ait

$$
z^{C} \beta_{n}(\Delta)\left[\left[\frac{\pi^{q^{n}}}{z}\right]\right] \subset R(M)\left[\left[\frac{\pi^{q^{n}}}{z}\right]\right] \subset z^{-C} \beta_{n}(\Delta)\left[\left[\frac{\pi^{q^{n}}}{z}\right]\right] .
$$

Dans la formule précédente on a utilisé la convention suivante, qui servira dans toute la suite : si $Q$ est un $\theta \widehat{\otimes} \vartheta_{L}$-module libre on note $Q\left[\left[\frac{\pi^{q^{n}}}{z}\right]\right]=Q \otimes_{\vartheta \widehat{\otimes} \vartheta_{L}} \theta \widehat{\otimes} \vartheta_{L}\left[\left[\frac{\pi^{q^{n}}}{z}\right]\right]$. Les inclusions (49) auront lieu pour tout isochtouca local $\left(N, \phi_{N}\right)$ et joueront un rôle important dans la preuve de la proposition 8.3.

Le lemme suivant établit quelques propriétés satisfaites $\operatorname{par} \beta_{n}(\Delta)$ et $\gamma_{n}(\Delta)$.

Lemme 8.7. - Soient $\left(D, \phi_{D}, V_{D}\right)$ et $\Delta$ comme dans la définition 8.2. On suppose $t_{N}(D)=t_{H}(D)$. Alors il existe une constante $C$ telle que pour tout $n \in \mathbb{N}$, 
a) $\beta_{n+1}(\Delta) \subset\left(\phi_{D} \otimes 1\right)\left({ }^{\tau} \beta_{n}(\Delta)\right)$ et $\beta_{n+1}(\Delta) \subset z^{-C} \beta_{n}(\Delta)$,

b) les réseaux $\operatorname{det}\left(\gamma_{n}(\Delta)\right)$ et $\operatorname{det}(\Delta)$ dans $\operatorname{det}(D)$ sont égaux et deux réseaux de $D$ quelconques $U$ et $U^{\prime}$ parmi $\gamma_{n}(\Delta), \gamma_{n+1}(\Delta), \phi_{D}\left({ }^{\tau} \gamma_{n}(\Delta)\right), \phi_{D}\left({ }^{\tau} \gamma_{n+1}(\Delta)\right)$ vérifient $U \subset z^{-C} U^{\prime}$

c) pour tout entier $k \in \mathbb{N}$,

$$
\beta_{n}(\Delta) \bmod \pi_{L}^{q^{k}} \subset z^{-k C} \gamma_{n}(\Delta) \otimes_{k[[z]]} \oslash \widehat{\otimes} \theta_{L} \bmod \pi_{L}^{q^{k}},
$$

d) on $a$

$$
z^{C} \gamma_{n}(\Delta) \otimes_{k[[z]]} \oslash \widehat{\otimes} \theta_{L}\left[\left[\frac{\pi}{z}\right]\right] \subset \beta_{n}(\Delta)\left[\left[\frac{\pi}{z}\right]\right] \subset z^{-C} \gamma_{n}(\Delta) \otimes_{k[[z]]} \oslash \widehat{\otimes}_{L}\left[\left[\frac{\pi}{z}\right]\right],
$$

e) pour tout $n^{\prime} \in \mathbb{N}$ on a

$$
z^{C} \gamma_{n+n^{\prime}}(\Delta) \subset \gamma_{n} \circ \gamma_{n^{\prime}}(\Delta) \subset z^{-C} \gamma_{n+n^{\prime}}(\Delta) .
$$

Démonstration. - D'abord l'inclusion évidente $\beta_{n+1}(\Delta) \subset\left(\phi_{D} \otimes 1\right)\left({ }^{\tau} \beta_{n}(\Delta)\right)$ implique $\gamma_{n+1}(\Delta) \subset \phi_{D}\left({ }^{\tau} \gamma_{n}(\Delta)\right)$. Il existe $C \in \mathbb{N}$ tel que $\phi_{D}\left({ }^{\tau} \Delta\right) \subset z^{-C} \Delta$. Cela implique $\beta_{1}(\Delta) \subset z^{-C} \beta_{0}(\Delta)$. On a alors, pour tout $n, \beta_{n+1}(\Delta) \subset z^{-C} \beta_{n}(\Delta)$, ce qui termine la preuve de a). On en déduit pour tout $n \in \mathbb{N}, \gamma_{n+1}(\Delta) \subset z^{-C} \gamma_{n}(\Delta)$. La construction de $\beta_{n}(\Delta)$ et de $\gamma_{n}(\Delta)$ commute au déterminant : le fait que l'intersection avec $V_{D}$ commute au déterminant, et en fait à toute opération tensorielle, n'est pas évident, c'est une conséquence de la remarque après le lemme 8.1. On a donc

$$
\operatorname{det}\left(\beta_{n}(\Delta)\right)=\left(\left(1-\frac{\pi}{z}\right)\left(1-\frac{\pi^{q}}{z}\right) \cdots\left(1-\frac{\pi^{q^{n-1}}}{z}\right)\right)^{-t_{N}(D)} \operatorname{det}(\Delta) \otimes_{k((z))} \theta \widehat{\otimes} \theta_{L}
$$

comme sous- $\Theta \widehat{\otimes} \vartheta_{L}$-module $\operatorname{de} \operatorname{det}(D) \otimes_{k((z))} \theta \widehat{\otimes} \Theta_{L}\left[\frac{1}{z}\right]$, et $\operatorname{donc} \operatorname{det}\left(\gamma_{n}(\Delta)\right)=\operatorname{det}(\Delta)$ comme $k[[z]]$-réseau de $\operatorname{det}(D)$. Les inclusions $\gamma_{n+1}(\Delta) \subset \phi_{D}\left({ }^{\tau} \gamma_{n}(\Delta)\right)$ et $\gamma_{n+1}(\Delta) \subset z^{-C} \gamma_{n}(\Delta)$ et l'égalité $\operatorname{det}\left(\gamma_{n}(\Delta)\right)=\operatorname{det}(\Delta)$ montrent qu'il existe une constante $C$ indépendante de $n$ telle que la propriété b) du lemme soit vraie.

On déduit de l'inclusion évidente $\beta_{n+1}(\Delta) \subset\left(\phi_{D} \otimes 1\right)\left({ }^{\tau} \beta_{n}(\Delta)\right)$ que pour tout $n$ on a

$$
\begin{aligned}
& \beta_{n}(\Delta) \bmod \pi_{L}^{q} \subset \phi_{D}\left({ }^{\tau} \gamma_{n-1}(\Delta)\right) \otimes_{k[[z]]} \oslash \widehat{\otimes} \theta_{L} \bmod \pi_{L}^{q}, \\
& \beta_{n}(\Delta) \bmod \pi_{L}^{q^{2}} \subset \phi_{D}{ }^{\tau} \phi_{D}\left({ }^{2} \gamma_{n-2}(\Delta)\right) \otimes_{k[[z]]} \oslash \widehat{\otimes} \vartheta_{L} \bmod \pi_{L}^{q^{2}} \cdots
\end{aligned}
$$

Grâce à b), la propriété c) en découle.

Par la propriété c) il existe une constante $C$ indépendante de $n$ telle que

$$
\beta_{n}(\Delta) \subset z^{-C} \gamma_{n}(\Delta) \otimes_{k[[z]]} \ominus \widehat{\otimes} \theta_{L}\left[\left[\frac{\pi}{z}\right]\right] .
$$

Mais le déterminant de $\beta_{n}(\Delta)$ et celui de $\gamma_{n}(\Delta)$ diffèrent par

$$
\left(\left(1-\frac{\pi}{z}\right)\left(1-\frac{\pi^{q}}{z}\right) \cdots\left(1-\frac{\pi^{q^{n-1}}}{z}\right)\right)^{t_{N}(D)}
$$

qui est une unité de $\theta \widehat{\otimes} \theta_{L}\left[\left[\frac{\pi}{z}\right]\right]$. On en déduit qu'il existe une constante $C$ indépendante de $n$ telle que d) soit vrai.

La propriété e) est plus difficile à démontrer. Elle jouera un grand rôle dans la démonstration de la proposition 8.5. Bien sûr il suffit de montrer l'une des deux inclusions car les réseaux $\gamma_{n} \circ \gamma_{n^{\prime}}(\Delta)$ et $\gamma_{n+n^{\prime}}(\Delta)$ ont des déterminants égaux. C'est l'inclusion de droite que nous allons démontrer. 
On a

(50)

$$
\begin{aligned}
\beta_{n}\left(\gamma_{n^{\prime}}(\Delta)\right)=\phi_{D} \cdots \tau^{n-1} \phi_{D}\left(\tau^{n} \gamma_{n^{\prime}}(\Delta) \otimes_{k[[z]]} \otimes \widehat{\otimes} \theta_{L}\right) \\
\\
\cap\left(\phi_{D} \cdots \tau^{n-1} \phi_{D}{ }^{\tau^{n-1}} V_{D} \oplus \cdots \oplus \phi_{D} V_{D}\right) .
\end{aligned}
$$

Dans cette formule $\tau^{\tau^{i}} V_{D}$ est un $L\left[\left[z-\pi^{q^{i}}\right]\right]$-réseau de $\tau^{\tau^{i+1}} D \otimes_{k((z))} L\left(\left(z-\pi^{q^{i}}\right)\right)$ et l'intersection a lieu dans $D \otimes L\left[\left[z-\pi^{q^{n-1}}\right]\right] \oplus \cdots \oplus D \otimes L[[z-\pi]]$ où $\phi_{D} \ldots \tau^{n-1} \phi_{D}\left(\tau^{n} \gamma_{n^{\prime}}(\Delta) \otimes_{k[[z]]} \oslash \widehat{\otimes} \vartheta_{L}\right)$ s'envoie diagonalement. D'après $\mathrm{d}$ ) on a

$$
\tau^{n} \gamma_{n^{\prime}}(\Delta) \otimes_{k[[z]]} \oslash \widehat{\otimes} \theta_{L} \subset z^{-C \tau^{n}} \beta_{n^{\prime}}(\Delta)\left[\left[\frac{\pi^{q^{n}}}{z}\right]\right]
$$

et (50) implique alors

$\beta_{n}\left(\gamma_{n^{\prime}}(\Delta)\right) \subset z^{-C} \phi_{D} \ldots \tau^{n-1} \phi_{D}\left(\tau^{n} \beta_{n^{\prime}}(\Delta)\left[\left[\frac{\pi^{q^{n}}}{z}\right]\right]\right) \cap\left(\phi_{D} \ldots \tau^{\tau^{n-1}} \phi_{D} \tau^{n-1} V_{D} \oplus \cdots \oplus \phi_{D} V_{D}\right)$.

On rappelle que $s \in \mathbb{Z}_{-}$est tel que $V_{D} \supset(z-\pi)^{-s} U_{D}$. On applique le lemme 8.8 ci-dessous à

$$
\mathcal{M}=\phi_{D} \ldots{ }^{n-1} \phi_{D}\left({ }^{\tau^{n}} \beta_{n^{\prime}}(\Delta)\right), V_{0}=\phi_{D} V_{D}, \ldots, V_{n-1}=\phi_{D} \ldots \tau^{n-1} \phi_{D} \tau^{n-1} V_{D}
$$

Comme $\mathcal{M} \cap\left(V_{0} \oplus \cdots \oplus V_{n-1}\right)=\beta_{n+n^{\prime}}(\Delta)$ le lemme 8.8 implique

$$
\left(\mathcal{M}\left[\left[\frac{\pi^{q^{n}}}{z}\right]\right]\right) \cap\left(V_{0} \oplus \cdots \oplus V_{n-1}\right) \subset z^{-C_{1}} \beta_{n+n^{\prime}}(\Delta)\left[\left[\frac{\pi^{q^{n}}}{z}\right]\right] .
$$

L’inclusion (52) se réécrit

$$
\beta_{n}\left(\gamma_{n^{\prime}}(\Delta)\right) \subset z^{-C}\left(\mathcal{M}\left[\left[\frac{\pi^{q^{n}}}{z}\right]\right]\right) \cap\left(\mathcal{V}_{0} \oplus \cdots \oplus \mathcal{V}_{n-1}\right)
$$

Les inclusions (53) et (54) impliquent

$$
\begin{gathered}
\beta_{n}\left(\gamma_{n^{\prime}}(\Delta)\right) \subset z^{-C-C_{1}} \beta_{n+n^{\prime}}(\Delta)\left[\left[\frac{\pi^{q^{n}}}{z}\right]\right] \\
\text { d'où } \gamma_{n}\left(\gamma_{n^{\prime}}(\Delta)\right)=\beta_{n}\left(\gamma_{n^{\prime}}(\Delta)\right) \bmod \pi_{L} \subset z^{-C-C_{1}} \gamma_{n+n^{\prime}}(\Delta),
\end{gathered}
$$

ce qui achève la démonstration du lemme 8.7.

Lemme 8.8. - Pour tout $s \in \mathbb{Z}_{-}$il existe une constante $C_{1}$ telle que l'énoncé suivant soit vrai. Soient $n \in \mathbb{N}, \mathcal{M}$ un $\oslash \widehat{\otimes} \vartheta_{L}$-module libre de rang $r$, et pour $i \in\{0, \ldots, n-1\}$ soit $V_{i}$ un $L\left[\left[z-\pi^{q^{i}}\right]\right]$-module libre de rang $r$ vérifiant

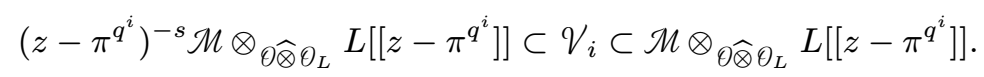

Alors $\mathcal{M} \cap\left(V_{0} \oplus \cdots \oplus V_{n-1}\right)$ est un $\oslash \widehat{\otimes} \vartheta_{L}$-module libre de rang $r$ et on a

$$
\begin{aligned}
\left(\mathcal{M} \cap\left(V_{0} \oplus \cdots \oplus V_{n-1}\right)\right)\left[\left[\frac{\pi^{q^{n}}}{z}\right]\right] \subset(\mathcal{M}[ & {\left.\left.\left[\frac{\pi^{q^{n}}}{z}\right]\right]\right) \cap\left(V_{0} \oplus \cdots \oplus V_{n-1}\right) } \\
& \subset z^{-C_{1}}\left(\mathcal{M} \cap\left(V_{0} \oplus \cdots \oplus V_{n-1}\right)\right)\left[\left[\frac{\pi^{q^{n}}}{z}\right]\right] .
\end{aligned}
$$


Démonstration. - D'après le lemme $8.1, \check{M}=M \cap\left(V_{0} \oplus \cdots \oplus V_{n-1}\right)$ est un $\oslash \widehat{\otimes} \theta_{L}$-module libre de rang $r$ et

$$
V_{i}=\check{M} \otimes_{\partial \widehat{\otimes} \vartheta_{L}} L\left[\left[z-\pi^{q^{i}}\right]\right] \text { pour tout } i \in\{0, \ldots, n-1\} .
$$

La première inclusion de (55) est évidente et pour la deuxième on remarque que, grâce à (56),

$$
\begin{aligned}
& \left(\mathcal{M}\left[\left[\frac{\pi^{q^{n}}}{z}\right]\right]\right) \cap\left(V_{0} \oplus \cdots \oplus V_{n-1}\right) \subset \check{M} \otimes_{\partial \widehat{\otimes} \vartheta_{L}} \\
& \quad\left(\left(\left((z-\pi) \cdots\left(z-\pi^{q^{n-1}}\right)\right)^{s} \oslash \widehat{\otimes} \vartheta_{L}\left[\left[\frac{\pi^{q^{n}}}{z}\right]\right]\right) \cap\left(L\left[\left[z-\pi^{q^{n-1}}\right]\right] \oplus \cdots \oplus L[[z-\pi]]\right)\right)
\end{aligned}
$$

et on applique le lemme suivant.

Lemme 8.9. - Pour tout $s \in \mathbb{Z}_{-}$il existe une constante $C_{1}$, telle que pour tout $n \in \mathbb{N}$,

$$
\begin{aligned}
\left(\left((z-\pi) \cdots\left(z-\pi^{q^{n-1}}\right)\right)^{s} \oslash \widehat{\otimes} \theta_{L}\left[\left[\frac{\pi^{q^{n}}}{z}\right]\right]\right) \cap\left(L\left[\left[z-\pi^{q^{n-1}}\right]\right]\right. & \oplus \cdots \oplus[[z-\pi]]) \\
& \subset z^{-C_{1}} \oslash \widehat{\otimes} \vartheta_{L}\left[\left[\frac{\pi^{q^{n}}}{z}\right]\right] .
\end{aligned}
$$

Démonstration. - On prend pour $C_{1}$ le plus petit entier supérieur ou égal à $1+(-s)\left(\frac{1}{q}+\frac{1}{q^{2}}+\cdots\right)$. Une adaptation facile de la preuve du a) du lemme 3.5 montre que

$$
\begin{aligned}
\left(\left((z-\pi) \cdots\left(z-\pi^{q^{n-1}}\right)\right)^{s} \oslash \widehat{\otimes} \Theta_{L}\left[\left[\frac{\pi^{q^{n}}}{z}\right]\right]\right) \cap\left(L\left[\left[z-\pi^{q^{n-1}}\right]\right] \oplus\right. & \cdots \oplus L[[z-\pi]]) \\
& \subset \oslash \widehat{\otimes} \vartheta_{L}\left[\left[\frac{\pi^{q^{n}}}{z}\right]\right]\left[\frac{1}{z}\right] .
\end{aligned}
$$

En effet pour tout $i \in\{0, \ldots, n-1\}$ on montre

$$
\left(z-\pi^{q^{i}}\right)^{s} \Theta \widehat{\otimes} \vartheta_{L}\left[\left[\frac{\pi^{q^{n}}}{z}\right]\right]\left[\frac{1}{z}\right] \cap L\left[\left[z-\pi^{q^{i}}\right]\right]=\Theta \widehat{\otimes} \vartheta_{L}\left[\left[\frac{\pi^{q^{n}}}{z}\right]\right]\left[\frac{1}{z}\right]
$$

en utilisant (4) avec $i$ au lieu de $n$. On met sur $\Theta \widehat{\otimes} \vartheta_{L}\left[\left[\frac{\pi^{q^{n}}}{z}\right]\right]\left[\frac{1}{z}\right]$ la valuation $v_{q^{n}}$ donnée par

$$
v_{q^{n}}\left(\sum_{i, j} x_{i, j} z^{i} \pi_{L}^{j}\right)=\inf _{x_{i, j} \neq 0} q^{n} i+\frac{j}{e} .
$$

On a alors pour tout entier $r \in \mathbb{Z}$,

$$
z^{r} \Theta \widehat{\otimes} \vartheta_{L}\left[\left[\frac{\pi^{q^{n}}}{z}\right]\right] \subset\left\{x \in \Theta \widehat{\otimes} \vartheta_{L}\left[\left[\frac{\pi^{q^{n}}}{z}\right]\right]\left[\frac{1}{z}\right], v_{q^{n}}(x) \geq q^{n} r\right\} \subset z^{r-1} \Theta \widehat{\otimes} \vartheta_{L}\left[\left[\frac{\pi^{q^{n}}}{z}\right]\right] .
$$

En effet $x=\sum_{i, j} x_{i, j} z^{i} \pi_{L}^{j}$ appartient à $z^{r} \oslash \widehat{\otimes} \vartheta_{L}\left[\left[\frac{\pi^{q^{n}}}{z}\right]\right]$ (resp. vérifie $v_{q^{n}}(x) \geq q^{n} r$ ) si et seulement si les $(i, j)$ tels que $x_{i, j} \neq 0$ vérifient $i \geq r-\left[\frac{j}{e q^{n}}\right]$ (resp. vérifient $i \geq r-\frac{j}{e q^{n}}$ ) et on a $\left[\frac{j}{e q^{n}}\right] \leq \frac{j}{e q^{n}} \leq\left[\frac{j}{e q^{n}}\right]+1$. Comme $v_{q^{n}}$ est une valuation et $v_{q^{n}}(z-\pi)=1, \ldots, v_{q^{n}}\left(z-\pi^{q^{n-1}}\right)=q^{n-1}$, le lemme en résulte.

Démonstration de «ii) implique i) » dans la proposition 8.3. - Soit $\left(M, \phi_{M}\right)$ un chtouca local dont l'isochtouca de Hodge-Pink associé est $D=\left(D, \phi_{D}, V_{D}\right)$. Pour vérifier i) choisissons $\Delta=M \bmod \pi_{L}$. Comme $\mathscr{C}\left[\frac{1}{\alpha}\right] \subset \vartheta \widehat{\otimes} \vartheta_{L}\left[\left[\frac{\pi}{z}\right]\right]\left[\frac{1}{z}\right]$, le lemme 7.4 montre qu'il existe $C$ tel que $R(M) \subset z^{-C} \beta_{0}(\Delta)\left[\left[\frac{\pi}{z}\right]\right]$. Quitte à augmenter $C$ on suppose de plus que l'énoncé 
du lemme 8.7 est vrai pour cette valeur de $C$ (le lemme 8.7 s'applique car $t_{N}(D)=t_{H}(D)$ puisque $D$ est admissible).

On en déduit $\tau^{n} R(M) \subset z^{-C \tau^{n}} \beta_{0}(\Delta)\left[\left[\frac{\pi^{q^{n}}}{z}\right]\right]$. Par factorialité de $\Theta \widehat{\otimes} \vartheta_{L}$ on a

$$
M=\phi_{M}\left({ }^{\tau} M \cap\left(\phi_{M}^{-1}(M) \otimes_{\vartheta \widehat{\otimes} \vartheta_{L}} L[[z-\pi]]\right)\right) .
$$

Autrement dit $R(M)=\phi_{D}\left({ }^{\tau}(R(M)) \cap V_{D}\right)$ d'où

$$
R(M) \subset z^{-C} \phi_{D} \ldots \tau^{n-1} \phi_{D}\left(\tau^{n} \beta_{0}(\Delta)\right)\left[\left[\frac{\pi^{q^{n}}}{z}\right]\right] \cap\left(\phi_{D} \ldots \tau^{\tau^{n-1}} \phi_{D} \tau^{n-1} V_{D} \oplus \cdots \oplus \phi_{D} V_{D}\right)
$$

On applique le lemme 8.8 à

$$
\mathcal{M}=\phi_{D}{ }^{\tau} \phi_{D} \cdots{ }^{\tau^{n-1}} \phi_{D}\left(\tau^{n} \beta_{0}(\Delta)\right), V_{0}=\phi_{D} V_{D}, \ldots, V_{n-1}=\phi_{D} \cdots^{\tau^{n-1}} \phi_{D}^{\tau^{n-1}} V_{D}
$$

L'inclusion (57) se réécrit

$$
R(M) \subset z^{-C}\left(\mathcal{M}\left[\left[\frac{\pi^{q^{n}}}{z}\right]\right]\right) \cap\left(V_{0} \oplus \cdots \oplus V_{n-1}\right)
$$

et par définition de $\beta_{n}(\Delta)$ on a

$$
\beta_{n}(\Delta)=\mathcal{M} \cap\left(V_{0} \oplus \cdots \oplus V_{n-1}\right)
$$

Donc le lemme 8.8 implique

$$
R(M) \subset z^{-C-C_{1}} \beta_{n}(\Delta)\left[\left[\frac{\pi^{q^{n}}}{z}\right]\right] .
$$

On en déduit $\Delta=R(M) \bmod \pi_{L} \subset z^{-C-C_{1}} \gamma_{n}(\Delta)$. Ceci montre l'une des inclusions de i) de la proposition 8.3, mais l'autre en résulte car $t_{N}(D)=t_{H}(D)$ et $\operatorname{donc} \operatorname{det}\left(\gamma_{n}(\Delta)\right)=\operatorname{det}(\Delta)$.

Remarque. - L'inclusion (58) et un calcul de déterminants montrent qu'il existe $C$ tel que pour tout $n \in \mathbb{N}$ on ait

$$
z^{C} \beta_{n}(\Delta)\left[\left[\frac{\pi^{q^{n}}}{z}\right]\right] \subset R(M)\left[\left[\frac{\pi^{q^{n}}}{z}\right]\right] \subset z^{-C} \beta_{n}(\Delta)\left[\left[\frac{\pi^{q^{n}}}{z}\right]\right] .
$$

On pourrait en déduire une autre preuve de la proposition 7.5 « admissible implique faiblement admissible». Mais le plus important est que (59) justifie a posteriori la construction de l'isochtouca $\left(N, \phi_{N}\right)$ dans la preuve de «i) implique ii)» que nous allons donner maintenant.

Démonstration de «i $\Rightarrow$ ii) » dans la proposition 8.3. - On a $\operatorname{det}\left(\gamma_{n}(\Delta)\right)=z^{n\left(t_{N}(D)-t_{H}(D)\right)}$. La condition i) entraîne que $t_{N}(D)=t_{H}(D)$, ce qui permet d'appliquer le lemme 8.7. On fixe un réseau $\Delta$ de $D$.

Lemme 8.10. - Sous la condition i) de la proposition 8.3 il existe $C$ tel que pour tous les entiers $m, n \in \mathbb{N}$ avec $n \geq m$, on ait

$$
\begin{aligned}
z^{C} \beta_{m}(\Delta)\left[\left[\frac{\pi^{q^{m}}}{z}\right]\right] & \subset \beta_{n}(\Delta)\left[\left[\frac{\pi^{q^{m}}}{z}\right]\right] \subset z^{-C} \beta_{m}(\Delta)\left[\left[\frac{\pi^{q^{m}}}{z}\right]\right] \\
\text { et } z^{C} \beta_{m}(\Delta) \bmod \pi^{q^{m}} & \subset \beta_{n}(\Delta) \bmod \pi^{q^{m}} \subset z^{-C} \beta_{m}(\Delta) \bmod \pi^{q^{m}} .
\end{aligned}
$$


Démonstration. - Les trois termes de (61) sont des $\theta \widehat{\otimes}\left(\theta_{L} / \pi^{q^{m}}\right)$-modules libres de rang $r$ dans $D \otimes_{k((z))} \Theta \widehat{\otimes}\left(\theta_{L} / \pi^{q^{m}}\right)\left[\frac{1}{z}\right]$. La suite d'inclusions (61) résulte de (60) par réduction modulo l'idéal de $\Theta \widehat{\otimes} \vartheta_{L}\left[\left[\frac{\pi^{q^{m}}}{z}\right]\right]$ engendré par $\frac{\pi^{q^{m}}}{z}$. Pour montrer l'inégalité de droite de (60) on applique le lemme 8.8 avec $m$ à la place de $n$ et

$$
\mathcal{M}=\phi_{D} \ldots \tau^{m-1} \phi_{D}\left(\tau^{m} \beta_{0}(\Delta)\right), V_{0}=\phi_{D} V_{D}, \ldots, V_{m-1}=\phi_{D} \ldots \tau^{m-1} \phi_{D} \tau^{m-1} V_{D}
$$

On a

$$
\begin{aligned}
\beta_{m}(\Delta) & =\mathcal{M} \cap\left(V_{0} \oplus \cdots \oplus V_{m-1}\right) \\
\text { et } \quad \beta_{n}(\Delta) & =\phi_{D} \ldots \tau^{\tau^{m-1}} \phi_{D}\left({ }^{\tau^{m}} \beta_{n-m}(\Delta)\right) \cap\left(V_{0} \oplus \cdots \oplus \mathcal{V}_{m-1}\right) .
\end{aligned}
$$

Or

$$
\begin{aligned}
\beta_{n-m}(\Delta) & \subset z^{-C} \gamma_{n-m}(\Delta) \otimes \ominus \widehat{\otimes} \vartheta_{L}\left[\left[\frac{\pi}{z}\right]\right] \\
& \subset z^{-C-C^{\prime}} \gamma_{0}(\Delta) \otimes \ominus \widehat{\otimes} \theta_{L}\left[\left[\frac{\pi}{z}\right]\right]=z^{-C-C^{\prime}} \beta_{0}(\Delta)\left[\left[\frac{\pi}{z}\right]\right]
\end{aligned}
$$

grâce à la propriété d) du lemme 8.7 et à la condition i) de la proposition 8.3 (en notant $C^{\prime}$ la constante qui y apparaît). D'où

$$
\tau^{m} \beta_{n-m}(\Delta) \subset z^{-C-C^{\prime} \tau^{m}} \beta_{0}(\Delta)\left[\left[\frac{\pi^{q^{m}}}{z}\right]\right]
$$

et par (63) on a donc

$$
\beta_{n}(\Delta) \subset z^{-C-C^{\prime}} \mathcal{M}\left[\left[\frac{\pi^{q^{m}}}{z}\right]\right] \cap\left(V_{0} \oplus \cdots \oplus V_{m-1}\right)
$$

Grâce à (62) et (64) le lemme 8.8 implique

$$
\beta_{n}(\Delta) \subset z^{-C-C^{\prime}-C_{1}} \beta_{m}(\Delta)\left[\left[\frac{\pi^{q^{m}}}{z}\right]\right]
$$

Donc on a établi l'inégalité de droite de (60), et un calcul de déterminants permet d'en déduire l'inégalité de gauche.

Suite de la démonstration de «i) implique ii) » dans la proposition 8.3

Notons $\mathscr{C}$ l'anneau de valuation discrète de corps résiduel $k((z))$ qui est le complété de $\theta \widehat{\otimes} \theta_{L}\left[\frac{1}{z}\right]$ pour la topologie $\pi$-adique. C'est $\mathscr{G}\left(\theta_{L}\right)$ dans les notations du paragraphe 3 . On a $\mathscr{G} / \pi^{q^{m}} \mathscr{G}=\theta \widehat{\otimes}\left(\theta_{L} / \pi^{q^{m}}\right)\left[\frac{1}{z}\right]$. On pose alors

$$
N=\left\{x \in D \otimes_{k((z))} \mathscr{G}, \exists k \in \mathbb{Z}, \forall m \in \mathbb{N}, x \bmod \pi^{q^{m}} \in z^{k} \beta_{m}(\Delta) \bmod \pi^{q^{m}}\right\}
$$

et on définit $\phi_{N}^{-1}: N \rightarrow{ }^{\tau} N$ comme la restriction de

$$
\phi_{D}^{-1}: D \otimes_{k((z))} \mathscr{C} \rightarrow{ }^{\tau} D \otimes_{k((z))} \mathscr{G}
$$

(a posteriori comme $V_{D} \subset U_{D}, \phi_{N}^{-1}$ n'a pas de dénominateur en $z-\pi$, la matrice $R$ associée à $N$ est à coefficients dans $\mathscr{C}$ au lieu de $\mathscr{C}\left[\frac{1}{\alpha}\right]$ et $\left.N \subset D \otimes_{k((z))} \mathscr{C} \subset D \otimes_{k((z))} \mathscr{C}\right)$.

Il nous reste à montrer que $N$ est un $\oslash \widehat{\otimes} \Theta_{L}\left[\frac{1}{z}\right]$-module libre de rang $r$, que $\phi_{N}^{-1}: N \rightarrow{ }^{\tau} N$ est bien défini et détermine un isomorphisme de $\theta \widehat{\otimes} \theta_{L}\left[\frac{1}{z}, \frac{1}{z-\pi}\right]$-modules $\phi_{N}:{ }^{\tau}\left[\frac{1}{z-\pi}\right] \rightarrow N\left[\frac{1}{z-\pi}\right]$, que $\left(N, \phi_{N}\right)$ est un isochtouca local et que $D=\left(D, \phi_{D}, V_{D}\right)$ est l'isochtouca de Hodge-Pink associé. Le lemme général suivant, appliqué à $Q_{m}=\beta_{m}(\Delta) \otimes_{\vartheta \widehat{\otimes} \vartheta_{L}} \oslash \widehat{\otimes} \vartheta_{L} / \pi^{q^{m}}$ montre que $N$ est un $\vartheta \widehat{\otimes} \vartheta_{L}\left[\frac{1}{z}\right]$-module libre. Les conditions du lemme suivant sont satisfaites grâce à (61) dans le lemme 8.10. 
Lemme 8.11. - a) Soit $P$ un $\mathscr{G}$-module libre de rang $r$ et, pour tout $m \in \mathbb{N}$, soit $Q_{m}$ un sous$\emptyset \widehat{\otimes} \vartheta_{L} / \pi^{q^{m}}$-module libre de rang $r$ de $P / \pi^{q^{m}} P$ tel que $Q_{m} \otimes_{\vartheta \widehat{\otimes} \vartheta_{L} / \pi^{q^{m}}} \mathscr{G} / \pi^{q^{m}} \mathscr{G}=P / \pi^{q^{m}} P$. On suppose qu'il existe $C \in \mathbb{N}$, tel que pour $m, n \in \mathbb{N}$, avec $n \geq m$, en notant $Q_{n} \bmod \pi^{q^{m}}$ l'image de $Q_{n}$ dans $P / \pi^{q^{m}} P$, on ait

$$
z^{C} Q_{m} \subset Q_{n} \bmod \pi^{q^{m}} \subset z^{-C} Q_{m}
$$

Alors

$$
N=\left\{x \in P, \exists k \in \mathbb{Z}, \forall m \in \mathbb{N}, x \bmod \pi^{q^{m}} \in z^{k} Q_{m}\right\}
$$

est un $\theta \widehat{\otimes} \theta_{L}\left[\frac{1}{z}\right]-$ module libre de rang $r$ muni naturellement d'un isomorphisme $N \otimes_{\oslash \widehat{\otimes} \vartheta_{L}\left[\frac{1}{z}\right]} \mathscr{A}=P$ et si $M$ est un $\oslash \widehat{\otimes} \vartheta_{L}$-module libre tel que $M \otimes_{\vartheta \widehat{\otimes} \theta_{L}} \oslash \widehat{\otimes} \vartheta_{L}\left[\frac{1}{z}\right]=N$,

$$
\text { il existe } C^{\prime} \text { tel que, pour tout } m \in \mathbb{N}, z^{C^{\prime}} Q_{m} \subset M \bmod \pi^{q^{m}} \subset z^{-C^{\prime}} Q_{m} \text {. }
$$

b) Dans la situation de a) on note $P^{*}=\operatorname{Hom}_{\mathscr{Q}}(P, \mathscr{Q})$ et pour tout $m \in \mathbb{N}$ on note

$$
Q_{m}^{*}=\operatorname{Hom}_{\vartheta \widehat{\otimes} \vartheta_{L} / \pi^{q^{m}}}\left(Q_{m}, \oslash \widehat{\otimes} \vartheta_{L} / \pi^{q^{m}}\right) .
$$

Alors $P^{*}$ et le système des $Q_{m}^{*}$ vérifient les conditions $d u$ a) et on peut leur associer $N^{\prime}$ comme $N$ dans a). De plus ${ }^{\tau} P$ et $\left({ }^{\tau} Q_{m}\right)_{m \in \mathbb{N}}$ satisfont les conditions de a) et on peut leur associer $N^{\prime \prime}$ comme $N$ dans a). Alors on a des identifications naturelles $N^{\prime}=\operatorname{Hom}_{\oslash \widehat{\otimes} \vartheta_{L}\left[\frac{1}{z}\right]}\left(N, \oslash \widehat{\otimes} \vartheta_{L}\left[\frac{1}{z}\right]\right)$ et $N^{\prime \prime}={ }^{\tau} N$.

Remarque. - Dans a) l'hypothèse que $Q_{m}$ est libre est bien sûr nécessaire car si on prenait $P=\mathscr{Q}$ et $Q_{m}$ engendré par $1, \frac{\pi}{z}, \frac{\pi^{2}}{z^{2}}, \ldots, \frac{\pi^{q^{m}-1}}{z^{q^{m}-1}}$ la conclusion de a) serait fausse.

Démonstration de a). - Pour tout $n \geq m \geq 0$ on note

$$
Q_{m}^{n}=\sum_{i \geq n} z^{C} Q_{i} \bmod \pi^{q^{m}} .
$$

Alors $\left(Q_{m}^{n}\right)_{n \geq m}$ est une suite décroissante de sous- $\Theta \widehat{\otimes} \theta_{L} / \pi^{q^{m}}$-modules de $Q_{m}$ contenant $z^{2 C} Q_{m}$. Elle est stationnaire, $\operatorname{car}\left(Q_{m}^{n} / z^{2 C} Q_{m}\right)_{n \geq m}$ est une suite décroissante de sous- $k$-espaces vectoriels du $k$-espace vectoriel de dimension finie $Q_{m} / z^{2 C} Q_{m}$. On note $\widetilde{Q}_{m}$ la limite de la suite stationnaire $\left(Q_{m}^{n}\right)_{n \geq m}$. Donc $\widetilde{Q}_{m}$ est un sous- $\Theta \widehat{\otimes} \widehat{\vartheta}_{L} / \pi^{q^{m}}$-module de $Q_{m}$, on a $z^{2 C} Q_{m} \subset \widetilde{Q}_{m} \subset Q_{m}$, et pour $n \geq m$ on a une surjection $\widetilde{Q}_{n} \rightarrow \widetilde{Q}_{m}$. Soit $\left(x_{1}^{0}, \ldots, x_{r}^{0}\right)$ une base du $\Theta \widehat{\otimes} \theta_{L} / \pi$-module libre $z^{2 C} Q_{0}$ (qui est inclus dans $\widetilde{Q}_{0}$ ). Par récurrence sur $n$ on choisit $x_{1}^{n} \in \widetilde{Q}_{n}, \ldots, x_{r}^{n} \in \widetilde{Q}_{n}$ des relèvements de $x_{1}^{n-1}, \ldots, x_{r}^{n-1}$ de $\widetilde{Q}_{n-1}$ à $\widetilde{Q}_{n}$. Pour tout $i=1, \ldots, r$ la limite projective des $x_{i}^{n}$ définit un élément $x_{i} \in P$. On a même $x_{i} \in N$.

Soient $P^{*}$ et $\left(Q_{m}^{*}\right)_{m \in \mathbb{N}}$ comme dans l'énoncé de b). Comme $P^{*}$ et le système des $Q_{m}^{*}$ vérifient les conditions du a) on peut leur associer par la construction précédente $r$ éléments $\xi_{1}, \ldots, \xi_{r} \in P^{*}$ tels que pour $i \in\{1, \ldots, r\}$ et $n \in \mathbb{N}$ la réduction $\xi_{i}^{n}$ de $\xi_{i}$ modulo $\pi^{q^{n}}$ appartienne à $Q_{n}^{*}$ et que $\xi_{1}^{0}, \ldots, \xi_{r}^{0}$ forment une base de $z^{2 C} Q_{0}^{*}$. Pour $n \in \mathbb{N}$ et $i, j \in\{1, \ldots, r\}$, on a $\xi_{i}^{n} \in Q_{n}^{*}$ et $x_{j}^{n} \in Q_{n}$, d'où $\left\langle\xi_{i}, x_{j}\right\rangle \in \Theta \widehat{\otimes} \vartheta_{L} / \pi^{q^{m}}$. En passant à la limite on obtient $\left\langle\xi_{i}, x_{j}\right\rangle \in \Theta \widehat{\otimes} \vartheta_{L}$. Donc $a=\operatorname{det}\left(\left\langle\xi_{i}, x_{j}\right\rangle\right)$ est un élément de $\theta \widehat{\otimes} \vartheta_{L}$ dont la 
réduction modulo $\pi_{L}$ est non nulle (et appartient même à $\left.z^{4 C r}(\theta \widehat{\otimes} k)^{\times}\right)$. Pour tout $x \in N$ on a $\left\langle\xi_{i}, x\right\rangle \in \theta \widehat{\otimes} \theta_{L}\left[\frac{1}{z}\right]$. Donc on a un diagramme commutatif

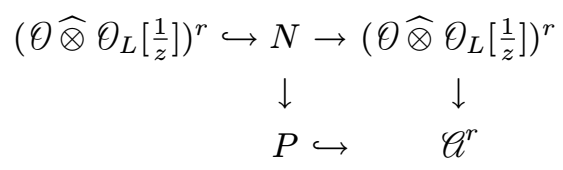

où les applications horizontales sont données par $\left(\alpha_{1}, \ldots, \alpha_{r}\right) \mapsto \alpha_{1} x_{1}+\cdots+\alpha_{r} x_{r}$ et $x \mapsto\left(\left\langle\xi_{1}, x\right\rangle, \ldots,\left\langle\xi_{r}, x\right\rangle\right)$ et où les flèches verticales sont les inclusions évidentes. Il résulte du diagramme que l'application $N \rightarrow\left(\theta \widehat{\otimes} \oslash_{L}\left[\frac{1}{z}\right]\right)^{r}$ est aussi une injection. Donc $N$ est un $\theta \widehat{\otimes} \theta_{L}\left[\frac{1}{z}\right]$-module de type fini sans torsion et il est libre grâce au lemme suivant et au théorème de structure des modules de type fini sur les anneaux principaux.

Lemme 8.12. - L'anneau $\theta \widehat{\otimes} \theta_{L}\left[\frac{1}{z}\right]$ est principal.

Démonstration. - L'anneau $\theta \widehat{\otimes} \vartheta_{L}$ est intègre, noethérien et intégralement clos dans son corps des fractions; il en donc de même de $\theta \widehat{\otimes} \Theta_{L}\left[\frac{1}{z}\right]$. Les idéaux premiers non nuls de $\theta \widehat{\otimes} \vartheta_{L}$ sont

- les idéaux principaux $(f)$, où $f$ est un élément irréductible de $\theta \widehat{\otimes} \theta_{L}$,

- l'idéal maximal $\left(z, \pi_{L}\right)$.

Par conséquent les idéaux premiers non nuls de $\Theta \widehat{\otimes} \vartheta_{L}\left[\frac{1}{z}\right]$ sont maximaux. Il en résulte déjà que $\theta \widehat{\otimes} \vartheta_{L}\left[\frac{1}{z}\right]$ est un anneau de Dedekind (voir par exemple [53], chapitre 1, proposition 4). De plus les idéaux premiers de $\theta \widehat{\otimes} \vartheta_{L}\left[\frac{1}{z}\right]$ sont principaux, comme on vient de le voir. Donc $\vartheta \widehat{\otimes} \theta_{L}\left[\frac{1}{z}\right]$ est principal (ibidem, chapitre 1, proposition 7).

Suite de la démonstration de a) du lemme 8.11. - Il reste à montrer (65). Notons $M_{0}$ le $\oslash \widehat{\otimes} \theta_{L}$-module libre engendré par $x_{1}, \ldots, x_{r}$. Soit $\widetilde{Q}$ la limite projective des $\widetilde{Q}_{n}$, c'est-à-dire

$$
\widetilde{Q}=\left\{x \in P, \forall m \in \mathbb{N}, x \bmod \pi^{q^{m}} \in \widetilde{Q}_{m}\right\} .
$$

Alors $\widetilde{Q}$ est un sous- $\Theta \widehat{\otimes} \vartheta_{L}$-module de $P$ qui contient $x_{1}, \ldots, x_{r}$. Comme $\widetilde{Q}_{m} \subset Q_{m}$ et $\xi_{i}^{m} \in Q_{m}^{*}$ pour tout $m \in \mathbb{N}$, on a $\left\langle\xi_{i}, x\right\rangle \in \widetilde{Q} \widehat{\otimes} \theta_{L}$ pour $i \in\{1, \ldots, r\}$ et $x \in \widetilde{Q}$, d'où $\widetilde{Q} \subset a^{-1} M_{0}$. Comme $\theta \widehat{\otimes} \vartheta_{L}$ est noethérien, $\widetilde{Q}$ est donc un $\theta \widehat{\otimes} \vartheta_{L}$-module de type fini. On a bien sûr

$$
\begin{gathered}
\left\{x \in P, \forall m \in \mathbb{N}, x \bmod \pi^{q^{m}} \in z^{2 C} Q_{m}\right\} \subset \widetilde{Q} \\
\subset\left\{x \in P, \forall m \in \mathbb{N}, x \bmod \pi^{q^{m}} \in Q_{m}\right\},
\end{gathered}
$$

donc $\widetilde{Q}$ engendre le $\theta \widehat{\otimes} \vartheta_{L}\left[\frac{1}{z}\right]$-module $N$. Soit $M$ un $\theta \widehat{\otimes} \vartheta_{L}$-module libre tel que $M \otimes{ }_{\vartheta \widehat{\otimes} \vartheta_{L}}$ $\theta \widehat{\otimes} \theta_{L}\left[\frac{1}{z}\right]=N$. Comme $M$ et $\widetilde{Q}$ sont des $\theta \widehat{\otimes} \theta_{L}$-modules de type fini qui engendrent $N$, il existe $C^{\prime}$ tel que $z^{C^{\prime}} M \subset \widetilde{Q} \subset z^{-C^{\prime}} M$. Pour tout $m \in \mathbb{N}$ on a $z^{2 C} Q_{m} \subset \widetilde{Q} \bmod \pi^{q^{m}}=$ $\widetilde{Q}_{m} \subset Q_{m}$ et donc $z^{2 C+C^{\prime}} Q_{m} \subset M \bmod \pi^{q^{m}} \subset z^{-C^{\prime}} Q_{m}$.

Démonstration du b) du lemme 8.11. - On utilise simplement (65). 
Fin de la démonstration de «i) implique ii) » dans la proposition 8.3

Grâce au lemme 8.11 on sait que $N$ est un $\theta \widehat{\otimes} \theta_{L}\left[\frac{1}{z}\right]$-module libre.

Montrons que $\phi_{D}^{-1}: D \otimes_{k((z))} \mathscr{G} \rightarrow{ }^{\tau} D \otimes_{k((z))} \mathscr{G}$ envoie $N$ dans ${ }^{\tau} N$ et que son image contient $(z-\pi)^{-s \tau} N$ où $s \in \mathbb{Z}_{-}$est tel que $V_{D} \supset(z-\pi)^{-s} U_{D}$. Pour tout $n \in \mathbb{N}$ on a

$$
(z-\pi)^{-s} \phi_{D}\left({ }^{\tau} \beta_{n}(\Delta)\right) \subset \beta_{n+1}(\Delta) \subset \phi_{D}\left({ }^{\tau} \beta_{n}(\Delta)\right) .
$$

La même relation a lieu modulo $\pi^{q^{n}}$ et grâce à b) du lemme 8.11 on en déduit

$$
(z-\pi)^{-s} \phi_{D}\left({ }^{\tau} N\right) \subset N \subset \phi_{D}\left({ }^{\tau} N\right) .
$$

Donc $\phi_{N}^{-1}: N \rightarrow{ }^{\tau} N$ est bien défini comme la restriction de $\phi_{D}^{-1}$ à $N$, et $\phi_{N}^{-1}(N) \supset(z-\pi)^{-s \tau} N$. On montre maintenant que $\left(N, \phi_{N}\right)$ satisfait la condition (PIL). On a

$$
\begin{aligned}
\phi_{D}{ }^{\tau} \phi_{D} \ldots \tau^{n-1} \phi_{D}\left(\tau^{n} \beta_{m}(\Delta)\right) & \subset\left((z-\pi) \cdots\left(z-\pi^{q^{n-1}}\right)\right)^{s} \beta_{m+n}(\Delta) \\
\text { et } \beta_{m+n}(\Delta) & \subset \phi_{D}^{\tau} \phi_{D} \ldots \tau^{n-1} \phi_{D}\left(\tau^{n} \beta_{m}(\Delta)\right) .
\end{aligned}
$$

Soit $M$ un $\oslash \widehat{\otimes} \vartheta_{L}$-module libre muni d'un isomorphisme $M\left[\frac{1}{z}\right]=N$. D'après (65) il existe $C^{\prime} \in \mathbb{N}$ tel que pour tout $l, m \in \mathbb{N}$, et en identifiant $M$ à $R(M)$,

$$
z^{C^{\prime}} M \bmod \pi^{q^{m}} \subset \beta_{m+l}(\Delta) \bmod \pi^{q^{m}} \subset z^{-C^{\prime}} M \bmod \pi^{q^{m}} .
$$

En appliquant (68) à $l=0$ et à $l=n$ on déduit de (66) que, pour $m, n \in \mathbb{N}$,

$$
z^{2 C^{\prime}}\left((z-\pi) \cdots\left(z-\pi^{q^{n-1}}\right)\right)^{-s} \phi_{N}{ }^{\tau} \phi_{N} \ldots \tau^{n-1} \phi_{N}\left(\tau^{n} M\right) \bmod \pi^{q^{m}} \subset M \bmod \pi^{q^{m}},
$$

et donc pour tout $n \in \mathbb{N}$,

$$
z^{2 C^{\prime}}\left((z-\pi) \cdots\left(z-\pi^{q^{n-1}}\right)\right)^{-s} \phi_{N}{ }^{\tau} \phi_{N} \cdots \tau^{n-1} \phi_{N}\left(\tau^{n} M\right) \subset M .
$$

De la même façon on déduit de (67) que, pour tout $m, n \in \mathbb{N}$,

$$
z^{2 C^{\prime}} M \bmod \pi^{q^{m}} \subset \phi_{N}{ }^{\tau} \phi_{N} \ldots \tau^{n-1} \phi_{N}\left(\tau^{n} M\right) \bmod \pi^{q^{m}}
$$

et donc pour tout $n \in \mathbb{N}$,

$$
z^{2 C^{\prime}} M \subset \phi_{N}{ }^{\tau} \phi_{N} \ldots \tau^{n-1} \phi_{N}\left(\tau^{n} M\right) .
$$

Grâce à (69) et (70), la condition (PIL) est satisfaite et donc $\left(N, \phi_{N}\right)$ est un isochtouca local.

Montrons maintenant que $\left(D, \phi_{D}, V_{D}\right)$ est associé à $N$. D'abord $\left(D, \phi_{D}\right)$ est la réduction de $\left(N, \phi_{N}\right)$ modulo $\pi_{L}$. Le raisonnement établissant l'unicité de la matrice $R$ dans le lemme 7.4 montre que l'inclusion de $N$ dans $D \otimes_{k((z))} \mathscr{C}$ est $R$.

Il reste à démontrer que $V_{D}$ est bien associé à $N$, c'est-à-dire que $V_{D}=\phi_{D}^{-1}\left(R\left(N \otimes_{\partial \otimes \vartheta_{L}\left[\frac{1}{z}\right]} L[[z-\pi]]\right)\right)$. Soit $M$ un $\oslash \widehat{\otimes} \vartheta_{L}$-module libre muni d'un isomorphisme $M\left[\frac{1}{z}\right]=N$. Alors (65) et (60) impliquent l'existence de $C$ tel que, pour tout $m \in \mathbb{N}$,

$$
z^{C} \beta_{m}(\Delta)\left[\left[\frac{\pi^{q^{m}}}{z}\right]\right] \subset M\left[\left[\frac{\pi^{q^{m}}}{z}\right]\right] \subset z^{-C} \beta_{m}(\Delta)\left[\left[\frac{\pi^{q^{m}}}{z}\right]\right]
$$

et donc

$$
N \otimes_{\partial \widehat{\otimes} \vartheta_{L}\left[\frac{1}{z}\right]} \Theta \widehat{\otimes} \vartheta_{L}\left[\left[\frac{\pi^{q^{m}}}{z}\right]\right]\left[\frac{1}{z}\right]=\beta_{m}(\Delta) \otimes_{\vartheta \widehat{\otimes} \vartheta_{L}} \oslash \widehat{\otimes} \vartheta_{L}\left[\left[\frac{\pi^{q^{m}}}{z}\right]\right]\left[\frac{1}{z}\right] .
$$

Pour tout $m>0$ on a une inclusion $\theta \widehat{\otimes} \vartheta_{L}\left[\left[\frac{\pi^{q^{m}}}{z}\right]\right]\left[\frac{1}{z}\right] \subset L[[z-\pi]]$. Comme $\phi_{D}^{-1}\left(\beta_{m}(\Delta)\right) \subset V_{D}$, on déduit de (71) appliqué à n'importe quel $m>0$ que $V \subset V_{D}$ 
où $V=\phi_{D}^{-1}\left(N \otimes_{\vartheta_{\widehat{Q}} \vartheta_{L}\left[\frac{1}{z}\right]} L[[z-\pi]]\right)$ est la structure de Hodge-Pink associée à $\left(N, \phi_{N}\right)$ (puisque $N$ désigne ici $R(N) \subset D \otimes_{k((z))} \mathscr{Q}$ ). Par la proposition 7.5 on a $\operatorname{det}(V)=(z-\pi)^{t_{N}(D)} \operatorname{det}(D) \otimes L[[z-\pi]]$ et on a vu au début de la preuve de « i) implique ii)» que $t_{N}(D)=t_{H}(D)$. Donc $\operatorname{det}(V)=\operatorname{det}\left(V_{D}\right)$ et $V=V_{D}$. Ceci termine la démonstration de la proposition 8.3.

Démonstration de la proposition 8.5. - Grâce à l'hypothèse que $k$ est algébriquement clos, on suppose $\left(D, \phi_{D}\right)$ défini sur $\mathbb{F}_{q}$ et décent au sens de [51] (les isochtoucas que nous considérons sont des « $\theta$-analogues » des $F$-isocristaux étudiés par Rapoport et Zink, la notion de décence est claire).

Si $\Delta$ et $\Delta^{\prime}$ sont deux réseaux de $D$, on note $l_{1}\left(\Delta, \Delta^{\prime}\right), \ldots, l_{r}\left(\Delta, \Delta^{\prime}\right)$ les diviseurs élémentaires de $\Delta^{\prime}$ par rapport à $\Delta$ : ce sont les uniques entiers relatifs tels que $l_{1}\left(\Delta, \Delta^{\prime}\right) \geq \cdots \geq l_{r}\left(\Delta, \Delta^{\prime}\right)$ et que pour une certaine base $e_{1}, \ldots, e_{r}$ de $\Delta$ sur $k[[z]]$, on ait $\Delta^{\prime}=z^{-l_{1}\left(\Delta, \Delta^{\prime}\right)} k[[z]] e_{1}+\cdots+z^{-l_{r}\left(\Delta, \Delta^{\prime}\right)} k[[z]] e_{r}$.

Lemme 8.13. - Soit $\left(D, \phi_{D}, V_{D}\right)$ un isochtouca de Hodge-Pink tel que $D=\underline{D} \otimes_{\mathbb{F}_{q}} k$ et $\phi_{D}=\phi_{\underline{D}} \otimes 1$ pour un certain isochtouca décent $\left(\underline{D}, \phi_{\underline{D}}\right)$ sur $\mathbb{F}_{q}$. Supposons $V_{D} \subset U_{D}$, $t_{H}(D)=t_{N}(D)$ et pour tout sous-isochtouca $D^{\prime}$ de dimension $1, t_{H}\left(D^{\prime}\right)<t_{N}\left(D^{\prime}\right)$. Soit $\underline{\Delta}$ un réseau de $\underline{D}$ et $\Delta=\underline{\Delta} \otimes_{\mathbb{F}_{q}} k$. Soit a $\in \mathbb{N}$. Il existe une constante $C_{0}$ telle que la propriété suivante soit vraie. Pour tout $n \in \mathbb{N}^{*}$, il existe une constante $C$ telle que pour tout réseau $\Delta^{\prime}$ de $D$ vérifiant

$$
\phi_{D}\left({ }^{\tau} \Delta^{\prime}\right) \subset z^{-a} \Delta^{\prime} \text { et } l_{1}\left(\Delta, \Delta^{\prime}\right) \geq l_{2}\left(\Delta, \Delta^{\prime}\right)+C,
$$

on ait $l_{1}\left(\Delta, \gamma_{n}\left(\Delta^{\prime}\right)\right) \leq l_{1}\left(\Delta, \Delta^{\prime}\right)-n+C_{0}$.

Démonstration. - D'après le lemme 2.18 de [51] et la proposition 1.6 de [52] (dont les démonstrations s'adaptent immédiatement aux $\theta$-analogues des $F$-isocristaux, que nous appelons ici isochtoucas), il existe des entiers $t, \tilde{a}, C_{1}$ (ne dépendant que de $D, \phi_{D}$ et $a$ ) tels que, pour tout réseau $\Delta^{\prime}$ de $D$ vérifiant $\phi_{D}\left({ }^{\tau} \Delta^{\prime}\right) \subset z^{-a} \Delta^{\prime}$, il existe un réseau $\Delta^{\prime \prime}$ de $D$ défini $\operatorname{sur} \mathbb{F}_{q^{t}}$, (c'est-à-dire provenant d'un réseau de $\underline{D} \otimes_{\mathbb{F}_{q}} \mathbb{F}_{q^{t}}$ ) vérifiant

$$
\phi_{D}\left({ }^{\tau} \Delta^{\prime \prime}\right) \subset z^{-\tilde{a}} \Delta^{\prime \prime} \text { et } \Delta^{\prime} \subset \Delta^{\prime \prime} \subset z^{-C_{1}} \Delta^{\prime} .
$$

En fait, en examinant les démonstrations de [51] et [52], on voit qu'on pourrait prendre $\tilde{a}=a$, mais on n'en a pas besoin ici. On a alors

$$
\begin{aligned}
l_{i}\left(\Delta, \Delta^{\prime}\right) & \leq l_{i}\left(\Delta, \Delta^{\prime \prime}\right) \leq l_{i}\left(\Delta, \Delta^{\prime}\right)+C_{1} \\
\text { et } \quad l_{i}\left(\Delta, \gamma_{n}\left(\Delta^{\prime}\right)\right) & \leq l_{i}\left(\Delta, \gamma_{n}\left(\Delta^{\prime \prime}\right)\right) \leq l_{i}\left(\Delta, \gamma_{n}\left(\Delta^{\prime}\right)\right)+C_{1} \text { pour tout } i
\end{aligned}
$$

Quitte à remplacer $C_{0}$ par $C_{0}+2 C_{1}, C$ par $C+2 C_{1}$ et $a$ par $\tilde{a}$, il suffit donc de montrer le lemme 8.13 en supposant de plus $\Delta^{\prime}$ défini sur $\mathbb{F}_{q^{t}}$. Le lemme suivant montre qu'avec cette condition supplémentaire l'énoncé du lemme 8.13 est vrai avec $C_{0}=0$. On est donc ramené à montrer le lemme suivant.

Lemme 8.14. - Soient $\left(D, \phi_{D}, V_{D}\right),\left(\underline{D}, \phi_{\underline{D}}\right), \underline{\Delta}$ et $\Delta$ comme dans le lemme précédent. Soient $a \in \mathbb{N}$ et $t, n \in \mathbb{N}^{*}$. On note $B$ l'ensemble des réseaux $\Delta^{\prime}$ de $D$ définis sur $\mathbb{F}_{q^{t}}$ et vérifiant $\phi_{D}\left({ }^{\tau} \Delta^{\prime}\right) \subset z^{-a} \Delta^{\prime}$. Il existe une constante $C$ telle que, pour tout $\Delta^{\prime} \in B$ vérifiant $l_{1}\left(\Delta, \Delta^{\prime}\right) \geq l_{2}\left(\Delta, \Delta^{\prime}\right)+C$, on ait $l_{1}\left(\Delta, \gamma_{n}\left(\Delta^{\prime}\right)\right) \leq l_{1}\left(\Delta, \Delta^{\prime}\right)-n$. 
Démonstration. - Si l'énoncé du lemme 8.14 est faux il existe une suite de réseaux $\Delta_{m}$ dans l'ensemble $B$ vérifiant $l_{1}\left(\Delta, \gamma_{n}\left(\Delta_{m}\right)\right)>l_{1}\left(\Delta, \Delta_{m}\right)-n$ et telle que $l_{1}\left(\Delta, \Delta_{m}\right)-l_{2}\left(\Delta, \Delta_{m}\right)$ tende vers l'infini. On note $\triangleq_{m}$ le $\mathbb{F}_{q^{t}}[[z]]$-réseau de $\underline{D} \otimes_{\mathbb{F}_{q}} \mathbb{F}_{q^{t}}$ tel que $\Delta_{m}=\triangleq_{m} \otimes_{\mathbb{F}_{q^{t}}} k$ (on souligne d'un trait les objets sur $\mathbb{F}_{q}$ et de deux traits les objets $\left.\operatorname{sur} \mathbb{F}_{q^{t}}\right)$. En notant $a_{m}=l_{1}\left(\Delta, \Delta_{m}\right)-l_{2}\left(\Delta, \Delta_{m}\right)$ on voit que $z^{l_{1}\left(\Delta, \Delta^{m}\right)} \triangleq_{m} / z^{a_{m}} \Delta \otimes_{\mathbb{F}_{q}} \mathbb{F}_{q^{t}}$ est un sous-module libre de rang 1 de $\Delta \otimes_{\left.\mathbb{F}_{q}[z]\right]}\left(\mathbb{F}_{q^{t}}[z] / z^{a_{m}}\right)$ et définit donc un point de $\mathbb{P}^{r-1}\left(\mathbb{F}_{q^{t}}[z] / z^{a_{m}}\right)$. Par compacité de $\mathbb{P}^{r-1}\left(\mathbb{F}_{q^{t}}[[z]]\right)$ muni de la topologie $z$-adique, on peut extraire une sous-suite ayant une limite et on note $\underline{\underline{D}}^{\prime}$ le sous- $\mathbb{F}_{q^{t}}((z))$-espace vectoriel de dimension 1 de $\underline{D} \otimes_{\mathbb{F}_{q}} \mathbb{F}_{q^{t}}$ tel que cette limite soit $\underline{\underline{D}}^{\prime} \cap\left(\underline{\Delta} \otimes_{\mathbb{F}_{q}} \mathbb{F}_{q^{t}}\right)$, sous- $\mathbb{F}_{q^{t}}[[z]]$-module libre de rang 1 de $\underline{\Delta} \otimes_{\mathbb{F}_{q}} \mathbb{F}_{q^{t}}$. On pose $D^{\prime}=\underline{\underline{D}}^{\prime} \otimes_{\mathbb{F}_{q^{t}}} k$ qui est un sous- $k((z))$-espace vectoriel de dimension 1 de $D$. Autrement dit, quitte à extraire une sous-suite il existe une suite d'entiers $b_{m}$ tendant vers $+\infty$ quand $m$ tend vers l'infini telle que

$$
z^{l_{1}\left(\Delta, \Delta_{m}\right)} \Delta_{m}+z^{b_{m}} \Delta=\left(D^{\prime} \cap \Delta\right)+z^{b_{m}} \Delta \text { pour tout } m .
$$

Comme les réseaux $\Delta_{m}$ appartiennent à $B$, en passant à la limite on voit que $D^{\prime}$ est un sousisochtouca de $D$ et on a déjà dit qu'il était défini sur $\mathbb{F}_{q^{t}}$. Le lemme 8.15 montre que, si $m$ est assez grand,

$$
l_{1}\left(\Delta, \gamma_{n}\left(\Delta_{m}\right)\right) \leq l_{1}\left(\Delta, \Delta_{m}\right)+n\left(t_{H}\left(D^{\prime}\right)-t_{N}\left(D^{\prime}\right)\right) .
$$

On obtient ainsi un sous-isochtouca $D^{\prime}$ de dimension 1 de $D$ tel que $t_{H}\left(D^{\prime}\right) \geq t_{N}\left(D^{\prime}\right)$, ce qui contredit l'hypothèse. Le lemme 8.14 est donc ramené au lemme suivant.

Lemme 8.15. - Soient $\left(D, \phi_{D}, V_{D}\right)$ un isochtouca de Hodge-Pink avec $V_{D} \subset U_{D}$ et $D^{\prime}=\left(D^{\prime}, \phi_{D^{\prime}}, V_{D^{\prime}}\right)$ un sous-isochtouca de rang 1 . Soit $\Delta \subset D$ un $k[[z]]-$ réseau. Soit $x$ un générateur de $D^{\prime}$. Pour tout $n \in \mathbb{N}$, il existe $C \in \mathbb{N}$ tel que pour $r \in \mathbb{N}$ assez grand,

$$
\gamma_{n}\left(k[[z]] x+z^{r} \Delta\right) \subset z^{n\left(t_{N}\left(D^{\prime}\right)-t_{H}\left(D^{\prime}\right)\right)} k[[z]] x+z^{r-C} \Delta .
$$

Démonstration. - On rappelle que $V_{D^{\prime}}=D^{\prime} \otimes_{k((z))} L((z-\pi)) \cap V_{D}$. On note $D^{\prime \prime}=D / D^{\prime}$ avec $\phi_{D^{\prime \prime}}$ induit par $\phi_{D}$ et $V_{D^{\prime \prime}}$ l'image de $V_{D}$ dans $D^{\prime \prime} \otimes_{k((z))} L((z-\pi))$, de sorte que $0 \rightarrow D^{\prime} \rightarrow D \rightarrow D^{\prime \prime} \rightarrow 0$ est une suite exacte courte dans la catégorie des isochtoucas de Hodge-Pink. On note $\beta_{n}^{D^{\prime}}, \beta_{n}^{D^{\prime \prime}}, \gamma_{n}^{D^{\prime}}$ et $\gamma_{n}^{D^{\prime \prime}}$ les applications correspondant à $\beta_{n}$ et $\gamma_{n}$ pour $D^{\prime}$ et $D^{\prime \prime}$. On note $\Delta^{\prime \prime}$ l'image de $\Delta$ dans $D^{\prime \prime}$. Soit $n \in \mathbb{N}$. Il existe $r_{0}$ tel que

$$
\beta_{n}\left(z^{-r_{0}} k[[z]] x+\Delta\right) \rightarrow \beta_{n}^{D^{\prime \prime}}\left(\Delta^{\prime \prime}\right)
$$

soit surjectif : on relève les vecteurs d'une base du $\theta \widehat{\otimes} \theta_{L}$-module libre

$$
\begin{gathered}
\beta_{n}^{D^{\prime \prime}}\left(\Delta^{\prime \prime}\right)=\left(\left(\phi_{D^{\prime \prime}}{ }^{\tau} \phi_{D^{\prime \prime}} \ldots \tau^{n-1} \phi_{D^{\prime \prime}} \tau^{n} \Delta^{\prime \prime}\right) \otimes_{k[[z]]} \oslash \widehat{\otimes} \theta_{L}\right) \\
\cap\left(\phi_{D^{\prime \prime}} \ldots \tau^{n-1} \phi_{D^{\prime \prime}}{ }^{n-1} V_{D^{\prime \prime}} \oplus \cdots \oplus \phi_{D^{\prime \prime}} V_{D^{\prime \prime}}\right)
\end{gathered}
$$

en des vecteurs de $\left(\phi_{D}{ }^{\tau} \phi_{D} \ldots \tau^{n-1} \phi_{D} \tau^{n} \Delta\right) \otimes_{k[[z]]} \oslash \widehat{\otimes} \vartheta_{L}$ que l'on corrige par des vecteurs de $\left(\phi_{D^{\prime}}{ }^{\tau} \phi_{D^{\prime}} \ldots \tau^{n-1} \phi_{D^{\prime}} \tau^{n} D^{\prime}\right) \otimes_{k((z))} \oslash \widehat{\otimes} \theta_{L}\left[\frac{1}{z}\right]$ pour qu'ils appartiennent à $\phi_{D} \cdots \tau^{n-1} \phi_{D} \tau^{n-1} V_{D} \oplus \cdots \oplus \phi_{D} V_{D}$, ce qui est possible, puisque

$$
\begin{aligned}
\vartheta \widehat{\otimes} \vartheta_{L}\left[\frac{1}{z}\right] \rightarrow L[[z-\pi]] /(z-\pi)^{-s} L[[z-\pi]] \oplus \cdots & \\
& \oplus L\left[\left[z-\pi^{q^{n-1}}\right]\right] /\left(z-\pi^{q^{n-1}}\right)^{-s} L\left[\left[z-\pi^{q^{n-1}}\right]\right]
\end{aligned}
$$


est surjectif (où $s \in \mathbb{Z}_{-}$est tel que $(z-\pi)^{-s} U_{D} \subset V_{D} \subset U_{D}$ ). Soit $r \geq r_{0}$. Comme $\beta_{n}\left(z^{-r_{0}} k[[z]] x+\Delta\right) \rightarrow \beta_{n}^{D^{\prime \prime}}\left(\Delta^{\prime \prime}\right)$ est surjectif de noyau $\beta_{n}^{D^{\prime}}\left(z^{-r_{0}} k[[z]] x\right)$ et $\beta_{n}\left(z^{-r} k[[z]] x+\Delta\right) \rightarrow \beta_{n}^{D^{\prime \prime}}\left(\Delta^{\prime \prime}\right)$ est surjectif de noyau $\beta_{n}^{D^{\prime}}\left(z^{-r} k[[z]] x\right)$ on a

$$
\begin{aligned}
\beta_{n}\left(k[[z]] x+z^{r} \Delta\right) & =\beta_{n}^{D^{\prime}}(k[[z]] x)+z^{r-r_{0}} \beta_{n}\left(k[[z]] x+z^{r_{0}} \Delta\right), \\
\text { donc } \gamma_{n}\left(k[[z]] x+z^{r} \Delta\right) & =\gamma_{n}^{D^{\prime}}(k[[z]] x)+z^{r-r_{0}} \gamma_{n}\left(k[[z]] x+z^{r_{0}} \Delta\right) .
\end{aligned}
$$

Comme $\gamma_{n}^{D^{\prime}}(k[[z]] x)=z^{n\left(t_{N}\left(D^{\prime}\right)-t_{H}\left(D^{\prime}\right)\right)} k[[z]] x$, le lemme 8.15 est démontré, ce qui achève aussi la démonstration des lemmes 8.13 et 8.14.

Fin de la démonstration de la proposition 8.5. - On rappelle que $k$ est supposé algébriquement clos. Soit $D=\left(D, \phi_{D}, V_{D}\right)$ un isochtouca de Hodge-Pink qui est un objet irréductible de $C_{\text {iso-HP-fa }}$ et tel que $V_{D} \subset U_{D}$.

Soit $a$ la constante du b) du lemme 8.7. On a $\phi_{D}\left({ }^{\tau} \gamma_{m}(\Delta)\right) \subset z^{-a} \gamma_{m}(\Delta)$ pour tout $m \in \mathbb{N}$. Soit $i \in\{1, \ldots, r-1\}$. Le lemme 8.13, appliqué à $\Lambda^{i} D$ montre l'existence de $C_{0} \in \mathbb{N}$ tel que pour tout $n \in \mathbb{N}$ il existe $C_{1}$ tel que si $\Delta^{\prime}$ est un réseau de $D$ vérifiant

$$
\phi_{D}\left({ }^{\tau} \Delta^{\prime}\right) \subset z^{-a} \Delta^{\prime} \text { et } l_{i}\left(\Delta, \Delta^{\prime}\right) \geq l_{i+1}\left(\Delta, \Delta^{\prime}\right)+C_{1},
$$

alors $\left(l_{1}+\cdots+l_{i}\right)\left(\Delta, \gamma_{n}\left(\Delta^{\prime}\right)\right) \leq\left(l_{1}+\cdots+l_{i}\right)\left(\Delta, \Delta^{\prime}\right)-n+C_{0}$.

En notant $C_{2}$ la constante qui apparaît dans e) du lemme 8.7 on a donc, pour tout $m \in \mathbb{N}$ tel que $l_{i}\left(\Delta, \gamma_{m}(\Delta)\right) \geq l_{i+1}\left(\Delta, \gamma_{m}(\Delta)\right)+C_{1}$,

$$
\left(l_{1}+\cdots+l_{i}\right)\left(\Delta, \gamma_{n+m}(\Delta)\right) \leq\left(l_{1}+\cdots+l_{i}\right)\left(\Delta, \gamma_{m}(\Delta)\right)-n+C_{0}+i C_{2} .
$$

Comme $\operatorname{det}\left(\gamma_{m}(\Delta)\right)=\operatorname{det}(\Delta)$ pour tout $m \in \mathbb{N}$, on a $\left(l_{1}+\cdots+l_{r}\right)\left(\Delta, \gamma_{m}(\Delta)\right)=0$. On a $z^{a} \gamma_{m}(\Delta) \subset \gamma_{m+1}(\Delta) \subset z^{-a} \gamma_{m}(\Delta)$ pour tout $m \in \mathbb{N}$. En appliquant l'inégalité (72) avec $n=C_{0}+(r-1) C_{2}$ et $i \in\{1, \ldots, r-1\}$, et en appliquant le lemme suivant avec

$$
C=\max \left(C_{1}, n a\right) \text { et } l_{i}^{m}=l_{i}\left(\Delta, \gamma_{m n}(\Delta)\right) \text { pour } i \in\{1, \ldots, r\} \text { et } m \in \mathbb{N},
$$

on voit qu'il existe $C_{3}$ tel que pour tout $m \in \mathbb{N}, z^{C_{3}} \Delta \subset \gamma_{m n}(\Delta) \subset z^{-C_{3}} \Delta$. On a donc $z^{C_{3}+\left[\frac{m}{2}\right] a} \Delta \subset \gamma_{m}(\Delta) \subset z^{-C_{3}-\left[\frac{m}{2}\right] a} \Delta$ pour tout $m \in \mathbb{N}$. On est donc ramené à montrer le lemme suivant. Pour lui donner un sens géométrique, signalons que si $\Delta^{\prime}$ est un réseau, le polygone concave ayant pour sommets $\left(i,\left(l_{1}+\cdots+l_{i}\right)\left(\Delta, \Delta^{\prime}\right)\right)$ pour $i=0, \ldots, r$ peut être appelé « polygone des invariants» de $\Delta^{\prime}$ relativement à $\Delta$ et que les $l_{i}\left(\Delta, \Delta^{\prime}\right)$ sont les pentes de ce polygone. Dans le cas que l'on considère on a toujours $\left(l_{1}+\cdots+l_{r}\right)\left(\Delta, \Delta^{\prime}\right)=0$, c'est-à-dire que le premier sommet est $(0,0)$ et le dernier est $(r, 0)$.

Lemme 8.16. - Soient $r \in \mathbb{N}^{*}$ et $C \in \mathbb{R}_{+}$. Soient $l_{i}^{n} \in \mathbb{R}$ pour $i \in\{1, \ldots, r\}$ et $n \in \mathbb{N}$ vérifiant

i) pour tout $n \in \mathbb{N}, l_{1}^{n} \geq \cdots \geq l_{r}^{n}$,

ii) pour tout $n \in \mathbb{N}, \sum_{i=1}^{r} l_{i}^{n}=0$,

iii) on a $l_{1}^{0}=\cdots=l_{r}^{0}=0$,

iv) pour $i \in\{1, \ldots, r\}$ et $n \in \mathbb{N}, l_{i}^{n}-C \leq l_{i}^{n+1} \leq l_{i}^{n}+C$,

v) pour $i \in\{1, \ldots, r-1\}$ et $n \in \mathbb{N}$, si $l_{i}^{n}-l_{i+1}^{n} \geq C$, on $a$

$$
l_{1}^{n+1}+\cdots+l_{i}^{n+1} \leq l_{1}^{n}+\cdots+l_{i}^{n} .
$$

Alors on a $l_{i}^{n} \in\left[-C^{\prime}, C^{\prime}\right]$ pour tout $i \in\{1, \ldots, r\}$ et pour tout $n \in \mathbb{N}$, avec $C^{\prime}$ ne dépendant que de $r$ et de $C$. 
Démonstration. - Pour tout $n \in \mathbb{N}$ on note $P_{n}$ le polygone concave dont les sommets sont les $\left(i, P_{n}(i)\right)$ pour $i \in\{0, \ldots, r\}$, avec

$$
P_{n}(i)=l_{1}^{n}+\cdots+l_{i}^{n}
$$

(en particulier le premier sommet est $(0,0)$ et le dernier est $(r, 0)$ ). La différence des pentes au sommet $\left(i, P_{n}(i)\right)$, que nous appellerons brisure, est $l_{i}^{n}-l_{i+1}^{n}$. La condition v) assure que $P_{n+1}(i) \leq P_{n}(i)$ si la brisure de $P_{n}$ en $\left(i, P_{n}(i)\right)$ est $\geq C$. L'idée naïve de la démonstration est la suivante $:$ si $\max _{i} P_{n}(i)$ est atteint en un sommet $\left(j, P_{n}(j)\right)$ de brisure $\geq C$ la condition v) impose $P_{n+1}(j) \leq P_{n}(j)$ et si $\max _{i} P_{n+1}(i)$ est atteint pour la même valeur de $i$ (c'est-à-dire $i=j$ ) on en déduit $\max _{i} P_{n+1}(i) \leq \max _{i} P_{n}(i)$, ce qui impose à $P_{n}$ de rester borné. Nous allons voir qu'en remplaçant $P_{n}$ par $P_{n}-Q$ pour un certain $Q$ de brisure constante $3 C$ cet argument devient correct. On pose donc $Q(i)=\frac{3 C}{2} i(r-i)$ pour $i \in\{0, \ldots, r\}$. On pose ensuite $m_{n}=\max _{i \in\{0, \ldots, r\}}\left(P_{n}(i)-Q(i)\right) \in \mathbb{R}_{+}$. Nous allons montrer que $m_{n+1} \leq m_{n}$ pour tout $n \in \mathbb{N}$. Comme $m_{n} \geq 0$, il n'y a rien à démontrer si $m_{n+1}=0$. Supposons donc $m_{n+1}>0$ et soit $j \in\{1, \ldots, r-1\}$ tel que le maximum soit atteint en $j$. La brisure de $P_{n+1}-Q$ en $j$ est donc $\geq 0$. Or la brisure de $Q$ est égale à $3 C$, donc $l_{j}^{n+1}-l_{j+1}^{n+1} \geq 3 C$. Grâce à la condition iv) on en déduit $l_{j}^{n}-l_{j+1}^{n} \geq C$, d'où par la condition v), $P_{n}(j) \geq P_{n+1}(j)$. On a donc

$$
m_{n} \geq P_{n}(j)-Q(j) \geq P_{n+1}(j)-Q(j)=m_{n+1} .
$$

Par la condition iii) on a $m_{0}=0$. Donc $m_{n}=0$ pour tout $n \in \mathbb{N}$. Donc

$$
\begin{aligned}
l_{n}^{1} & =P_{n}(1) \leq Q(1)=\frac{3}{2} C(r-1) \\
\text { et } \quad l_{n}^{r} & =-P_{n}(r-1) \geq-Q(r-1)=-\frac{3}{2} C(r-1) \text { pour tout } n \in \mathbb{N} .
\end{aligned}
$$

Ceci termine la démonstration du lemme $8.16\left(\right.$ avec $C^{\prime}=\frac{3}{2} C(r-1)$ ) et donc celle de la proposition 8.5.

\section{Un contre-exemple à « faiblement admissible implique admissible » sur un anneau de valuation réelle non discrète}

Le contre-exemple que nous allons présenter est dû à Pink [50] dans le cadre de l'uniformisation des t-motifs. Nous l'avons adapté à la théorie de Fontaine, et Hartl l'a fait de façon indépendante (voir [29]). L'idée de ce contre-exemple vient des $\theta$-analogues des anneaux de Fontaine (voir [31, 32]), mais nous allons l'expliquer sans anneaux de Fontaine. Nous incluons ce contre-exemple dans notre texte parce que la démonstration du lemme 9.1 ci-dessous est voisine de celle de la proposition 7.5.

En général soit $\theta_{L}$ un anneau de valuation réelle non nécessairement discrète, complet, d'idéal maximal $m_{L}$, de corps résiduel $k$. On suppose $\theta_{L}$ muni d'une structure de $\theta$-algèbre, telle que l'image de $\pi$ soit dans $m_{L}$ et soit non nulle. Comme Hartl dans [31] on suppose donnée une section $k \rightarrow \theta_{L}$. Soit $\left(D, \phi_{D}\right)$ un isochtouca local sur $k$.

À tout isochtouca local $\left(N, \phi_{N}\right) \operatorname{sur} \theta_{L}$ rigidifié par un isomorphisme $\rho$ entre sa restriction à $\theta_{L} / \pi \theta_{L}$ et l'extension de $\left(D, \phi_{D}\right)$ à $\theta_{L} / \pi \theta_{L}$ par la section $k \rightarrow \theta_{L} \rightarrow \theta_{L} / \pi \theta_{L}$, on associe (comme dans le paragraphe 3) une structure de Hodge-Pink $V$, c'est-à-dire un $L[[z-\pi]]$-réseau dans $D \otimes_{k((z))} L((z-\pi))$. On dit qu'une structure de Hodge-Pink $V$ est 
faiblement admissible si pour tout sous-isochtouca local $D^{\prime}$ de $D$, muni de la structure de Hodge-Pink $V_{D^{\prime}}=V_{D} \cap D^{\prime} \otimes_{k((z))} L((z-\pi))$ on a $t_{H}\left(D^{\prime}\right) \leq t_{N}\left(D^{\prime}\right)$. Hartl montre dans [31] que toute structure de Hodge-Pink associée à un isochtouca local comme ci-dessus est faiblement admissible (on peut aussi le montrer en reprenant la démonstration de la proposition 7.5).

Supposons que $\theta_{L}$ possède un élément $a$ de valuation $>0$ ainsi que les $a^{q^{i}}$ pour $i \in \mathbb{Z}$ (cela implique que la valuation est non discrète mais elle peut être rationnelle). Nous allons construire un isochtouca local sur $k$ muni d'une structure de Hodge-Pink faiblement admissible, qui ne correspond à aucun isochtouca local sur $\theta_{L}$. Dans [31], Hartl a montré que faiblement admissible implique admissible est vrai sur tout anneau de valuation réelle complet de corps résiduel algébriquement clos qui ne possède pas un tel élément $a$. En fait il montre même que tout contre-exemple est obtenu comme celui qui va suivre.

Voici le contre-exemple. On note $\mathscr{C}=\mathscr{C}\left(\theta_{L}\right)$ (défini dans le paragraphe 3 ).

Lemme 9.1. - Soient $\left(N, \phi_{N}\right)$ un isochtouca local sur $\theta_{L}, k \in \mathbb{N}^{*}$ et $j, l \in \mathbb{Z}$ tels que $\frac{l}{k}+j>0$. Alors tout $x \in \alpha^{j} N \otimes_{\oslash \widehat{\otimes} \vartheta_{L}\left[\frac{1}{z}\right]} \mathscr{C}$ tel que $z^{l} \phi_{N} \ldots \tau^{k-1} \phi_{N}\left({ }^{k} x\right)=x$ est nul.

Dans ce lemme l'inégalité stricte $\frac{l}{k}+j>0$ est nécessaire : si $k=1, j=0$ et $l=0$ on peut prendre $N=\emptyset \widehat{\otimes} \theta_{L}\left[\frac{1}{z}\right], \phi_{N}=1$ et $x=1$.

Démonstration. - En remplaçant $j$ par $0, x$ par $\alpha^{-j} x, \phi_{N} \operatorname{par}(z-\pi)^{-j} \phi_{N}$ et $l$ par $l+k j$ on se ramène à montrer le lemme pour $j=0$, ce que l'on suppose désormais. On rappelle que pour $w \in \mathbb{R}_{+}$on possède la valuation $v_{w} \operatorname{sur} \mathscr{C}$, définie par

$$
v_{w}\left(\sum_{n \in \mathbb{Z}} a_{n} z^{n}\right)=\inf _{n \in \mathbb{Z}} n w+v\left(a_{n}\right)
$$

où $v$ est la valuation sur $\theta_{L}$, normalisée par $v(\pi)=1$. On rappelle que $v_{w}$ s'étend en une valuation sur $\mathscr{C}\left[\frac{1}{\alpha}\right]$. On choisit une base de $N$ sur $\Theta \widehat{\otimes} \vartheta_{L}\left[\frac{1}{z}\right]$. On définit la valuation $v_{w}$ d'un élément de $N \otimes \mathscr{C}$ comme le minimum des valuations $v_{w}$ de ses coordonnées. Par la condition (PIL) il existe $s \in \mathbb{Z}$ et $C \in \mathbb{N}$ tels que pour tout $n \in \mathbb{N}$ la matrice de $\phi_{N} \ldots \tau^{n-1} \phi_{N}$ appartienne à $z^{-C}(z-\pi)^{s} \cdots\left(z-\pi^{q^{n-1}}\right)^{s} M_{r}\left(\Theta \widehat{\otimes} \theta_{L}\right)$.

Soit $t \in \mathbb{N}$. On a $x=z^{t l} \phi_{N} \ldots \tau^{t k-1} \phi_{N}\left(\tau^{t k} x\right)$. Comme $v_{q^{t k}}$ est une valuation on en déduit

$$
v_{q^{t k}}(x) \geq t l q^{t k}+s\left(1+q+\cdots+q^{t k-1}\right)-C q^{t k}+q^{t k} v_{1}(x),
$$

et donc grâce à l'hypothèse $l>0$ il existe $\epsilon>0$ tel que $v_{q^{t k}}(x) \geq \epsilon t q^{t k}$ pour $t$ assez grand. Comme $x \in N \otimes_{\vartheta \widehat{\otimes} \vartheta_{L}\left[\frac{1}{z}\right]} \mathscr{C}$ cela implique $x=0$ car si $x=\sum_{n \in \mathbb{Z}} x_{n} z^{n}$, on a $v\left(x_{n}\right) \geq(\epsilon t-n) q^{t k}$ pour tout $t$ assez grand, donc $x_{n}=0$ pour tout $n \in \mathbb{Z}$.

Nous allons maintenant construire un isochtouca de Hodge-Pink $\left(D, \phi_{D}, V_{D}\right)$ faiblement admissible qui n'est associé à aucun isochtouca local. On pose $D=k((z))^{5}$, $\phi_{D}=\left(\begin{array}{lllll}0 & 0 & 0 & 0 & z^{2} \\ 1 & 0 & 0 & 0 & 0 \\ 0 & 1 & 0 & 0 & 0 \\ 0 & 0 & 1 & 0 & 0 \\ 0 & 0 & 0 & 1 & 0\end{array}\right)$. On choisira plus tard $V_{D}$ tel que $U_{D} \subset V_{D} \subset(z-\pi)^{-1} U_{D}$ et 
$\operatorname{dim}_{L} V_{D} / U_{D}=2$. Comme $\left(D, \phi_{D}\right)$ ne contient aucun sous-isochtouca autre que 0 et lui-même, $\left(D, \phi_{D}, V_{D}\right)$ est faiblement admissible.

$$
\text { Posons } x=\left(\begin{array}{c}
\sum_{n \in \mathbb{Z}} a^{q^{-10 n}} z^{n} \\
\sum_{n \in \mathbb{Z}} a^{q^{-10 n-4}} z^{n} \\
\sum_{n \in \mathbb{Z}} a^{q^{-10 n-8}} z^{n} \\
\sum_{n \in \mathbb{Z}} a^{q^{-10 n-12}} z^{n} \\
\sum_{n \in \mathbb{Z}} a^{q^{-10 n-16}} z^{n}
\end{array}\right) \in D \otimes_{k((z))} \text { C. On a } z^{-1} \phi_{D}{ }^{\tau} \phi_{D}\left(\tau^{2} x\right)=x \text {. }
$$

Choisissons $V_{D}$ tel que les images de $\phi_{D}^{-1}(x)$ et ${ }^{\tau} x$ dans ${ }^{\tau} D \otimes_{k((z))} L[[z-\pi]]$ appartiennent à $(z-\pi) V_{D}$. Grâce à l'équation $z^{-1} \phi_{D}{ }^{\tau} \phi_{D}\left({ }^{\tau^{2}} x\right)=x$, on en déduit que pour tout $n \in \mathbb{N}$ l'image de $x$ dans $D \otimes_{k((z))} L\left[\left[z-\pi^{q^{n}}\right]\right]$ appartient à $\left(z-\pi^{q^{n}}\right) \phi_{D} \cdots \tau^{n} \phi_{D}\left(\tau^{n} V_{D}\right)$.

Supposons que $\left(D, \phi_{D}, V_{D}\right)$ est associé à un isochtouca local $\left(N, \phi_{N}\right)$ sur $\theta_{L}$. Le début de la démonstration de la proposition 3.12 indique que

$$
\begin{gathered}
R\left(N \otimes_{\oslash \widehat{\otimes} \vartheta_{L}\left[\frac{1}{z}\right]} \mathscr{C}\right)=\left\{x \in D \otimes_{k((z))} \mathscr{C}\left[\frac{1}{\alpha}\right], \forall n \in \mathbb{N}, \text { l'image de } x\right. \text { dans } \\
\left.D \otimes_{k((z))} L\left[\left[z-\pi^{q^{n}}\right]\right] \text { appartient à } \phi_{D} \ldots \tau^{n} \phi_{D}\left(\tau^{n} V_{D}\right)\right\} .
\end{gathered}
$$

On en déduit que $R^{-1}(x)$ appartient à $\alpha N \otimes_{\widehat{\partial} \widehat{\otimes} \vartheta_{L}\left[\frac{1}{z}\right]} \mathscr{C}$. Comme $R^{-1}(x)$ vérifie $z^{-1} \phi_{N}{ }^{\tau} \phi_{N}\left(\tau^{2}\left(R^{-1}(x)\right)\right)=R^{-1}(x)$, le lemme 9.1 amène une contradiction (avec $k=2, j=1$ et $l=-1)$.

Remarque. - On peut montrer que les images de $\phi_{D}^{-1}(x)$ et ${ }^{\tau} x$ dans ${ }^{\tau} D \otimes_{k((z))}(L[[z-\pi]] /(z-\pi) L[[z-\pi]])$ sont indépendantes, ce qui fait que $V_{D}$ est déterminé par $a$. En effet $y=x \wedge \phi_{D}\left({ }^{\tau} x\right) \in \Lambda^{2} D \otimes_{k((z))} \mathscr{C}$ est non nul et vérifie

$$
z^{-1}\left(\Lambda^{2}\left(\phi_{D}\right)\right)\left({ }^{\tau} y\right)=-y \text {. }
$$

Or si $x$ et $\phi_{D}\left({ }^{\tau} x\right)$ sont égaux en $z=\pi, y$ s'annule en $z=\pi$ donc aussi, grâce à (73), en tous les $z=\pi^{q^{n}}$ pour $n \in \mathbb{N}$, et on a donc $y \in \alpha \Lambda^{2} D \otimes_{k((z))} \mathscr{C}$. Or ceci et l'équation $z^{-1 \tau^{5}} y=-y$ qui résulte de (73) contredisent le lemme 9.1, appliqué à $N=\left(\Theta \widehat{\otimes} \theta_{L}\left[\frac{1}{z}\right]\right)^{10}, \phi_{N}=-$ Id, $k=5, l=-1$ et $j=1$.

\section{Théorie entière pour les chtoucas locaux minuscules sur un anneau de valuation discrète}

Dans tout ce paragraphe on impose $q \geq 3$. Soit $\theta_{L}$ comme dans le paragraphe $7: \theta_{L}$ un anneau de valuation discrète complet de corps résiduel $k, m_{L}$ son idéal maximal, $\pi_{L}$ une uniformisante de $\theta_{L}, L$ son corps des fractions et on suppose $\theta_{L}$ muni d'une structure de $\theta$-algèbre de sorte que l'image de $\pi$ dans $\theta_{L}$ soit un élément non nul de $m_{L}$. On note $e$ l'indice de ramification de $L$ sur $K$.

On note $C_{\min }$ la catégorie des chtoucas locaux minuscules sur $\theta_{L}$. C'est une catégorie Ө-linéaire, mais non abélienne. On rappelle que ses objets sont les $\theta \widehat{\otimes} \theta_{L}$-modules libres $M$ munis de

$$
\phi_{M} \in \operatorname{Hom}_{\partial \widehat{\partial} \theta_{L}}\left({ }^{\tau} M, M\right) \text { et } \psi_{M} \in \operatorname{Hom}_{\partial \widehat{\partial} \theta_{L}}\left(M,{ }^{\tau} M\right)
$$

tels que $\phi_{M} \circ \psi_{M}=(z-\pi)$ et $\psi_{M} \circ \phi_{M}=(z-\pi)$.

D'après le paragraphe 5 on a un foncteur $\mathbb{D}$ de la catégorie des chtoucas locaux minuscules $\operatorname{sur} \theta_{L} / \pi \theta_{L}$ vers la catégorie des $\theta$-cristaux (au sens de Honda et Gross-Hopkins) sur 
$\theta_{L} / \pi \theta_{L}$. A fortiori on obtient un foncteur de la catégorie des chtoucas locaux minuscules sur $\theta_{L} / \pi \theta_{L}$ vers la catégorie des $\theta$-cristaux relativement à $\left(\theta, \pi \theta, \gamma_{\vartheta}\right)$ sur $\theta_{L} / \pi \theta_{L}$, que l'on note encore $\mathbb{D}$. Grâce au lemme 1.2 tout chtouca local minuscule sur $\theta_{L} / \pi \theta_{L}$ se relève en un chtouca local minuscule sur $\theta_{L}$. En utilisant ce fait, nous allons donner une description plus simple du foncteur $\mathbb{D}$, qui servira dans la preuve du théorème 10.3.

Soit $B=(\Theta \widehat{\otimes} k)[[u]]$, muni de la topologie $(\pi, u)$-adique. On note $I_{0}$ le noyau du morphisme surjectif de $\theta$-algèbres $B \rightarrow \theta_{L}$ qui à $u$ associe $\pi_{L}$, de sorte que l'on a un isomorphisme $s: \theta_{L} \rightarrow B / I_{0}$. On pose $I=I_{0}+\pi B$ et on voit facilement que $I=\left(\pi, u^{e}\right)$. On pose ensuite $\hat{B}=(\Theta \widehat{\otimes} k)\left[\left[u, \frac{u^{e q}}{\pi}\right]\right]$, muni de la topologie $\left(\pi, \frac{u^{e q}}{\pi}\right)$-adique (c'est-à-dire le quotient de $\theta \widehat{\otimes} k[[u, v]]$ muni de la topologie $(\pi, u, v)$-adique par l'idéal fermé engendré par $\pi v-u^{e q}$ ). On note $\beta: B \rightarrow \hat{B}$ l'inclusion naturelle (qui est $\theta$-linéaire et continue). Enfin on définit $\gamma: I \rightarrow \hat{B}$ en posant $\gamma\left(u^{e}\right)=\frac{u^{e q}}{\pi}$ et $\gamma(\pi)=\pi^{q-1}$. Il est clair que $(\hat{B}, \beta, \gamma)$ est une structure de puissances divisées sur $I$ compatible à $\left(\theta, \pi \theta, \gamma_{\vartheta}\right)$.

L'isomorphisme $s: \theta_{L} \rightarrow B / I_{0}$ considéré précédemment induit un isomorphisme $s: \theta_{L} / \pi \theta_{L} \rightarrow B / I$. Soit $\hat{J}$ l'idéal de $\hat{B}$ engendré par $\pi$ et $\frac{u^{e q}}{\pi}$. Pour tout $n$, $\left(B / I^{n}, I / I^{n}, s, \hat{B} / \hat{J}^{n}, \beta, \gamma\right)$ est un $\theta_{L} / \pi \theta_{L}$-objet test relativement à $\left(\theta, \pi \Theta, \gamma_{\vartheta}\right)$ au sens du paragraphe 5 (où $I^{n}$ désigne l'idéal engendré par les produits de $n$ éléments de $I$ et de même pour $\hat{J})$.

Soient $\left(M, \phi_{M}\right) \in C_{\min }, r$ le rang de $M$ sur $\theta \widehat{\otimes} \theta_{L}$ et $\mathbb{D}\left(M, \phi_{M}\right)$ le $\theta$-cristal relativement à $\left(\theta, \pi \theta, \gamma_{\vartheta}\right)$ sur $\theta_{L} / \pi \theta_{L}$ associé à la réduction de $\left(M, \phi_{M}\right)$ modulo $\pi$ (la notation $\mathbb{D}\left(M, \phi_{M}\right)$ est donc un léger abus). Alors la limite projective des $\hat{B} / \hat{J}^{n}$-modules libres $\left(\mathbb{D}\left(M, \phi_{M}\right)\right)\left(B / I^{n}, I / I^{n}, s, \hat{B} / \hat{J}^{n}, \beta, \gamma\right)$ est un $\hat{B}$-module libre de rang $r$ que l'on note $\left(\mathbb{D}\left(M, \phi_{M}\right)\right)(B, I, s, \hat{B}, \beta, \gamma)$ par abus et qui ne dépend donc que de la réduction de $\left(M, \phi_{M}\right)$ modulo $\pi$.

Pour le déterminer on relève le chtouca local $\left(M, \phi_{M}\right)$ de $\theta_{L}$ à $B$ en un objet $\left(\widetilde{M}, \widetilde{\phi_{M}}\right)$ qui n'est plus nécessairement un chtouca local, c'est-à-dire que $\widetilde{M}$ est un $\theta \widehat{\otimes} B$-module libre et $\widetilde{\phi_{M}}:{ }^{\tau} \widetilde{M} \rightarrow \widetilde{M}$ est $\theta \widehat{\otimes} B$-linéaire, mais son image ne contient pas nécessairement $(z-\pi) \widetilde{M}$. Pour faire cela on construit un relèvement $\kappa: \theta_{L} \rightarrow B$ de $s: \theta_{L} \rightarrow B / I_{0}$ en envoyant $\pi_{L}$ sur $u$ (ce relèvement n'est pas $\theta$-linéaire et n'a rien de canonique). On pose alors $\widetilde{M}=M \otimes_{\vartheta \widehat{\otimes} \vartheta_{L}, 1 \widehat{\otimes} \kappa} \oslash \widehat{\otimes} B$, muni de $\widetilde{\phi_{M}}=\phi_{M} \widehat{\otimes} 1$. L'image de $\widetilde{\phi_{M}}$ ne contient pas nécessairement $(z-\pi) \widetilde{M}$ parce que $\kappa$ n'est pas $\theta$-linéaire. Alors grâce à la proposition 5.14, $\left(\mathbb{D}\left(M, \phi_{M}\right)\right)(B, I, s, \hat{B}, \beta, \gamma)$ est

$$
\left({ }^{\tau} \widetilde{M} /(z-\pi) \tau \widetilde{M}\right) \otimes_{B} \hat{B}={ }^{\tau} M \otimes_{\vartheta \widehat{\otimes} \vartheta_{L}} \hat{B},
$$

où l'inclusion $\oslash \widehat{\otimes} \vartheta_{L} \subset \hat{B}$ est la composée $\theta \widehat{\otimes} \vartheta_{L} \stackrel{1 \widehat{\otimes} \kappa}{\rightarrow} \oslash \widehat{\otimes} B \stackrel{z \mapsto \pi}{\rightarrow} B \stackrel{\beta}{\rightarrow} \hat{B}$. La structure de $\theta$-algèbre sur $\hat{B}$ qui envoie $\pi$ sur $\pi$ est canonique mais cette inclusion $\theta \widehat{\otimes} \theta_{L} \subset \hat{B}$ qui envoie $z$ sur $\pi$ et $\pi_{L}$ sur $u$ n'est pas canonique.

On a donc une inclusion $\theta \widehat{\otimes} \vartheta_{L} \subset \hat{B}$ telle que

$$
\left(\mathbb{D}\left(M, \phi_{M}\right)\right)(B, I, s, \hat{B}, \beta, \gamma)={ }^{\tau} M \otimes_{\emptyset \widehat{\otimes} \theta_{L}} \hat{B} .
$$

Ceci permet une approche naïve, où l'on retrouve heuristiquement l'anneau $\hat{B}$ en évitant le paragraphe 5 . Nous verrons dans la proposition 10.2 ci-dessous que cette approche fournit 
une construction plus simple du foncteur $\mathbb{D}$ de la catégorie des chtoucas locaux minuscules sur $\theta_{L} / \pi \theta_{L}$ vers la catégorie des $\theta$-cristaux relativement à $\left(\theta, \pi \theta, \gamma_{\theta}\right)$ sur $\theta_{L} / \pi \theta_{L}$.

On cherche un anneau $\&$ (noté ainsi par Breuil dans [14] et noté $R_{V}$ dans [20]) contenant $\oslash \widehat{\otimes} \theta_{L}$ tel que le $\&$-module ${ }^{\tau} M \otimes_{\partial \widehat{\otimes} \vartheta_{L}} \&$ ne dépende que de la réduction de $\left(M, \phi_{M}\right)$ modulo $\pi$.

Commençons par un exemple (dans les notations de la démonstration du lemme 7.4).

Soient $r=2, M=M^{\prime}=\left(\Theta \widehat{\otimes} \theta_{L}\right)^{2}, \phi_{M}=\left(\begin{array}{rr}z-\pi & 0 \\ 0 & 1\end{array}\right)$ et $\phi_{M^{\prime}}=\left(\begin{array}{rr}z-\pi & 0 \\ \pi & 1\end{array}\right)$.

Un calcul facile montre que $R^{\prime-1} R$, qui est égal à la limite quand $n$ tend vers l'infini de $\phi_{M^{\prime}}{ }^{\tau} \phi_{M^{\prime}} \ldots \tau^{n} \phi_{M^{\prime}} \tau^{n} \phi_{M}^{-1} \ldots{ }^{\tau} \phi_{M}^{-1} \phi_{M}^{-1}$, vaut

$$
\left(\begin{array}{c}
1 \\
0 \\
\frac{\pi}{z}\left(1-\frac{\pi}{z}\right)^{-1}+\frac{\pi^{q}}{z^{2}}\left(1-\frac{\pi}{z}\right)^{-1}\left(1-\frac{\pi^{q}}{z}\right)^{-1}+\cdots 1
\end{array}\right) .
$$

On cherche un anneau $\&$ tel que ${ }^{\tau}\left(R^{\prime-1} R\right)$ ait ses coefficients dans $\phi$. En fait nous allons garder la notation $\&$ pour un anneau que nous introduirons dans le paragraphe suivant et qui est vraiment analogue à celui introduit dans [14, 20]. Pour l'instant nous considérons un anneau un peu plus petit que $\phi$, et adapté seulement au cas minuscule, que nous notons $\phi_{\min }$.

On pose $\phi_{\min }=\theta \widehat{\otimes} \theta_{L}\left[\left[\frac{\pi^{q}}{z}\right]\right]$ (c'est-à-dire le quotient de $\theta \widehat{\otimes} \theta_{L}[[v]]$ par l'idéal engendré $\left.\operatorname{par} z v-\pi^{q}\right)$. Soient $\left(M, \phi_{M}\right)$ et $\left(M^{\prime}, \phi_{M^{\prime}}\right)$ deux objets de la catégorie $C_{\min }$. On suppose $M$ et $M^{\prime}$ de rang $r$ sur $\theta \widehat{\otimes} \theta_{L}$ et on se donne des bases de $M$ et $M^{\prime}$ sur $\oslash \widehat{\otimes} \theta_{L}$ telles que les matrices de $\phi_{M}$ et $\phi_{M^{\prime}}$ dans ces bases soient congrues modulo $\pi$ dans $M_{r}\left(\theta \widehat{\otimes} \theta_{L}\right)$. Alors ${ }^{\tau}\left(R^{\prime-1} R\right)$ appartient à $G L_{r}\left(\phi_{\text {min }}\right)$. En effet pour tout $n \in \mathbb{N}, \phi_{M^{\prime}}{ }^{\tau} \phi_{M^{\prime}} \ldots \tau^{n-1} \phi_{M^{\prime}} \tau^{n-1} \phi_{M}^{-1} \ldots{ }^{\tau} \phi_{M}^{-1} \phi_{M}^{-1}$ appartient à $(z-\pi)^{-1} \cdots\left(z-\pi^{q^{n-1}}\right)^{-1} M_{r}\left(\Theta \widehat{\otimes} \Theta_{L}\right)$ et est congru à $R^{\prime-1} R$ modulo $\pi^{q^{n}}$. On en déduit $R^{\prime-1} R \in M_{r}\left(\theta \widehat{\otimes} \vartheta_{L}\left[\left[\frac{\pi}{z}\right]\right]\right)$ et on répète le raisonnement pour $R^{-1} R^{\prime}$. Le calcul que nous venons d'effectuer est le cas particulier où $f$ est un isomorphisme dans la proposition suivante.

Proposition 10.1. - Soient $\left(M, \phi_{M}\right)$ et $\left(M^{\prime}, \phi_{M^{\prime}}\right)$ des chtoucas locaux minuscules sur $\theta_{L}$ et $f$ un morphisme de la réduction modulo $\pi$ de $\left(M, \phi_{M}\right)$ vers la réduction modulo $\pi$ de $\left(M^{\prime}, \phi_{M^{\prime}}\right)$. Soient $\left(D, \phi_{D}\right)$ et $\left(D^{\prime}, \phi_{D^{\prime}}\right)$ les isochtoucas associés par réduction modulo $\pi_{L}$, et $h:\left(D, \phi_{D}\right) \rightarrow\left(D^{\prime}, \phi_{D^{\prime}}\right)$ obtenu à partir de $f$ par réduction modulo $\pi_{L}$. Soient $R: M \widehat{\otimes}_{\partial \widehat{\otimes}_{\vartheta_{L}}} \mathscr{C}\left[\frac{1}{\alpha}\right] \rightarrow D \widehat{\otimes}_{k((z))} \mathscr{C}\left[\frac{1}{\alpha}\right]$ comme dans le lemme 7.4 et $R^{\prime}$ associé à $M^{\prime}$. Alors l'image de

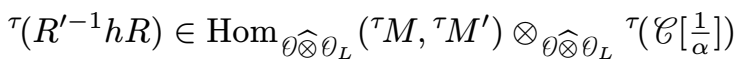

dans $\operatorname{Hom}_{\partial \widehat{\vartheta} \vartheta_{L}}\left({ }^{\tau} M,{ }^{\tau} M^{\prime}\right) \otimes_{\vartheta \widehat{\otimes} \vartheta_{L}} \phi_{\min }\left[\frac{1}{z}\right]$ appartient à $\operatorname{Hom}_{\partial \widehat{\otimes} \vartheta_{L}}\left({ }^{\tau} M,{ }^{\tau} M^{\prime}\right) \otimes_{\vartheta \widehat{\otimes} \vartheta_{L}} \&_{\min }$.

Démonstration. - Soit $\tilde{f}: M \rightarrow M^{\prime}$ un relèvement de $f$. Alors

$$
{ }^{\tau}\left(R^{\prime-1} h R\right)=\lim _{n \rightarrow \infty}{ }^{\tau} \phi_{M^{\prime}} \ldots{ }^{\tau^{n-1}} \phi_{M^{\prime}} \tau^{n}(\widetilde{f})^{\tau^{n-1}} \phi_{M}^{-1} \cdots \phi_{M}^{-1} .
$$

De plus ${ }^{\tau} \phi_{M^{\prime}} \ldots \tau^{n-1} \phi_{M^{\prime}} \tau^{n}(\widetilde{f})^{\tau^{n-1}} \phi_{M}^{-1} \cdots \phi_{M}^{-1}$ appartient à $\left(z-\pi^{q}\right)^{-1} \cdots\left(z-\pi^{q^{n-1}}\right)^{-1}$ Hom $_{\widehat{\partial} \widehat{\otimes} \vartheta_{L}}\left({ }^{\tau} M,{ }^{\tau} M^{\prime}\right)$ et est congru à ${ }^{\tau}\left(R^{\prime-1} h R\right)$ modulo $\pi^{q^{n}}$. Enfin $\left(1-\frac{\pi^{q^{i}}}{z}\right)$ est une unité dans $\phi_{\min }$ pour tout $i \in \mathbb{N}^{*}$. 
Comme tout chtouca local minuscule sur $\theta_{L} / \pi \theta_{L}$ se relève en un chtouca local minuscule sur $\theta_{L}$, on obtient ainsi un foncteur $\mathbb{S}$ de la catégorie des chtoucas locaux minuscules sur $\theta_{L} / \pi \theta_{L}$ vers la catégorie des $S_{\mathrm{min}}$-modules libres.

Proposition 10.2. - Le morphisme de $\hat{B}$ dans $\phi_{\min }$ qui envoie $\pi$ sur $z$, u sur $\pi_{L}$ et $\frac{u^{e q}}{\pi} \operatorname{sur}\left(\frac{\pi_{L}^{e}}{\pi}\right)^{q}\left(\frac{\pi^{q}}{z}\right)$ est un isomorphisme. Via cet isomorphisme, le foncteur $\mathbb{S}$ et le foncteur $\left(M, \phi_{M}\right) \rightarrow\left(\mathbb{D}\left(M, \phi_{M}\right)\right)(B, I, s, \hat{B}, \beta, \gamma)$ de la catégorie des chtoucas locaux minuscules sur $\theta_{L} / \pi \theta_{L}$ vers la catégorie des $S_{\min }$-modules libres, sont isomorphes.

Démonstration. - Le deuxième foncteur est défini comme une limite projective, mais les formules intervenant dans le complément à la construction de $\mathbb{D}$ au paragraphe 5 (qui est récapitulé dans la proposition 5.14) passent à la limite. Plus précisément on choisit

$$
\left(\widetilde{M}, \widetilde{\phi_{M}}\right)=\left(M \otimes_{\vartheta \widehat{\otimes} \vartheta_{L}, 1 \widehat{\otimes} \kappa} \oslash \widehat{\otimes} B, \phi_{M} \widehat{\otimes} 1\right)
$$

comme relèvement de la réduction modulo $\pi \theta_{L}$ de $\left(M, \phi_{M}\right)$, de $\theta_{L} / \pi \theta_{L}$ à $B=\theta \widehat{\otimes} \theta_{L}$ et on fait de même avec $\left(M^{\prime}, \phi_{M^{\prime}}\right)$. À la limite ${ }^{\tau} \mathcal{F} \underset{\left(\widetilde{M^{\prime}}, \widetilde{\phi_{M^{\prime}}}\right),\left(\widetilde{M}, \widetilde{\phi_{M}}\right)}{\widetilde{C}}(f)$ est donné par (75).

Remarque. - Soit $\left(M, \phi_{M}\right)$ dans la catégorie $C_{\min }$ et $\left(D, \phi_{D}\right)$ l'isochtouca obtenu par réduction modulo $\pi_{L}$. On a vu que ${ }^{\tau}\left(\mathscr{C}\left[\frac{1}{\alpha}\right]\right) \subset \phi_{\min }\left[\frac{1}{z}\right]$ donc $R: M \widehat{\otimes}_{\vartheta \widehat{\otimes} \vartheta_{L}} \mathscr{C}\left[\frac{1}{\alpha}\right] \rightarrow$ $D \widehat{\otimes}_{k((z))} \mathscr{C}\left[\frac{1}{\alpha}\right]$ induit un isomorphisme

$$
{ }^{\tau} R:{ }^{\tau} M \widehat{\otimes}_{\vartheta \widehat{\otimes} \vartheta_{L}} \phi_{\min }\left[\frac{1}{z}\right] \stackrel{\sim}{\longrightarrow} D \widehat{\otimes}_{k((z))} \phi_{\min }\left[\frac{1}{z}\right] .
$$

Soit $\Delta \subset D$ le $k[[z]]$-réseau obtenu par réduction de $M$ modulo $\pi_{L}$. Si $e=1$ on a

$$
{ }^{\tau} R\left({ }^{\tau} M \widehat{\otimes}_{\partial \widehat{\otimes} \vartheta_{L}} \phi_{\min }\right)={ }^{\tau} \Delta \widehat{\otimes}_{k[[z]]} \phi_{\text {min }}
$$

mais cela est faux en général si $e>1$. En effet on choisit une base de $M$, dont la réduction modulo $\pi_{L}$ fournit une base de $\Delta$. La matrice de $R$ est donnée par la formule

$$
{ }^{\tau} R=\lim _{n \rightarrow \infty}{ }^{\tau} \phi_{D} \tau^{2} \phi_{D} \ldots{ }^{n-1} \phi_{D}{ }^{n-1} \phi_{M}^{-1} \cdots{ }^{2} \phi_{M}^{-1 \tau} \phi_{M}^{-1} .
$$

Dans l'exemple où $M=\left(\Theta \widehat{\otimes} \theta_{L}\right)^{2}$ et $\phi_{M}=\left(\begin{array}{cc}z-\pi & 0 \\ \pi_{L} & 1\end{array}\right)$, on a ${ }^{\tau} R \notin G L_{2}\left(\phi_{\min }\right)$ si $e>1$, c'est-à-dire que (76) n'a pas lieu.

Nous inspirant de Faltings [20] et de Breuil [14], nous allons introduire une autre catégorie Q-linéaire $C_{\min }^{\prime}$, dont les objets sont constitués d'un isochtouca de Hodge-Pink minuscule, muni d'une «structure entière ».

Plus précisément la catégorie $C_{\min }^{\prime}$ est formée des $\left(D, \phi_{D}, V_{D}, \mathcal{M}\right)$, où

- $\left(D, \phi_{D}\right)$ est un isochtouca i.e. $D$ est un $k((z))$-espace vectoriel de dimension finie et $\phi_{D}:{ }^{\tau} D \rightarrow D$ est un isomorphisme,

- $V_{D}$ est une structure de Hodge-Pink «minuscule» c'est-à-dire que $V_{D}$ est un $L[[z-\pi]]$-module libre et qu'en posant $U_{D}={ }^{\tau} D \otimes_{k((z))} L[[z-\pi]]$, on a $U_{D} \subset V_{D} \subset(z-\pi)^{-1} U_{D}$,

- $M$ est un $\&_{\text {min }}$-module libre, muni d'un isomorphisme

$$
\mathcal{M} \otimes_{\phi_{\min }} \&_{\min }\left[\frac{1}{z}\right] \simeq{ }^{\tau} D \otimes_{k((z))} \phi_{\min }\left[\frac{1}{z}\right]
$$


- on suppose que $\mathcal{M}$ est « fortement divisible », c'est-à-dire

$$
\phi_{\min } \cdot z^{-1 \tau}\left(\left(\phi_{D} \otimes 1\right)\left(\mathcal{M} \cap(z-\pi) V_{D}\right)\right)=\mathcal{M} .
$$

Dans cette formule $\phi_{\min } .(\cdots)$ désigne le sous- $\phi_{\min }$-module engendré par $(\cdots)$ dans ${ }^{\tau} D \otimes_{k((z))} \phi_{\min }\left[\frac{1}{z}\right]$. L'intersection avec $V_{D}$ a un sens car $\phi_{\text {min }}$ est inclus dans $\mathscr{E} x t L[[z-\pi]]$. On peut remarquer que dans le cas minuscule une structure de Hodge-Pink est simplement une structure de Hodge.

Comme $\mathcal{M} \cap(z-\pi) V_{D} \supset(z-\pi) \mathcal{M}$ et $1-\frac{\pi^{q}}{z}$ est une unité de $\phi_{\text {min }}$, la condition de forte divisibilité implique que $\mathcal{M}$ est stable par $(1 \widehat{\otimes} \mathrm{Fr}) \circ\left(\phi_{D} \otimes 1\right)$ (condition demandée par Breuil dans [14]).

Dans la catégorie $C_{\min }^{\prime}$, un morphisme entre deux objets $\left(D, \phi_{D}, V_{D}, \mathcal{M}\right)$ et $\left(D^{\prime}, \phi_{D^{\prime}}, V_{D^{\prime}}, \mathcal{M}^{\prime}\right)$ est un morphisme $f$ d'isochtoucas de Hodge-Pink, tel que $\left({ }^{\tau} f \otimes 1\right)(\mathcal{M}) \subset \mathcal{M}^{\prime}$.

On note $\mathbb{D}^{+}: C_{\min } \rightarrow C_{\min }^{\prime}$ le foncteur qui à un objet $\left(M, \phi_{M}\right)$ de $C_{\min }$ associe $\left(D, \phi_{D}, V_{D}, \mathcal{M}\right)$ avec $\left(D, \phi_{D}, V_{D}\right)=\mathbb{D}_{\text {iso }}^{+}\left(M\left[\frac{1}{z}\right], \phi_{M} \otimes 1\right)$ (donc avec $V_{D}$ comme dans (48)) et avec $\mathcal{M}={ }^{\tau} M \otimes_{\vartheta \widehat{\otimes} \vartheta_{L}} \phi_{\min }$ qui est un $\phi_{\min }$-module libre muni de l'isomorphisme $\mathcal{M} \otimes_{\phi_{\min }} \phi_{\min }\left[\frac{1}{z}\right] \simeq{ }^{\tau} D \otimes_{k((z))} \phi_{\min }\left[\frac{1}{z}\right]$ associé à ${ }^{\tau} R$ (en effet on a montré ci-dessus que ${ }^{\tau} R$ induit un isomorphisme entre ${ }^{\tau} M \otimes_{\vartheta_{\widehat{\otimes} \vartheta_{L}}} \&_{\min }\left[\frac{1}{z}\right]$ et $\left.{ }^{\tau} D \otimes_{k((z))} \phi_{\min }\left[\frac{1}{z}\right]\right)$.

Montrons d'abord que le foncteur $\mathbb{D}^{+}$est bien défini. Il s'agit de montrer que $M={ }^{\tau} M \otimes_{\partial \widehat{\otimes} \vartheta_{L}} \&_{\min }$ est fortement divisible. L'inclusion

$$
\phi_{\min } \cdot z^{-1 \tau}\left(\left(\phi_{D} \otimes 1\right)\left(\mathcal{M} \cap(z-\pi) V_{D}\right)\right) \supset \mathcal{M}
$$

est évidente. En effet on a

$$
\mathcal{M}(z-\pi) V_{D} \supset{ }^{\tau} R\left({ }^{\tau} M\right) \cap(z-\pi) V_{D} \supset{ }^{\tau} R\left((z-\pi) \phi_{M}^{-1}(M)\right)
$$

puisque par définition de la catégorie $C_{\min },(z-\pi) \phi_{M}^{-1} \in \operatorname{Hom}_{\vartheta \widehat{\vartheta} \vartheta_{L}}\left(M,{ }^{\tau} M\right)$ (en fait $\left.{ }^{\tau} R\left({ }^{\tau} M\right) \cap(z-\pi) V_{D}={ }^{\tau} R\left((z-\pi) \phi_{M}^{-1}(M)\right)\right)$. Donc

$$
\begin{aligned}
z^{-1 \tau}\left(\left(\phi_{D} \otimes 1\right)\left(\mathcal{M} \cap(z-\pi) V_{D}\right)\right) & \supset z^{-1 \tau}\left(\left(\phi_{D} \otimes 1\right)\left({ }^{\tau} R\left((z-\pi) \phi_{M}^{-1}(M)\right)\right)\right) \\
& =\left(1-\frac{\pi^{q}}{z}\right)^{\tau} R\left({ }^{\tau} M\right) \text { puisque }\left(\phi_{D} \otimes 1\right)^{\tau} R \phi_{M}^{-1}=R .
\end{aligned}
$$

Enfin $1-\frac{\pi^{q}}{z}$ est inversible dans $\phi_{\min }$.

Il nous reste donc à montrer l'inclusion inverse

$$
z^{-1 \tau}\left(\left(\phi_{D} \otimes 1\right)\left(\mathcal{M} \cap(z-\pi) V_{D}\right)\right) \subset \mathcal{M} .
$$

Comme $\phi_{M}$ appartient à $\operatorname{Hom}_{\widehat{\partial} \widehat{\otimes} \vartheta_{L}}\left({ }^{\tau} M, M\right)$, et par la définition de $V_{D}$, on a

$$
\begin{aligned}
R^{-1}\left(\left(\phi_{D} \otimes 1\right)\left(\mathcal{M} \cap(z-\pi) V_{D}\right)\right) & \subset\left(M \otimes_{\partial \widehat{\vartheta}_{\theta_{L}}} \phi_{\min }\right) \cap\left((z-\pi) M \otimes_{\partial \widehat{\otimes} \vartheta_{L}} L[[z-\pi]]\right) \\
& =M \otimes_{\partial \widehat{\otimes} \theta_{L}}\left(\phi_{\min } \cap(z-\pi) L[[z-\pi]]\right) .
\end{aligned}
$$

Mais $\phi_{\min } \cap(z-\pi) L[[z-\pi]]=(z-\pi)\left(\phi_{\min }+\frac{\pi^{q-1}}{z} \phi_{\min }\right)($ l'intersection a lieu dans $L((z-\pi)))$ et donc

$$
{ }^{\tau}\left(\phi_{\min } \cap(z-\pi) L[[z-\pi]]\right) \subset\left(z-\pi^{q}\right) \phi_{\min } .
$$

Comme $\left(1-\frac{\pi^{q}}{z}\right) \in \phi_{\min }, z^{-1 \tau}\left(\phi_{\min } \cap(z-\pi) L[[z-\pi]]\right) \subset \phi_{\min }$, donc $\mathcal{M}$ est fortement divisible. Nous avons démontré que le foncteur $\mathbb{D}^{+}: C_{\min } \rightarrow C_{\min }^{\prime}$ est bien défini. 
Il est important de rappeler (comme on l'a vu, de deux manières différentes, au début du paragraphe) que $\mathcal{M}$ ne dépend que de la réduction modulo $\pi$ de $\left(M, \phi_{M}\right)$.

Il résulte de la fidélité du foncteur $\mathbb{D}_{\text {iso }}^{+}$du théorème 7.3 que le foncteur $\mathbb{D}^{+}: C_{\min } \rightarrow C_{\min }^{\prime}$ est fidèle.

ThÉorème 10.3. - Le foncteur $\mathbb{D}^{+}: C_{\min } \rightarrow C_{\min }^{\prime}$ est une équivalence de catégories, compatible, via les foncteurs d'oubli de $C_{\min }$ vers la catégorie des isochtoucas locaux sur $\theta_{L}$, et de $C_{\min }^{\prime}$ vers la catégorie des isochtoucas de Hodge-Pink, avec le foncteur $\mathbb{D}_{\text {iso }}^{+}$du théorème 7.3.

Remarque. - Il résulte du théorème 10.3 que, pour tout $\left(D, \phi_{D}, V_{D}, \mathcal{M}\right) \in C_{\min }^{\prime}$, $\left(D, \phi_{D}, V_{D}\right)$ est faiblement admissible. On a vu au début de ce paragraphe, et dans la proposition 10.2 , que $\mathbb{D}^{+}$est compatible avec le foncteur $\mathbb{D}$ du paragraphe 5 dans la mesure où $\mathcal{M}$ est la valeur en un certain pro-objet test du $\theta$-cristal $\mathbb{D}\left(M, \phi_{M}\right)$. On peut renforcer cet énoncé de compatibilité entre $\mathbb{D}^{+}$et $\mathbb{D}$ si on observe que $\mathcal{M}$ (vu comme sous- $\varnothing_{\text {min }}$-module de $\left.{ }^{\tau} D \otimes_{k((z))} \phi_{\min }\left[\frac{1}{z}\right]\right)$ est stable par les opérateurs différentiels $K$-linéaires, au sens de Grothendieck, $z^{[n / q]} \frac{1}{n !}\left(\frac{d}{d \pi_{L}}\right)^{n}$ (agissant trivialement sur ${ }^{\tau} D$ ). En effet on le vérifie pour $\left(D, \phi_{D}, V_{D}, \mathcal{M}\right) \in C_{\min }^{\prime}$ image par $\mathbb{D}^{+}$de $\left(M, \phi_{M}\right) \in C_{\min }$. D'abord $\phi_{\min }$ est stable par ces opérateurs. Donc il suffit de vérifier que $z^{[n / q]} \frac{1}{n !}\left(\frac{d}{d \pi_{L}}\right)^{n}\left({ }^{\tau}(R M)\right) \subset{ }^{\tau}(R M) \otimes_{\vartheta \widehat{\otimes} \vartheta_{L}} \phi_{\text {min }}$ dans ${ }^{\tau} D \otimes_{k((z))} \&_{\min }\left[\frac{1}{z}\right]$. Mais si $k$ est tel que $q^{k} \leq n<q^{k+1}$, on a

$$
{ }^{\tau}(R M) \subset\left(z^{-k}\right)^{\tau} \phi_{D} \cdots \tau^{\tau^{k}} \phi_{D} \tau^{k+1}(R M) \otimes_{\vartheta \widehat{\otimes} \theta_{L}} \phi_{\min }
$$

puisque $1-\frac{\pi^{q}}{z}, \ldots, 1-\frac{\pi^{q^{k}}}{z}$ sont des unités dans $\phi_{\min },{ }^{\tau} \phi_{D} \cdots \tau^{k} \phi_{D}{ }^{\tau^{k+1}}(R M)$ est stable par $\frac{1}{n !}\left(\frac{d}{d \pi_{L}}\right)^{n},{ }^{\tau} \phi_{D} \ldots{ }^{\tau^{k}} \phi_{D}{ }^{\tau^{k+1}}(R M) \subset{ }^{\tau}(R M)$, et enfin $[n / q] \geq q^{k-1} \geq k$. Or, en utilisant la formule de Taylor et en procédant comme dans le paragraphe IV.1.6 de [4], on peut montrer que la catégorie des $\theta$-cristaux relativement à $\left(\theta, \pi \theta, \gamma_{\theta}\right) \operatorname{sur} \theta_{L}$ (ou sur $\left.\theta_{L} / \pi \theta_{L}\right)$ est équivalente à celle des $\phi_{\min }$-modules libres munis d'une action des opérateurs différentiels $z^{[n / q]} \frac{1}{n !}\left(\frac{d}{d \pi_{L}}\right)^{n}$.

Début de la démonstration du théorème 10.3. - La compatibilité est évidente. Montrons d'abord que $\mathbb{D}^{+}$est pleinement fidèle. Soient $\left(M, \phi_{M}\right)$ et $\left(M^{\prime}, \phi_{M^{\prime}}\right)$ deux objets de $C_{\min }$ et $\left(D, \phi_{D}, V_{D}, \mathcal{M}\right)$ et $\left(D^{\prime}, \phi_{D^{\prime}}, V_{D^{\prime}}, \mathcal{M}^{\prime}\right)$ leurs images dans $C_{\min }^{\prime}$. On veut montrer la surjectivité de l'application

$$
\operatorname{Hom}_{C_{\text {min }}}\left(\left(M, \phi_{M}\right),\left(M^{\prime}, \phi_{M^{\prime}}\right)\right) \rightarrow \operatorname{Hom}_{C_{\min }^{\prime}}\left(\left(D, \phi_{D}, V_{D}, \mathcal{M}\right),\left(D^{\prime}, \phi_{D^{\prime}}, V_{D^{\prime}}, \mathcal{M}^{\prime}\right)\right)
$$

dont nous venons de voir l'injectivité.

Soit $h \in \operatorname{Hom}_{C_{\min }^{\prime}}\left(\left(D, \phi_{D}, V_{D}, \mathcal{M}\right),\left(D^{\prime}, \phi_{D^{\prime}}, V_{D^{\prime}}, \mathcal{M}^{\prime}\right)\right)$. Par la pleine fidélité de $\mathbb{D}_{\text {iso }}^{+}$, nous savons qu'il existe un unique morphisme $f$ d'isochtoucas locaux dans $\operatorname{Hom}_{\vartheta \widehat{\otimes} \vartheta_{L}}\left(M, M^{\prime}\right) \otimes_{\partial \widehat{\otimes} \vartheta_{L}} \oslash \widehat{\otimes} \vartheta_{L}\left[\frac{1}{z}\right]$ qui vérifie $f \circ\left(\phi_{M} \otimes 1\right)=\left(\phi_{M}^{\prime} \otimes 1\right) \circ{ }^{\tau} f$ et dont la réduction modulo $\pi_{L}$ est $h$. L'hypothèse $(h \otimes 1)(\mathcal{M}) \subset \mathcal{M}^{\prime}$ implique que $f \otimes_{\vartheta \widehat{\otimes} \vartheta_{L}\left[\frac{1}{z}\right]} 1_{\oint_{\min }\left[\frac{1}{z}\right]}$ envoie ${ }^{\tau} M \otimes_{\vartheta \widehat{Q} \vartheta_{L}} \phi_{\min }$ dans ${ }^{\tau} M^{\prime} \otimes_{\vartheta \widehat{\otimes} \vartheta_{L}} \phi_{\min }$. Il s'agit de montrer que $f$ appartient à $\operatorname{Hom}_{\widehat{\partial \otimes} \vartheta_{L}}\left(M, M^{\prime}\right)$. En fait c'est une conséquence de la proposition 4.1 : comme $\theta \widehat{\otimes}\left(\theta_{L} / \pi^{q} \theta_{L}\right)$ est un quotient de $\phi_{\min }, \mathcal{M}$ détermine la réduction de ${ }^{\tau} M$ modulo $\pi^{q} \vartheta_{L}$, donc la réduction de $M$ modulo $\pi \theta_{L}$, et comme $\pi^{q-2}$ est nilpotent dans $B$ (grâce à l'hypothèse $q \geq 3$ ), la proposition 4.1 implique que $\left(M, \phi_{M}\right)$ est déterminé par sa classe d'isogénie et par la réduction de $M$ modulo $\pi \theta_{L}$ (c'est-à-dire que le foncteur qui à $\left(M, \phi_{M}\right)$ 
associe l'isochtouca local $\left(M\left[\frac{1}{z}\right], \phi_{M} \otimes 1\right)$ et la réduction de $M$ modulo $\pi \theta_{L}$ est pleinement fidèle). Nous rappelons l'argument dans ce cas particulier pour la commodité du lecteur.

Choisissons des bases de $M$ et $M^{\prime}$ sur $\theta \widehat{\otimes} \theta_{L}$ et notons $r$ et $r^{\prime}$ les rangs de $M$ et $M^{\prime}$. On a donc $f \in M_{r^{\prime} r}\left(\theta \widehat{\otimes} \theta_{L}\left[\frac{1}{z}\right]\right)$ et ${ }^{\tau} f \in M_{r^{\prime} r}\left(\phi_{\min }\right)$ et il s'agit de montrer $f \in M_{r^{\prime} r}\left(\theta \widehat{\otimes} \theta_{L}\right)$. Comme $\theta \widehat{\otimes}\left(\theta_{L} / \pi^{q} \theta_{L}\right)$ est un quotient de $\phi_{\text {min }}$, l'hypothèse ${ }^{\tau} f \in M_{r^{\prime} r}\left(\phi_{\text {min }}\right)$ implique que $f$ modulo $\pi$ appartient à $M_{r^{\prime} r}\left(\theta \widehat{\otimes}\left(\theta_{L} / \pi \theta_{L}\right)\right)$.

On a $f=\phi_{M^{\prime}}{ }^{\tau} f \phi_{M}^{-1}$. Supposons par l'absurde que $f$ n'appartient pas à $M_{r^{\prime} r}\left(\theta \widehat{\otimes} \theta_{L}\right)$. Soit $a \in \mathbb{N}$ le petit entier tel que $z^{a} f$ appartienne à $M_{r^{\prime} r}\left(\theta \widehat{\otimes} \theta_{L}\right)$. Par hypothèse $a>0$. Soit $b \in \mathbb{N}$ le plus grand entier tel que $z^{a} f \bmod z \in M_{r^{\prime} r}\left(\theta_{L}\right)$ appartienne à $\pi_{L}^{b} M_{r^{\prime} r}\left(\theta_{L}\right)$. Par hypothèse, $b \geq e$. On a l'égalité suivante, où toutes les matrices entre parenthèses ont leurs coefficients dans $\theta \widehat{\otimes} \theta_{L}$ :

$$
(z-\pi)\left(z^{a} f\right)=\left(\phi_{M^{\prime}}\right)\left(z^{a \tau} f\right)\left((z-\pi) \phi_{M}^{-1}\right) .
$$

En réduisant cette égalité modulo $z$, on trouve l'égalité suivante, où toutes les matrices entre parenthèses sont à coefficients dans $\theta_{L}$ :

$$
(-\pi)\left(\left(z^{a} f\right) \bmod z\right)=\left(\phi_{M^{\prime}} \bmod z\right)\left(\left(z^{a \tau} f\right) \bmod z\right)\left((z-\pi) \phi_{M}^{-1} \bmod z\right) .
$$

Or $\left(z^{a} f\right) \bmod z$ est divisible exactement $\operatorname{par} \pi_{L}^{b}$ et $\left(z^{a}{ }^{\tau} f\right) \bmod z$ est divisible (exactement) $\operatorname{par} \pi_{L}^{q b}$. Comme $\pi / \pi_{L}^{e}$ est une unité, le membre de gauche est divisible exactement par $\pi_{L}^{b+e}$, alors que le membre de droite est divisible par $\pi_{L}^{q b}$. Comme $b \geq e$ et $q \geq 3$ on a $b(q-1)>e$ ce qui amène une contradiction.

REMARQUe. - Voici un contre-exemple qui montre qu'on ne peut pas espérer un foncteur pleinement fidèle pour $e=1$ et $q=2$ (on note que le calcul qui suit est en caractéristique 2). On prend $M=M^{\prime}=\left(\theta \widehat{\otimes} \theta_{L}\right)^{2}, \phi_{M}=\left(\begin{array}{cc}1 & 0 \\ 0 & z-\pi\end{array}\right)$ et

$$
\phi_{M^{\prime}}=\left(\begin{array}{cc}
1 & \pi / z \\
0 & 1
\end{array}\right) \phi_{M}\left(\begin{array}{cc}
1 & \pi^{2} / z \\
0 & 1
\end{array}\right)^{-1}=\left(\begin{array}{cc}
1 \frac{\pi^{2}+\pi(z-\pi)}{z} \\
0 & z-\pi
\end{array}\right)=\left(\begin{array}{cc}
1 & \pi \\
0 & z-\pi
\end{array}\right) .
$$

Alors $\left(M, \phi_{M}\right)$ et $\left(M^{\prime}, \phi_{M^{\prime}}\right)$ sont des objets de $C_{\min }$ et $f=\left(\begin{array}{cc}1 & \pi / z \\ 0 & 1\end{array}\right)$ est une quasi-isogénie congrue à Id modulo $\pi$, de $M$ vers $M^{\prime}: z f$ est un morphisme de $M$ vers $M^{\prime}$ dans $C_{\min }$ mais ce n'est pas le cas de $f$, à cause du dénominateur $z$ dans $\pi / z$. On voit que ${ }^{\tau} f$ appartient à $G L_{2}\left(\phi_{\min }\right)$, donc $f$ définit un morphisme du quadruplet $\left(D, \phi_{D}, V_{D}, \mathcal{M}\right)$ associé à $M$ vers le quadruplet $\left(D^{\prime}, \phi_{D^{\prime}}, V_{D^{\prime}}, \mathcal{M}^{\prime}\right)$ associé à $M^{\prime}$.

Suite de la démonstration du théorème 10.3. - Il nous reste à montrer que pour $q \geq 3$ le foncteur de $C_{\min }$ dans $C_{\min }^{\prime}$ est essentiellement surjectif. On doit construire un $\vartheta \widehat{\otimes} \vartheta_{L}$-module libre $M$ de rang $r$, à partir de la donnée d'un $\phi_{\min }$-module libre $\mathcal{M}$ de rang $r$. L'idée est de construire une suite de modules libres de rang $r$ sur des anneaux qui se rapprochent de plus en plus de $\theta \widehat{\otimes} \theta_{L}$ et de définir $M$ comme la limite de cette suite. La difficulté de cette démonstration n'est pas de construire un $\theta \widehat{\otimes} \theta_{L}$-module $M$, mais de montrer qu'il est libre de rang $r$. Il est important de noter la parenté entre la preuve qui suit et la démonstration du théorème «faiblement admissible implique admisible » donnée au 
paragraphe 8 . Nous commençons par un lemme qui montre que, bien que le lemme 8.1 soit faux pour $\theta \widehat{\otimes} \theta_{L}\left[\left[\frac{\pi^{a}}{z}\right]\right]$ (qui n'est pas régulier), une certaine approximation reste vraie.

Lemme 10.4. - a) Soient $a \in \mathbb{N}, a \geq 3$, et $\mathcal{N}$ un $\oslash \widehat{\otimes} \vartheta_{L}\left[\left[\frac{\pi^{a}}{z}\right]\right]$-module libre et $W$ un sous- $L[[z-\pi]]-$ module de $\mathcal{N} \otimes_{\oslash \widehat{\otimes}_{\vartheta_{L}}\left[\left[\frac{\pi^{a}}{z}\right]\right]} L[[z-\pi]]$ contenant $(z-\pi) \mathcal{N} \otimes_{\oslash \widehat{\otimes} \vartheta_{L}\left[\left[\frac{\pi^{a}}{z}\right]\right]} L[[z-\pi]]$. Alors $\mathcal{N}^{\prime}=\Theta \widehat{\otimes} \vartheta_{L}\left[\left[\frac{\pi^{a-1}}{z}\right]\right] .(\mathcal{N} \cap W)$ est un sous- $\Theta \widehat{\otimes} \theta_{L}\left[\left[\frac{\pi^{a-1}}{z}\right]\right]-$ module libre de $\mathcal{N} \otimes_{\partial \widehat{\otimes} \vartheta_{L}\left[\left[\frac{\pi^{a}}{z}\right]\right]} \oslash \widehat{\otimes} \vartheta_{L}\left[\left[\frac{\pi^{a-1}}{z}\right]\right]$,

$$
\begin{gathered}
\mathcal{N}^{\prime} \otimes_{\partial \widehat{\otimes} \vartheta_{L}\left[\left[\frac{\pi^{a-1}}{z}\right]\right]} \oslash \widehat{\otimes} \vartheta_{L}\left[\left[\frac{\pi^{a-1}}{z}\right]\right]\left[\frac{1}{z-\pi}\right]=\mathcal{N} \otimes_{\vartheta \widehat{\otimes} \vartheta_{L}\left[\left[\frac{\pi^{a}}{z}\right]\right]} \oslash \widehat{\otimes} \vartheta_{L}\left[\left[\frac{\pi^{a-1}}{z}\right]\right]\left[\frac{1}{z-\pi}\right] \\
\text { et } \quad \mathcal{N}^{\prime} \otimes_{\partial \widehat{\otimes} \vartheta_{L}\left[\left[\frac{\pi^{a-1}}{z}\right]\right]} L[[z-\pi]]=W .
\end{gathered}
$$

De plus

$$
\operatorname{det}\left(\mathcal{N}^{\prime}\right)=(z-\pi)^{k} \operatorname{det}(\mathcal{N}) \otimes_{\vartheta \widehat{\otimes} \vartheta_{L}\left[\left[\frac{\pi^{a}}{z}\right]\right]} \oslash \widehat{\otimes} \vartheta_{L}\left[\left[\frac{\pi^{a-1}}{z}\right]\right]
$$

où $k=\operatorname{dim}_{L}\left(\mathcal{N} \otimes_{\partial \widehat{\otimes} \vartheta_{L}\left[\left[\frac{\pi^{a}}{z}\right]\right]} L[[z-\pi]] / W\right)$.

b) Soit de plus $N$ un $\theta \widehat{\otimes} \vartheta_{L}$-module libre muni d'un isomorphisme $N \otimes_{\partial \widehat{\otimes} \theta_{L}} \theta \widehat{\otimes} \vartheta_{L}\left[\left[\frac{\pi^{a}}{z}\right]\right]=\mathcal{N}$. Alors l'inclusion

$$
\theta \widehat{\otimes} \vartheta_{L}\left[\left[\frac{\pi^{a-1}}{z}\right]\right] .(N \cap W) \subset \theta \widehat{\otimes} \theta_{L}\left[\left[\frac{\pi^{a-1}}{z}\right]\right] .(\mathcal{N} \cap W)
$$

est une égalité.

c) Soient de plus $b \geq a$ et $\widetilde{\mathcal{N}}$ un $\oslash \widehat{\otimes} \vartheta_{L}\left[\left[\frac{\pi^{b}}{z}\right]\right]$-module libre muni d'un isomorphisme $\widetilde{\mathcal{N}} \otimes_{\vartheta \widehat{\otimes} \vartheta_{L}\left[\left[\frac{\pi^{b}}{z}\right]\right]} \oslash \widehat{\otimes} \vartheta_{L}\left[\left[\frac{\pi^{a}}{z}\right]\right]=\mathcal{N}$. Alors l'inclusion

$$
\theta \widehat{\otimes} \vartheta_{L}\left[\left[\frac{\pi^{a-1}}{z}\right]\right] .(\widetilde{\mathcal{N}} \cap W) \subset \theta \widehat{\otimes} \vartheta_{L}\left[\left[\frac{\pi^{a-1}}{z}\right]\right] .(\mathcal{N} \cap W)
$$

est une égalité.

Démonstration. - On montre simultanément a) et b). Soit $N$ un $\Theta \widehat{\otimes} \Theta_{L}$-module libre muni d'un isomorphisme $N \otimes_{\vartheta \widehat{\otimes} \vartheta_{L}} \oslash \widehat{\otimes} \theta_{L}\left[\left[\frac{\pi^{a}}{z}\right]\right]=\mathcal{N}$. D'après le lemme 8.1, $N^{\prime}=N \cap W$ est un $\theta \widehat{\otimes} \theta_{L}$-module libre, et on a

$$
(z-\pi) N \subset N^{\prime} \subset N, \operatorname{det}\left(N^{\prime}\right)=(z-\pi)^{k} \operatorname{det}(N) \text { et } W=N^{\prime} \otimes_{\vartheta \widehat{\otimes} \theta_{L}} L[[z-\pi]] .
$$

Comme $\mathcal{N}=N \otimes_{\oslash \widehat{\otimes} \vartheta_{L}} \oslash \widehat{\otimes} \vartheta_{L}\left[\left[\frac{\pi^{a}}{z}\right]\right]$ il résulte de ce qui précède que

$$
\mathcal{N} \cap W \subset N^{\prime} \otimes_{\vartheta \widehat{\otimes} \vartheta_{L}}\left((z-\pi)^{-1} \oslash \widehat{\otimes} \vartheta_{L}\left[\left[\frac{\pi^{a}}{z}\right]\right] \cap L[[z-\pi]]\right) .
$$

On vérifie facilement que

$$
\begin{aligned}
(z-\pi)^{-1} \oslash \widehat{\otimes} \vartheta_{L}\left[\left[\frac{\pi^{a}}{z}\right]\right] \cap L[[z-\pi]] & =\theta \widehat{\otimes} \vartheta_{L}\left[\left[\frac{\pi^{a}}{z}\right]\right]+\frac{\pi^{a-1}}{z} \Theta \widehat{\otimes} \vartheta_{L}\left[\left[\frac{\pi^{a}}{z}\right]\right] \\
& \subset \ominus \widehat{\otimes} \vartheta_{L}\left[\left[\frac{\pi^{a-1}}{z}\right]\right] .
\end{aligned}
$$

Donc

$$
\mathcal{N}^{\prime} \subset N^{\prime} \otimes_{\vartheta \widehat{\otimes} \vartheta_{L}} \oslash \widehat{\otimes} \vartheta_{L}\left[\left[\frac{\pi^{a-1}}{z}\right]\right]
$$


et on a l'égalité puisque $\mathcal{N} \cap W$ contient évidemment $N^{\prime}$. Enfin

$$
\operatorname{det}\left(\mathcal{N}^{\prime}\right)=\operatorname{det}\left(N^{\prime}\right) \otimes_{\vartheta \widehat{\otimes} \vartheta_{L}} \oslash \widehat{\otimes} \vartheta_{L}\left[\left[\frac{\pi^{a-1}}{z}\right]\right] .
$$

Pour montrer c) on applique b) en prenant $N$ engendré par les vecteurs d'une base de $\widetilde{\mathcal{N}}$ sur $\theta \widehat{\otimes} \theta_{L}\left[\left[\frac{\pi^{b}}{z}\right]\right]$.

Fin de la démonstration du théorème 10.3. - Montrons maintenant que le foncteur $\mathbb{D}^{+}: C_{\min } \rightarrow C_{\min }^{\prime}$ est essentiellement surjectif. Soit $\left(D, \phi_{D}, V_{D}, \mathcal{M}\right)$ un objet de $C_{\min }^{\prime}$. D'après le a) du lemme 10.4, $\oslash \widehat{\otimes} \vartheta_{L}\left[\left[\frac{\pi^{q-1}}{z}\right]\right] .\left(\mathcal{M} \cap(z-\pi) V_{D}\right)$ est un $\Theta \widehat{\otimes} \vartheta_{L}\left[\left[\frac{\pi^{q-1}}{z}\right]\right]$-module libre. On note $M_{0}$ son image $\operatorname{par}(z-\pi)^{-1}\left(\phi_{D} \otimes 1\right)$, si bien que

$$
M_{0}=\theta \widehat{\otimes} \vartheta_{L}\left[\left[\frac{\pi^{q-1}}{z}\right]\right] \cdot(z-\pi)^{-1}\left(\phi_{D} \otimes 1\right)\left(\mathcal{M} \cap(z-\pi) V_{D}\right)
$$

est un $\oslash \widehat{\otimes} \theta_{L}\left[\left[\frac{\pi^{q-1}}{z}\right]\right]$-module libre naturellement inclus dans

$$
(z-\pi)^{-1} D \otimes_{k((z))} \oslash \widehat{\otimes} \theta_{L}\left[\left[\frac{\pi^{q-1}}{z}\right]\right]\left[\frac{1}{z}\right] .
$$

Alors ${ }^{\tau} M_{0}$ est un $\theta \widehat{\otimes} \theta_{L}\left[\left[\frac{\pi^{q(q-1)}}{z}\right]\right]$-module libre qui est naturellement inclus dans $\left(z-\pi^{q}\right)^{-1}{ }^{\tau} D \otimes_{k((z))} \oslash \widehat{\otimes} \vartheta_{L}\left[\left[\frac{\pi^{q(q-1)}}{z}\right]\right]\left[\frac{1}{z}\right]$. Par l'hypothèse de forte divisibilité de $\mathcal{M}$, et comme $1-\frac{\pi^{q}}{z}$ est une unité de $\phi_{\min }$, on a

$$
{ }^{\tau} M_{0} \otimes_{\partial \widehat{\otimes} \theta_{L}\left[\left[\frac{\pi^{q(q-1)}}{z}\right]\right]} \phi_{\min }=\mathcal{M} .
$$

On pose ensuite

$$
M_{1}=\Theta \widehat{\otimes} \vartheta_{L}\left[\left[\frac{\pi^{q(q-1)-1}}{z}\right]\right] .(z-\pi)^{-1}\left(\phi_{D} \otimes 1\right)\left({ }^{\tau} M_{0} \cap(z-\pi) V_{D}\right) .
$$

Par le a) du lemme 10.4 c'est un $\Theta \widehat{\otimes} \vartheta_{L}\left[\left[\frac{\pi^{q(q-1)-1}}{z}\right]\right]$-module libre, naturellement inclus dans $(z-\pi)^{-1}\left(z-\pi^{q}\right)^{-1} D \otimes_{k((z))} \oslash \widehat{\otimes} \Theta_{L}\left[\left[\frac{\pi^{q(q-1)-1}}{z}\right]\right]\left[\frac{1}{z}\right]$. On définit par récurrence $M_{n}$ pour $n \in \mathbb{N}$ en posant

$$
M_{n+1}=\theta \widehat{\otimes} \vartheta_{L}\left[\left[\frac{\pi^{m_{n+1}}}{z}\right]\right] \cdot(z-\pi)^{-1}\left(\phi_{D} \otimes 1\right)\left({ }^{\tau} M_{n} \cap(z-\pi) V_{D}\right),
$$

où la suite $m_{n}$ est définie par $m_{0}=q-1$ et $m_{n+1}=q m_{n}-1$ (cette suite est strictement croissante et tend vers l'infini).

Par le a) du lemme 10.4, $M_{n}$ est un $\theta \widehat{\otimes} \theta_{L}\left[\left[\frac{\pi^{m_{n}}}{z}\right]\right]$-module libre, inclus dans $(z-\pi)^{-1} \cdots\left(z-\pi^{q^{n}}\right)^{-1} D \otimes_{k((z))} \Theta \widehat{\otimes} \vartheta_{L}\left[\left[\frac{\pi^{m_{n}}}{z}\right]\right]\left[\frac{1}{z}\right]$. L'hypothèse de forte divisibilité de $\mathcal{M}$, et le c) du lemme 10.4 (appliqué à $a=q, b=q m_{n-1}, \mathcal{N}=\mathcal{M}$ et $\widetilde{\mathcal{N}}={ }^{\tau} M_{n-1}$ ) montrent, par récurrence sur $n$,

$$
{ }^{\tau} M_{n} \otimes_{\partial \widehat{\otimes} \vartheta_{L}\left[\left[\frac{\pi q m_{n}}{z}\right]\right]} \phi_{\min }=\mathcal{M} .
$$

On a déjà vu que ${ }^{\tau} M_{0} \subset \mathcal{M}$. On en déduit $M_{1} \subset M_{0}$. Cette assertion a un sens car les deux sont plongés dans $(z-\pi)^{-1} D \otimes_{k((z))} \oslash \widehat{\otimes} \vartheta_{L}\left[\left[\frac{\pi^{q-1}}{z}\right]\right]\left[\frac{1}{z}\right]$. On en déduit, pour tout $n \in \mathbb{N}, M_{n+1} \subset M_{n}$. En fait l'égalité ${ }^{\tau} M_{0} \otimes_{\partial \widehat{\otimes} \vartheta_{L}\left[\left[\frac{\pi^{q(q-1)}}{z}\right]\right]} \phi_{\min }=\mathcal{M}$ implique, grâce au c) du lemme 10.4 (appliqué à $a=q, b=q m_{0}, \mathcal{N}=\mathcal{M}$ et $\widetilde{\mathcal{N}}={ }^{\tau} M_{0}$ ), que l'inclusion $M_{1} \subset M_{0}$ induit une égalité $M_{1} \otimes_{\widehat{\vartheta} \widehat{\otimes} \vartheta_{L}\left[\left[\frac{\pi^{m_{1}}}{z}\right]\right]} \oslash \widehat{\otimes} \vartheta_{L}\left[\left[\frac{\pi^{m_{0}}}{z}\right]\right]=M_{0}$. On montre alors, par 
récurrence sur $n$, grâce au c) du lemme 10.4 (appliqué à $a=q m_{n-1}, b=q m_{n}, \mathcal{N}={ }^{\tau} M_{n-1}$ et $\widetilde{\mathcal{N}}={ }^{\tau} M_{n}$ ), que l'inclusion $M_{n+1} \subset M_{n}$ induit une égalité

$$
M_{n+1} \otimes_{\oslash \widehat{\otimes} \vartheta_{L}\left[\left[\frac{\pi^{m_{n+1}}}{z}\right]\right]} \oslash \widehat{\otimes} \vartheta_{L}\left[\left[\frac{\pi^{m_{n}}}{z}\right]\right]=M_{n} .
$$

En particulier, comme $m_{n+1}>m_{n}>q^{n}$, l'inclusion $M_{n+1} \subset M_{n}$ induit une égalité modulo $\pi^{q^{n}}$, c'est-à-dire

$$
M_{n+1} \otimes_{\partial \widehat{\otimes} \vartheta_{L}\left[\left[\frac{\pi^{m_{n+1}}}{z}\right]\right]} \oslash \widehat{\otimes} \vartheta_{L} / \pi^{q^{n}}=M_{n} \otimes_{\partial \widehat{\otimes} \vartheta_{L}\left[\left[\frac{\pi^{m_{n}}}{z}\right]\right]} \oslash \widehat{\otimes} \vartheta_{L} / \pi^{q^{n}} .
$$

On prend alors $M$ égal à l'intersection des $M_{n}$.

On remarque que $Q_{n}=M_{n} \otimes_{\vartheta \widehat{\otimes} \vartheta_{L}\left[\left[\frac{\pi^{m_{n}}}{z}\right]\right]} \theta \widehat{\otimes} \theta_{L} / \pi^{q^{n}}$ est un $\Theta \widehat{\otimes} \vartheta_{L} / \pi^{q^{n}}$-module libre de rang $r$ et que $Q_{n}=Q_{n+1} \otimes_{\vartheta \widehat{\otimes} \vartheta_{L} / \pi^{q^{n+1}}} \theta \widehat{\otimes} \vartheta_{L} / \pi^{q^{n}}$. On a aussi $M=\lim _{\leftarrow} Q_{n}$ donc $M$ est un $\theta \widehat{\otimes} \theta_{L}$-module libre de rang $r$. De plus pour tout $n \in \mathbb{N}$ l'inclusion $M \subset M_{n}$ induit une égalité $M \otimes_{\vartheta \widehat{\otimes} \vartheta_{L}} \oslash \widehat{\otimes} \vartheta_{L}\left[\left[\frac{\pi^{m_{n}}}{z}\right]\right]=M_{n}$.

Comme $M_{n+1}=\Theta \widehat{\otimes} \vartheta_{L}\left[\left[\frac{\pi^{m_{n+1}}}{z}\right]\right] .(z-\pi)^{-1}\left(\phi_{D} \otimes 1\right)\left({ }^{\tau} M_{n} \cap(z-\pi) V_{D}\right)$ pour tout $n \in \mathbb{N}$, et grâce à b) du lemme 10.4, on a

$$
M=(z-\pi)^{-1}\left(\phi_{D} \otimes 1\right)\left({ }^{\tau} M \cap(z-\pi) V_{D}\right) .
$$

On définit $\psi_{M}: M \rightarrow{ }^{\tau} M$ comme la restriction de $(z-\pi)\left(\phi_{D} \otimes 1\right)^{-1}$ à $M$. On a $\phi_{M}=(z-\pi) \psi_{M}^{-1} \in \operatorname{Hom}_{\hat{\vartheta} \widehat{\otimes} \vartheta_{L}}\left({ }^{\tau} M, M\right)$. Comme

$$
M \otimes_{\partial \widehat{\otimes} \vartheta_{L}} \oslash \widehat{\otimes} \vartheta_{L}\left[\left[\frac{\pi^{m_{n}}}{z}\right]\right]=M_{n} \text { et }{ }^{\tau} M_{n} \otimes_{\vartheta \widehat{\otimes} \vartheta_{L}\left[\left[\frac{\pi^{q} m_{n}}{z}\right]\right]} \phi_{\min }=\mathcal{M}
$$

(pour n'importe quelle valeur de $n$ ) on a ${ }^{\tau} M \otimes_{\widehat{\partial} \widehat{\otimes} \vartheta_{L}} \&_{\min }=\mathcal{M}$.

On a donc montré que le foncteur $\mathbb{D}^{+}: C_{\min } \rightarrow C_{\min }^{\prime}$ est essentiellement surjectif.

Nous terminons ce paragraphe par deux remarques.

Dans le paragraphe suivant nous considérerons des chtoucas locaux non nécessairement minuscules, d'amplitude $\subset[0, m]$. Le cas particulier du théorème 11.9 où $m=1$ est quasiment équivalent au théorème 10.3 (et les foncteurs qui y figurent sont compatibles). La seule différence est que le théorème 11.9 utilise un anneau $\phi \supset \phi_{\min }$ plutôt que $\phi_{\min }$. Cet anneau $\&$ est adapté aux puissances divisées de Grothendieck et Berthelot alors que l'anneau $\phi_{\min }$ est adapté aux puissances divisées de Honda et Gross-Hopkins. Comme $\&$ est l'analogue en égales caractéristiques de l'anneau considéré dans [14, 20], nous renvoyons au paragraphe suivant pour la comparaison avec les conjectures de [14].

Lorsque $e \leq q-2$, l'idéal $\pi_{L} \theta_{L} \subset \theta_{L}$ admet des puissances divisées au sens de la définition 6.1, qui sont topologiquement nilpotentes. Soit $C_{\min }^{\prime \prime}$ la catégorie des quadruplets $\left(D, \phi_{D}, V_{D}, \Delta\right)$ avec $\left(D, \phi_{D}\right)$ un isochtouca, $V_{D}$ une structure de Hodge-Pink minuscule, et $\Delta$ un $k[[z]]$-réseau de $D$ tel que, en notant

$$
\mathrm{Fil}^{1}=\left((z-\pi) V_{D} \cap U_{D}\right) /(z-\pi) U_{D} \subset U_{D} /(z-\pi) U_{D}={ }^{\tau} D \otimes_{k((z))} L
$$

on ait égalité entre les deux sous- $k$-espaces vectoriels suivants de ${ }^{\tau} \Delta / z^{\tau} \Delta$ :

$$
\left({ }^{\tau} \Delta \otimes_{k[[z]]} \theta_{L}\right) \cap \mathrm{Fil}^{1}+\pi_{L}\left({ }^{\tau} \Delta \otimes_{k[[z]]} \theta_{L}\right) / \pi_{L}\left({ }^{\tau} \Delta \otimes_{k[[z]]} \theta_{L}\right)=z \phi_{D}^{-1}(\Delta) / z^{\tau} \Delta .
$$

Il résulte de la proposition 6.3 que le foncteur de la catégorie des chtoucas locaux minuscules sur $\theta_{L}$ vers $C_{\min }^{\prime \prime}$ qui à $\left(M, \phi_{M}\right)$ associe $\left(D, \phi_{D}, V_{D}, \Delta\right)$ avec 
$\left(D, \phi_{D}, V_{D}\right)=\mathbb{D}_{\text {iso }}^{+}\left(M\left[\frac{1}{z}\right], \phi_{M} \otimes 1\right)$ et $\Delta$ égal au réseau $M / \pi_{L} M$ de $D=\left(M / \pi_{L} M\right)\left[\frac{1}{z}\right]$, est une équivalence de catégorie. En inégales caractéristiques cette équivalence de catégories est bien connue (voir [28, 48], la proposition 5.1 du paragraphe IV de [22] et [41]). Nous allons voir que le théorème 10.3 (ou une variante de celui-ci pour $e \in\{2, \ldots, q-2\}$ ) permet de retrouver cette équivalence de catégorie et donne un algorithme un peu différent de celui figurant dans la preuve de la proposition 6.3 pour le foncteur inverse.

Si $e=1$ le lien avec le théorème 10.3 est évident, car on a alors ${ }^{\tau} R \in G L_{r}\left(\phi_{\text {min }}\right)$, donc $\mathcal{M}={ }^{\tau} M \otimes_{\partial \widehat{\otimes} \vartheta_{L}} \phi_{\min }$ est égal (dans $\left.{ }^{\tau} D \otimes_{k((z))} \phi_{\min }\left[\frac{1}{z}\right]\right) \grave{a ̀ ~}^{\tau} \Delta \otimes_{k[[z]]} \phi_{\min }$. Donc on peut remplacer la donnée de $\mathcal{M}$ par celle de $\Delta$ et la condition de forte divisiblité de $\mathcal{M}$ équivaut à (78).

Supposons maintenant $e \in\{2, \ldots, q-2\}$. On n'a pas en général $\mathcal{M}={ }^{\tau} M \otimes_{\partial_{\widehat{Q} \vartheta_{\vartheta_{L}}} \phi_{\min }}$, c'est pourquoi on va considérer une variante de l'énoncé du théorème 10.3 où l'on remplace $\phi_{\text {min }}$ par un anneau plus gros $\phi^{\prime}$. On cherche un objet test «topologique » $(B, I, s, \hat{B}, \beta, \gamma)$ relativement à $\left(\theta, \pi \theta, \gamma_{\vartheta}\right)$ sur $k$. On prend $B=(\theta \widehat{\otimes} k)[[u]], I=(\pi, u), \hat{B}=(\theta \widehat{\otimes} k)\left[\left[u, \frac{u^{q}}{\pi}\right]\right]$, $\beta: B \rightarrow \hat{B}$ l'inclusion naturelle et $\gamma: I \rightarrow \hat{B}$ défini en posant $\gamma(u)=\frac{u^{q}}{\pi}$ et $\gamma(\pi)=\pi^{q-1}$. On note $\hat{J}$ l'idéal de $\hat{B}$ engendré par $\pi$ et $\frac{u^{q}}{\pi}$ de sorte que $\left(B / I^{n}, I / I^{n}, s, \hat{B} / \hat{J}^{n}, \beta, \gamma\right)$ est un $k$-objet test relativement à $\left(\theta, \pi \theta, \gamma_{\vartheta}\right)$ au sens du paragraphe 5 . Donc

$$
\left(\mathbb{D}\left(M, \phi_{M}\right)\right)(B, I, s, \hat{B}, \beta, \gamma):=\lim _{n \in \mathbb{N}}\left(\mathbb{D}\left(M, \phi_{M}\right)\right)\left(B / I^{n}, I / I^{n}, s, \hat{B} / \hat{J}^{n}, \beta, \gamma\right)
$$

est un $\hat{B}$-module libre ne dépendant que de la réduction de $\left(M, \phi_{M}\right)$ modulo $\pi_{L}$. Dans le point de vue naif, on pose $\phi^{\prime}=\emptyset \widehat{\otimes} \vartheta_{L}\left[\left[\frac{\pi_{L}^{q}}{z}\right]\right]$. Comme dans la proposition 10.2 on a un isomorphisme de $\hat{B}$ dans $\phi^{\prime}$ qui envoie $\pi$ sur $z, u$ sur $\pi_{L}$ et $\frac{u^{q}}{\pi} \operatorname{sur} \frac{\pi_{L}^{q}}{z}$. On a alors ${ }^{\tau} M \otimes_{\vartheta \widehat{\otimes} \vartheta_{L}} \phi^{\prime}={ }^{\tau} \Delta \otimes_{k[[z]]} \phi^{\prime}$ en notant $\Delta$ le $k[[z]]$-réseau de $D$ égal à la réduction de $M$ modulo $\pi_{L}$. En effet, après avoir choisi une base de $M \operatorname{sur} \theta \widehat{\otimes} \vartheta_{L}$, la formule (77) montre que ${ }^{\tau} R$ appartient à $G L_{r}\left(\phi^{\prime}\right)$. Sous l'hypothèse $e \leq q-2$ le théorème 10.3 reste valable si on modifie la catégorie $C_{\min }^{\prime}$ en remplaçant $\phi_{\min } \operatorname{par} \phi^{\prime}$. Dans la démonstration de la pleine fidélité, on a $b(q-1)>e$ car $b \geq 1$ et $q-1>e$. Dans le lemme 10.4 il faut remplacer $\theta \widehat{\otimes} \theta_{L}\left[\left[\frac{\pi^{a}}{z}\right]\right]$ par $\theta \widehat{\otimes} \vartheta_{L}\left[\left[\frac{\pi_{L}^{a}}{z}\right]\right]$ et $\Theta \widehat{\otimes} \vartheta_{L}\left[\left[\frac{\pi^{a-1}}{z}\right]\right]$ par $\theta \widehat{\otimes} \vartheta_{L}\left[\left[\frac{\pi_{L}^{a-e}}{z}\right]\right]$. Dans la preuve $M_{n}$ est un $\theta \widehat{\otimes} \theta_{L}\left[\left[\frac{\pi_{L}^{m_{n}}}{z}\right]\right]$-module libre, où la suite $m_{n}$ est définie par $m_{0}=q-e$ et $m_{n+1}=q m_{n}-e$ et l'hypothèse $e \leq q-2$ assure que cette suite est strictement croissante et tend vers l'infini. Dans cette variante du théorème 10.3, la catégorie $C_{\min }^{\prime}$ est donc remplacée car la catégorie des quadruplets $\left(D, \phi_{D}, V_{D}, \Delta\right)$, où $\left(D, \phi_{D}, V_{D}\right)$ est un isochtouca de Hodge-Pink vérifiant $t_{N}(D)=t_{H}(D)$ et $U_{D} \subset V_{D} \subset(z-\pi)^{-1} U_{D}$, et $\Delta$ est un réseau de $D$, fortement divisible au sens où

$$
\phi^{\prime} . z^{-1 \tau}\left(\left(\phi_{D} \otimes 1\right)\left(\left({ }^{\tau} \Delta \otimes_{k[[z]]} \phi^{\prime}\right) \cap(z-\pi) V_{D}\right)\right)={ }^{\tau} \Delta \otimes_{k[[z]]} \phi^{\prime} .
$$

On vérifie facilement que cette condition équivaut à (78).

\section{Une condition de « transversalité de Griffiths »}

Ce paragraphe doit beaucoup à des discussions avec Mark Kisin.

À la fin de ce paragraphe nous montrerons le théorème 11.9, dont le but est de déterminer à isomorphisme près un chtouca local sur $\theta_{L}$, d'amplitude $\subset[0, m]$ avec $m \leq q-2$, à l'aide 
de sa classe d'isogénie et d'une structure entière qui ne dépende que de sa réduction modulo $\pi \theta_{L}$. Le paragraphe 4 montre que cela n'est pas possible en général. Nous devrons imposer une condition de «tranquillité » qui rappelle la transversalité de Griffiths, est automatique pour $m=1$ (cas minuscule) et dont l'analogue en inégales caractéristiques est toujours vérifié. Nous étudions maintenant cette condition, sans l'hypothèse $m \leq q-2$. Ensuite nous supposerons $m \leq q-2$ et nous énoncerons le théorème 11.9.

On reprend les notations du paragraphe précédent : $\theta_{L}$ un anneau de valuation discrète complet de corps résiduel $k, m_{L}$ son idéal maximal, $\pi_{L}$ une uniformisante de $\theta_{L}, L$ son corps des fractions et on suppose $\theta_{L}$ muni d'une structure de $\theta$-algèbre de sorte que l'image de $\pi$ dans $\theta_{L}$ soit un élément non nul de $m_{L}$. On note $e$ l'indice de ramification de $L$ sur $K$.

On note de plus $L_{0}=k((\pi))$, de sorte que $L$ est une extension finie de $L_{0}$. Dans ce paragraphe on supposera toujours que $L$ est une extension séparable de $L_{0}$.

Dans ce paragraphe les opérateurs différentiels seront toujours au sens de Grothendieck.

On commence par un lemme d'algèbre commutative qui est un cas particulier de la proposition 1 page 28 du chapitre 9 de [11] et qui est aussi un cas particulier des points (iii) et (iv) du théorème 28.3 de [46]. Ce lemme nous a été indiqué par Mark Kisin.

Lemme 11.1. - Soit $A$ un anneau de valuation discrète complet de corps résiduel $L$. Supposons que $L$ est une extension finie de $L_{0}$ et que A possède une structure de $L_{0}$-algèbre telle que $L_{0} \rightarrow A \rightarrow L$ soit l'inclusion de $L_{0}$ dans $L$. Si $L / L_{0}$ est séparable il existe une unique façon de prolonger $L_{0} \rightarrow A$ en $L \rightarrow A$ de sorte que $L \rightarrow A \rightarrow L$ soit l'identité.

Nous appliquons ce lemme à $L_{0}$ et $L$ comme ci-dessus et $A=L[[z-\pi]]$. Le point important est que $A$ est muni de la structure de $L_{0}$-algèbre telle que l'image de $\pi$ soit $z=\pi+(z-\pi)$. Par le lemme précédent cette structure se prolonge de manière unique en une structure de $L$-algèbre $\operatorname{sur} A$. On notera $\iota: L \rightarrow L[[z-\pi]]$ le morphisme associé. Voici un exemple : si $\pi=\pi_{L}^{e}$ avec $e$ premier à $q, \iota\left(\pi_{L}\right)$ doit être

$$
z^{\frac{1}{e}}=(\pi+(z-\pi))^{\frac{1}{e}}=\pi_{L}\left(1+\frac{z-\pi}{\pi}\right)^{\frac{1}{e}}=\pi_{L}\left(1+\frac{1}{e} \frac{z-\pi}{\pi}+\frac{1}{2} \frac{1}{e}\left(\frac{1}{e}-1\right)\left(\frac{z-\pi}{\pi}\right)^{2}+\cdots\right) .
$$

Soit $D$ un $k((z))$-espace vectoriel. Pink fait remarquer dans [49] qu'en général, sans l'hypothèse $L / L_{0}$ séparable, à toute structure de Hodge-Pink $V$ (c'est-à-dire un $L[[z-\pi]]$-réseau dans $\left.{ }^{\tau} D \otimes_{k((z))} L((z-\pi))\right)$ on peut associer la structure de Hodge

$$
\mathrm{Fil}^{i}=\left((z-\pi)^{i} V \cap U_{D}\right) /\left((z-\pi)^{i} V \cap(z-\pi) U_{D}\right) .
$$

Cette application (qui à une structure de Hodge-Pink associe une structure de Hodge) est surjective mais pas injective. Lorsque $L / L_{0}$ est séparable on possède une section $\mathcal{T}$ de cette application, qui à une structure de Hodge $\left(\mathrm{Fil}^{i}\right)_{i \in \mathbb{Z}}$ (c'est-à-dire une filtration décroissante, séparée et exhaustive, de ${ }^{\tau} D \otimes_{k((z))} L$ par des sous- $L$-espaces vectoriels) associe la structure de Hodge-Pink $V$ définie par

$$
V=\sum_{i \in \mathbb{Z}}(z-\pi)^{-i} \mathrm{Fil}^{i} \otimes_{L, \iota} L[[z-\pi]] .
$$

Les opérateurs différentiels $k((z))$-linéaires sur $\oslash \widehat{\otimes} \vartheta_{L}\left[\frac{1}{z}\right]=k\left[\left[\pi_{L}\right]\right]((z))$ sont de la forme $\sum_{n \in \mathbb{Z}} a_{n} \frac{1}{n !}\left(\frac{d}{d \pi_{L}}\right)^{n}$ avec $a_{n} \in \Theta \widehat{\Theta} \theta_{L}\left[\frac{1}{z}\right]$, donc ils forment une $\Theta \widehat{\otimes} \theta_{L}\left[\frac{1}{z}\right]$-algèbre Diff engendrée par $\frac{d}{d \pi_{L}}, \frac{1}{p !}\left(\frac{d}{d \pi_{L}}\right)^{p}, \ldots$ Les éléments de Diff se prolongent par continuité en des 
opérateurs $k((z))$-linéaires sur $L[[z-\pi]]$ et $L((z-\pi))$. Ils agissent donc naturellement sur ${ }^{\tau} D \otimes_{k((z))} L[[z-\pi]]$.

Lemme 11.2. - On suppose $L / L_{0}$ séparable. Soit $V$ une structure de Hodge-Pink. Les assertions suivantes sont équivalentes

i) $V$ est dans l'image de la section $\mathcal{T}$,

ii) $V$ est stable par $(z-\pi) \frac{d}{d \pi_{L}},(z-\pi)^{p} \frac{1}{p !}\left(\frac{d}{d \pi_{L}}\right)^{p}, \ldots$ ou de façon équivalente $V$ est stable par $(z-\pi)^{\operatorname{deg} P} P$ pour tout $P \in$ Diff.

On dit que $V$ est tranquille lorsque ces assertions équivalentes sont satisfaites. La condition ii) fait penser à la transversalité de Griffiths.

Remarque. - D'après la condition (i), si $V$ est tranquille, pour tout $k \in \mathbb{Z},(z-\pi)^{k} V$ est tranquille.

Démonstration. - D'abord i) implique ii) car Diff agit trivialement sur $\iota(L)$. En effet par définition Diff agit trivialement sur $\iota\left(L_{0}\right)=k((z))$ et il suffit donc de montrer que $\iota\left(\pi_{L}\right)$ est tué par Diff. Soit $E \in L_{0}[t]$ le polynôme minimal de $\pi_{L}$ sur $L_{0}$. Comme $L / L_{0}$ est séparable $\frac{d E}{d t}\left(\iota\left(\pi_{L}\right)\right)$ est inversible dans $L[[z-\pi]]$. Comme $E\left(\iota\left(\pi_{L}\right)\right)=0$ on a $\left(\frac{d E}{d t}\left(\iota\left(\pi_{L}\right)\right)\right) \frac{d}{d \pi_{L}}\left(\iota\left(\pi_{L}\right)\right)=0$ d'où $\frac{d}{d \pi_{L}}\left(\iota\left(\pi_{L}\right)\right)=0$. On a alors

$$
0=\frac{1}{p !}\left(\frac{d}{d \pi_{L}}\right)^{p}\left(E\left(\iota\left(\pi_{L}\right)\right)\right)=\left(\frac{d E}{d t}\left(\iota\left(\pi_{L}\right)\right)\right) \frac{1}{p !}\left(\frac{d}{d \pi_{L}}\right)^{p}\left(\iota\left(\pi_{L}\right)\right)
$$

d'où $\frac{1}{p !}\left(\frac{d}{d \pi_{L}}\right)^{p}\left(\iota\left(\pi_{L}\right)\right)=0, \ldots$ Montrons que ii) implique i). Soit $V$ comme dans ii). On pose $U_{D}={ }^{\tau} D \otimes_{k((z))} L[[z-\pi]]$. Pour tout $i \in \mathbb{Z}$ on note

$$
\begin{aligned}
\mathrm{Fil}^{i} & =\left((z-\pi)^{i} V \cap U_{D}\right) /\left((z-\pi)^{i} V \cap(z-\pi) U_{D}\right) \\
& \subset U_{D} /(z-\pi) U_{D}={ }^{\tau} D \otimes_{k((z))} L .
\end{aligned}
$$

Il suffit de montrer l'inclusion $\sum_{i \in \mathbb{Z}}(z-\pi)^{-i} \mathrm{Fil}^{i} \otimes_{L, \iota} L[[z-\pi]] \subset V$. Cela résulte du fait que pour $i \in \mathbb{Z}, x \in{ }^{\tau} D \otimes_{k((z))} L$ et $y \in U_{D}$ dont la réduction modulo $(z-\pi) U_{D}$ est $x$, le $L[[z-\pi]]$-module engendré par $(z-\pi)^{i} y$ et ses images par les $(z-\pi)^{\operatorname{deg} P} P$ pour $P \in$ Diff contient $(z-\pi)^{i}(1 \otimes \iota)(x)$. Pour tout $i \in \mathbb{Z}$, la $L[[z-\pi]]$-algèbre engendrée par $(z-\pi){ }^{\operatorname{deg} P} P$ pour $P \in$ Diff est invariante par conjugaison par $(z-\pi)^{i}$ et il suffit donc de montrer l'assertion précédente pour $i=0$. On définit $\frac{d}{d \pi}=\left(\frac{d \pi}{d \pi_{L}}\right)^{-1} \frac{d}{d \pi_{L}}$ (on a $\frac{d \pi}{d \pi_{L}} \neq 0$ car

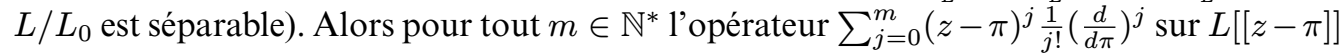
est $\iota(L)$-linéaire, envoie 1 sur 1 et annule $(z-\pi)^{j}$ pour $j \in\{1, \ldots, m\}$. Soient $m \in \mathbb{N}^{*}$ et $x_{1}, \ldots, x_{m} \in{ }^{\tau} D \otimes_{k((z))} L$ tels que $y=(1 \otimes \iota)(x)+\sum_{j=1}^{m}(z-\pi)^{j}(1 \otimes \iota)\left(x_{j}\right)$. Alors l'opérateur $\sum_{j=0}^{m}(z-\pi)^{j} \frac{1}{j !}\left(\frac{d}{d \pi}\right)^{j}$ envoie $y \operatorname{sur}(1 \otimes \iota)(x)$.

Supposons de nouveau $L / L_{0}$ séparable. La faible admissibilité pour une structure de Hodge-Pink tranquille équivaut à la faible admissibilité (au sens de Fontaine) pour la structure de Hodge $\left(\mathrm{Fil}^{i}\right)_{i \in \mathbb{Z}}$ associée, c'est-à-dire que pour tout sous-isochtouca $\left(D^{\prime}, \phi_{D^{\prime}}\right)$ de $\left(D, \phi_{D}\right)$, on a

$$
\sum_{i \in \mathbb{Z}} i \operatorname{dim}_{L}\left(\mathrm{Fil}^{i} \cap\left(D^{\prime} \otimes_{k((z))} L\right)\right) /\left(\mathrm{Fil}^{i+1} \cap\left(D^{\prime} \otimes_{k((z))} L\right)\right) \leq t_{N}\left(D^{\prime}\right)
$$


avec égalité pour $D^{\prime}=D$. Pour une structure de Hodge-Pink qui ne serait pas tranquille, la faible admissibilité au sens de Hodge-Pink est un énoncé plus faible que la faible admissibilité de la structure de Hodge associée (au sens de (80)).

Ainsi il y a une bijection entre les structures de Hodge-Pink tranquilles et les structures de Hodge usuelles. Il résulte de l'équivalence entre admissible et faiblement admissible (théorème 7.3) que les structures de Hodge-Pink faiblement admissibles forment une catégorie tannakienne (Pink l'a montré directement dans [49] si $D$ est isocline de pente 0 et sa démonstration s'adapte peut-être en général). Il en va de même pour les structures de HodgePink tranquilles faiblement admissibles, donc pour les structures de Hodge faiblement admissibles. L'hypothèse que $L / L_{0}$ est séparable est vraiment nécessaire pour relever les structures de Hodge faiblement admissibles en des structures de Hodge-Pink faiblement admissibles car on peut adapter le contre-exemple 5.16 de [49] pour montrer que les structures de Hodge faiblement admissibles (au sens de (80)) ne forment pas en général une catégorie tannakienne, si $L / L_{0}$ n'est pas séparable. En effet on prend $q=2, L_{0}=\mathbb{F}_{2}((\pi)), L=\mathbb{F}_{2}((\sqrt{\pi}))$, $D=\left(\mathbb{F}_{2}((\pi))\right)^{2}, \phi_{D}$ de matrice identité, $\mathrm{Fil}^{-1}={ }^{\tau} D \otimes_{L_{0}} L, \mathrm{Fil}^{0}=\mathrm{Fil}^{1}$ égal à la droite engendrée par $\left(\begin{array}{c}1 \\ \sqrt{\pi}\end{array}\right) \in{ }^{\tau} D \otimes_{L_{0}} L$ et $\mathrm{Fil}^{2}=0$, de sorte que les pentes de Hodge sont -1 et 1 (et on a $t_{H}=t_{N}=0$ ). Alors $D$ est faiblement admissible mais pas $D \otimes D$, à cause du sous-isochtouca de $D \otimes D$ engendré par

$$
w_{1}=\left(\begin{array}{l}
1 \\
0
\end{array}\right) \otimes\left(\begin{array}{l}
0 \\
1
\end{array}\right)+\left(\begin{array}{l}
0 \\
1
\end{array}\right) \otimes\left(\begin{array}{l}
1 \\
0
\end{array}\right) \text { et } w_{2}=\left(\begin{array}{l}
1 \\
0
\end{array}\right) \otimes\left(\begin{array}{l}
1 \\
0
\end{array}\right)+\pi\left(\begin{array}{l}
0 \\
1
\end{array}\right) \otimes\left(\begin{array}{l}
0 \\
1
\end{array}\right)
$$

pour lequel $t_{N}$ est nul mais dont les pentes de Hodge sont 0 et 2, puisque

$$
\sqrt{\pi} w_{1}+w_{2}=\left(\begin{array}{c}
1 \\
\sqrt{\pi}
\end{array}\right) \otimes\left(\begin{array}{c}
1 \\
\sqrt{\pi}
\end{array}\right) \text { et } w_{1}=\left(\begin{array}{c}
1 \\
\sqrt{\pi}
\end{array}\right) \otimes\left(\begin{array}{l}
0 \\
1
\end{array}\right)+\left(\begin{array}{l}
0 \\
1
\end{array}\right) \otimes\left(\begin{array}{c}
1 \\
\sqrt{\pi}
\end{array}\right) .
$$

On peut se demander quels chtoucas et isochtoucas locaux donnent des structures de Hodge-Pink tranquilles.

Lemme 11.3. - Soit $N$ un isochtouca local sur $\emptyset_{L}$. Alors la structure de Hodge-Pink associée est tranquille si et seulement si, pour tout entier $n \in \mathbb{N}^{*}$,

$$
(z-\pi)^{n}\left(\phi_{N}{ }^{\tau} \phi_{N} \tau^{2} \phi_{N} \cdots\right) \frac{1}{n !}\left(\frac{d}{d \pi_{L}}\right)^{n}\left(\phi_{N}{ }^{\tau} \phi_{N} \tau^{2} \phi_{N} \cdots\right)^{-1}
$$

n'a pas de pôle en $z=\pi$.

On dit alors que l'isochtouca local est tranquille.

Pour donner un sens à la condition du lemme, choisissons une base de $N$ sur $\theta \widehat{\otimes} \theta_{L}\left[\frac{1}{z}\right]$, de sorte que $\phi_{N}$ est une matrice à coefficients dans $\theta \widehat{\otimes} \theta_{L}\left[\frac{1}{z}, \frac{1}{z-\pi}\right]$. Si $k$ est un entier tel que $n<q^{k}$, l'expression ci-dessus est définie comme étant

$$
(z-\pi)^{n}\left(\phi_{N}{ }^{\tau} \phi_{N} \ldots \tau^{k-1} \phi_{N}\right) \frac{1}{n !}\left(\frac{d}{d \pi_{L}}\right)^{n}\left(\phi_{N}{ }^{\tau} \phi_{N} \ldots \tau^{k-1} \phi_{N}\right)^{-1}
$$

et c'est une matrice à coefficients dans $\oslash \widehat{\otimes} \oslash_{L}\left[\frac{1}{z}, \frac{1}{z-\pi}, \ldots, \frac{1}{z-\pi^{q^{k-1}}}\right]$. 
Démonstration du lemme 11.3. - Comme les opérateurs différentiels $\frac{1}{n !}\left(\frac{d}{d \pi_{L}}\right)^{n}$ sont $k((z))$-linéaires, ils commutent à $\phi_{D},{ }^{\tau} \phi_{D}, \ldots$ et donc la condition du lemme équivaut à : pour tout $n \in \mathbb{N}^{*},(z-\pi)^{n} R^{-1} \frac{1}{n !}\left(\frac{d}{d \pi_{L}}\right)^{n} R$ n'a pas de pôle en $z=\pi$. On voit tout de suite que cela équivaut à ii) du lemme 11.2.

Pour tout élément $x$ d'un localisé de $\oslash \widehat{\otimes} \vartheta_{L}$, on a $\frac{1}{n !}\left(\frac{d}{d \pi_{L}}\right)^{n}\left({ }^{\tau} x\right)=0$ si $q$ ne divise pas $n$ et $\frac{1}{n !}\left(\frac{d}{d \pi_{L}}\right)^{n}\left({ }^{\tau} x\right)={ }^{\tau}\left(\frac{1}{(n / q) !}\left(\frac{d}{d \pi_{L}}\right)^{(n / q)}(x)\right)$ si $q$ divise $n$.

On en déduit que si $N$ est un isochtouca local tranquille, pour tout $n \in \mathbb{N}^{*}$, $\left(\phi_{N}{ }^{\tau} \phi_{N} \tau^{2} \phi_{N} \cdots\right) \frac{1}{n !}\left(\frac{d}{d \pi_{L}}\right)^{n}\left(\phi_{N}{ }^{\tau} \phi_{N} \tau^{2} \phi_{N} \cdots\right)^{-1}$ appartient en fait à

$$
(z-\pi)^{-n}\left(z-\pi^{q}\right)^{-[n / q]}\left(z-\pi^{q^{2}}\right)^{-\left[n / q^{2}\right]} \cdots \operatorname{Hom}_{\vartheta \widehat{\otimes} \emptyset_{L}\left[\frac{1}{z}\right]}(N, N) .
$$

On dit qu'un chtouca local sur $\theta_{L}$ est tranquille si l'isochtouca local associé est tranquille.

Lemme 11.4. - Un chtouca local $M=\left(M, \phi_{M}\right)$ sur $\theta_{L}$ est tranquille si et seulement si pour tout $n \in \mathbb{N}^{*},\left(\phi_{M}{ }^{\tau} \phi_{M} \tau^{2} \phi_{M} \cdots\right) \frac{1}{n !}\left(\frac{d}{d \pi_{L}}\right)^{n}\left(\phi_{M}{ }^{\tau} \phi_{M}{ }^{\tau^{2}} \phi_{M} \cdots\right)^{-1}$ appartient $\grave{a}$

$$
(z-\pi)^{-n}\left(z-\pi^{q}\right)^{-[n / q]}\left(z-\pi^{q^{2}}\right)^{-\left[n / q^{2}\right]} \cdots \operatorname{Hom}_{\partial \widehat{\otimes} \vartheta_{L}}(M, M) .
$$

Grâce à ce lemme on va associer à un chtouca local tranquille sur $\theta_{L}$ un « $\theta$-cristal» avec une notion de puissances divisées au sens de Grothendieck et Berthelot, comme dans la définition 6.1. Cela indique que pour les chtoucas locaux tranquilles les puissances divisées nécessaires sont les mêmes que celles qui apparaissent en inégales caractéristiques. On a vu dans le paragraphe 4 que pour les chtoucas locaux non minuscules cela n'est pas vrai sans l'hypothèse de tranquillité.

Nous abandonnons à partir de maintenant la notion de $\theta$-cristal de la définition 5.3, qui utilisait les puissances divisées au sens de Honda et Gross-Hopkins introduites dans la définition 5.1 et qui n'était adaptée qu'au cas minuscule. Dorénavant nous utilisons les puissances divisées au sens de Grothendieck et Berthelot, introduites dans la définition 6.1.

Soit $\&$ le complété $z$-adique de $\oslash \widehat{\otimes} \vartheta_{L}\left[\frac{\pi^{q}}{z}, \frac{\pi^{q^{2}}}{z^{q+1}}, \frac{\pi^{q^{3}}}{z^{q^{2}+q+1}}, \ldots\right]$. En utilisant les coordonnées $(u, \pi)$ de $\phi_{\text {min }}$ venant de l'isomorphisme $\phi_{\text {min }}=\hat{B}$ de la proposition 10.2 , on verra aussi $\&$ comme le complété pour la topologie $\pi$-adique de $(\theta \widehat{\otimes} k)[[u]]\left[\frac{u^{e q}}{\pi}, \frac{u^{e q^{2}}}{\pi^{q+1}}, \ldots\right]$. C'est une enveloppe à puissances divisées au sens de Grothendieck et Berthelot de $(\theta \widehat{\otimes} k)[[u]]$ (alors que $\&_{\min }$ était une enveloppe à puissances divisées au sens de Honda et Gross-Hopkins de $(\theta \widehat{\otimes} k)[[u]])$. Donc $\&$ est vraiment l'analogue de l'anneau $\&$ qui apparaît dans [14] sous ce nom et qui est noté $R_{V}$ dans [20].

En utilisant la formule de Taylor et en procédant comme dans le paragraphe IV.1.6 de [4], on peut montrer que la catégorie des $\theta$-cristaux relativement à $\left(\theta, \pi \theta, \gamma_{\vartheta}\right)$ sur $\theta_{L}$ (ou, de façon équivalente, grâce à l'analogue du lemme 5.5 pour les puissances divisées au sens de Grothendieck et Berthelot, sur $\theta_{L} / \pi \theta_{L}$ ) est équivalente à la catégorie des $\ell$-modules libres munis d'une " connexion » par rapport aux opérateurs différentiels remultipliés $z^{[n / q]+\left[n / q^{2}\right]+\left[n / q^{3}\right]+\cdots} \frac{1}{n !}\left(\frac{d}{d \pi_{L}}\right)^{n}$ et c'est ce point de vue qui nous servira dans la suite.

Le lemme précédent montre que si $M$ est un chtouca local tranquille, $\mathcal{M}={ }^{\tau} M \otimes \&$ est muni d'une connexion par rapport aux opérateurs différentiels remultipliés 
$z^{[n / q]+\left[n / q^{2}\right]+\left[n / q^{3}\right]+\cdots} \frac{1}{n !}\left(\frac{d}{d \pi_{L}}\right)^{n}$. Plus précisément l'action d'un tel opérateur est donnée par la formule

$$
z^{[n / q]+\left[n / q^{2}\right]+\left[n / q^{3}\right]+\cdots}\left({ }^{\tau} \phi_{M} \tau^{2} \phi_{M} \cdots\right) \frac{1}{n !}\left(\frac{d}{d \pi_{L}}\right)^{n}\left({ }^{\tau} \phi_{M}{ }^{\tau^{2}} \phi_{M} \cdots\right)^{-1}
$$

et cette formule a un sens car $z^{[n / q]+\left[n / q^{2}\right]+\left[n / q^{3}\right]+\cdots}\left(z-\pi^{q}\right)^{-[n / q]}\left(z-\pi^{q^{2}}\right)^{-\left[n / q^{2}\right]} \cdots$ est une unité de $\phi$. Cette formule vient du fait que l'action doit être compatible avec l'inclusion $\mathcal{M} \subset{ }^{\tau} D \otimes_{k((z))} \&\left[\frac{1}{z}\right]$, et que l'action de ces opérateurs sur ${ }^{\tau} D \otimes_{k((z))} \&\left[\frac{1}{z}\right]$ découle de leur $k((z))$-linéarité (i.e. ils agissent trivialement $\operatorname{sur}^{\tau} D$ ).

Le point le plus subtil est le fait que $\mathcal{M}$ ne dépend que de la réduction de $M$ modulo $\pi$. Mais comment exprimer que deux chtoucas locaux tranquilles sont égaux modulo $\pi$ ? Un simple isomorphisme entre les réductions modulo $\pi$ (en oubliant la tranquillité) ne suffit pas, comme le montre l'exemple suivant.

Exemple 11.5. - Soient $m \in\{1, \ldots, q-1\}, y \in \Theta \widehat{\otimes} \widehat{\theta}_{L}$ et $\left(M, \phi_{M}\right)$ le chtouca local d'amplitude $\subset[0, m]$ défini par $M=\left(\Theta \widehat{\otimes} \vartheta_{L}\right)^{2}$ et $\phi_{M}=\left(\begin{array}{cc}(z-\pi)^{m} & 0 \\ y & 1\end{array}\right)$. Alors $\left(M, \phi_{M}\right)$ est tranquille si et seulement si pour tout $i \in\{1, \ldots, m-1\}$ on a $\frac{1}{i !}\left(\frac{d}{d \pi_{L}}\right)^{i} y \in(z-\pi)^{m-i} \oslash \widehat{\otimes} \widehat{\vartheta}_{L}$. Ces conditions sont vérifiées si l'on prend $y=\pi_{L}^{q a}$ avec $a \in \mathbb{N}$. Supposons e $\leq q a<e m$. Soient $\left(M^{\prime}, \phi_{M^{\prime}}\right)$ le chtouca défini par $M^{\prime}=\left(\Theta \widehat{\otimes} \theta_{L}\right)^{2}$ et $\phi_{M^{\prime}}=\left(\begin{array}{cr}(z-\pi)^{m} & 0 \\ 0 & 1\end{array}\right)$. Alors $\left(M, \phi_{M}\right)$ et $\left(M^{\prime}, \phi_{M^{\prime}}\right)$ sont congrus modulo $\pi$ et pourtant ${ }^{\tau}\left(R^{\prime-1} R\right) \notin G L_{2}(\varnothing)$.

Nous dirons que deux chtoucas locaux tranquilles $M_{1}$ et $M_{2} \operatorname{sur} \theta_{L}$ sont égaux modulo $\pi$ s'il existe un chtouca local tranquille sur $\theta_{L}[[x]]$ dont ils sont les évaluations en $x=0$ et $x=\pi$. Le point remarquable est qu'alors on a un isomorphisme canonique entre les $\&$-modules $\mathcal{M}_{1}$ et $\mathcal{M}_{2}$ associés à $M_{1}$ et $M_{2}$ ! Commençons par définir ce qu'est un chtouca local tranquille sur une base plus générale que $\theta_{L}$.

La définition suivante est provisoire. Elle est certainement la bonne dans le cas que nous avons en vue $\left(S=\operatorname{Spf} \theta_{L}[[x]]\right)$.

DÉfinition 11.6. - On dit qu'un chtouca local $\left(M, \phi_{M}\right)$ sur un schéma formel régulier $S$ sur $\operatorname{Spf} \Theta$ est tranquille si, localement pour la topologie de Zariski sur $S$, pour tout opérateur différentiel $P$, continu de degré $\leq n$, sur $S$ (étendu par $\theta$-linéarité à $\Theta \widehat{\otimes} \vartheta_{S}$ ),

$$
\begin{aligned}
& \left(\phi_{M}{ }^{\tau} \phi_{M} \tau^{2} \phi_{M} \cdots\right) P\left(\phi_{M}{ }^{\tau} \phi_{M} \tau^{2} \phi_{M} \cdots\right)^{-1} \text { appartient } \grave{a} \\
& (z-\pi)^{-n}\left(z-\pi^{q}\right)^{-[n / q]}\left(z-\pi^{q^{2}}\right)^{-\left[n / q^{2}\right]} \cdots \operatorname{Hom}_{\vartheta}(M, M) .
\end{aligned}
$$

Précisons que pour tout $k \in \mathbb{N}$ tel que $q^{k}>n$ on définit

$$
\left(\phi_{M}{ }^{\tau} \phi_{M} \tau^{2} \phi_{M} \cdots\right) P\left(\phi_{M}{ }^{\tau} \phi_{M} \tau^{2} \phi_{M} \cdots\right)^{-1}=\left(\phi_{M}{ }^{\tau} \phi_{M} \cdots \tau^{k-1} \phi_{M}\right) P\left(\phi_{M}{ }^{\tau} \phi_{M} \cdots \tau^{k-1} \phi_{M}\right)^{-1},
$$

et que $P$ agit sur $\theta \widehat{\otimes} \theta_{S}$ en envoyant $a \otimes b \in \Theta \widehat{\otimes} \theta_{S}$ sur $a \otimes P(b)$ et agit trivialement sur $\tau^{k} x$ pour $x \in M$. 
ReMARQUe. - La condition (81) implique une condition apparemment plus forte, à savoir que pour tout $Q$ dans la sous- $\Theta \widehat{\otimes} \vartheta_{S}$-algèbre de l'algèbre des opérateurs différentiels continus $\theta$-linéaires sur $\theta \widehat{\otimes} \theta_{S}$ engendrée par les

$$
(z-\pi)^{n}\left(z-\pi^{q}\right)^{[n / q]}\left(z-\pi^{q^{2}}\right)^{\left[n / q^{2}\right]} \ldots P
$$

avec $P$ de degré $\leq n$, on a $\left(\phi_{M}{ }^{\tau} \phi_{M} \tau^{2} \phi_{M} \cdots\right) Q\left(\phi_{M}{ }^{\tau} \phi_{M} \tau^{2} \phi_{M} \cdots\right)^{-1} \in \operatorname{Hom}_{\vartheta}(M, M)$.

On peut étendre la définition précédente au cas d'une base $S$ non nécessairement régulière en considérant des opérateurs différentiels à valeurs dans des modules sur $\theta_{S}$ (voir [27] et EGA IV 16). Il suffit de considérer les opérateurs différentiels universels à valeurs dans des espaces de jets et la définition obtenue se reformule de la façon suivante. De façon plus élémentaire, grâce à la formule de Taylor et à la remarque précédente, la définition 11.7 généralise la définition 11.6.

DéFINITION 11.7. - On dit qu'un chtouca local $\left(M, \phi_{M}\right)$ sur un schéma formel $S$ sur $\operatorname{Spf} \theta$ est tranquille si pour tout $n \in \mathbb{N}^{*}$, en notant $\Delta$ la diagonale du schéma formel $S \widehat{\times}_{\operatorname{Spec} \mathbb{F}_{q}} S$, $I_{\Delta}$ son idéal, et $\Delta^{\left(q^{n}\right)}$ le fermé d'idéal $I_{\Delta}^{\left(q^{n}\right)}$, et $p_{1}, p_{2}: I_{\Delta}^{\left(q^{n}\right)} \rightarrow S$ les deux projections, le morphisme de $\Theta \widehat{\otimes} \vartheta_{\Delta^{\left(q^{n}\right)}}\left[\frac{1}{z-p_{1}^{*}(\pi)}, \ldots, \frac{1}{z-p_{1}^{*}\left(\pi^{q^{n-1}}\right)}\right]$-modules

$$
p_{2}^{*}\left(\phi_{M}\right) p_{2}^{*}\left({ }^{\tau} \phi_{M}\right) \cdots p_{2}^{*}\left(\tau^{n-1} \phi_{M}\right) p_{1}^{*}\left(\tau^{n-1} \phi_{M}\right)^{-1} \cdots p_{1}^{*}\left({ }^{\tau} \phi_{M}\right)^{-1} p_{1}^{*}\left(\phi_{M}\right)^{-1}
$$

appartient $\grave{a}$

$$
\operatorname{Hom}_{\partial \widehat{\otimes} \vartheta_{\Delta^{\left(q^{n}\right)}}}\left(p_{1}^{*}(M), p_{2}^{*}(M)\right) \otimes_{\partial \widehat{\otimes}_{\vartheta_{\Delta}^{\left(q^{n}\right)}}} \mathcal{T}_{n}
$$

où l'on désigne par $\mathcal{T}_{n}$ la sous- $\Theta \widehat{\otimes} \vartheta_{\Delta^{\left(q^{n}\right)}}$-algèbre de

$$
\theta \widehat{\otimes} \theta_{\Delta\left(q^{n}\right)}\left[\frac{1}{z-p_{1}^{*}(\pi)}, \ldots, \frac{1}{z-p_{1}^{*}\left(\pi^{q^{n-1}}\right)}\right]
$$

engendrée par

$\frac{I_{\Delta}}{z-p_{1}^{*}(\pi)}, \frac{I_{\Delta}^{(q)}}{\left(z-p_{1}^{*}(\pi)\right)^{q}\left(z-p_{1}^{*}\left(\pi^{q}\right)\right)}, \ldots, \frac{I_{\Delta}^{\left(q^{n-1}\right)}}{\left(z-p_{1}^{*}(\pi)\right)^{q^{n-1}}\left(z-p_{1}^{*}\left(\pi^{q}\right)\right)^{q^{n-2}} \cdots\left(z-p_{1}^{*}\left(\pi^{q^{n-1}}\right)\right)}$.

On a noté $p_{i}^{*}(M)=M \otimes_{\vartheta \widehat{\otimes} \vartheta_{S}, 1 \widehat{\otimes} p_{i}^{*}} \oslash \widehat{\otimes} \vartheta_{\Delta^{\left(q^{n}\right)}}$ et on a utilisé implicitement l'isomorphisme $p_{1}^{*}\left(\tau^{n} M\right) \simeq p_{2}^{*}\left(\tau^{n} M\right)$ qui est l'image inverse de Id par le morphisme $\sigma^{n}: \Delta^{\left(q^{n}\right)} \rightarrow \Delta$, où $\sigma$ désigne le morphisme de Frobenius de $S \times S$ dans lui-même qui à une section locale $x$ du faisceau structural associe $x^{q}$. D'autre part on note que la condition pour $n$ implique la condition pour $n-1$.

Remarque. - La définition précédente est provisoire. Sur des bases générales la tranquillité s'exprime sans doute par une structure additionnelle que nous n'avons pas encore su dégager. En particulier l'exemple avant la définition 11.6 et le lemme 11.8 montrent que nous n'avons pas la bonne définition d'un chtouca local tranquille sur $\theta_{L} / \pi \theta_{L}$.

Le lemme suivant justifie le fait que si $M$ est un chtouca local tranquille sur $\theta_{L}$, $\mathcal{M}={ }^{\tau} M \otimes \varnothing$ ne dépend « que de la réduction de $M$ modulo $\pi »$. 
Lemme 11.8. - Soient $m \in \mathbb{N}$ et $M=\left(M, \phi_{M}\right)$ un chtouca local tranquille (au sens de la définition 11.7) sur $S=\operatorname{Spf}\left(\theta_{L}[[x]]\right)$ d'amplitude $\subset[0, m]$. Soient $M_{1}$ et $M_{2}$ les chtoucas locaux tranquilles sur $\theta_{L}$ obtenus en faisant $x=0$, resp. $x=\pi$. Soit $\left(D, \phi_{D}\right)$ la réduction de $M$ modulo $\left(x, \pi_{L}\right)$ (qui est donc aussi la réduction de $M_{1}$ ou $M_{2}$ modulo $\pi_{L}$ ). Alors $\mathcal{M}_{1}=\mathcal{M}_{2}$ dans $^{\tau} D \otimes \varnothing\left[\frac{1}{z}\right]$.

Démonstration. - On choisit une base de $M$ (d'où des bases de $M_{1}, M_{2}$ et $D$ ). Soit $n \in \mathbb{N}^{*}$. En se restreignant dans la définition 11.7 au sous-schéma formel $S \widehat{\times}_{\mathrm{Spf} \vartheta_{L}} S$ de $S \widehat{\times}_{\operatorname{Spec} \mathbb{F}} S$, en notant, pour $i=1,2, p_{i}: \operatorname{Spf}\left(\theta_{L}\left[\left[x_{1}, x_{2}\right]\right]\right) \rightarrow \operatorname{Spf}\left(\theta_{L}[[x]]\right)$ le morphisme tel que $p_{i}^{*}$ soit l'identité sur $\vartheta_{L}$ et $p_{i}^{*}(x)=x_{i}$, et en appliquant la factorialité de $\theta \widehat{\otimes} \Theta_{L}\left[\left[x_{1}\right]\right]$ aux coefficients du développement en puissances de $x_{1}-x_{2}$ de l'expression

$$
p_{2}^{*}\left(\phi_{M}\right) p_{2}^{*}\left({ }^{\tau} \phi_{M}\right) \cdots p_{2}^{*}\left(\tau^{n-1} \phi_{M}\right) p_{1}^{*}\left(\tau^{n-1} \phi_{M}\right)^{-1} \cdots p_{1}^{*}\left({ }^{\tau} \phi_{M}\right)^{-1} p_{1}^{*}\left(\phi_{M}\right)^{-1}
$$

on obtient que modulo $\left(x_{1}-x_{2}\right)^{q^{n}}$ celle-ci appartient à

$$
\begin{aligned}
M_{r}\left(( \ominus \widehat { \otimes } \vartheta _ { L } [ [ x _ { 1 } , x _ { 2 } ] ] / ( x _ { 1 } - x _ { 2 } ) ^ { q ^ { n } } ) \left[\frac{x_{1}-x_{2}}{z-\pi}, \frac{\left(x_{1}-x_{2}\right)^{q}}{(z-\pi)^{\min (m, q)}\left(z-\pi^{q}\right)}, \ldots,\right.\right. \\
\left.\left.\frac{\left(x_{1}-x_{2}\right)^{q^{n-1}}}{(z-\pi)^{\min \left(m, q^{n-1}\right.}\left(z-\pi^{q}\right)^{\min \left(m, q^{n-2}\right)} \cdots\left(z-\pi^{q^{n-1}}\right)}\right]\right),
\end{aligned}
$$

d'où

$$
\begin{gathered}
p_{2}^{*}\left(\phi_{M}\right) p_{2}^{*}\left({ }^{\tau} \phi_{M}\right) \cdots p_{2}^{*}\left(\tau^{n-1} \phi_{M}\right) p_{1}^{*}\left(\tau^{n-1} \phi_{M}\right)^{-1} \cdots p_{1}^{*}\left({ }^{\tau} \phi_{M}\right)^{-1} p_{1}^{*}\left(\phi_{M}\right)^{-1} \\
\in M_{r}\left(\Theta \widehat { \otimes } \theta _ { L } [ [ x _ { 1 } , x _ { 2 } ] ] \left[\frac{x_{1}-x_{2}}{z-\pi}, \frac{\left(x_{1}-x_{2}\right)^{q}}{(z-\pi)^{\min (m, q)}\left(z-\pi^{q}\right)}, \ldots,\right.\right. \\
\frac{\left(x_{1}-x_{2}\right)^{q^{n-1}}}{(z-\pi)^{\min \left(m, q^{n-1}\right)}\left(z-\pi^{q}\right)^{\min \left(m, q^{n-2}\right)} \cdots\left(z-\pi^{q^{n-1}}\right)}, \\
\left.\left.\frac{\left(x_{1}-x_{2}\right)^{q^{n}}}{(z-\pi)^{m}\left(z-\pi^{q}\right)^{m} \cdots\left(z-\pi^{q^{n-1}}\right)^{m}}\right]\right) .
\end{gathered}
$$

En prenant $x_{1}=0$ et $x_{2}=\pi$, on en déduit que pour tout $n \in \mathbb{N}^{*}$,

$$
\begin{array}{r}
{ }^{\tau} \phi_{M_{2}} \ldots{ }^{\tau^{n}} \phi_{M_{2}}{ }^{\tau^{n}} \phi_{M_{1}}^{-1} \cdots^{\tau} \phi_{M_{1}}^{-1} \in M_{r}\left(\oslash \widehat { \otimes } \oslash _ { L } \left[\frac{\pi^{q}}{z-\pi^{q}}, \frac{\pi^{q^{2}}}{\left(z-\pi^{q}\right)^{\min (m, q)}\left(z-\pi^{q^{2}}\right)},\right.\right. \\
\cdots, \frac{\pi^{q^{n}}}{\left(z-\pi^{q}\right)^{\min \left(m, q^{n-1}\right)}\left(z-\pi^{q^{2}}\right)^{\min \left(m, q^{n-2}\right) \cdots\left(z-\pi^{q^{n}}\right)}}, \\
\left.\left.\frac{\pi^{q^{n+1}}}{\left(z-\pi^{q}\right)^{m}\left(z-\pi^{q^{2}}\right)^{m} \cdots\left(z-\pi^{q^{n}}\right)^{m}}\right]\right) .
\end{array}
$$

Pour $n$ assez grand $\left(1+q+\cdots+q^{n}\right)-m n \geq 0$ donc cet élément appartient à $M_{r}(\phi)$. Comme $\&$ est complet pour la topologie $z$-adique et que le morphisme $\mathscr{C} \rightarrow \&\left[\frac{1}{z}\right]$ est continu, $\lim _{n \mapsto+\infty}{ }^{\tau} \phi_{M_{2}} \ldots \tau^{n} \phi_{M_{2}} \tau^{n} \phi_{M_{1}}^{-1} \ldots{ }^{\tau} \phi_{M_{1}}^{-1}$ appartient à $M_{r}(\phi)$.

La remarque suivante sera utile à la fin du paragraphe.

Remarque. - Si $e=1$, et si $\left(M, \phi_{M}\right)$ est un chtouca local tranquille sur $\theta_{L}$, on a $M={ }^{\tau} \Delta \otimes_{k[[z]]} \&$ dans ${ }^{\tau} M \otimes_{\vartheta \widehat{\otimes} \vartheta_{L}} \&\left[\frac{1}{z}\right]={ }^{\tau} D \otimes_{k((z))} \phi\left[\frac{1}{z}\right]$. On le montre en remplaçant $\vartheta_{L}\left[\left[x_{1}, x_{2}\right]\right]$ par $\vartheta_{L} \widehat{\otimes}_{\mathbb{F}_{q}} \vartheta_{L}$ et $x_{1}$ et $x_{2}$ par $\pi \otimes 1$ et $1 \otimes \pi$ dans la démonstration du lemme 11.8 
(c'est-à-dire qu'on applique la définition 11.7 à $S \widehat{\times}_{\text {Spec } \mathbb{F}_{q}} S$ sans se restreindre à $S \widehat{\times}_{\operatorname{Spf}} S$, en prenant $S=\operatorname{Spf} \theta_{L}$ au lieu de $\left.S=\operatorname{Spf}\left(\theta_{L}[[x]]\right)\right)$ puis en quotientant par $1 \otimes \pi$ dans $\theta_{L} \widehat{\otimes}_{\mathbb{F}_{q}} \theta_{L}$.

Voici quelques considérations générales relatives au lien entre la condition de tranquillité et la transversalité de Griffiths. Soient $k$ un corps parfait contenant $\mathbb{F}_{q}$ et $B$ une $\theta \widehat{\otimes} k$-algèbre régulière complète pour la topologie $\pi$-adique et sans $\pi$-torsion. Soit $\left(D, \phi_{D}\right)$ un isochtouca local sur $k$. Soit $\left(N, \phi_{N}\right)$ un pseudo-isochtouca local sur le Spf $\theta$-schéma formel Spf $B$. Soit $\rho$ une rigidification, c'est-à-dire un isomorphisme entre la réduction modulo $\pi$ de $\left(N, \phi_{N}\right)$ et l'image inverse de $\left(D, \phi_{D}\right)$ à $B / \pi B$. D'après le paragraphe 3 on associe à $\left(N, \phi_{N}\right)$ et $\rho$ une structure de Hodge-Pink, c'est-à-dire un $B\left[\frac{1}{\pi}\right][[z-\pi]]$-réseau $V$ dans ${ }^{\tau} D \otimes_{k((z))} B\left[\frac{1}{\pi}\right]((z-\pi))$. Supposons que

(T) pour tout opérateur différentiel $P$ continu de degré $\leq n$ sur $B$, que l'on étend à $B\left[\frac{1}{\pi}\right][[z-\pi]]$ par $k((z))$-linéarité et continuité, on a $(z-\pi)^{n} P V \subset V$.

En utilisant la platitude de $B \operatorname{sur} \theta$ et la factorialité de $\theta \widehat{\otimes} \theta$ on peut montrer que si $\left(N, \phi_{N}\right)$ provient d'un chtouca local $\left(M, \phi_{M}\right)$ sur $\operatorname{Spf} B$, la condition (T) est réalisée si et seulement si $\left(M, \phi_{M}\right)$ est tranquille au sens de la définition 11.6. Quitte à localiser supposons que $N$ est un $\oslash \widehat{\otimes} B\left[\frac{1}{z}\right]$-module libre et fixons une base de $D$ sur $k((z))$. Alors $V$ est un élément de

$$
G L_{r}\left(B\left[\frac{1}{\pi}\right]((z-\pi))\right) / G L_{r}\left(B\left[\frac{1}{\pi}\right][[z-\pi]]\right)
$$

et on dit que les nombres de Hodge sont constants s'il appartient à

$$
G L_{r}\left(B\left[\frac{1}{\pi}\right][[z-\pi]]\right)\left(\begin{array}{cccc}
(z-\pi)^{h_{1}} & 0 & \cdots & 0 \\
0 & (z-\pi)^{h_{2}} & \ddots & \vdots \\
\vdots & \ddots & \ddots & 0 \\
0 & \cdots & 0 & (z-\pi)^{h_{r}}
\end{array}\right) G L_{r}\left(B\left[\frac{1}{\pi}\right][[z-\pi]]\right)
$$

pour certains entiers $h_{1}, \ldots, h_{r} \in \mathbb{Z}$. Supposons que les nombres de Hodge sont constants. Alors on peut associer à $V$ une filtration décroissante, dite de Hodge, de ${ }^{\tau} D \otimes_{\mathbb{F}_{q}((z))} B\left[\frac{1}{\pi}\right]$ par des sous- $B\left[\frac{1}{\pi}\right]$-modules facteurs directs, en posant $U_{D}={ }^{\tau} D \otimes_{\mathbb{F}_{q}((z))} B\left[\frac{1}{\pi}\right][[z-\pi]]$ et

$$
\mathrm{Fil}^{i}=\left((z-\pi)^{i} V \cap U_{D}\right) /\left((z-\pi)^{i} V \cap(z-\pi) U_{D}\right) .
$$

Sous la condition $(\mathrm{T})$, cette filtration vérifie la transversalité de Griffiths. Plus précisément, pour tout opérateur différentiel $\theta$-linéaire continu $P$ sur $B$, de degré $\leq n$ (que l'on étend à $B\left[\frac{1}{\pi}\right]$ ), et pour $i \in \mathbb{Z}$ et $x \in \mathrm{Fil}^{i}$ on a $P(x) \in \mathrm{Fil}^{i-n}$. Il est intéressant de noter que cette condition de transversalité de Griffiths de la structure de Hodge associée n'a de sens que pour les opérateurs différentiels $\oslash$-linéaires sur $B$ (pour qu'elle ne dépende pas du choix de la base de $D$ ) alors que la condition (T) s'exprime en termes d'opérateurs différentiels non nécessairement $\theta$-linéaires sur $B$.

À partir de maintenant on suppose $m \leq q-2$. On suppose toujours $L / L_{0}$ séparable. Soit $C_{m, \text { tran }}$ la catégorie des chtoucas locaux tranquilles $\left(M, \phi_{M}\right)$ sur $\theta_{L}$ tels que $\phi_{M}$ et $(z-\pi)^{m} \phi_{M}^{-1}$ n'aient pas de pôle en $z=\pi$ (c'est-à-dire d'amplitude $\subset[0, m]$ ). Soit $C_{m \text {,tran }}^{\prime}$ la catégorie des $\left(D, \phi_{D}, V_{D}, \mathcal{M}\right)$ où 
- $\left(D, \phi_{D}\right)$ est un isochtouca,

- $V_{D}$ est une structure de Hodge-Pink tranquille telle que $U_{D} \subset V_{D} \subset(z-\pi)^{-m} U_{D}$,

- $\mathcal{M}$ est un $\&$-module libre, muni d'un isomorphisme $\mathcal{M} \otimes_{\&} \&\left[\frac{1}{z}\right] \simeq{ }^{\tau} D \otimes_{k((z))} \&\left[\frac{1}{z}\right]$,

- on suppose que $\mathcal{M}$ est « fortement divisible», c'est-à-dire

$$
\text { \&.z } z^{-m \tau}\left(\left(\phi_{D} \otimes 1\right)\left(\mathcal{M} \cap(z-\pi)^{m} V_{D}\right)\right)=\mathcal{M}
$$

Comme $\mathcal{M} \cap(z-\pi)^{m} V_{D} \supset(z-\pi)^{m} \mathcal{M}$ et $1-\frac{\pi^{q}}{z}$ est une unité dans $\&$, l'hypothèse que $\mathcal{M}$ est «fortement divisible» implique qu'il est stable par $(1 \widehat{\otimes} \mathrm{Fr}) \circ\left(\phi_{D} \otimes 1\right)$ (condition demandée par Breuil dans [14]).

On note $\mathbb{D}^{+}: C_{m, \operatorname{tran}} \rightarrow C_{m, \operatorname{tran}}^{\prime}$ le foncteur qui à $\left(M, \phi_{M}\right)$ associe $\left(D, \phi_{D}, V_{D}, \mathcal{M}\right)$. Pour montrer que $\mathcal{M}$ est fortement divisible le calcul est le même qu'au paragraphe précédent en ajoutant des exposants $m$ et en utilisant le fait que ${ }^{\tau}\left(\& \cap(z-\pi)^{m} L[[z-\pi]]\right) \subset z^{m} \phi$, puisque $(z-\pi)^{-m} \& \cap L[[z-\pi]]$ est inclus dans le complété $z$-adique de $\phi+\frac{\pi^{q-m}}{z} \phi+\frac{\pi^{q^{2}-m}}{z^{q+1}} \phi+\cdots$ Donc le foncteur $\mathbb{D}^{+}$est bien défini.

ThÉORÈMe 11.9. - Le foncteur $\mathbb{D}^{+}: C_{m, \operatorname{tran}} \rightarrow C_{m \text {,tran }}^{\prime}$ est une équivalence de catégories. De plus pour tout $\left(D, \phi_{D}, V_{D}, \mathcal{M}\right) \in C_{m, \operatorname{tran}}^{\prime}$, l'image de $\mathcal{M}$ dans ${ }^{\tau} D \otimes_{k((z))} \phi\left[\frac{1}{z}\right]$ est stable

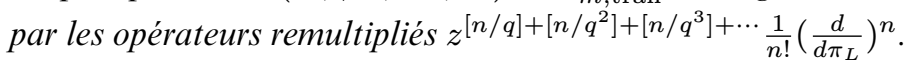

Il résulte du théorème que pour tout $\left(D, \phi_{D}, V_{D}, \mathcal{M}\right) \in C_{m, \operatorname{tran}}^{\prime},\left(D, \phi_{D}, V_{D}\right)$ est faiblement admissible.

Démonstration. - La démonstration est essentiellement la même que celle du théorème 10.3. Le fait que pour tout $\left(D, \phi_{D}, V_{D}, \mathcal{M}\right)$ dans l'image de $\mathbb{D}^{+}, \mathcal{M}$ est stable par les opérateurs remultipliés a été justifié après le lemme 11.4 (dont c'est une conséquence). Donc il reste à montrer que $\mathbb{D}^{+}$est une équivalence de catégories.

La pleine fidélité de $\mathbb{D}^{+}$résulte à nouveau de la proposition $4.1: \mathcal{M}$ détermine la réduction de $M$ modulo $\pi \theta_{L}$ et comme $\frac{\pi^{q-1}}{\pi^{m}}$ est nilpotent dans $B$ (grâce à l'hypothèse $m \leq q-2$ ), la proposition 4.1 implique que $\left(M, \phi_{M}\right)$ est déterminé par sa classe d'isogénie et par la réduction de $M$ modulo $\pi \theta_{L}$. Nous ne reprenons pas ici l'argument comme nous l'avions fait dans la démonstration du théorème 10.3.

Pour montrer que le foncteur est essentiellement surjectif, on construit $M$ à partir de $\mathcal{M}$ par le même procédé que dans le paragraphe précédent. Pour tout $a \in \mathbb{N}^{*}$, on note $\phi^{a}$ le complété $z$-adique de $\theta \widehat{\otimes} \vartheta_{L}\left[\frac{\pi^{a}}{z}, \frac{\pi^{a q}}{z^{q+1}}, \frac{\pi^{a q^{2}}}{z^{q^{2}+q+1}}, \ldots\right]$ de sorte que $\phi=\phi^{q}$. On énonce d'abord une variante du lemme 10.4.

Lemme 11.10. - a) Soient $a, m \in \mathbb{N}^{*}, a \geq m+2, \mathcal{N}$ un $\phi^{a}$-module libre et $W$ un sous- $L[[z-\pi]]-$ module de $\mathcal{N} \otimes_{\phi^{a}} L[[z-\pi]]$ contenant $(z-\pi)^{m} \mathcal{N} \otimes_{\phi^{a}} L[[z-\pi]]$. Alors $\mathcal{N}^{\prime}=\phi^{a-m} \cdot(\mathcal{N} \cap W)$ est un sous- $\phi^{a-m}$-module libre de $\mathcal{N} \otimes_{\phi^{a}} \phi^{a-m}$,

$$
\mathcal{N}^{\prime} \otimes_{\phi^{a-m}} \phi^{a-m}\left[\frac{1}{z-\pi}\right]=\mathcal{N} \otimes_{\phi^{a}} \phi^{a-m}\left[\frac{1}{z-\pi}\right] \text { et } \mathcal{N}^{\prime} \otimes_{\phi^{a-m}} L[[z-\pi]]=W .
$$

De plus $\operatorname{det}\left(\mathcal{N}^{\prime}\right)=(z-\pi)^{k} \operatorname{det}(\mathcal{N}) \otimes_{\phi^{a}} \phi^{a-m}$ où $k=\operatorname{dim}_{L}\left(\mathcal{N} \otimes_{\phi^{a}} L[[z-\pi]] / W\right)$.

b) Soit de plus $N$ un $\Theta \widehat{\otimes} \theta_{L}$-module libre muni d'un isomorphisme

$$
N \otimes_{\vartheta \widehat{\otimes} \vartheta_{L}} \phi^{a}=\mathcal{N} \text {. }
$$


Alors l'inclusion $\phi^{a-m} \cdot(N \cap W) \subset \phi^{a-m} \cdot(\mathcal{N} \cap W)$ est une égalité.

c) Soient de plus $b \geq$ a et $\widetilde{\mathcal{N}}$ un $\phi^{b}$-module libre muni d'un isomorphisme $\widetilde{\mathcal{N}} \otimes_{\phi^{b}} \phi^{a}=\mathcal{N}$. Alors l'inclusion $\phi^{a-m} \cdot(\widetilde{\mathcal{N}} \cap W) \subset \phi^{a-m} \cdot(\mathcal{N} \cap W)$ est une égalité.

Démonstration. - On montre simultanément a) et b). Soit $N$ un $\theta \widehat{\otimes} \widehat{\theta}_{L}$-module libre muni d'un isomorphisme $N \otimes_{\vartheta_{\widehat{\otimes} \vartheta_{L}}} \phi^{a}=\mathcal{N}$. D'après le lemme $8.1, N^{\prime}=N \cap W$ est un $\theta \widehat{\otimes} \theta_{L}$-module libre, et on a

$$
(z-\pi)^{m} N \subset N^{\prime} \subset N, \operatorname{det}\left(N^{\prime}\right)=(z-\pi)^{k} \operatorname{det}(N) \text { et } W=N^{\prime} \otimes_{\vartheta_{\widehat{\otimes} \vartheta_{L}}} L[[z-\pi]] .
$$

Comme $\mathcal{N}=N \otimes_{\vartheta \widehat{\otimes} \vartheta_{L}} \phi^{a}$ il résulte de ce qui précède que

$$
\mathcal{N} \cap W \subset N^{\prime} \otimes_{\vartheta \widehat{\otimes} \vartheta_{L}}\left((z-\pi)^{-m} \oint^{a} \cap L[[z-\pi]]\right) .
$$

On vérifie facilement que $(z-\pi)^{-m} \phi^{a} \cap L[[z-\pi]]$ est inclus dans le complété $z$-adique de $\phi^{a}+\frac{\pi^{a-m}}{z} \phi^{a}+\frac{\pi^{a q-m}}{z^{q+1}} \phi^{a}+\cdots$ qui est lui-même inclus dans $\phi^{a-m}$. Donc

$$
\mathcal{N}^{\prime} \subset N^{\prime} \otimes_{\partial \widehat{\otimes} \theta_{L}} \phi^{a-m}
$$

et on a l'égalité puisque $\mathcal{N} \cap W$ contient évidemment $N^{\prime}$. De plus

$$
\operatorname{det}\left(\mathcal{N}^{\prime}\right)=\operatorname{det}\left(N^{\prime}\right) \otimes_{\vartheta \widehat{\otimes} \vartheta_{L}} \phi^{a-m} .
$$

Enfin c) découle de b).

Suite de la démonstration du théorème 11.9. - Montrons maintenant que le foncteur $\mathbb{D}^{+}: C_{m, \operatorname{tran}} \rightarrow C_{m, \text { tran }}^{\prime}$ est essentiellement surjectif. Soit $\left(D, \phi_{D}, V_{D}, \mathcal{M}\right)$ un objet de $C_{m, \text { tran }}^{\prime}$. D'après le a) du lemme 11.10,

$$
\phi^{q-m} \cdot\left(\mathcal{M} \cap(z-\pi)^{m} V_{D}\right)
$$

est un $\phi^{q-m}$-module libre. On note $M_{0}$ son image $\operatorname{par}(z-\pi)^{-m} \phi_{D} \otimes 1$, si bien que

$$
M_{0}=\phi^{q-m} \cdot(z-\pi)^{-m}\left(\phi_{D} \otimes 1\right)\left(\mathcal{M} \cap(z-\pi)^{m} V_{D}\right)
$$

est un $\phi^{q-m}$-module libre naturellement inclus dans

$$
(z-\pi)^{-m} D \otimes_{k((z))} \phi^{q-m}\left[\frac{1}{z}\right] .
$$

Alors ${ }^{\tau} M_{0}$ est un $\phi^{q(q-m)}$-module libre qui est naturellement inclus dans

$$
\left(z-\pi^{q}\right)^{-m \tau} D \otimes_{k((z))} 8^{q(q-m)}\left[\frac{1}{z}\right] .
$$

Par l'hypothèse de forte divisibilité de $\mathcal{M}$, et comme $1-\frac{\pi^{q}}{z}$ est une unité dans $\phi$, on a ${ }^{\tau} M_{0} \otimes_{\phi q(q-m)} \&=\mathcal{M}$.

On définit par récurrence $M_{n}$ pour $n \in \mathbb{N}$ en posant

$$
M_{n+1}=8^{m_{n+1}} \cdot(z-\pi)^{-m}\left(\phi_{D} \otimes 1\right)\left({ }^{\tau} M_{n} \cap(z-\pi)^{m} V_{D}\right),
$$

où la suite $m_{n}$ est définie par $m_{0}=q-m$ et $m_{n+1}=q m_{n}-m$ (cette suite est strictement croissante et tend vers l'infini). Par le a) du lemme 11.10, $M_{n}$ est un $8^{m_{n}}$-module libre, inclus dans $(z-\pi)^{-m} \cdots\left(z-\pi^{q^{n}}\right)^{-m} D \otimes_{k((z))} \phi^{m_{n}}\left[\frac{1}{z}\right]$. L'hypothèse de forte divisibilité de $\mathcal{M}$, et le c) du lemme 11.10 (appliqué à $a=q, b=q m_{n-1}, \mathcal{N}=\mathcal{N}$ et $\widetilde{\mathcal{N}}={ }^{\tau} M_{n-1}$ ) montrent, par récurrence sur $n,{ }^{\tau} M_{n} \otimes_{\phi q m_{n}} \phi=\mathcal{M}$.

On a déjà vu que ${ }^{\tau} M_{0} \subset \mathcal{M}$. On en déduit $M_{1} \subset M_{0}$. Cette assertion a un sens car les deux

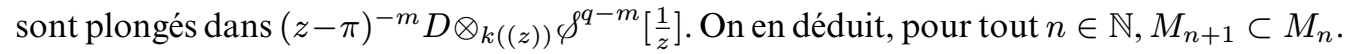


En fait l'égalité ${ }^{\tau} M_{0} \otimes_{\phi^{q m_{0}}} \phi=\mathcal{M}$ implique, grâce au c) du lemme 11.10 (appliqué à $a=q, b=q m_{0}, \mathcal{N}=\mathcal{M}$ et $\widetilde{\mathcal{N}}={ }^{\tau} M_{0}$ ), que l'inclusion $M_{1} \subset M_{0}$ induit une égalité $M_{1} \otimes_{\phi^{m_{1}}} \phi^{m_{0}}=M_{0}$. On montre alors, par récurrence sur $n$, grâce au c) du lemme 11.10 (appliqué à $a=q m_{n-1}, b=q m_{n}, \mathcal{N}={ }^{\tau} M_{n-1}$ et $\widetilde{\mathcal{N}}={ }^{\tau} M_{n}$ ), que l'inclusion $M_{n+1} \subset M_{n}$ induit une égalité $M_{n+1} \otimes_{\delta^{m_{n+1}}} \phi^{m_{n}}=M_{n}$. En particulier comme $m_{n+1}>m_{n}>q^{n}$, l'inclusion $M_{n+1} \subset M_{n}$ induit une égalité modulo $\pi^{q^{n}}$, c'est-à-dire

$$
M_{n+1} \otimes_{\phi^{m_{n+1}}} \oslash \widehat{\otimes} \theta_{L} / \pi^{q^{n}}=M_{n} \otimes_{\phi^{m_{n}}} \theta \widehat{\otimes} \theta_{L} / \pi^{q^{n}} .
$$

On prend alors $M$ égal à l'intersection des $M_{n}$.

On remarque que $Q_{n}=M_{n} \otimes \phi^{m_{n}} \theta \widehat{\otimes} \widehat{\otimes} \theta_{L} / \pi^{q^{n}}$ est un $\theta \widehat{\otimes} \theta_{L} / \pi^{q^{n}}$-module libre de rang

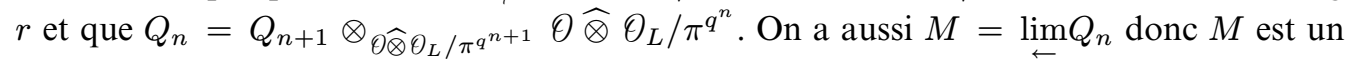
$\oslash \widehat{\otimes} \vartheta_{L}$-module libre de rang $r$. De plus pour tout $n \in \mathbb{N}$ l'inclusion $M \subset M_{n}$ induit une égalité $M \otimes_{\vartheta \widehat{\otimes} \vartheta_{L}} \phi^{m_{n}}=M_{n}$.

Comme $M_{n+1}=\phi^{m_{n+1}} \cdot(z-\pi)^{-m}\left(\phi_{D} \otimes 1\right)\left({ }^{\tau} M_{n} \cap(z-\pi)^{m} V_{D}\right)$ pour tout $n \in \mathbb{N}$, et grâce au b) du lemme 11.10, on a

$$
M=(z-\pi)^{-m}\left(\phi_{D} \otimes 1\right)\left({ }^{\tau} M \cap(z-\pi)^{m} V_{D}\right) .
$$

On définit $(z-\pi)^{m} \phi_{M}^{-1}: M \rightarrow{ }^{\tau} M$ comme la restriction de $(z-\pi)^{m}\left(\phi_{D} \otimes 1\right)^{-1}$ à $M$. On a alors $\phi_{M} \in \operatorname{Hom}_{\vartheta \widehat{\otimes} \vartheta_{L}}\left({ }^{\tau} M, M\right)$.

Comme $M \otimes_{\partial \widehat{\otimes} \vartheta_{L}} \phi^{m_{n}}=M_{n}$ et ${ }^{\tau} M_{n} \otimes_{\phi^{q m_{n}}} \phi=\mathcal{M}$ (pour n'importe quelle valeur de $n$ ) on a ${ }^{\tau} M \otimes_{\partial \widehat{\otimes} \vartheta_{L}} \&=M$.

Le théorème 11.9 est analogue à la conjecture 2.2.6 (1) dans [14] (limitée au cas cristallin), qui a été démontrée par Tong Liu (théorème 2.3.5 de [45]), en utilisant les résultats de Kisin [39], après des résultats partiels de Breuil et Caruso [13, 14, 15]. Les articles [13, 39, 45] utilisent la théorie du corps des normes [12,23]. Les $\mathbb{Z}_{p}$-réseaux stables par le groupe de Galois de $K\left(\pi^{p^{-\infty}}\right.$ ) (où $\pi$ est une uniformisante de $K$ extension finie de $\mathbb{Q}_{p}$ ) sont classifiés par des objets qui ressemblent beaucoup aux chtoucas locaux. On peut rapprocher notre condition de tranquillité de la condition de logarithmicité des pôles de la connexion canonique qui figure dans le corollaire 1.3.15 de [39].

Notre anneau $\&$ est exactement le $\theta$-analogue de l'anneau $\&$ de Breuil [14]. La condition de forte divisibilité y est exprimée sous une forme un peu différente mais équivalente. La filtration sur $\varnothing$ introduite par Breuil a pour analogue la filtration $\& \cap(z-\pi)^{i} L[[z-\pi]]$. L'opérateur $N$ de Breuil est $-u \frac{d}{d u}$ et correspond à $-\pi_{L} \frac{d}{d \pi_{L}}$. Cet opérateur suffit chez Breuil, alors que pour exprimer la condition de tranquillité de la structure de Hodge-Pink nous avons besoin des opérateurs remultipliés, parce qu'en inégales caractéristiques $\left(-u \frac{d}{d u}\right)^{p}$ est non nul, alors qu'il est nul en égales caractéristiques (pour la condition de tranquillité de la structure de Hodge-Pink le coefficient devant l'opérateur différentiel n'a pas d'importance ... du moment qu'il est non nul). On notera que la condition de stabilité de $\mathcal{M}$ par $u \frac{d}{d u}$ qui figure dans $[14,45]$ n'apparaît pas ici dans la définition de $C_{m, \operatorname{tran}}^{\prime}$.

Le théorème 11.9 affirme que l'image de $\mathcal{M}$ dans ${ }^{\tau} D \otimes_{k((z))} \&\left[\frac{1}{z}\right]$ est stable par les opérateurs remultipliés $z^{[n / q]+\left[n / q^{2}\right]+\left[n / q^{3}\right]+\cdots} \frac{1}{n !}\left(\frac{d}{d \pi_{L}}\right)^{n}$. Pour comparer ces opérateurs à ceux 
de [14], les coordonnées $(u, \pi)$ de $\phi_{\min }$ venant de l'isomorphisme $\phi_{\min }=\hat{B}$ de la proposition 10.2 sont préférables. On verra donc $\&$ comme le complété pour la topologie $\pi$-adique de $(\Theta \widehat{\otimes} k)[[u]]\left[\frac{u^{e q}}{\pi}, \frac{u^{e q^{2}}}{\pi^{q+1}}, \ldots\right]$. Donc le théorème 11.9 affirme que $\mathcal{M}$ est stable par les opé-

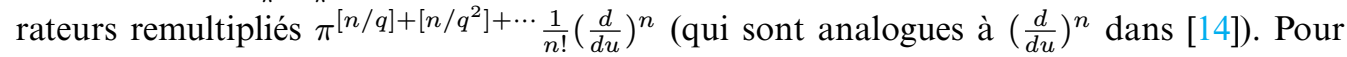
nous ces opérateurs agissent trivialement $\operatorname{sur}^{\tau} D$ puisque nous étudions ici le cas de bonne réduction i.e. $N=0$ chez Breuil. L'opérateur $u \frac{d}{d u}$ apparaît à la place de $\frac{d}{d u}$ dans [14] parce que Breuil traite en même temps le cas semi-stable (on notera que dans le théorème 3.2.5 de [14], qui ne concerne que le cas cristallin, $\mathcal{M}$ est stable par $\frac{d}{d u}$ ).

En suivant l'exemple 2.2.2 (2) de [14], nous remarquons enfin que lorsque $e=1$, le théorème 11.9 donne un analogue en égales caractéristiques de la théorie de FontaineLaffaille [24]. En effet il résulte de la deuxième remarque après le lemme 11.8 que pour $\left(D, \phi_{D}, V_{D}, \mathcal{M}\right)$ dans l'image essentielle de $\mathbb{D}^{+}: C_{m, \operatorname{tran}} \rightarrow C_{m, \operatorname{tran}}^{\prime}$, on a $\mathcal{M}={ }^{\tau} \Delta \otimes_{k[[z]]} \phi$. De plus on vérifie facilement, en utilisant la tranquillité de $V_{D}$, que la forte divisibilité de ${ }^{\tau} \Delta \otimes_{k[[z]]} \&$ équivaut à la condition de Fontaine-Laffaille, c'est-à-dire, en notant ${ }^{\tau} D=\mathrm{Fil}^{0} \supset \mathrm{Fil}^{1} \supset \cdots \supset \mathrm{Fil}^{m}=\{0\}$ la suite de $L=k((z))$-espaces vectoriels définie par (79), $\phi_{D}\left(\sum_{i=0}^{m-1} z^{-i}\left({ }^{\tau} \Delta \cap \mathrm{Fil}^{i}\right)\right)=\Delta$.

\section{RÉFÉRENCES}

[1] V. Abrashkin, Galois modules arising from Faltings's strict modules, Compos. Math. 142 (2006), 867-888.

[2] G. Anderson, Drinfeld motives, cours donné à Princeton du 27 octobre au 8 décembre 1987.

[3] L. Berger, Équations différentielles $p$-adiques et $(\phi, N)$-modules filtrés, Astérisque 319 (2008), 13-38.

[4] P. Berthelot, Cohomologie cristalline des schémas de caractéristique $p>0$, Lect. Notes in Math. 407 (1974).

[5] P. Berthelot, Cohomologie rigide et cohomologie rigide à supports propre, prépublication 96-03 de l'université de Rennes, http: //perso . univ-rennes1. fr/pierre. berthelot/publis/Cohomologie_Rigide_I.pdf, 1996.

[6] P. Berthelot, L. Breen, W. Messing, Théorie de Dieudonné cristalline. II, Lect. Notes in Math. 930 (1982).

[7] P. Berthelot, W. Messing, Théorie de Dieudonné cristalline. I, in Journées de Géométrie Algébrique de Rennes (Rennes, 1978), Vol. I, Astérisque 63, Soc. Math. France, 1979, 17-37.

[8] P. Berthelot, W. Messing, Théorie de Dieudonné cristalline. III. Théorèmes d'équivalence et de pleine fidélité, in The Grothendieck Festschrift, Vol. I, Progr. Math. 86, Birkhäuser, 1990, 173-247.

[9] P. Berthelot, A. Ogus, Notes on crystalline cohomology, Princeton Univ. Press, 1978. 
[10] S. Bosch, U. Güntzer, R. Remmert, Non-Archimedean analysis, Grund. Math. Wiss. 261, Springer, 1984.

[11] N. Bourbaki, Algèbre commutative. Chapitres 8 et 9, Masson, 1983.

[12] C. BReuil, Schémas en groupes et corps des normes, prépublication http://www. ihes.fr/ breuil/PUBLICATIONS/groupesnormes.pdf, 1998.

[13] C. Breuil, Une application de corps des normes, Compositio Math. 117 (1999), 189203.

[14] C. Breuil, Integral p-adic Hodge theory, in Algebraic geometry 2000, Azumino (Hotaka), Adv. Stud. Pure Math. 36, Math. Soc. Japan, 2002, 51-80.

[15] X. Caruso, Représentations semi-stables de torsion dans le case $e r<p-1$, J. reine angew. Math. 594 (2006), 35-92.

[16] P. Colmez, J.-M. Fontaine, Construction des représentations $p$-adiques semi-stables, Invent. Math. 140 (2000), 1-43.

[17] V. G. DrinfeL'D, Coverings of $p$-adic symmetric domains, Funkcional. Anal. i Priložen. 10 (1976), 29-40; traduction : Funct. Anal. Appl. 10 (1976), 107-115.

[18] V. G. Drinfel'D, Cohomology of compactified moduli varieties of $F$-sheaves of rank 2, Zap. Nauchn. Sem. Leningrad. Otdel. Mat. Inst. Steklov. (LOMI) 162 (1987), 107-158; traduction : J. Soviet Math. 46 (1989), 1789-1821.

[19] V. G. Drinfel'D, Moduli varieties of F-sheaves, Funktsional. Anal. i Prilozhen. 21 (1987), 23-41; traduction : Funct. Anal. Appl. 21 (1987), 107-122.

[20] G. Faltings, Integral crystalline cohomology over very ramified valuation rings, J. Amer. Math. Soc. 12 (1999), 117-144.

[21] G. Faltings, Group schemes with strict $\mathscr{O}$-action, Mosc. Math. J. 2 (2002), 249-279.

[22] J.-M. Fontaine, Groupes p-divisibles sur les corps locaux, Astérisque 47-48 (1977).

[23] J.-M. Fontaine, Représentations $p$-adiques des corps locaux. I, in The Grothendieck Festschrift, Vol. II, Progr. Math. 87, Birkhäuser, 1990, 249-309.

[24] J.-M. Fontaine, G. Laffaille, Construction de représentations p-adiques, Ann. Sci. École Norm. Sup. 15 (1982), 547-608.

[25] F. GARDEYN, The structure of analytic $\tau$-sheaves, J. Number Theory 100 (2003), 332362.

[26] A. Genestier, Espaces symétriques de Drinfeld, Astérisque 234 (1996).

[27] A. Grothendieck, Crystals and the de Rham cohomology of schemes, in Dix Exposés sur la Cohomologie des Schémas, North-Holland, 1968, 306-358.

[28] A. Grothendieck, Groupes de Barsotti-Tate et cristaux, in Actes du Congrès International des Mathématiciens (Nice, 1970), Tome 1, Gauthier-Villars, 1971, 431-436.

[29] U. Hartl, On period spaces for p-divisible groups, C. R. Math. Acad. Sci. Paris 346 (2008), 1123-1128.

[30] U. Hartl, A dictionary between Fontaine-theory and its analogue in equal characteristic, J. Number Theory 129 (2009), 1734-1757.

[31] U. HartL, Period spaces in equal characteristic, prépublication arXiv :math/0511686, to appear in Annals of Math. .

[32] U. Hartl, R. Pink, Vector bundles with a Frobenius structure on the punctured unit disc, Compos. Math. 140 (2004), 689-716.

$4^{\mathrm{e}}$ SÉRIE - TOME $44-2011$ - No 2 
[33] M. J. Hopkins, B. H. Gross, Equivariant vector bundles on the Lubin-Tate moduli space, in Topology and representation theory (Evanston, IL, 1992), Contemp. Math. 158, Amer. Math. Soc., 1994, 23-88.

[34] L. ILlusie, Déformations de groupes de Barsotti-Tate (d'après A. Grothendieck), Astérisque 127 (1985), 151-198.

[35] M. Kapranov, É. Vasserot, Formal loops. II. A local Riemann-Roch theorem for determinantal gerbes, Ann. Sci. École Norm. Sup. 40 (2007), 113-133.

[36] N. M. Katz, Slope filtration of F-crystals, in Journées de Géométrie Algébrique de Rennes (Rennes, 1978), Vol. I, Astérisque 63, Soc. Math. France, 1979, 113-163.

[37] N. M. Katz, Serre-Tate local moduli, Lect. Notes in Math. 868 (1981), 138-202.

[38] K. S. Kedlaya, Slope filtrations revisited, Doc. Math. 10 (2005), 447-525.

[39] M. Kisin, Crystalline representations and F-crystals, in Algebraic geometry and number theory, Progr. Math. 253, Birkhäuser, 2006, 459-496.

[40] M. Kisin, Moduli of finite flat group schemes, and modularity, Ann. of Math. 170 (2009), 1085-1180.

[41] G. Laffaille, Groupes p-divisibles et modules filtrés : le cas peu ramifié, Bull. Soc. Math. France 108 (1980), 187-206.

[42] L. Lafforgue, Une compactification des champs classifiant les chtoucas de Drinfeld, J. Amer. Math. Soc. 11 (1998), 1001-1036.

[43] L. Lafforgue, Cours à l'Institut Tata sur les chtoucas de Drinfeld et la correspondance de Langlands, prépublication IHÉS M/02/45 http://www.ihes.fr/ Iafforgue/math/M02-45.pdf.

[44] S. G. LAngton, Valuative criteria for families of vector bundles on algebraic varieties, Ann. of Math. 101 (1975), 88-110.

[45] T. LiU, On lattices in semi-stable representations : a proof of a conjecture of Breuil, Compos. Math. 144 (2008), 61-88.

[46] H. Matsumura, Commutative ring theory, Cambridge Studies in Advanced Math. 8, Cambridge Univ. Press, 1986.

[47] B. Mazur, W. Messing, Universal extensions and one dimensional crystalline cohomology, Lect. Notes in Math. 370 (1974).

[48] W. Messing, The crystals associated to Barsotti-Tate groups, Lect. Notes in Math. 264 (1972).

[49] R. PINK, Hodge structures over function fields, prépublication http://www. math. ethz.ch/ pink/ftp/HS.pdf, 1997.

[50] R. Pink, Uniformisierung von $t$-Motiven, exposé du 12 juillet 2000.

[51] M. Rapoport, T. ZInk, Period spaces for p-divisible groups, Annals of Math. Studies 141, Princeton Univ. Press, 1996.

[52] M. RAPOPORT, T. ZINK, A finiteness theorem in the Bruhat-Tits building : an application of Landvogt's embedding theorem, Indag. Math. (N.S.) 10 (1999), 449-458.

[53] J-P. Serre, Corps locaux, 2e éd., Publications de l'Université de Nancago VIII, Hermann, 1968.

[54] J-P. Serre, Classes des corps cyclotomiques (d'après K. Iwasawa), Sém. Bourbaki 1958/59, exp. nº 174, réédition Soc. Math. France 5 (1995), 83-93. 
[55] T. ZINK, Windows for displays of $p$-divisible groups, in Moduli of abelian varieties (Texel Island, 1999), Progr. Math. 195, Birkhäuser, 2001, 491-518.

(Manuscrit reçu le 15 décembre 2008; accepté, après révision, le 25 mai 2010.)

\author{
Alain Genestier \\ Institut Élie Cartan \\ Université Henri Poincaré Nancy 1 \\ B.P. 70239 \\ 54506 Vandœuvre-lès-Nancy, France \\ E-mail: Alain.Genestier@iecn.u-nancy.fr \\ Vincent LAFFORGUE \\ CNRS et Institut de mathématiques de Jussieu \\ 175 rue du Chevaleret \\ 75013 Paris, France \\ E-mail: vlafforg@math.jussieu.fr
}

